\title{
몬
}

Universidad Autónoma Metropolitana - Iztapalapa

División de Ciencias Básicas e Ingenicría

Departamento de Ingeniería de Procesos e Hidráulica

Titulo:

\section{ESTIMACIÓN Y CONTROL NO LINEAL DE REACTORES CONTINUOS DE COPOLIMERIZACIÓN EN SOLUCIÓN}

\author{
Tesis que presenta \\ M.I.Q. María Teresa López Arenas \\ Matricula: 95242176 \\ Para obtener el grado de : \\ Doctor en Ciencias
}

Asesor:

Dr. Jesús Alvarez Calderón

Octubre del 2000 


\section{Agradecimientos}

Extiendo mi agradecimiento:

Al Dr. Jesús Alvarez Calderón por su asesoria y apoyo invaluable para desarrollar y concluir esta tesis.

A la Universidad Autónoma Metropolitana-Iztapalapa, y en particular al Area de Ingeniería Química, por el favorable ambiente y formación del posgrado.

Al grupo de Control de Procesos (estudiantes de la UAM-I y colegas del Grupo Comex) por innumerables discusiones e intercambio de ideas enriquecedoras.

Al CONACyT por el apoyo económico otorgado (registro 92549) para realizar estudios de posgrado.

Al Centro de Investigación en Polímeros (Grupo Comex) por el apoyo económico (Galardón otorgado a estudiantes de posgrado), asi como por las facilidades otorgadas para la realización de esta tesis.

Un especial agradecimiento:

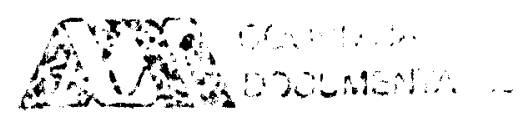

A Mauricio Sales, Martha Carmona, y René Nakamura por su ánimo y apoyo para concluir este trabajo.

A mis papás Tere y Paco, y a mis hermanos Male, Maru, Rosy, Paco, Maguis, Fer y Chay por su compresión y su apoyo incondicional; $y$ a quienes les dedico esta tesis. 


\section{Resumen}

Muchos plásticos comerciales y de ingeniería son industrialmente producidos en reactores de copolimerización en solución. El propósito fundamental de la copolimerización, a escala comercial, es mejorar las propiedades fisicas, quimicas, y mecánicas de un homopolímero. Debido a esto, el conocimiento de las propiedades instantáneas del copolimero (tales como: la conversión, la composición de copolímero, los pesos moleculares, la fracción de sólidos, etc.) durante su proceso de producción es importante para monitorear y controlar los indices de desempeño relacionados a la seguridad, la operabilidad, la productividad, y el grado o calidad del producto.

En esta tesis se presenta una metodologia para abordar los problemas de estimación y de control en reactores continuos de copolimerización en solución. E1 objetivo principal es el diseño de estimadores y controladores (geométricos) no lineales que permitan inferir propiedades no medibles en tiempo real (como son la fracción de sólidos, la composición instantánea, la conversión, el peso molecular peso-promedio, y la velocidad de producción del copolimero), empleando un modelo dinámico del proceso en combinación con una retroalimentación de salidas medibles en línea (seleccionando mediciones continuas, instantáneas y de bajo costo, como son densidad, indice de refracción, temperatura, y volumen).

La metodología propuesta aborda tres aspectos principalmente. Primero, el estudio de los reactores continuos de copolimerización en solución. Donde la finalidad es describir, entender, caracterizar e interpretar el comportamiento dinámico del reactor mediante un sistema de ecuaciones diferenciales no lineales. Segundo, el esquema de estimación no lineal (a lazo abierto). En el cual se desarrolla un estimador geométrico no lineal, con las siguientes caracteristicas: (a) construcción sistemática, (b) diseño con criterio de convergencia, (c) técnica de sintonización simple y sistemática (mediante la asignación de polos, de forma similar a las técnicas de control y filtrado lineal convencional), y (d) la estructura de estimabilidad (i.e., grado de observabilidad y partición de los estados observables - no observables) como grado de libertad del diseño. Y tercero, el esquema de control no lineal con retroalimentación de mediciones. $\mathrm{El}$ cual se construye a partir de la combinación del estimador de estados a lazo abierto, con el control no lineal con retroalimentación de estados; mostrando características similares al estimador (en cuanto a diseño, construcción, sintonización, y convergencia).

Los esquemas de estimación y control propuestos son implementados para la copolimerización de metil metacrilato - acetato de vinilo, con acetato de etilo como solvente, y AIBN como iniciador. Los resultados muestran la factibilidad de inferir y regular las variables de calidad, seguridad, y velocidad de producción a partir de mediciones secundarias en linea. Tanto el estimador como el controlador no lineal muestran robustez ante errores en las condiciones iniciales, cambios en las entradas exógenas, errores en los parámetros del modelo, y ruido en las mediciones. 


\section{INDICE}

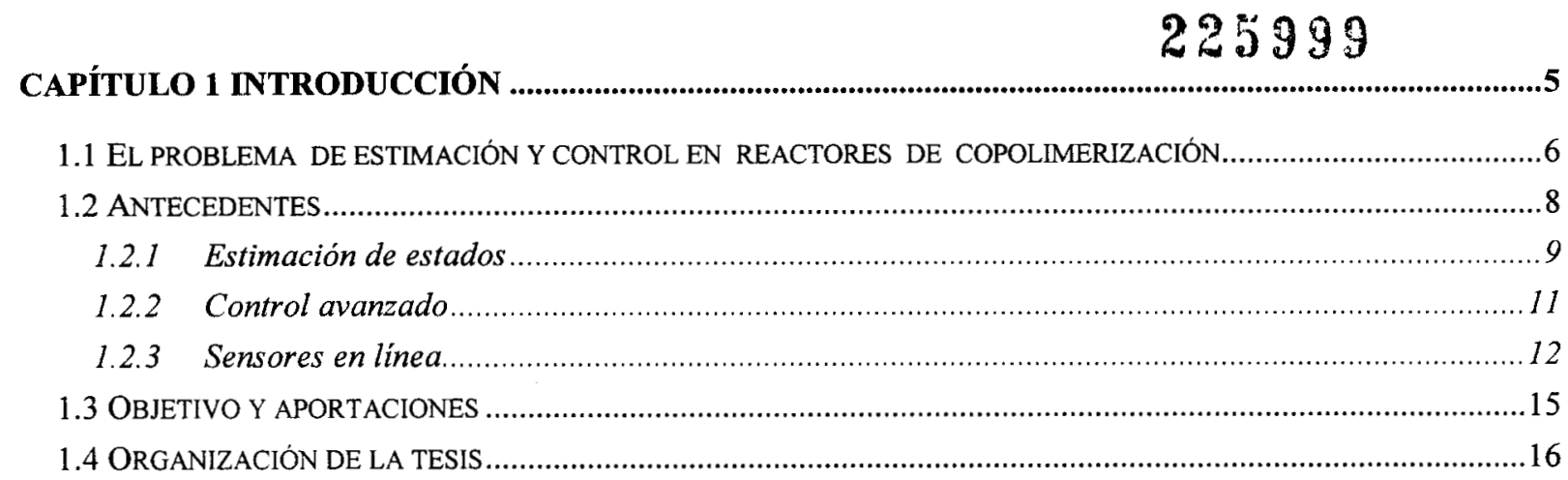

CAPÍTULO 2 REACTORES DE COPOLIMERIZACIÓN EN SOLUCIÓN

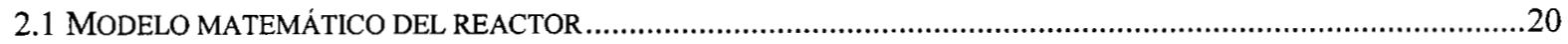

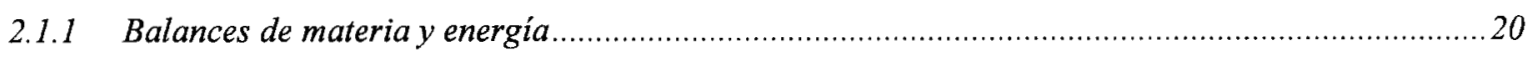

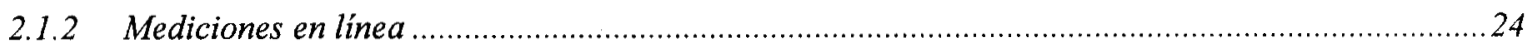

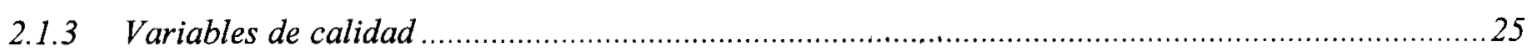

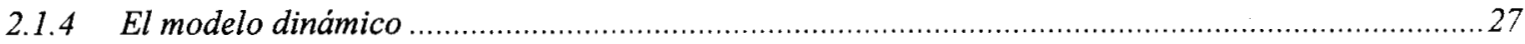

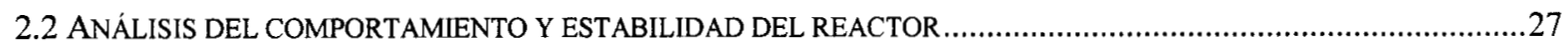

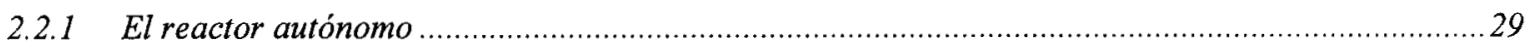

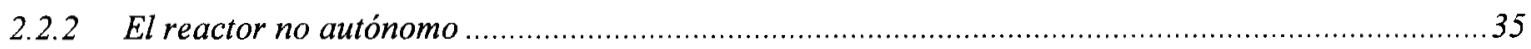

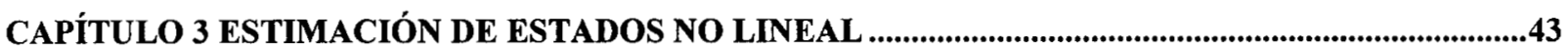

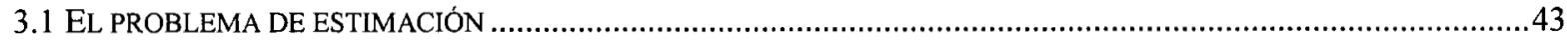

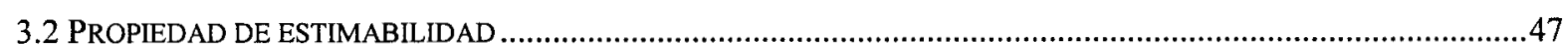

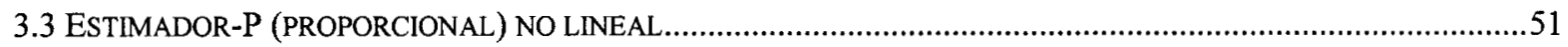

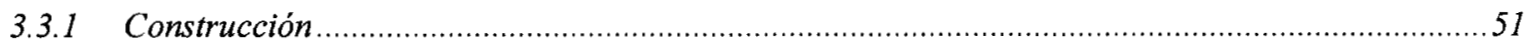

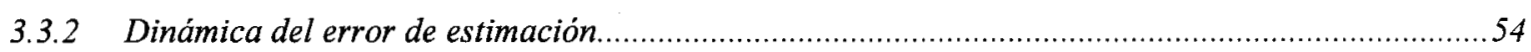

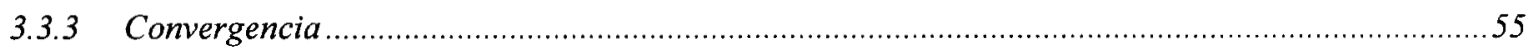

3.4 ESTIMADOR-PI (PROPORCIONAL INTEGRAL) NO LINEAL .........................................................................58

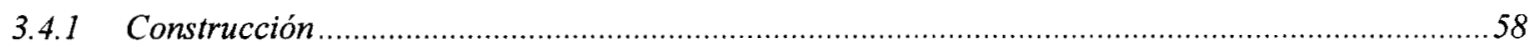

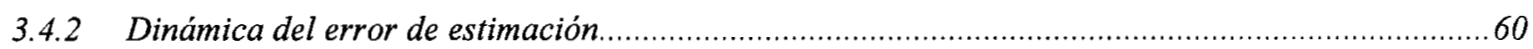

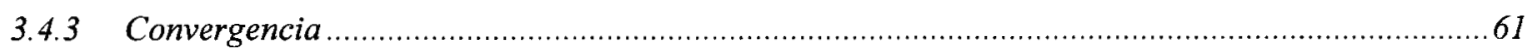

3.5 RESUMEN DEL DISEÑO DEL ESTIMADOR Y SUS GRADOS DE LIBERTAD...................................................63

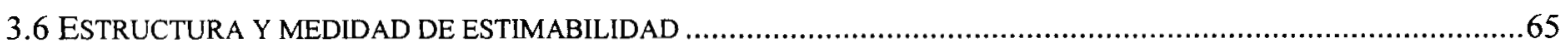

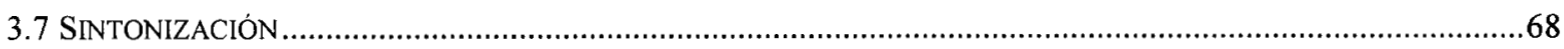




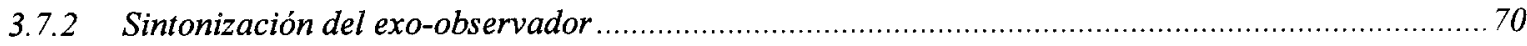

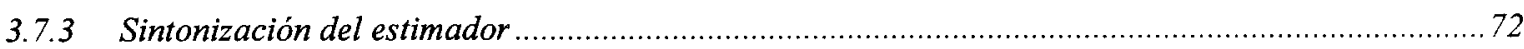

3.8 CONSIDERACIONES PARA LA IMPLEMENTACIÓN DE UN ESTIMADOR .........................................................74

CAPÍTULO 4 ESTIMACIÓN DE ESTADOS EN REACTORES DE COPOLIMERIZACIÓN ......................77

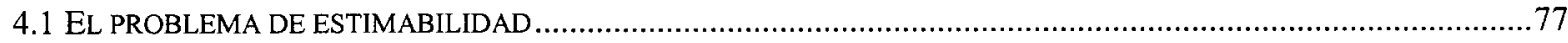

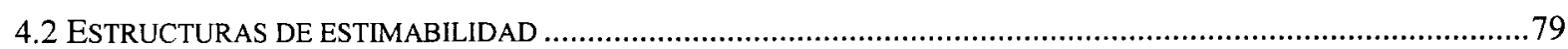

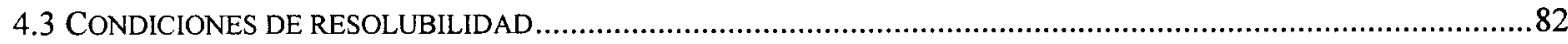

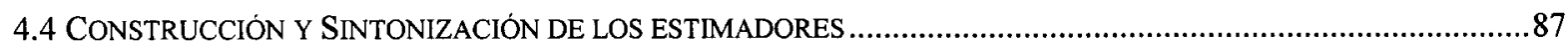

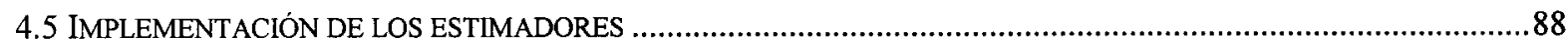

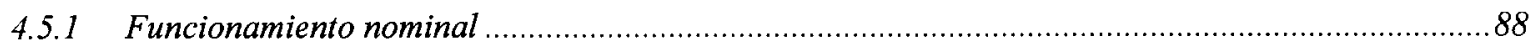

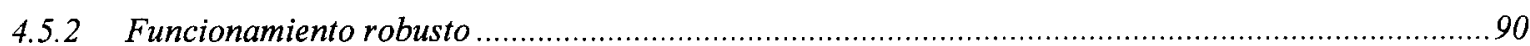

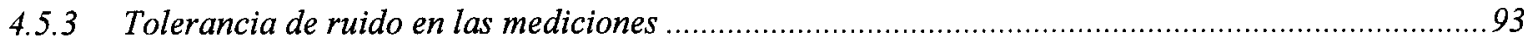

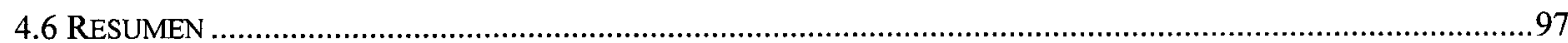

CAPÍTULO 5 CONTROL NO LINEAL CON RETROALIMENTACIÓN DE MEDICIONES.....................99

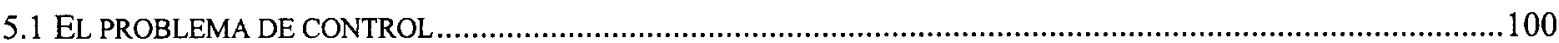

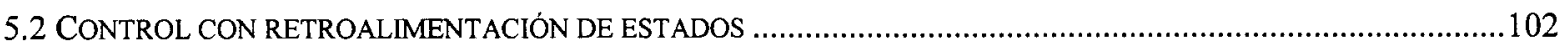

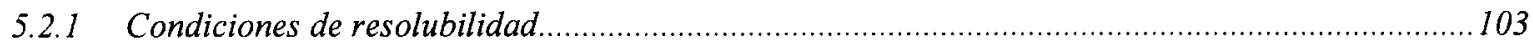

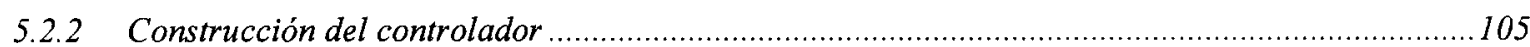

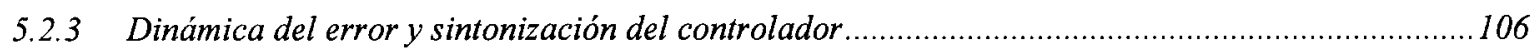

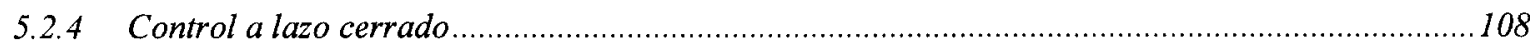

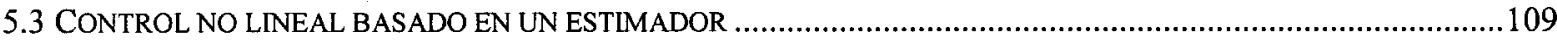

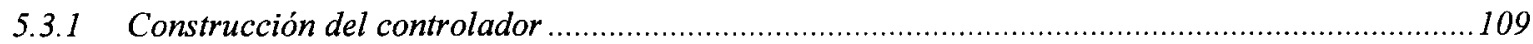

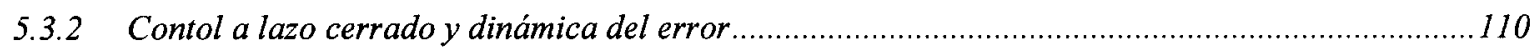

5.4 CONTROL DE REACTORES CONTINUNOS DE COPOLIMERIZACIÓN EN SOLUCIÓN.............................................112

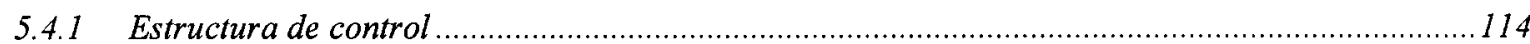

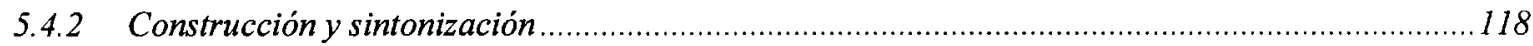

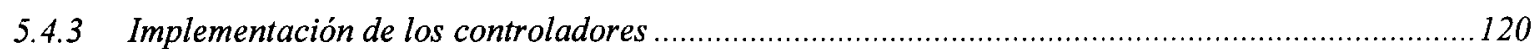

5.5 ALTERNATICA AL ESQUEMA DE CONTROL CON RETROALIMENTACION DE MEDICIONES................................126

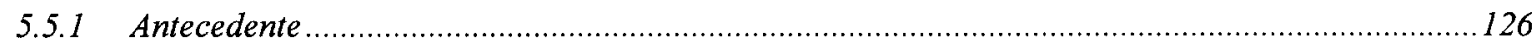

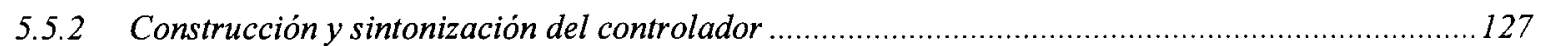

5.5.3 Implementación del controlador para el reactor continuo de copolimerización en solución...........130

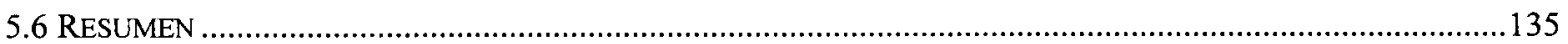

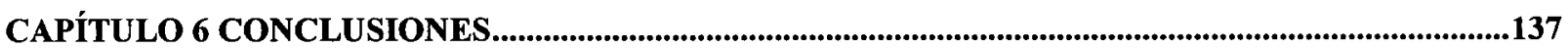




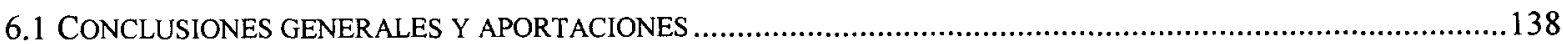

6.2 OTRAS APLICACIONES Y RECOMENDACIONES PARA TRABAJO FUTURO …..............................................140

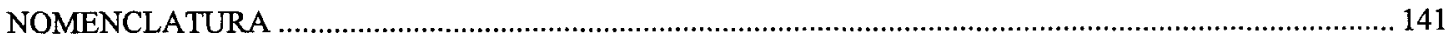

APÉNDICE A. Funcionalidades del modelo del reactor de copolimerización en solución.................................... 145

APÉNDICE B. Parámetros para el reactor de copolimerización de MMA-AV ................................................ 149

APÉNDICE C. Herramientas de sistemas dinámicos no lineales.................................................................... 151

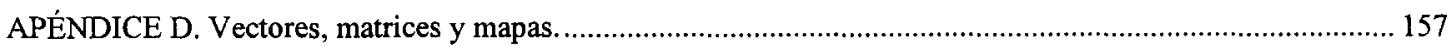

APÉNDICE E. Prueba de los Teoremas y Corolarios.................................................................................. 159

APÉNDICE F. Técnicas de Sintonización. .............................................................................................. 173

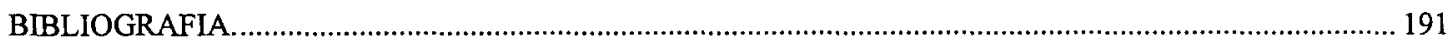


Indice

\section{4}




\section{Capítulo 1}

\section{INTRODUCCIÓN}

En este capítulo se plantea el problema de estimación y de control en los reactores de copolimerización, con un enfoque orientado hacia situaciones industriales. Primero se presenta una reseña bibliográfica que permite justificar y motivar el alcance de este trabajo de investigación. Posteriormente, se definen los objetivos principales, y se resume tanto la metodología como los aportes de la tesis. Por último, se describe la manera en que está organizada la tesis. 


\subsection{El problema de estim ación y control en reactores de copolimerización}

Muchos plásticos comerciales y de ingenieria son industrialmente producidos en reactores de copolimerización en solución. El propósito fundamental de la copolimerización, a escala comercial, es mejorar las propiedades físicas, químicas, y mecánicas de un homopolímero (como son: la resistencia química, la adhesión, la resistencia a la corrosión, la retención de color, etc.). Mediante la adición de pequeñas cantidades de un segundo monómero, es posible regular la velocidad de copolimerización, y consecuentemente la composición y propiedades del copolímero. Debido a esto, el conocimiento de las propiedades instantáneas del copolimero (tales como: la conversión, la composición de copolimero, los pesos moleculares, la fracción de sólidos, etc.) durante su proceso de producción es importante para monitorear y controlar los indices de desempeño relacionados a la seguridad, la operabilidad, la productividad, y el grado o calidad del producto.

Es un hecho conocido en la práctica industrial que, el monitoreo y la regulación de la composición instantánea del copolímero (esto es, la razón relativa de la incorporación de los dos comonómeros dentro del material polimérico) son un requisito clave para garantizar, en buena medida, algunos de los principales atributos de calidad del producto (Van Doremaele, 1990), por ejemplo: las propiedades de la distribución de cadena del copolímero, la magnitud y la unimodalidad de la distribución de temperatura de transición vítrea; las cuales a su vez determinan la homogeneidad termodinámica del material, su degradación y resistencia, y sus propiedades mecánicas. En la ingenieria industrial de reactores de polimerización, la eficiencia de manufactura de los polimeros funcionalizados (esto es, un homopolimero comercial barato copolimerizado con una pequeña cantidad de un comonómero de alto costo) representa un reto, especialmente cuando las especificaciones de calidad del producto son estrictas y los comonómeros tienen reactividades significativamente diferentes. En consecuencia, la posibilidad de tener estimados confiables (y en tiempo real) de, al menos, la composición instantánea del copolímero es un tema importante de investigación, con implicaciones que pueden tener efecto en la forma de diseñar, operar, y controlar los reactores de copolimerización.

Sin embargo, un problema serio para la implementación de estrategias de monitoreo y control es la disponibilidad de mediciones en linea de las propiedades de calidad del polimero. Las principales limitaciones son (Baratti et al., 1993; Dimitratos et al., 1991): 
(a) Los tiempos largos que en el laboratorio de control de calidad se requieren para analizar y reportar alguna propiedad, implicando esto una acción correctiva con retardo.

(b) El alto costo en inversión y mantenimiento del instrumento de medición.

(c) La escasez de instrumentos o sensores sofisticados y confiables.

(d) Los errores de medición (incluyendo ruido del instrumento) derivados de la técnica de análisis.

(e) La pobre confiabilidad que se puede tener ante ambientes hostiles, etc.

Por lo anterior, el enfoque principal está en diseñar estimadores que permitan inferir propiedades no medibles en tiempo real (como son la composición instantánea del copolímero, la concentración de iniciador, la distribución de pesos moleculares, etc.) empleando un modelo dinámico del proceso en combinación con una retroalimentación de salidas medibles en línea (basándose preferiblemente en mediciones continuas, instantáneas y de bajo costo, como son temperatura, presión, densidad, etc.).

Como se mencionó antes, los problemas de operación y control de los reactores de copolimerización están relacionados estrechamente con la seguridad, la calidad y especificaciones del producto, la rentabilidad, y la velocidad de producción. A medida que se exige una mejor calidad del producto y una mayor velocidad de producción se requieren condiciones de operación más severas, y consecuentemente el problema de control (o simplemente de monitoreo) llega a ser más dificil. Esto es debido a que altas velocidades de producción, con altas conversiones, implican una operación con comportamiento típico no lineal (Ray, 1986): tendencia a la inestabilidad del estado estacionario en un reactor continuo o de la trayectoria en un reactor por lotes, fuerte acoplamiento entre las entradas y salidas del proceso, y alta sensibilidad de las propiedades de calidad ante perturbaciones en las condiciones de operación. En principio, el uso de técnicas (avanzadas) apropiadas de control y monitoreo ofrece la posibilidad de operar los reactores a altas demandas de calidad y producción, manteniendo niveles adecuados de seguridad y operabilidad del proceso.

Hay varios aspectos que deben considerarse en el desarrollo de técnicas de control: (i) la definición y monitoreo de la calidad del producto, (ii) la inferencia de las variables de calidad a partir de mediciones en línea, (iii) el diseño de un control multivariable con compensación de interacciones que garantice la estabilidad interna [esto es, evitando multiplicidad de entradas como se ha encontrado en los trabajos de Balakotaiah y Luss (1985), y Adebekun y Schork (1989)], y con técnicas de construcción y sintonización simples. En principio, un mejor controlador debe obtenerse a partir de la construcción de un control con retroalimentación de salidas en combinación con un estimador de estados. No obstante, sin importar de que tipo 
de controlador se trate [ya sea control convencional lineal, control lineal multivariable con retroalimentación de salidas como en Congalidis et al. (1989), control no lineal con retroalimentación de estados como en Padilla et al. (1995), o bien control no lineal con retroalimentación de salidas como en Adebekun y Schork (1989) y en Alvarez (1996)], la inferencia de los estados y de las variables de calidad es un problema importante en la ingenieria de reactores de polimerización, que tiene fuertes implicaciones (además del monitoreo y control) en:

(a) La detección de fallas.

(b) El escalamiento.

(c) El diseño de estrategias de operación.

(d) La optimización del proceso.

(e) El desarrollo de sensores inteligentes, etc.

Esto motiva y justifica el alcance de este trabajo de investigación: el estudio del problema de estimación y control en línea en reactores de copolimerización mediante la teoria de control (geométrico) no lineal.

\subsection{Antecedentes}

Actualmente, la industria tiende hacia la adopción de tecnologias avanzadas de automatización (ya sea monitoreo y/o control), sin embargo el progreso en reactores de polimerización ha sido relativamente lento. Esto ha sido atribuido a diversos factores, tales como:

(a) La falta de instrumentos en linea robustos y confiables (Ponnuswamy et al., 1987).

(b) El insuficiente entendimiento de la dinámica del proceso.

(c) La alta sensibilidad a cambios en condiciones de operación, perturbaciones, y parámetros del proceso.

(d) El comportamiento complejo no lineal de estos reactores (posible inestabilidad o multiplicidad de estados estacionarios).

(e) El desarrollo de diseños robustos de control no lineal, que además como paso necesario requieren del diseño de estimadores de estado.

A continuación se hace una reseña de los principales estudios que se han realizado en estimación, control y selección de sensores en línea en reactores de polimerización. 


\subsubsection{Estimación de estados}

Varios investigadores han realizado revisiones sobre el estado del arte en la teoria de estimación no lineal con aplicación a procesos químicos en general (Elicabe y Meira, 1988; Bequette, 1991; Valluri y Soroush, 1996; Wang et al., 1997; Doyle, 1998, Alvarez y López, 1999). De estos trabajos se puede concluir que las técnicas de estimación principales (más empleadas) disponibles en literatura son las siguientes:

I. Observador a lazo abierto -OLA- : Este observador es por supuesto el que tiene el diseño más directo. La condición necesaria para su empleo es que, la dinámica de todos los estados (observador completo) o de algunos de ellos (observador reducido o detector) tenga un comportamiento asintóticamente estable. El principal inconveniente es que la dinámica del error de estimación no es ajustable, y por lo tanto debe aceptarse el tiempo natural de asentamiento del proceso como tiempo de convergencia. Está técnica ha sido empleada con modelos de estado de orden reducido para reactores químicos (Daoutidis y Kravaris, 1994; Soroush, 1997; Tatiraju y Soroush, 1997).

II. Filtro extendido de Kalman -FEK- (Jazwinski, 1970; Gelb, 1974): Esta técnica (estocástica) es la más empleada en ingenieria quimica, cuyo diseño es simple, y basado en la minimización de la matriz de covarianza del error de estimación. No obstante, su diseño presenta algunas desventajas: solo es aplicable a plantas completamente observables, no garantiza estabilidad, carece de criterio de sintonización (ya que el ajuste de sus parámetros se realiza a prueba y error), y el método requiere de $n(n+1) / 2$ ecuaciones diferenciales - tipo Ricatti - adicionales para una planta $n$-dimensional (éste último ya no debe considerarse como un problema, debido a que actualmente se cuenta con métodos numéricos y software suficientemente robustos para su resolución). En procesos de polimerización, el FEK ha mostrado resultados aceptables y satisfactorios tanto teórica como experimentalmente (Jo y Bankoff, 1976; Hyun et al., 1976; Schuler y Suzhen, 1985; Schuler y Papadopolou, 1986; Adebekun y Schork, 1989b; Dimitratos et al., 1991; Kozub y McGregor, 1992; Ellis et al., 1988, 1994; Mutha et al., 1997a). Sin embargo el buen desempeño del FEK está limitado. En algunos casos éste depende, en buena medida, del tipo de modelo empleado (simple o complejo); por ejemplo, Schuler y Suzhen (1985) obtuvieron ineficiencia en las predicciones (para un reactor de homopolimerización en solución) al emplear un modelo complejo junto con errores de modelado. Por otro lado, Dimitratos et al. (1991) logran desempeños aceptables para un reactor de copolimerización en emulsión, a pesar de emplear un modelo simplificado tanto para la implementación del diseño estándar del FEK como para la implementación de un diseño modificado (con matrices del error de covarianza variables en lugar de asumirlas constantes). En otra situación, Kozub y MacGregor (1992) proponen el empleo de dos FEK consecutivos para mejorar el desempeño 
lento que se obtiene cuando se emplea un solo filtro (lo cual ocurre debido a que no se tienen establecido un criterio de sintonización - velocidad de convergencia), actuando el segundo filtro como una acción integral para la compensación de errores.

III. Observador Geométrico -OG- (Bestle y Zeitz, 1983; Krener e Isidori, 1983; Krener y Respondek, 1985; Xia y Gao, 1989): Este método de estimación está basado en la noción de linealización mediante un cambio de coordenadas, adoptada de la teoria de control no lineal (Isidori, 1995). Esta técnica garantiza convergencia (más no robustez) con una dinámica del error de salida lineal, lo cual permite que los polos de su ecuación caracteristica puedan ser asignados. Sin embargo, el buen comportamiento de la dinámica asociada al error de predicción de las salidas (en términos del cambio de coordenadas) viene a ser una limitante: la clase de plantas a las que se aplica está muy restringida, y es imprescindible la resolución de $\mathrm{n}$ ecuaciones diferenciales parciales acopladas. Pocos son los sistemas en ingenieria química que admiten solución a este problema, entre ellos los trabajos de Kantor (1989) y Limqueco et al. (1991).

IV. Observador de alta ganancia -OAG- (Tsinias 1989; Gauthier et al.; 1992; Deza et al., 1992, 1993; Ciccarella et al., 1993): Este observador determinístico está derivado del observador de Luenberger (Luenberger, 1961, 1971), el cual emplea ganancias constantes. Su sintonización se realiza mediante una técnica de alta ganancia para controlar la velocidad de convergencia; sin embargo, el procedimiento es complejo. Este observador ha sido empleado en reactores biológicos y de polimerización (Van Dootingh et al., 1992; Dochain, 1992).

V. Observador de modo deslizamiento -OMD- (Slotine et al., 1987; Walcott et al., 1987): Se construye a partir de superficies deslizantes (cuya metodologia se basa en una simplificación de notación, la cual consiste en remplazar un sistema de n-ésimo orden por uno estable de primer orden), y alternando ganancias constantes. Esta técnica garantiza estabilidad robusta, pero tiene un diseño complicado. Entre los trabajos de aplicación de este observador está el estudio realizado por Wang et al. (1997), para dos reactores continuos en serie con cinética de primer orden.

Las técnicas antes mencionadas están restringidas a plantas no lineales completamente observables (Krener y Respondek, 1985), y su extensión a plantas detectables (esto es, parcialmente observables) no es simple, como muestra el trabajo de Tsinias (1990).

En resumen, en ingenieria de reactores de polimerización, el FEK es la técnica de estimación más empleada. Sin embargo, ésta y las otras cuatro técnicas descritas 
son restrictivas o presentan desventajas para su implementación. De aquí surge la necesidad de desarrollar estimadores de estados (para plantas observables o detectables), que sean: robustos, simples y sistemáticos en construcción sintonización, y que garanticen estabilidad.

\subsubsection{Control avanzado}

El problema de control en reactores de polimerización ha sido estudiado mediante el empleo de diversos tipos de control: lineal (convencional), lineal multivariable, no lineal, prealimentado, con retroalimentación de estados, o con retroalimentación de salidas. No obstante, pocos son los trabajos que se reportan con aplicación en la industria (Congalidis et al., 1989; Ellis et al., 1994; Arzamendi et al., 1991; Congalidis y Richards, 1998).

Los diseños de control lineal (Congalidis et al., 1989; Arzamendi et al., 1991), construidos mediante una linealización local del sistema no lineal, no son recomendables para sistemas fuertemente no lineales, debido a que muestran problemas de estabilidad, robustez y desempeño. Esto ha motivado al uso de la teoria del control geométrico no lineal (Wonham, 1985; Isidori, 1995; Nijmeijer y Van der Shaft, 1990), de acuerdo a la cual el problema de control debe abordarse en dos etapas (de igual importancia):

(a) una etapa de análisis, donde el énfasis está en derivar, a priori, condiciones de resolubilidad verificables (que deben ser necesarias para que exista solución al problema de control), y

(b) una etapa de diseño, la cual consiste en construir, sintonizar, e implementar el controlador de forma sistemática.

Entre las aplicaciones del control geométrico no lineal están los trabajos de: Kravarish y Soroush (1990), en el cual se construye un controlador con dinámica del error de salida de segundo orden para un reactor de copolimerización por lotes; Padilla et al. (1991), en el cual se aborda el problema del control de un reactor continuo de copolimerización empleando una extensión dinámica (que consiste en una redefinición de estados y variables de control); y Padilla et al. (1995, 1997), en las cuales se estudia el problema del control con retroalimentación de estados en reactores de copolimerización. No obstante, en estos trabajos se considera que todos los estados son conocidos, lo cual en la prâctica no sucede. Una solución a este problema consiste en acoplar el sistema de control con un estimador de estados en linea. Entre los trabajos que consideran esta estructura están: Adebekun y Schork (1989), Kozub y MacGregor (1992), Ellis et al. (1994), y Mutha et al. (1997b), en los que se considera la combinación de un controlador con un FEK; Soroush y Kravaris 
(1992, 1994), que emplean un control geométrico no lineal en combinación con un observador de orden reducido; y Alvarez (1996), que presenta un esquema de un control geométrico no lineal con un estimador de estados no lineal.

Es importante mencionar que la selección del controlador y estimador no es trivial. En sistemas lineales, el principio de separación (Wonham, 1985) garantiza la estabilidad del controlador-estimador a lazo cerrado; sin embargo, este principio no es aplicable a sistemas no lineales. A pesar de ello, existen algunos resultados que permiten garantizar la estabilidad y dar criterios de convergencia para el caso no lineal: condiciones de estabilidad asintótica local y global (Vidyasagar, 1978) mediante el empleo de la teoria de estabilidad de Lyapunov, condiciones de detectabilidad (Byrnes et al., 1991; Soroush, 1997), etc.

\subsubsection{Sensores en linea}

La selección de las mediciones no es trivial, ésta depende de la propiedad de observabilidad/detectabilidad de la planta. Esta propiedad es una relación entre las mediciones disponibles y los estados del modelo que pueden determinarse a partir de dicha relación (la definición formal se dará en el capitulo 3). Por ejemplo para el caso de reactores de homopolimerización (Ray, 1986), se conoce que a partir de mediciones de temperatura es posible inferir la conversión y/o la concentración del iniciador (ya que los cambios en temperatura se relacionan directamente con la velocidad de reacción, la cual a su vez depende de dos factores: la concentración de monómero - equivalente a la conversión- y de la concentración de iniciador). Esto mismo puede aplicarse a otras mediciones como se resume en la Tabla 1.1, lo cual esta basado en una linealización del modelo y pruebas de observabilidad lineal.

Tabla 1.1 Propiedad de observabilidad (O) o detectabilidad (D) en reactores de homopolimerización, en función de las variables medidas (Ray, 1986)

\begin{tabular}{lcccc}
\hline${ }^{*}$ Medición & pom & $I$ & $M_{n}$ & $M_{w}$ \\
\hline $\mathrm{T}$ & $\mathrm{O}$ & $\mathrm{O}$ & $\mathrm{D}$ & $\mathrm{D}$ \\
$\mathrm{T}, \eta$ & $\mathrm{O}$ & $\mathrm{O}$ & $\mathrm{D}$ & $\mathrm{D}$ \\
$\mathrm{T}, \rho$ & $\mathrm{O}$ & $\mathrm{O}$ & $\mathrm{D}$ & $\mathrm{D}$ \\
$\mathrm{T}, \eta \circ \rho, \mu$ & $\mathrm{O}$ & $\mathrm{O}$ & $\mathrm{O} / \mathrm{D}$ & $\mathrm{O}$ \\
$\mathrm{T}, \eta \circ \rho, \mathrm{GPC}$ & $\mathrm{O}$ & $\mathrm{O}$ & $\mathrm{O}$ & $\mathrm{O}$ \\
\hline
\end{tabular}

* p: conversión, m: concentración del monómero, I: concentración del iniciador, $\mathrm{M}_{\mathrm{n}}$ : peso molecular promedio-número, $\mathrm{M}_{\mathrm{w}}$ : peso molecular promedio-peso. 
De acuerdo a lo anterior, el estudio y evaluación de sensores en línea para reactores de polimerización es un tema de investigación importante. En algunos trabajos se ha hecho revisiones extensas sobre posibles instrumentos de medición (MacGregor y Tidwell, 1980; Hoogendoorn y Shaw, 1980; Ponnuswamy y Shah, 1986; Ray, 1986; Chien y Penlidis, 1990; Schork, 1991), mostrando sus características, ventajas, desventajas, e implicaciones de su empleo para esquemas de estimación y/o control. Entre los instrumentos propuestos (tanto de señales continuas como discretas) para reactores de polimerización están los siguientes: indicadores de temperatura, presión y volumen, densimetros, viscosimetros, tensiómetros, refractómetros, medidores del torque de agitación, dispersores de luz, cromatógrafos de gas y permeación gel, etc.

Otros trabajos (experimentales) proponen estrategias de inferencia (sin adoptar una técnica de estimación o control) para la conversión y composición de homo- y copolimeros, mediante el uso de mediciones secundarias y el desarrollo de expresiones que relacionan dicha medición con la conversión y/o composición. Por ejemplo, Bahr y Pinto (1991) emplean el indice de refracción como medición indirecta, Canegallo et al. (1993) emplean la densidad, Kontogeorgis y Fredeslund (1994) emplean la presión de vapor, Henderson (1987) emplea la viscosidad, y Crowley y Choi (1995) emplean la constante dieléctrica.

En la mayoria de los trabajos mencionados en las dos subsecciones anteriores (y resumidos en la Tabla 1.2), las variables medidas han sido principalmente la temperatura, la conversión mediante el indice de refracción, las concentraciones de monómeros residuales y la distribución de pesos moleculares por medio de métodos cromatográficos. Sin embargo, las mediciones de concentraciones y de pesos moleculares no son factibles en linea, debido a que son mediciones discretas con retraso (de aproximadamente $15 \mathrm{~min}$ ), que además requieren instrumentos sofisticados. Muy pocos han empleado medidas en linea convencionales o secundarias, como son temperatura, densidad, viscosidad, presión, etc. Es por esto, que uno de los objetivos de este trabajo es probar que mediante mediciones indirectas es posible reconstruir los estados del reactor, asi como las propiedades del polimero. 
Tabla 1.2 Antecedentes de mediciones empleadas en reactores de polimerización

\begin{tabular}{|c|c|c|c|c|}
\hline & Objetivo & $\begin{array}{c}{ }^{*} \text { Mediciones } \\
\text { continuas } \\
\end{array}$ & $\begin{array}{c}{ }^{*} \text { Variables } \\
\text { inferidas/controladas }\end{array}$ & $\begin{array}{c}\text { Tipo de } \\
\text { polimerización }\end{array}$ \\
\hline $\begin{array}{l}\text { Jo y Bankoff } \\
(1976)\end{array}$ & Estimación & $\eta, \tau$ & $\mathrm{P}, \mathrm{M}_{\mathrm{w}}$ & $\begin{array}{l}\text { Homopolim./ solución } \\
\text { (reactor continuo) }\end{array}$ \\
\hline $\begin{array}{l}\text { Schuler } \\
(1980)\end{array}$ & Estimación & $\begin{array}{c}\eta, T(D P M \\
\text { discreta) }\end{array}$ & DPM & $\begin{array}{c}\text { Homopol./masa } \\
\text { (reactor continuo) }\end{array}$ \\
\hline $\begin{array}{l}\text { Schuler et al. } \\
(1985,1986)\end{array}$ & Estimación & $\eta, T$ & $\mathrm{p}, \mathrm{M}_{\mathrm{n}}, \mathrm{M}_{\mathrm{w}}$ & $\begin{array}{c}\text { Homopol./solución } \\
\text { (reactor por lotes) }\end{array}$ \\
\hline $\begin{array}{l}\text { Congalidis et al. } \\
(1989)\end{array}$ & Control & $\mathrm{m}_{1}, \mathrm{~m}_{2}, \mathrm{M}_{\mathrm{w}}, \mathrm{T}$ & $P_{r}, c, M_{w}$ & $\begin{array}{l}\text { Copolim./solución } \\
\text { (reactor continuo) }\end{array}$ \\
\hline $\begin{array}{l}\text { Adebekun y } \\
\text { Schork }(1989 a, b)\end{array}$ & $\begin{array}{c}\text { Estimación } \\
\text { y control }\end{array}$ & $\mathrm{m}, \mathrm{T}$ & $\mathrm{M}_{\mathrm{n}}, \mathrm{M}_{\mathrm{w}}$ & $\begin{array}{l}\text { Homopol./solución } \\
\text { (reactor continuo) }\end{array}$ \\
\hline $\begin{array}{l}\text { Kravaris y } \\
\text { Soroush (1990) }\end{array}$ & Control & $\mathrm{m}_{1}, \mathrm{~m}_{2}, \mathrm{M}_{\mathrm{n}}, \mathrm{T}$ & $c, M_{n}$ & $\begin{array}{l}\text { Copolim./solución } \\
\text { (reactor por lotes) }\end{array}$ \\
\hline $\begin{array}{l}\text { Dimitratos et al. } \\
\text { (1991) }\end{array}$ & Estimación & $\mathrm{T}, \mathrm{m}_{1}, \mathrm{~m}_{2}$ & $c, M_{w}$ & $\begin{array}{l}\text { Copolim./emulsión } \\
\text { (reactor semicontinuo) }\end{array}$ \\
\hline $\begin{array}{l}\text { Kozub y McGregor } \\
(1992 a, 1992 b)\end{array}$ & \begin{tabular}{|c|} 
Estimación \\
y control
\end{tabular} & $\mathrm{m}_{1}, \mathrm{~m}_{2}, \mathrm{D}_{\mathrm{p}}$ & $c, M_{w}$ & $\begin{array}{c}\text { Copolim./emulsión } \\
\text { (semicontinuo) }\end{array}$ \\
\hline $\begin{array}{l}\text { Leiza et al. } \\
(1993)\end{array}$ & Control & $\mathrm{m}_{1}, \mathrm{~m}_{2}, \mathrm{D}_{\mathrm{p}}$ & $c, N_{p}$ & $\begin{array}{c}\text { Copolim./emulsión } \\
\text { (semicontinuo) }\end{array}$ \\
\hline $\begin{array}{l}\text { Ellis et al. } \\
(1988,1994)\end{array}$ & $\begin{array}{c}\text { Estimación } \\
\text { y control }\end{array}$ & $\begin{array}{c}\mathrm{T}, \rho(\mathrm{DPM} \\
\text { discreta) }\end{array}$ & $\mathrm{p}, \mathrm{I}, \mathrm{DPM}$ & $\begin{array}{l}\text { Homopol./solución } \\
\text { (reactor por lotes) }\end{array}$ \\
\hline $\begin{array}{l}\text { Soroush y } \\
\text { Kravaris (1994) }\end{array}$ & $\begin{array}{c}\text { Estimación } \\
\text { y control }\end{array}$ & $\rho, \mathrm{T}$ & $\mathrm{m}, \mathrm{I}, \mathrm{S}$ & $\begin{array}{l}\text { Homopol./solución } \\
\text { (reactor continuo) }\end{array}$ \\
\hline $\begin{array}{l}\text { Tatiraju y } \\
\text { Soroush (1997) }\end{array}$ & Estimación & $\mu, \rho, T$ & $\mathrm{I}, \mathrm{M}_{\mathrm{n}}, \mathrm{M}_{\mathrm{w}}$ & $\begin{array}{l}\text { Homopol./solución } \\
\text { (reactor continuo) }\end{array}$ \\
\hline $\begin{array}{l}\text { Mutha et al. } \\
(1997 \text { a) }\end{array}$ & Estimaciōn & $\begin{array}{c}\rho, T\left(c, D_{p}, \mu\right. \\
\text { discretas) }\end{array}$ & $\mathrm{p}, \mathrm{N}_{\mathrm{p}}, \mathrm{M}_{\mathrm{w}}$ & $\begin{array}{l}\text { Copolim./emulsión } \\
\text { (reactor por lotes) }\end{array}$ \\
\hline $\begin{array}{l}\text { Mutha et al. } \\
\text { (1997b) }\end{array}$ & $\begin{array}{c}\text { Estimación } \\
\text { y control }\end{array}$ & $H, \rho, \mu$ & $\mathrm{c}, \mathrm{M}_{\mathrm{w}}$ & $\begin{array}{c}\text { Homopol./solución } \\
\text { (reactor continuo) }\end{array}$ \\
\hline $\begin{array}{l}\text { Padilla y Alvarez } \\
(1997)\end{array}$ & Control & $\mathrm{m}_{1}, \mathrm{~m}_{2}, \mathrm{~s}, \mathrm{~T}$ & $\mathrm{P}_{\mathrm{r}}, \mathrm{c}, \mathrm{p}, \mathrm{M}_{\mathrm{w}}$ & $\begin{array}{l}\text { Copolim./ solución } \\
\text { (reactor continuo) }\end{array}$ \\
\hline $\begin{array}{l}\text { Congalidis y } \\
\text { Richards (1998) }\end{array}$ & Control & $\begin{array}{l}\mathrm{m}_{1}, \mathrm{~m}_{2}, \mathrm{~m}_{3}, \\
\mu \text { (discretas) }\end{array}$ & $c, \mu$ & $\begin{array}{l}\text { Terpolim./emulsión } \\
\text { (reactor continuo) }\end{array}$ \\
\hline
\end{tabular}

* c: composición instantánea del copolímero o terpolimero, $\mathrm{D}_{\mathrm{p}}$ : tamaño de particula, DPM: distribución de pesos moleculares, $\mathrm{H}$ : calor liberado, I: concentración del iniciador, $\mathrm{p}$ : conversión, $\mathrm{m}_{\mathrm{i}}$ : concentración del i-ésimo monómero, $\mathrm{M}_{\mathrm{w}}$ : peso molecular promedio-peso, $\mathrm{M}_{\mathrm{n}}$ : peso molecular promedio-número, $\mathrm{N}_{\mathrm{p}}$ : número de partículas, $P_{r}$ : velocidad de producción, $S$ : concentración de solvente, $T$ : temperatura, $\eta$ : indice de refracción, $\mu$ : viscosidad, $\rho$ : densidad, $\tau$ : torque del agitador. 


\subsection{Objetivo y aportacio nes}

Los problemas anteriores motivan y justifican el desarrollo de esquemas de estimación y control no lineales robustos (que garanticen estabilidad con criterios de convergencia) para reactores continuos de copolimerización en solución. Por lo cual, en esta tesis se presenta una metodologia para el diseño de estimadores y controladores (geométricos) no lineales que permitan inferir propiedades no medibles en tiempo real (como son la fracción de sólidos, la composición instantánea, la conversión, el peso molecular peso-promedio, y la velocidad de producción del copolimero), empleando un modelo dinámico del proceso en combinación con una retroalimentación de salidas medibles en linea (seleccionando mediciones continuas, instantáneas y de bajo costo, como son densidad, indice de refracción, temperatura, y volumen).

La metodologia propuesta aborda principalmente tres aspectos de estudio:

Primero, el estudio de los reactores continuos de copolimerización en solución. Donde la finalidad es describir, entender, caracterizar e interpretar el comportamiento dinámico del reactor mediante un sistema de ecuaciones diferenciales no lineales. De forma particular, como ejemplo de aplicación, se considerará el sistema de copolimerización metil metacrilato - acetato de vinilo, en acetato de etilo como solvente, y AIBN (azo-bis-isobutonitrilo) como iniciador. Este sistema de copolimerización se ha seleccionado ya que los comonómeros presentan una diferencia significativa en reactividades y en efecto gel (lo cual significa tendencia a inestabilidad, y condiciones severas de operación). Además, este sistema a lazo abierto ha sido estudiado exhaustivamente, tanto teórica como experimentalmente (lo cual permite validar el modelo del reactor desarrollado en este trabajo).

Segundo, el esquema de estimación no lineal a lazo abierto. En el cual se desarrolla un estimador geométrico no lineal, con las siguientes características: (a) construcción sistemática, (b) diseño con criterio de convergencia, (c) técnica de sintonización simple, sistemática (mediante la asignación de polos, de forma similar a las técnicas de control y filtrado lineal convencional), y con interpretación física, y (d) la estructura de estimabilidad (i.e., grado de observabilidad y partición de los estados observables - no observables) es un grado de libertad del diseño.

Tercero, el esquema de control no lineal con retroalimentación de mediciones. En el cual se propone un controlador construido a partir de la combinación del estimador de estados a lazo abierto, con el control no lineal con retroalimentación de estados; mostrando características similares al estimador (en cuanto a diseño, construcción, sintonización, y convergencia). 
Para implementación de los esquemas de estimación y de control propuestos, se han seleccionado como mediciones en linea a la densidad, el índice de refracción, la temperatura y el volumen. Con el objetivo de inferir y regular las variables de calidad (conversión, composición instantánea, y peso molecular promedio-peso del copolimero), de seguridad (fracción de sólidos y temperatura), y velocidad de producción. Con la finalidad de determinar la robustez de los esquemas propuestos, tanto el estimador como el controlador no lineal serán implementados introduciendo errores en las condiciones iniciales, cambios en las entradas exógenas, errores en los parámetros del modelo, e inyección de ruido en las mediciones.

\subsection{Organización de la tesis}

En el capitulo 2, intitulado "Reactores de copolimerización en solución”, se plantea la cinética de copolimerización y el modelo matemático para reactores continuos, semicontinuos, y por lotes. Enseguida se hace un análisis del comportamiento dinámico y en estado estacionario del reactor continuo a lazo abierto, lo cual es importante como punto de comparación para la velocidad de convergencia del estimador no lineal (puesto que este es el comportamiento de un estimador con ganancia cero, equivalente al observador a lazo abierto OLA definido en la sección 1.2.1), y para la comparación de la estabilidad del controlador no lineal (ya que se considerará la regulación de un estado estacionario inestable).

En el capítulo 3, intitulado "Estimación de estados no lineal", se presenta el estimador no lineal: (a) las condiciones de estimabilidad, que garantizan a priori (esto es, antes de simulaciones numéricas) la solución al problema de estimación, (b) la construcción, que como se ha mencionado, es simple y sistemática, (c) los criterios de convergencia, que garantizan la estabilidad del estimador (mostrando la prueba técnica de convergencia en el Apéndice F), (d) las medidas de estimabilidad, las cuales son medidas cuantitativas del desempeño-robustez del estimador, (e) la técnica de sintonización, que consiste de la asignación de polos de forma similar a sintonización de filtros lineales de una entrada y controladores lineales de una entrada - una salida, y tiene una interpretación física (definiendo tiempos de asentamiento y el factor de amortiguamiento), (f) los grados de libertad del diseño del estimador, los cuales son principalmente la estructura de estimabilidad y los parámetros de sintonización; y finalmente (g) una comparación cualitativa con el FEK, en cuanto a ventajas y desventajas de este diseño y el propuesto. 
En el capítulo 4, intitulado "Estimación de estados en reactores de copolimerización", se plantea una estrategia para la selección de la estructura de estimabilidad. Ya que de forma estricta, existen 116 posibles estructuras para el reactor de copolimerización, y con algunas consideraciones pueden reducirse hasta 22 estructuras. Despuês, en la etapa de análisis, se plantean las condiciones de resolubilidad, y las medidas de estimabilidad para las estructuras definidas. Finalmente, en la etapa de diseño, se construyen, sintonizan, e implementan cuatro estimadores (con diferentes grados de estimabilidad) para compararlos entre si, y corroborar las conjeturas realizadas en la etapa de análisis.

En el capitulo 5, intitulado "Control no lineal con retroalimentación de mediciones", primero se plantea el problema de estimación de estados, el cual debe considerarse como el 'control exacto'. Enseguida se propone un esquema de controladorestimador: su construcción, criterio de convergencia, y sintonización. Finalmente, se implementa en el reactor de copolimerizaciōn, considerando la mejor estructura de estimabilidad obtenida en el capitulo 4; que en forma similar al problema de estimación, se divide en dos etapas: anâlisis y diseño.

Por último en el Capitulo 6, intitulado "Conclusiones", se discute y resume la contribución de este trabajo de investigación, se dan algunas recomendaciones y trabajo futuro de investigación. 
INTRODUCCIÓN 


\title{
Capítulo 2
}

\section{REACTORES DE COPOLIMERIZACIÓN EN}

\author{
SOLUCIÓN
}

En este capitulo se presenta el modelo matemático para la copolimerización en solución via radicales libres (para reactores continuos, semicontinuos, o por lotes). La finalidad es describir, entender, caracterizar e interpretar el comportamiento dinámico del reactor mediante un sistema de ecuaciones diferenciales no lineales. Para ello, primero se analiza el comportamiento alrededor de estados estacionarios y luego de trayectorias. Este análisis es importante como punto de comparación para la velocidad de convergencia del estimador no lineal, del desempeño y estabilidad del controlador no lineal que se construirán en los siguientes capitulos. 


\subsection{Modelo matemático del reactor}

El objetivo de este capitulo es establecer el modelo del reactor de copolimerización en solución, vía radicales libres. El modelo de este sistema es fundamental para: (a) estudiar el comportamiento del reactor a lazo abierto, (b) diseñar la operación nominal, la cual representa una condición severa para el problema de estimación y control, (c) implementar estrategias de estimación y control geométrico no lineal, y (d) obtener el comportamiento limite alcanzable para un estimador y controlador retroalimentado. El presente modelo se obtiene a partir de principios básicos de cinética química, transferencia de calor, reologia, fisicoquímica e ingeniería de reactores de polimerización.

En la primera sección se describe el reactor y las expresiones matemáticas del modelo correspondiente. En la segunda sección se diseña la operación nominal, y se realiza un análisis del comportamiento $\mathrm{y}$ estabilidad del reactor en estado estacionario y transitorio.

\subsubsection{Balances de materia y energia.}

El desarrollo de modelos y el comportamiento dinámico de reactores de copolimerización en solución ha sido motivo de varias investigaciones (Ray, 1971, 1972; Hamer et al., 1981; Schmidt et al., 1984; Teymour y Ray, 1989, 1992; Pinto y Ray, 1995, 1996), en las que incluso se han realizado verificaciones experimentales. Un modelo puede ser sencillo o complejo dependiendo del centro de interés. Para ingenieria de reactores y control de procesos, éste debe tomar en cuenta: la cinética quimica, las propiedades de mezclado, los cambios en las propiedades físicas, y las variaciones en temperatura. Los procesos de copolimerización en solución vía radicales libres se caracterizan por tener incrementos de temperatura por la alta generación de calor, incrementos en la viscosidad que genera dificultades difusionales, y un efecto gel o "Tromsdorff" que vuelve incontrolable la reacción, aumentado la temperatura, produciendo una conversión rápida y en ocasiones taponamiento del equipo.

El modelo que aquí se emplea corresponde al desarrollado y presentado en López (1995) y Padilla (1997), el cual considera:

(i) Un mecanismo por radicales libres tomando en cuenta las etapas de iniciación, propagación y terminación (Tabla 2.1). Los efectos de transferencia de cadena a monómeros o solvente se han despreciado, por simplicidad del modelo y puesto 
que el control de la distribución de pesos moleculares no es un objetivo en este trabajo.

(ii) El efecto gel (Sharma y Soane, 1988; Hamer et al. 1981), el cual causa la disminución de las velocidades de terminación causadas por un decremento en la movilidad o difusión de las cadenas poliméricas. Este fenómeno modifica las propiedades finales del polimero: los pesos moleculares son más altos y su distribución se vuelve más ancha.

(iii) La variación de la densidad de la mezcla polimérica con respecto a la fracción de sólidos, debido a que los polímeros son invariablemente más densos que sus monómeros provocando una contracción en el volumen de reacción. Para ello se introducen un conjunto de parámetros $\left\{\varepsilon_{1}, \varepsilon_{2}, \phi_{1}, \phi_{2}, \phi_{3}\right\}$ :

$$
\begin{array}{ll}
\varepsilon_{1}=1-\rho_{1}^{m} / \rho_{1}^{P} & \\
\varepsilon_{2}=1-\rho_{2}^{m} / \rho_{2}^{p} & \\
\phi_{1}=\left(1-\varepsilon_{1}^{m}\right)\left(1-\varepsilon_{1} m_{1 e}\right) /\left(1-\varepsilon_{1}\right)+\varepsilon_{1}^{m} m_{1 e}+\varepsilon_{1}^{p} p_{1 e}, & \varepsilon_{i}^{m}=1-\rho_{i}^{m} / \rho \\
\phi_{2}=\left(1-\varepsilon_{2}^{m}\right)\left(1-\varepsilon_{2} m_{2 e}\right) /\left(1-\varepsilon_{2}\right)+\varepsilon_{2}^{m} m_{2 e}+\varepsilon_{2}^{p} p_{2 e}, & \varepsilon_{i}^{p}=1-\rho_{i}^{p} / \rho \\
\phi_{3}=1+\varepsilon^{s}\left(s_{e}-1\right), & \varepsilon^{s}=1-\rho^{s} / \rho
\end{array}
$$

donde $\varepsilon_{1}$ y $\varepsilon_{2}$ son las contracciones de volumen de los monómero puros 1 y 2 , respectivamente, referidos a la densidad del polimero correspondiente; $\phi_{1}, \phi_{2}$, y $\phi_{3}$ corresponden a las contracciones de volumen del monómero 1 , monómero $2, \mathrm{y}$ solvente en la alimentación, respectivamente, referidos a la densidad de la mezcla polimérica (ya que las materias primas pueden contener impurezas o parte del monómero prepolimerizados). En caso de que las materias primas sean puras (esto es, $m_{1 \mathrm{e}}=m_{2 \mathrm{e}}=s_{1 \mathrm{e}}=1$ y $p_{1 \mathrm{e}}=p_{2 \mathrm{e}}=0$ ), entonces $\left\{\varepsilon_{1}, \varepsilon_{2}, \phi_{1}, \phi_{2}, \phi_{3}\right\}=\left\{\varepsilon_{1}, \varepsilon_{2}\right.$, $1,1,1\}$. En el caso de densidad constante (generalmente a bajas conversiones $o$ altas concentraciones de solvente), se tiene que no hay corrección por contracción de volumen y entonces $\left\{\varepsilon_{1}, \varepsilon_{2}, \phi_{1}, \phi_{2}, \phi_{3}\right\}=\{0,0,1,1,1\}$; reduciéndose el modelo al presentado por Hamer et al. (1981).

(iv) El efecto de la variación de la viscosidad (Alvarez et al., 1994) sobre el coeficiente de transferencia de calor (Henderson, 1987), ya que un aumento en la viscosidad conduce a problemas en la velocidad de agitación, así como en una reducción en la transferencia de calor y aumento en la temperatura (con posibilidad de disparos térmicos e inestabilidad del proceso). 
Tabla 2.1 Mecanismo cinético de la copolimerización

\begin{tabular}{|c|c|c|}
\hline Iniciación & Propagación & Terminación \\
\hline$\left\{\begin{array}{l}I \stackrel{k_{d}}{\longrightarrow} 2 R \\
R+M_{1} \stackrel{k_{i 1}}{\longrightarrow} P_{1,0} \\
R+M_{2} \stackrel{k_{i 2}}{\longrightarrow} Q_{0,1}\end{array}\right.$ & $\left\{\begin{array}{l}P_{n, m}+M_{1} \stackrel{k_{p 11}}{\longrightarrow} P_{n+1, m} \\
P_{n, m}+M_{2} \stackrel{k_{p 12}}{\longrightarrow} Q_{n, m+1} \\
Q_{n, m}+M_{1} \stackrel{k_{p 21}}{\longrightarrow} P_{n+1, m} \\
Q_{n, m}+M_{2} \stackrel{k_{p 22}}{\longrightarrow} Q_{n, m+1}\end{array}\right.$ & $\left\{\begin{array}{l}P_{n, m}+P_{r, q} \stackrel{k_{i 11}}{\longrightarrow} D_{n+r, m+q} \\
P_{n, m}+Q_{r, q} \stackrel{k_{t 12}}{\longrightarrow} D_{n+r, m+q} \\
Q_{n, m}+Q_{r, q} \stackrel{k_{t 22}}{\longrightarrow} D_{n+r, m+q}\end{array}\right.$ \\
\hline
\end{tabular}

$I, R, M_{1}, M_{2}, P_{n, m}, Q_{n, m}, D_{n, m}$ representan a el iniciador, los radicales primarios, el monómero 1, el monómero 2, las cadenas poliméricas activas con $n$ unidades de $M_{1}$ y $m$ unidades de $M_{2}$ terminando con un radical activo $M_{1}$, las cadenas poliméricas activas con $n$ unidades de $M_{1}$ y $m$ unidades de $M_{2}$ terminando con un radical activo $M_{2}$, y las cadena poliméricas muertas con $n$ unidades de $M_{1}$ y $m$ unidades de $M_{2}$, respectivamente. $k_{\mathrm{d}}, k_{\mathrm{ij}}, k_{\mathrm{pj}}, k_{\mathrm{tij}}$ denotan las constantes de velocidad de disociación del iniciador, de formación de radicales primarios, de propagación y de terminación, respectivamente.

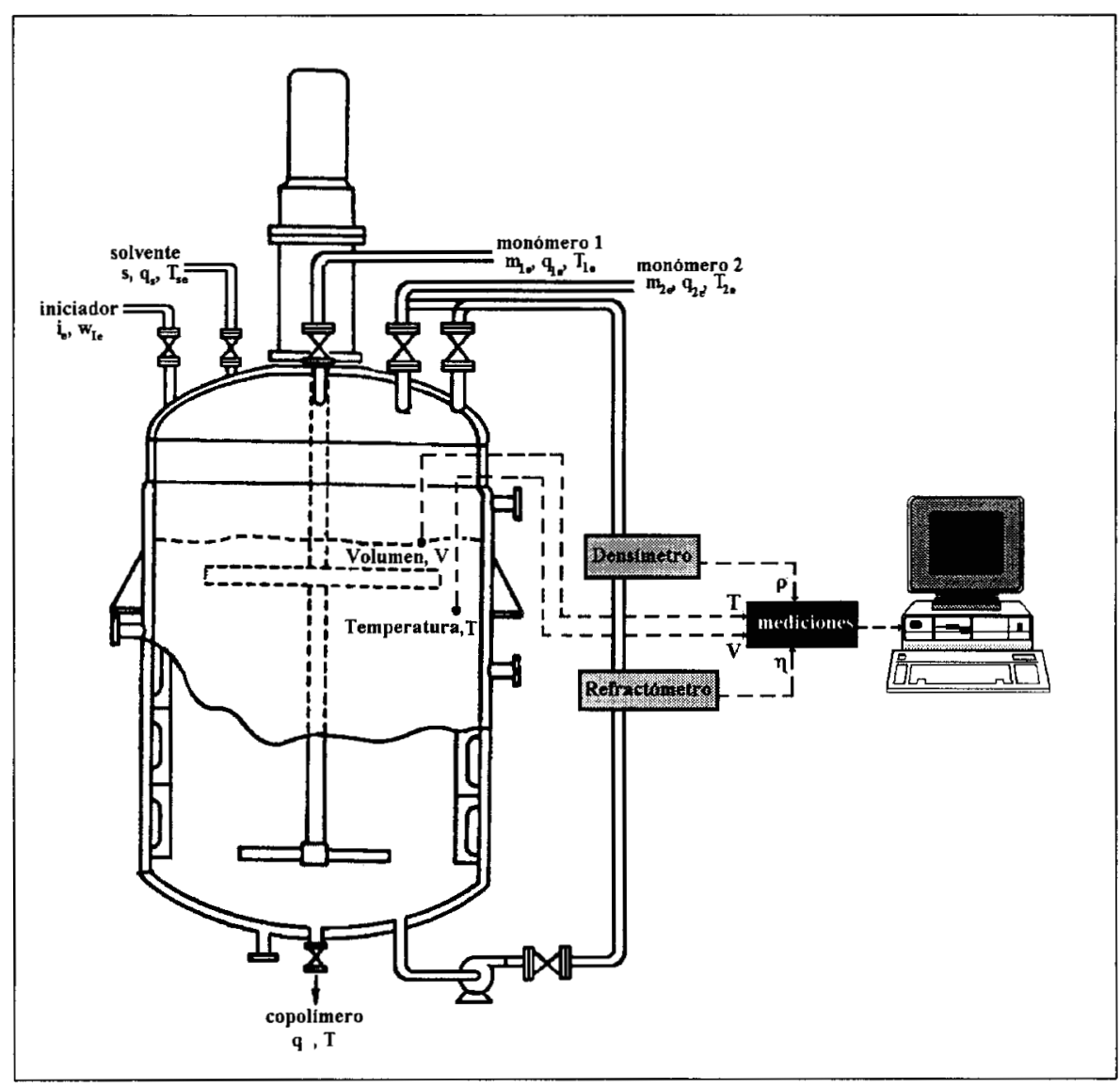

Figura 2.1 El reactor de copolimerización en solución 
El modelo que a continuación se presenta es válido para un reactor lote, semilote, o continuo tanque agitado (ver Figura 2.1) en estado transitorio, donde el calor generado por reacción es eliminado mediante una camisa de enfriamiento. Para el caso de reactores continuos en estado estacionario, el término de acumulación $(\dot{x})$ es igual a cero, generando un sistema de ecuaciones algebraicas. Para un reactor semicontinuo, los términos de salida deben eliminarse, y para un reactor por lotes, ambos términos de entrada y salida deben eliminarse. El modelo dinámico del reactor está descrito por el siguiente sistema de (nueve) ecuaciones no lineales [el detalle sobre el desarrollo del modelo puede verse en López (1995), y las funcionalidades se encuentran resumidas en el Apéndice A]:

$$
\begin{aligned}
& \dot{m}_{1}=-r_{1}\left(m_{1}, m_{2}, p_{1}, p_{2}, i, T\right)+\left(q_{1} m_{1 e}-q_{e} m_{1}\right) / V:=f_{1} \\
& \dot{m}_{2}=-r_{2}\left(m_{1}, m_{2}, p_{1}, p_{2}, i, T\right)+\left(q_{2} m_{2 e}-q_{e} m_{2}\right) / V:=f_{2} \\
& \dot{p}_{1}=\left[r_{1}\left(m_{1}, m_{2}, p_{1}, p_{2}, i, T\right)\right]\left(1-\varepsilon_{1}\right)+\left(q_{1} p_{1 e}-q_{e} p_{1}\right) / V:=f_{3} \\
& \dot{p_{2}}=\left[r_{2}\left(m_{1}, m_{2}, p_{1}, p_{2}, i, T\right)\right]\left(1-\varepsilon_{2}\right)+\left(q_{2} p_{2 e}-q_{e} p_{2}\right) / V:=f_{4} \\
& \dot{i}=-r_{I}(i, T)+\left(w_{l e} / I^{o}-q_{e} i\right) / V:=f_{5} \\
& \dot{T}=r_{T}\left(m_{1}, m_{2}, p_{1}, p_{2}, i, T\right)-\gamma(T-T c)+q_{h e}-q_{h}:=f_{6} \\
& \dot{V}=q_{e}-q:=f_{7} \\
& \dot{\mu_{0}}=r_{\mu_{0}}\left(m_{1}, m_{2}, p_{1}, p_{2}, i, T, \mu_{0}\right)+\left[\left(q_{1}+q_{2}\right) \mu_{0 e}-q_{e} \mu_{0}\right] / V:=f_{8} \\
& \dot{\mu_{2}}=r_{\mu_{2}}\left(m_{1}, m_{2}, p_{1}, p_{2}, i, T, \mu_{2}\right)+\left[\left(q_{1}+q_{2}\right) \mu_{2 e}-q_{e} \mu_{2}\right] / V:=f_{9}
\end{aligned}
$$

donde el flujo $q_{e}$

$$
q_{e}=q_{1} \phi_{1}+q_{2} \phi_{2}+q_{s} \phi_{3}-\left(r_{1} \varepsilon_{1}+r_{2} \varepsilon_{2}\right) V
$$

corresponde al flujo de alimentación corregido por la variación de la densidad, y como se mencionó anteriormente si la densidad es constante entonces $\left\{\varepsilon_{1}, \varepsilon_{2}, \phi_{1}, \phi_{1}\right.$, $\left.\phi_{3}\right\}=\{1,1,1,0,0\}$ y $q_{e}=q_{1}+q_{2}+q_{s}$ (con lo cual el modelo se reduce al presentado por Hamer et al., 1981).

Los estados $(x)$ del reactor son: las concentraciones adimensionales referidas a los componentes puros (ver definición en Apéndice A) de monómero $1\left(m_{1}\right)$, monómero 2 $\left(m_{2}\right)$, monómero convertido $1\left(p_{1}\right)$, monómero convertido $2\left(p_{2}\right)$, e iniciador (i); la temperatura $(T)$, el volumen $(\eta)$, el momento cero $\left(\mu_{0}\right)$ y el segundo momento $\left(\mu_{2}\right)$ de la distribución de longitud de cadena (DLC). Cabe señalar que la concentración de 
solvente ( $s$ ) no se ha incluido como un estado dinámico ya que la suma de las concentraciones adimensionales de los monómeros remanentes, de los monómeros convertidos, y del solvente es igual a uno; y por tanto

$$
s=1-\left(m_{1}+m_{2}+p_{1}+p_{2}\right)
$$

Las entradas de control (u) son: los flujos volumétricos de alimentación de los monómeros $\left(q_{1}, q_{2}\right)$, el flujo volumétrico de descarga $(q)$, y la temperatura de la camisa $\left(T_{\mathrm{c}}\right)$.

Las entradas exógenas (d) son: las concentraciones de alimentación de los monómeros puros y convertidos $\left(m_{1 \mathrm{e}}, m_{2 \mathrm{e}}, p_{1 \mathrm{e}}, p_{2 \mathrm{e}}\right)$, las temperaturas de entrada de los monómeros y solvente $\left(T_{1 \mathrm{e}}, T_{2 \mathrm{e}}, T_{\mathrm{se}}\right)$, el flujo volumétrico de alimentación del solvente $\left(q_{\mathrm{s}}\right)$, y el flujo másico de alimentación del iniciador $\left(w_{\mathrm{ie}}\right)$.

Las funciones escalares $r_{\mathrm{l}}, r_{\mathrm{i}}, \quad r_{\mu \mathrm{i}}, \mathrm{y} \quad r_{\mathrm{T}}$ corresponden a las velocidades de descomposición del iniciador, de conversión del $i$-ésimo monómero, de generación del $i$-ésimo momento de la DLC, y de intercambio de calor, respectivamente.

\subsubsection{Mediciones en linea}

Para monitorear y controlar el reactor se dispone de medidas (en linea) de la temperatura $\left(y_{\mathrm{T}}\right)$ mediante un termopar o RTD, el volumen $\left(y_{\mathrm{v}}\right)$ mediante un sensor de nivel, la densidad $\left(y_{p}\right)$ mediante un densimetro, y el indice de refracción $\left(y_{\eta}\right)$ mediante un refractómetro:

$$
y=\left(y_{p}, y_{\eta}, y_{T}, y_{V}\right)^{\prime}
$$

Estas mediciones han sido utilizadas exitosamente en varios estudios de estimación en linea para reactores de polimerización (Jo y Bankoff, 1976; Ray, 1986; Ponnuswamy y Shah, 1986; Schuler y Suzhen, 1985; Canegallo et al., 1993; Ellis et al., 1994).

El cálculo de la densidad $(\rho)$ es obtenido a partir de la aditividad de volúmenes:

$$
v=w_{1}^{m} v_{1}^{m}+w_{2}^{m} v_{2}^{m}+w_{1}^{p} v_{1}^{p}+w_{2}^{p} v_{2}^{p}+w_{s} v_{s}, \quad \text { o bien } \quad \frac{1}{\rho}=\frac{w_{1}^{m}}{\rho_{1}^{m}}+\frac{w_{2}^{m}}{\rho_{2}^{m}}+\frac{w_{1}^{p}}{\rho_{1}^{p}}+\frac{w_{2}^{p}}{\rho_{2}^{p}}+\frac{w^{s}}{\rho^{s}}
$$

Donde $v_{\mathrm{j}}^{\mathrm{i}}, w_{\mathrm{j}}^{\mathrm{i}}$, y $\rho_{\mathrm{j}}^{\mathrm{i}}$ son los volúmenes especificos, las fracciones masa, y las densidades de los componentes puros en la mezcla polimérica. Para propósitos prácticos se considera que la mezcla polimérica es ideal e independiente de la temperatura (ya que la variación de la densidad con la temperatura es pequeña, 
comparada con la variación con respecto a la concentración. La expresión para la densidad usada (López, 1995) es la siguiente:

$$
\rho\left(m_{1}, m_{2}, p_{1}, p_{2}\right)=m_{1}\left(\rho_{1}^{m}-\rho^{s}\right)+m_{2}\left(\rho_{2}^{m}-\rho^{s}\right)+p_{1}\left(\rho_{1}^{p}-\rho^{s}\right)+p_{2}\left(\rho_{2}^{p}-\rho^{s}\right)
$$

donde $\rho_{1}^{\mathrm{m}}, \rho_{2}^{\mathrm{m}}, \rho_{1}^{\mathrm{p}}, \rho_{2}^{\mathrm{p}}, \rho^{\mathrm{s}}$ son las densidades de los monómeros puros, solvente, y polímeros, respectivamente.

La dependencia del indice de refracción $(\eta)$ en los estados del reactor se establece con la teoria de Lorimer (Lorimer, 1972a, 1972b; Lorimer y Jones, 1972) para polimeros en solución. En ésta se toma en cuenta los cambios de volumen, los efectos de polidispersidad, las concentraciones y refracciones especificas parciales de cada uno de los polimeros en solución, de acuerdo a la expresión (Lorimer, 1972b):

$$
\frac{\Delta \eta}{c_{s}} \equiv \frac{\eta-\eta_{s}}{c_{s}}=v+a_{2} c_{s}
$$

Donde $\eta$ es el indice de refracción de una solución conteniendo un polímero de concentración $c_{\mathrm{s}}, \eta_{\mathrm{s}}$ es el indice de refracción del solvente, $\mathrm{v}$ es el incremento del indice de refracción específico a dilución infinita, y $a_{2}$ depende de la longitud de onda y de la temperatura. Para un copolimero en solución, el índice de refracción está dado por:

$$
\begin{gathered}
\eta\left(\mathrm{m}_{1}, \mathrm{~m}_{2}, \mathrm{p}_{1}, \mathrm{p}_{2}\right)=\eta_{o}+p_{1} v_{1} \rho_{1}^{p}+p_{2} v_{2} \rho_{2}^{p}+a_{2}\left(p_{1} \rho_{1}^{p}+p_{2} \rho_{2}^{p}\right)^{2} \\
\eta_{\mathrm{o}}=\frac{m_{1}\left(\eta_{1}-\eta_{\mathrm{s}}\right)+m_{2}\left(\eta_{2}-\eta_{\mathrm{s}}\right)+\left(1-p_{1}-p_{2}\right) \eta_{\mathrm{s}}}{1-p_{1}-p_{2}}, \quad a_{2}=\left(\frac{3 \eta_{o}^{2}-2}{2 \eta_{o}^{3}+4 \eta_{o}}\right)\left(\frac{p_{1} v_{1} \rho_{1}^{p}+p_{2} v_{2} \rho_{2}^{p}}{p_{1} \rho_{1}^{p}+p_{2} \rho_{2}^{p}}\right)^{2}
\end{gathered}
$$

Las dependencias del par $(\rho, \eta)$ con respecto a la temperatura no se tomaron en cuenta, ni las propiedades en exceso. No obstante, en caso necesario (para estudios más rigurosos y con otros fines de entendimiento) se pueden incluir, sin que esto modifique las estrategias de estimación y control que se presentan en el presente trabajo de investigación.

\subsubsection{Variables de calidad}

Las salidas de interés $(z)$ o variables de calidad son: la fracción de sólidos $\left(z_{\mathrm{s}}\right)$, la composición instantánea del monómero $1\left(z_{\mathrm{c}}\right)$, la conversión del copolímero $\left(z_{\mathrm{p}}\right)$, el peso molecular promedio-peso $\left(z_{\mathrm{M}}\right)$ de la DLC, y la velocidad de producción de copolimero $\left(z_{R}\right)$ :

$$
z=\left(z_{s}, z_{c}, z_{p}, z_{M}, z_{R}\right)^{\prime}
$$


Estas variables son clave en el monitoreo del nivel de seguridad, operabilidad, producción, y calidad de un reactor industrial; y están definidas como:

$$
\begin{aligned}
& m_{s}=\frac{\text { masa de polímero formado }}{\text { masa de monómero remante }+ \text { masa de polímero formado }+ \text { masa de solvente }} \\
& c=\frac{\text { velocidad de crecimiento del polímero } 1}{\text { velocidad de crecimiento del polímero } 1+\text { velocidad de crecimiento del polímero } 2} \\
& p=\frac{\text { moles de polimero formado }}{\text { moles de monómero remante }+ \text { moles de polímero formado }} \\
& R=\text { velocidad de formación de polímero, en } \mathrm{Kg} / \mathrm{h} \\
& M=\frac{\text { segundo momento de la } \mathrm{DLC}}{\text { primer momento de la } \mathrm{DLC}}
\end{aligned}
$$

Correspondiendo a las siguientes expresiones:

$$
\begin{aligned}
& m_{s}\left(m_{1}, m_{2}, p_{1}, p_{2}\right)=\frac{p_{1} \rho_{1}^{o}+p_{2} \rho_{2}^{o}}{m_{1}\left(\rho_{1}^{o}-\rho_{s}^{o}\right)+m_{2}\left(\rho_{2}^{o}-\rho_{s}^{o}\right)+p_{1}\left(\rho_{1}^{p}-\rho_{s}^{o}\right)+p_{2}\left(\rho_{2}^{p}-\rho_{s}^{o}\right)+\rho_{s}^{o}} \\
& c\left(m_{1}, m_{2}, p_{1}, p_{2}, i, T\right)=\frac{r_{1}\left(m_{1}, m_{2}, p_{1}, p_{2}, i, T\right) M_{1}^{o}}{r_{1}\left(m_{1}, m_{2}, p_{1}, p_{2}, i, T\right) M_{1}^{o}+r_{2}\left(m_{1}, m_{2}, p_{1}, p_{2}, i, T\right) M_{2}^{o}} \\
& p\left(m_{1}, m_{2}, p_{1}, p_{2}\right)=\frac{p_{1} P_{1}^{o}+p_{2} P_{2}^{o}}{m_{1} M_{1}^{o}+m_{2} M_{2}^{o}+p_{1} P_{1}^{o}+p_{2} P_{2}^{o}} \\
& R\left(m_{1}, m_{2}, p_{1}, p_{2}, i, T\right)=\left[r_{1}\left(m_{1}, m_{2}, p_{1}, p_{2}, i, T\right) \rho_{1}^{o}+r_{2}\left(m_{1}, m_{2}, p_{1}, p_{2}, i, T\right) \rho_{2}^{o}\right] V \\
& M\left(m_{1}, m_{2}, p_{1}, p_{2}, \mu_{2}\right)=\frac{\mu_{2}}{p_{1} P_{1}^{o}+p_{2} P_{2}^{o}}
\end{aligned}
$$

En caso de requerirse el peso molecular promedio-número $(N=$ primer momento de la $D L C /$ momento cero de la $D L C)$ o la polidispersidad $(Q=M / N)$, éstos se calculan mediante las siguientes expresiones:

$$
\begin{aligned}
& N\left(m_{1}, m_{2}, p_{1}, p_{2}, \mu_{2}\right)=\frac{p_{1} P_{1}^{o}+p_{2} P_{2}^{o}}{\mu_{0}} \\
& Q\left(m_{1}, m_{2}, p_{1}, p_{2}, \mu_{2}\right)=\frac{\mu_{0} \mu_{2}}{\left(p_{1} P_{1}^{o}+p_{2} P_{2}^{o}\right)^{2}}
\end{aligned}
$$




\subsubsection{El modelo dinámico}

El sistema de ecuaciones (2.2) que describen el reactor de copolimerización puede ser reescrito en la siguiente forma compacta:

$$
\begin{array}{rlll}
\dot{x}=f[x, u(t), d(t), b], & y=h(x, b), & z=h_{z}(x, b) \\
x \in X, \quad y \in Y, \quad z \in Z, & u \in U, \quad d \in D, & b \in B
\end{array}
$$

donde

$$
\begin{array}{lc}
x=\left(m_{1}, m_{2}, p_{1}, p_{2}, i, T, V, \mu_{0}, \mu_{2}\right)^{\prime}, & \operatorname{dim}(x)=9 \\
u=\left(q_{1}, q_{2}, \mathrm{~T}_{c}, \mathrm{q}\right)^{\prime}, & \operatorname{dim}(u)=4 \\
d=\left(m_{1 e}, m_{2 e}, p_{1 e}, p_{2 e}, \mathrm{~T}_{1 e}, \mathrm{~T}_{2 e}, T_{s e}, q_{s}, w_{I e}\right)^{\prime}, & \operatorname{dim}(d)=9 \\
y=\left(y_{\rho}, y_{\eta}, y_{T}, y_{V}\right)^{\prime}, & \operatorname{dim}(y)=4 \\
z=\left(z_{s}, z_{c}, z_{p}, z_{M}, z_{R}\right)^{\prime}, & \operatorname{dim}(z)=5 \\
h(x, b)=[\rho(x), \eta(x), T, V]^{\prime}, & \operatorname{dim}(h)=4 \\
h_{z}(x, d, b)=\left[m_{s}(x), c(x), p(x), M(x), R(x)\right]^{\prime}, & \operatorname{dim}\left(h_{z}\right)=5
\end{array}
$$

El vector $b$ corresponde a los parámetros del modelo (constantes cinéticas, calores de reacción, factores de contracción de volumen, etc.). Los conjuntos $X, Y, Z, U, \mathrm{y} B$ son conjuntos acotados y delimitados por consideraciones fisicas. Las entradas de control $u_{1}(t), \ldots, u_{\mathrm{m}}(t)$ y las entradas exógenas $d_{1}(t), \ldots, d_{\mathrm{p}}(t)$ son funciones del tiempo continuas por tramos, y los mapas $f, g$ y $h$ son suficientemente suaves (diferenciables) alrededor de la evolución $\mathrm{E}(t)$

$$
\begin{aligned}
& \mathrm{E}(t)=\{x(t), u(t), d(t), y(t)\} \\
& x(t)=\theta_{x}\left[t, t_{o}, x_{o}, u(t), d(t), b\right]
\end{aligned}
$$

Donde la trayectoria $x(t)$ (posiblemente inestable) está determinada únicamente por el conjunto de datos $\left\{x_{o}, u(t), d(t), \mathrm{b}\right\}$ (estados - entradas de control - entradas exógenas - parámetros iniciales).

\subsection{Análisis del compor tamiento y estabilidad del reactor}

En esta sección se considera el caso particular de la copolimerización en solución de metil metacrilato (MMA) - acetato de vinilo (AV) en acetato de etilo (AE) como solvente, empleando Azo-bis-isobutironitrilo (AIBN) como iniciador, en un reactor continuo (los valores de los parámetros del modelo, asi como la geometria del reactor se encuentran en el Apéndice B). Se escogió este sistema en particular, debido a que: 
- Su comportamiento a lazo abierto ha sido estudiado ampliamente tanto teórica como experimentalmente (Hamer et al., 1981; Schmidt et al., 1984; Teymour y Ray, 1989, 1992; Pinto y Ray, 1995a, 1995b).

- En copolimerización el MMA es mucho más reactivo que el AV, ya que la razón entre sus reactividades es $r_{\mathrm{MMA}} / r_{\mathrm{AV}} \approx 860$ (Brandrup e Immegut, 1989); lo cual significa una mayor tendencia a inestabilidad, y condiciones severas de operación.

- El reactor continuo puede presentar multiplicidad de estados estacionarios, estables o inestables (lo cual nos permite someter los esquemas de estimación y control a una prueba drástica de operación).

- Este sistema ha sido motivo de algunos estudios de control (Congalidis et al., 1989; Kravaris y Soroush, 1990; Padilla, 1995), los cuales se puede tener como referencias para el desempeño del controlador que en este trabajo de investigación se propone.

Las condiciones de operación del reactor continuo en estado estacionario se diseñaron tomado como referencia el reactor estudiado por Congalidis et al. (1989), y modificándolo para obtener condiciones más severas. El estado estacionario empleado por Congalidis et al. (1989), como punto de referencia (setpoint) para control, corresponde a:

(1) Concentraciones adimensionales $\left(m_{1}, m_{2}, p_{1}, p_{2}, s\right)=(0.0647,0.5535,0.0791$, $0.0526,0.02501)$

(2) conversión del $20 \%$ (con lo cual asumen que la viscosidad es constante y moderada, y por lo tanto no consideran el efecto gel ni variaciones en el coeficiente de transporte de calor),

(3) fracción sólidos de $16.2 \%$ (con lo cual consideran densidad constante),

(4) composición de MMA en el copolímero de $56 \%$ molar,

(5) tiempo de residencia de $6 \mathrm{~h}, \mathrm{y}$

(6) un estado estacionario único y estable.

Las modificaciones a lo anterior se realizaron de tal forma que el estado estacionario alcance aproximadamente: una conversión del $45 \%$, una fracción de sólidos entre $30-40 \%$ (el máximo admisible en la práctica está entre 40 - 45\%), una composición de MMA de aproximadamente $30 \%$ (para que el efecto gel y la diferencia entre reactividades sea más evidente, además que el MMA tiene un costo mayor que el $\mathrm{AV}$ ), y un tiempo de residencia menor (aproximadamente $4 \mathrm{~h}$ ). 
Para tal operación, en continuo, la variable nominal de interés $(\bar{z})$ y el conjunto de entradas nominales $\left(\bar{u}, \bar{d}\right.$ ) diseñadas (para un volumen nominal de $2 \mathrm{~m}^{3}$ ) son las siguientes:

$$
\begin{aligned}
\bar{z} & =\left(\bar{z}_{s}, \bar{z}_{c}, \bar{z}_{p}, \bar{z}_{M}, \bar{z}_{R}\right)^{\prime} \\
& =(0.3606,0.2888,0.4439,273893 \mathrm{Kg} / \mathrm{Kmol}, 187.3 \mathrm{Kg} / \mathrm{h})^{\prime} \\
\bar{u} & =\left(\bar{q}_{1}, \bar{q}_{2}, \bar{T}_{c}, \bar{q}\right)^{\prime} \\
& =\left(0.00111 \mathrm{~m}^{3} / \mathrm{min}, 0.00623 \mathrm{~m}^{3} / \mathrm{min}, 328^{\circ} \mathrm{K}, 0.00864 \mathrm{~m}^{3} / \mathrm{min}\right)^{\prime} \\
\bar{d} & =\left(\bar{m}_{1 e}, \bar{m}_{2 e}, \bar{p}_{1 e}, \bar{p}_{2 e}, \bar{T}_{1 e}, \bar{T}_{2 e}, \bar{T}_{s e}, \bar{q}_{s}, \bar{w}_{l e}\right)^{\prime} \\
& =\left(1,1,0,0,315^{\circ} \mathrm{K}, 315^{\circ} \mathrm{K}, 315^{\circ} \mathrm{K}, 0.00199 \mathrm{~m}^{3} / \mathrm{min}, 6.66 \times 10^{-5} \mathrm{Kmol} / \mathrm{min}\right)^{\prime}
\end{aligned}
$$

el cual corresponde a un tiempo de residencia de $231.5 \mathrm{~min}(3.85 \mathrm{~h})$.

La determinación de la estabilidad de este punto critico, así como el estudio del comportamiento dinámico del reactor se realiza en las siguientes secciones.

\subsubsection{El reactor autónomo}

El estado estacionario nominal $\bar{x}$ del reactor autónomo (asociado a entradas nominales $\bar{u}$ y $\bar{d}$, y salidas nominales $\bar{y}$ y $\bar{z}$ ) satisface la versión estática de la ecuación (2.7)

$$
0=f[\bar{x}, \bar{u}, \bar{d}, b], \quad \bar{y}=h(\bar{x}, b), \quad \bar{z}=h(\bar{x}, b)
$$

En una operación a lazo abierto (i.e., ecuación (2.7) con $u=\bar{u}$ ), el estado estacionario $\bar{x}$ puede ser estable o inestable (con un comportamiento de nodo, centro, punto silla o punto espiral), único o múltiple (Hamer et al., 1981; Guckenheimer y Holmes, 1983).

Se resolvió el sistema de ecuaciones algebraicas (2.13) para el sistema MMA-AC con la entrada nominal diseñada anteriormente (2.11). El reactor continuo de copolimerización presentó multiplicidad de estados estacionarios, de acuerdo con reportes de la literatura (Hamer et al., 1981; Schmidt et al., 1984; Teymour y Ray, 1989, 1992; Pinto y Ray, 1995a, 1995b). Encontrándose un punto de ignición (con conversión, temperatura y fracción de sólidos altos), un punto de extinción (con conversión, temperatura y fracción de sólidos bajos), y otro punto intermedio (ver Tabla 2.2, y notación al final de la tesis). Los dos puntos ignición y extinción son indeseables para operar el reactor; debido a que implican condiciones extremas: el punto de extinción corresponde a una baja conversión (5.8\%) y producción (26.2 $\mathrm{Kg} / \mathrm{h}$ ); el de ignición pese a tener una alta conversión, también tiene una alta 
temperatura de operación $\left(\bar{T}=372^{\circ} \mathrm{K}\right.$, siendo $353^{\circ} \mathrm{K}$ el limite de temperaturas moderadas para operar). Por lo que el estado de interés sería el punto intermedio.

Tabla 2.2 Estados estacionarios d el reactor continuo $\left(\mathrm{V}=2.0 \mathrm{~m}^{3}\right)$.

\begin{tabular}{|c|c|c|c|}
\hline$\vec{T}, \bar{Y}, \bar{Z}$ & Punto de lgnution & Punto intermedio & Punto de extinción \\
\hline $\bar{x}_{1}\left(\bar{m}_{1}\right)$ & 0.0031 & 0.0682 & 0.0818 \\
\hline $\bar{x}_{2}\left(\bar{m}_{2}\right)$ & 0.3231 & 0.4577 & 0.6627 \\
\hline $\bar{x}_{3}\left(\bar{p}_{1}\right)$ & 0.1055 & 0.0990 & 0.0313 \\
\hline $\bar{x}_{4}\left(\bar{p}_{2}\right)$ & 0.3304 & 0.2061 & 0.0088 \\
\hline $\bar{x}_{5}(\bar{i})$ & 0.00008 & 0.00083 & 0.0012 \\
\hline $\bar{x}_{6}(\bar{T})$ & 372.0 & 345.1 & 328.1 \\
\hline $\bar{x}_{7}(\bar{V})$ & 2.0 & 2.0 & 2.0 \\
\hline $\bar{x}_{8}\left(\bar{\mu}_{0}\right)$ & $4.540 \times 10^{-3}$ & $1.984 \times 10^{-3}$ & $2.954 \times 10^{-4}$ \\
\hline $\bar{x}_{9}\left(\bar{\mu}_{2}\right)$ & $8.942 \times 10^{7}$ & $9.933 \times 10^{7}$ & $1.139 \times 10^{7}$ \\
\hline $\bar{y}_{\rho}(\bar{\rho})$ & 1035.04 & 1001.76 & 936.56 \\
\hline $\bar{y}_{\eta}(\bar{\eta})$ & 1.4346 & 1.4229 & 1.3968 \\
\hline $\bar{y}_{T}(\bar{T})$ & 372.0 & 345.1 & 328.1 \\
\hline $\bar{y}_{V}(\bar{V})$ & 2.0 & 2.0 & 2.0 \\
\hline $\bar{z}_{s}\left(\bar{m}_{s}\right)$ & 0.4991 & 0.3606 & 0.0505 \\
\hline $\bar{z}_{c}(\bar{c})$ & 0.2126 & 0.2888 & 0.7491 \\
\hline $\bar{z}_{p}(\bar{p})$ & 0.6218 & 0.4439 & 0.0579 \\
\hline $\bar{z}_{M}(\bar{M})$ & 171,654 & 273,893 & 240,360 \\
\hline $\bar{z}_{R}(\bar{R})$ & 259.2 & 187.3 & 26.2 \\
\hline $\bar{\theta}_{\mathrm{r}}$ & 3.99 & 3.85 & 3.61 \\
\hline \multicolumn{4}{|c|}{$\begin{array}{l}\bar{u}_{1}\left(\bar{q}_{1}\right)=0.00111, \bar{u}_{2}\left(\bar{q}_{2}\right)=0.00623, \bar{u}_{3}\left(\bar{T}_{c}\right)=328, \bar{u}_{4}(\bar{q})=0.00864 \\
\bar{d}_{1}\left(\bar{m}_{1 e}\right)=1, \bar{d}_{2}\left(\bar{m}_{2 e}\right)=1, \bar{d}_{3}\left(\bar{p}_{1 e}\right)=0, \bar{d}_{4}\left(\bar{p}_{2 e}\right)=0, \bar{d}_{5}\left(\bar{T}_{1 e}\right)=315, \bar{d}_{6}\left(\bar{T}_{2 e}\right)=315, \bar{d}_{7}\left(\bar{T}_{s e}\right)=315, \\
\bar{d}_{8}\left(\bar{q}_{s}\right)=0.00199, \bar{d}_{9}\left(\bar{w}_{1 e}\right)=6.66 \times 10^{-5}\end{array}$} \\
\hline
\end{tabular}




\section{9}

Para determinar la estabilidad/inestabilidad de cada estado estacionario se empleo el primer método de estabilidad de Lyapunov (Slotine, 1991). Mientras que para verificar y determinar la región de multiplicidad de estados estacionarios se realizó un análisis de bifurcación (Guckenheimer y Holmes, 1983).

De acuerdo al primer método de Lyapunov, la estabilidad / inestabilidad (ver definiciones en el Apéndice $C$ ) de un estado estacionario, $\bar{x}$, se puede obtener de la estabilidad de la matriz jacobiana $f_{\mathrm{x}}=\partial f / \partial x$ evaluada en $(\bar{x}, \bar{u}, b)$. En el siguiente teorema se precisa la relación entre la estabilidad de un sistema no lineal y su correspondiente aproximación lineal (Slotine, 1991).

Teorema 1 (Método de linealización de Lyapunov). Si la aproximación lineal del sistema no lineal (2.7)

$$
\begin{gathered}
\dot{\tilde{x}}=A \tilde{x}+B \tilde{u} \\
\tilde{x}=x-\bar{x}, \quad \tilde{u}=u-\bar{u}, \quad A=f_{x}(\bar{x}, \bar{u}, b), \quad B=f_{u}(\bar{x}, \bar{u}, b)
\end{gathered}
$$

(i) Es estrictamente estable (i.e., si todos los valores propios de $A$ están estrictamente en el lado izquierdo del plano complejo), entonces el punto de equilibrio $\bar{x}$ del sistema no lineal (2.7) es asintóticamente estable.

(ii) Es inestable (i.e., si al menos uno de los valores propios de $A$ están estrictamente en el lado derecho del plano complejo), entonces el punto de equilibrio $\bar{x}$ del sistema no lineal (2.7) es inestable.

(iii) Es marginalmente estable (i.e., todos los valores propios de $A$ están en el lado izquierdo del plano complejo, pero al menos uno de ellos está sobre el eje imaginario), entonces no se puede concluir sobre la estabilidad partiendo de la aproximación lineal (el punto de equilibrio $\bar{x}$ del sistema no lineal (2.7) puede ser estable, asintóticamente estable, o inestable).

De acuerdo a este teorema se evaluaron los valores propios $\left(\lambda_{i}, 1 \leq i \leq 9\right)$ para los tres estados estacionarios de la Tabla 2.2, verificándose que los puntos extremos (extinción e ignición) son estables, mientras que el punto intermedio es inestable (como puede verse en la Tabla 2.3). 
Tabla 2.3 Estabilidad de los estad os estacionarios del reactor continuo $\left(\mathrm{V}=2.0 \mathrm{~m}^{3}\right)$.

\begin{tabular}{|c|c|c|c|}
\hline Valores propios & Estado de ignición & Estado intermedio & Estado de extinción \\
\hline$\lambda 1$ & -0.8 & -0.8 & -0.8 \\
\hline$\lambda_{2}$ & -0.00184 & -0.0014 & -0.0036 \\
\hline$\lambda_{3}$ & $-2.31 \times 10^{-4}$ & $-2.67 \times 10^{-4}$ & $-1.21 \times 10^{-4}$ \\
\hline$\lambda_{4}$ & $-1.48 \times 10^{-4}$ & $-3.11 \times 10^{-4}$ & $-8.25 \times 10^{-5}$ \\
\hline$\lambda_{5}$ & $-1.48 \times 10^{-4}$ & $-9.48 \times 10^{-5}$ & $-7.70 \times 10^{-5}$ \\
\hline$\lambda_{6}$ & $-6.97 \times 10^{-5}$ & $-9.48 \times 10^{-5}$ & $-7.70 \times 10^{-5}$ \\
\hline$\lambda_{7}$ & $-6.97 \times 10^{-5}$ & $-9.48 \times 10^{-5}$ & $-7.72 \times 10^{-5}$ \\
\hline$\lambda_{8}$ & $-6.97 \times 10^{-5}$ & $+1.81 \times 10^{-4}$ & $-7.72 \times 10^{-5}$ \\
\hline$\lambda_{9}$ & $-6.97 \times 10^{-5}$ & $+1.81 \times 10^{-4}$ & $-4.39 \times 10^{-5}$ \\
\hline & estable & Inestable & estable \\
\hline
\end{tabular}

El término "bifurcación" se ha utilizado para describir la "división" de las soluciones en equilibrio de una familia de ecuaciones diferenciales.

Sea el sistema de ecuaciones diferenciales

$$
\dot{x}=f_{\alpha}[x, u(t), b], \quad \alpha \in \mathrm{A} \subset R^{k}
$$

que depende del parámetro $\alpha$ ( $k$-dimensional), entonces las soluciones en equilibrio de (2.15) están dadas por las soluciones del sistema de ecuaciones algebraicas $f_{\alpha}[x, u(t), b]=0$. Como $\alpha$ varia, implica que este equilibrio está descrito por funciones suaves de $\alpha$ alejándose de aquellos puntos en los cuales la derivada del jacobiano de $f_{\alpha}[x, u(t), b]$ con respecto a $x, \partial f_{\alpha} / \partial x$, tiene un valor propio cero. La gráfica de cada una de estas funciones es una rama (bifurcación) de equilibrio de (2.15). En el equilibrio $\left(x_{0}, \alpha_{0}\right)$, donde $\partial f_{\alpha} / \partial x$ tiene un valor propio cero, varias ramas de equilibrio pueden colapsarse y se dice que $\left(x_{0}, \alpha_{0}\right)$ es un punto de bifurcación.

Para el caso particular de estudio se definió el parámetro de bifurcación $\alpha$ como la variación del tiempo de residencia, equivalente a cambiar los flujos de alimentación y de descarga; esto es

$$
\bar{\theta}_{r}=\alpha \theta_{r}
$$

donde $\bar{\theta}_{r}$ corresponde al tiempo de residencia nominal (Tabla 2.2), de tal forma que cuando $\alpha=1$ corresponde al punto de operación nominal diseñado anteriormente. 
En la Figura 2.2 se muestra el mapa de bifurcación para el reactor continuo, donde los puntos $(0, \bullet)$ localizados en $\alpha=1$ corresponden a los estados estacionarios nominales $\left(\mathrm{V}=2.0 \mathrm{~m}^{3}\right)$. En esta figura se observan tres regiones, tanto en la región 1 y 3 existe unicidad del estado estacionario; mientras que en la región dos existe multiplicidad de estados estacionarios (existencia de tres puntos, excepto en los limites de las regiones 1-2 y 2-3 donde sólo existen dos puntos). Por otra parte, se determinó (de acuerdo al Teorema 1) que las ramas superior e inferior corresponden a puntos estables, y la rama intermedia (entre los puntos de bifurcación) a puntos inestables. Interpretando fisicamente estos resultados se tiene que:

(a) La región 1 corresponde a tiempos de residencia altos (o flujos bajos), generando una aceleración de la reacción con fracción de sólidos, conversiones, viscosidades, y temperaturas altas; por lo que a estas condiciones no es recomendable operar el reactor.

(b) La región 3 corresponde a tiempos de residencia bajos (o flujos altos), provocando que el reactor actúe como un tanque de mezclado en el que la reacción se extingue; y consecuentemente tampoco es una región de interés.

(c) La región 2 presenta un caso drástico, por tener una combinación de los estados estacionarios (multiplicidad) de la región 1 y 3 , en conjunción con un punto crítico adicional. Este último por estar relacionado a condiciones de operación moderadas, seria el recomendable para operar el reactor; sin embargo, este punto es inestable y requiere de un diseño de control para poder alcanzarlo y estabilizarlo.

En resumen, para propósitos prácticos y de control el estado estacionario de interés es el punto inestable; puesto que este punto no tiene problemas de operación seguridad (como el caso del punto de ignición, con alta temperatura y fracción de sólidos) ni de producción (como el caso del punto de extinción, que tiene baja conversión y velocidad de producción).

Puesto que el reactor a lazo abierto no se puede operar en el punto inestable, entonces se diseñará una trayectoria de operación para propósitos de estimación a lazo abierto. Para este fin en la siguiente sección se definirá una trayectoria de operación, la cual deberá considerarse como una condición severa ya que se elegirá como punto inicial de operación al estado estacionario (inestable) intermedio y como punto final de operación al estado estacionario (estable) de ignición. 


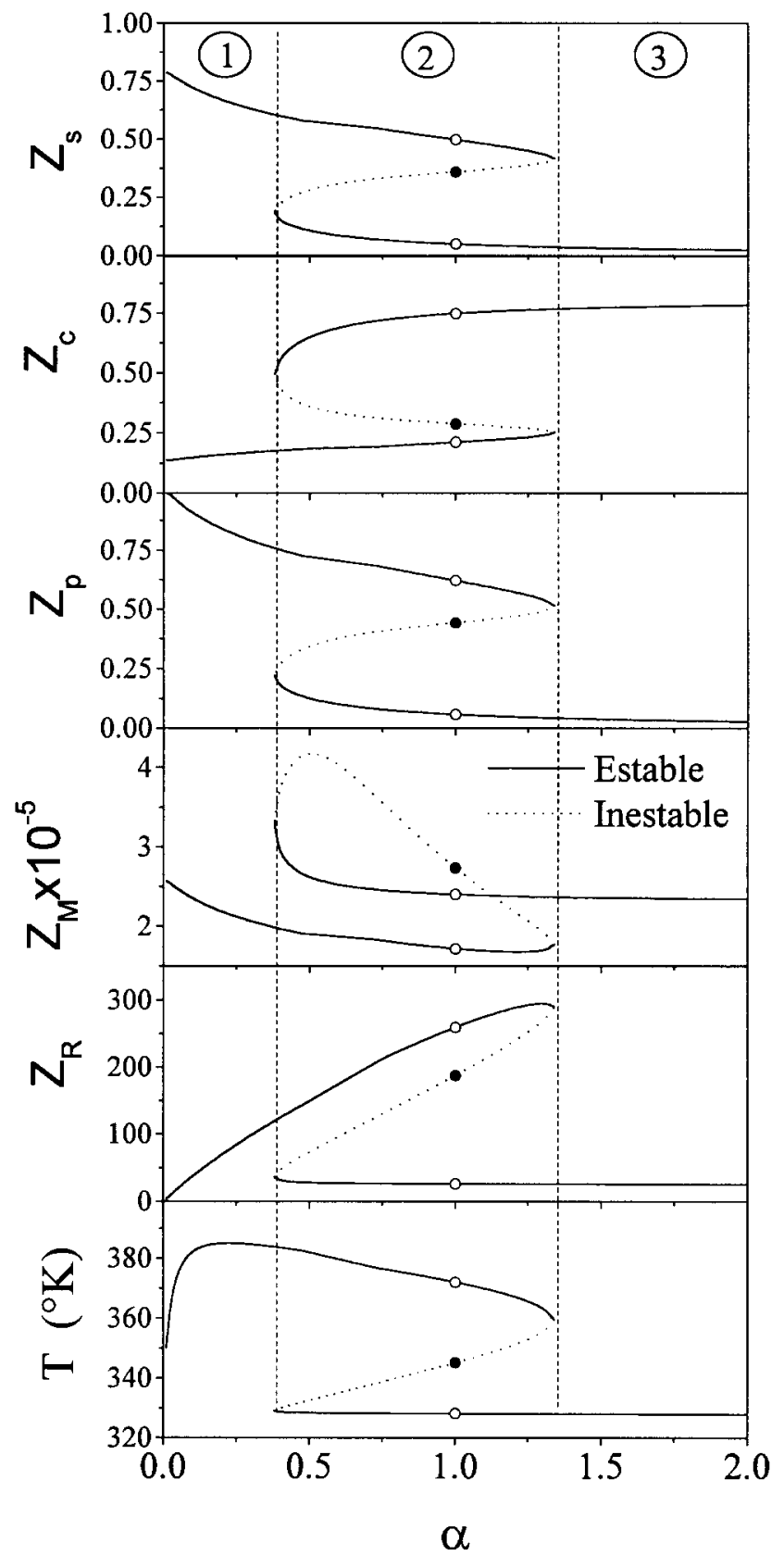

Figura 2.2 Mapa de bifurcación para el reactor de copolimerización $\left(\alpha=\bar{\theta}_{r} / \theta_{r}\right)$. 


\subsubsection{El reactor no autónomo}

Para la trayectoria de operación se consideraron dos volúmenes: un volumen inicial de $1.5 \mathrm{~m}^{3}$, y un volumen final de $2.0 \mathrm{~m}^{3}$. Para cada volumen, el reactor continuo tiene (al menos) tres estados estacionarios, dos estables (alta y baja conversión), y uno inestable (conversión intermedia). En la Figura 2.3 se muestran la proyección de la trayectoria $x(t)$ en el espacio $P: z_{\mathrm{s}}$ (fracción de sólidos) - $T$ (temperatura) - $z_{\mathrm{M}}$ (peso molecular promedio-peso), de los tres estados estacionarios [ $\square \mathrm{I}\left(\mathrm{S}_{1}\right), \square \mathrm{I}(\mathrm{U}), \square \mathrm{I}\left(\mathrm{S}_{2}\right)$ ] del volumen inicial $\left(\mathrm{V}=1.5 \mathrm{~m}^{3}\right)$, asi como los tres estados estacionarios $\left[\mathrm{OF}\left(\mathrm{S}_{1}\right)\right.$, - $\mathrm{F}(\mathrm{U}), \mathrm{OF}\left(\mathrm{S}_{2}\right)$ correspondiente al volumen final $\left(\mathrm{V}=2.0 \mathrm{~m}^{3}\right)$.

La trayectoria de operación es como sigue: Inicialmente, el reactor se encuentra en el estado estacionario inestable [ I (U) en Tabla 2.4 y Figura 2.3] del volumen de operación de $1.5 \mathrm{~m}^{3}$. Luego es sometida a entradas exógenas variantes en tiempo como se muestra en la Figura 2.4a, y al cambio de volumen de acuerdo a:

$$
q=q_{1}+q_{2}+q_{s}-\left[\varepsilon_{1} r_{1} M_{1}^{o}+\varepsilon_{2} r_{2} M_{2}^{o}\right] \mathrm{V}-\frac{4}{5}[V-\bar{V}(t)], \quad \bar{V}(t)=1.5+0.5\left(1-e^{-\frac{4 t}{600}}\right)
$$

tal que, el reactor alcanza asintóticamente (con un $1.987 \%$ - tiempo de asentamiento de $600 \mathrm{~min}$ ) el estado estacionario estable de alta conversión $\left[\mathrm{OF}\left(\mathrm{S}_{2}\right)\right.$ en Tabla 2.4 y

Figura 2.3] del volumen de operación de $2.0 \mathrm{~m}^{3}$.

En la Figura 2.3 se muestra la curva correspondiente a la proyección de la trayectoria nominal del reactor en el espacio $P\left(z_{\mathrm{s}}-T-z_{\mathrm{M}}\right)$. En la Figura 2.4 se presentan las correspondientes evoluciones en tiempo del reactor (_ $\ldots-\ldots$ ), la cual muestra que la dinámica del reactor está altamente excitada, experimentado cambios considerables y abruptos debido a la competencia de tendencias entre extinción e ignición. Después de pasar a través de la región difícil (i.e., región con fuerte efecto gel y limitación en la remoción de calor, asi como fracción de sólidos, conversión y temperaturas altas), el reactor alcanza asintóticamente su estado estacionario final de alta conversión. Esta trayectoria nominal del reactor evoluciona a través de un conjunto amplio de intervalos de operación: la temperatura entre $[350,425]^{\circ} \mathrm{K}$, la fracción de sólidos entre $[0.4,0.55]$, la composición instantánea entre $[0.15,0.30]$, la conversión entre [0.5, 0.7], el peso molecular promedio-peso entre [160 000, 210000$] \mathrm{Kg} / \mathrm{Kmol}, \mathrm{y}$ la velocidad de producción entre [200, 500] $\mathrm{Kg} / \mathrm{h}$. 
Tabla 2.4 Estados y salidas (iniciales/finales) de la trayectoria de operación y perturbada, y valores de las entradas exógenas constantes y variantes en tiempo.

\begin{tabular}{|c|c|c|c|}
\hline \multirow{2}{*}{$x, y, z$} & \multicolumn{2}{|c|}{ Trayectoria de operación, x(t) } & \multirow{2}{*}{$\begin{array}{l}\text { Trayec perturbada } x(t) \\
\text { Estado inicial }(*)\end{array}$} \\
\hline & 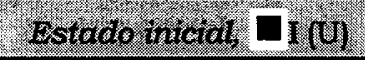 & Estado finat, $\mathrm{OF}\left(\mathrm{s}_{2}\right)$ & \\
\hline$x_{1}\left(m_{1}\right)$ & 0.0050 & 0.0031 & 0.0070 \\
\hline$x_{2}\left(m_{2}\right)$ & 0.4067 & 0.3231 & 0.3030 \\
\hline$x_{3}\left(p_{1}\right)$ & 0.1018 & 0.1055 & 0.0800 \\
\hline$x_{4}\left(p_{2}\right)$ & 0.2533 & 0.3304 & 0.3500 \\
\hline$x_{5}(i)$ & 0.0004 & 0.00008 & 0.0005 \\
\hline$x_{6}(T)$ & 358.0 & 372.0 & 370.0 \\
\hline$x_{7}(V)$ & 1.5 & 2.0 & 1.7 \\
\hline$x_{8}\left(\mu_{0}\right)$ & $3.432 \times 10^{-3}$ & $4.540 \times 10^{-3}$ & $5.0 \times 10^{-3}$ \\
\hline$x_{9}\left(\mu_{2}\right)$ & $7.814 \times 10^{7}$ & $8.942 \times 10^{7}$ & $7.5 \times 10^{7}$ \\
\hline$y_{\rho}(\rho)$ & 1014.46 & 1035.04 & 1033.40 \\
\hline$y_{\eta}(\eta)$ & 1.4275 & 1.4346 & 1.4323 \\
\hline$y_{T}(T)$ & 358.0 & 372.0 & 370.0 \\
\hline$y_{V}(V)$ & 1.5 & 2.0 & 1.7 \\
\hline$z_{s}\left(m_{s}\right)$ & 0.4146 & 0.4991 & 0.4936 \\
\hline$z_{c}(c)$ & 0.2537 & 0.2126 & 0.1620 \\
\hline$z_{p}(p)$ & 0.5132 & 0.6218 & 0.6330 \\
\hline$z_{M}(M)$ & 184,694 & 171,654 & 150,000 \\
\hline$z_{R}(R)$ & 215.3 & 259.2 & 380.0 \\
\hline $\bar{\theta}_{\mathrm{r}}$ & 2.93 & 3.99 & -- \\
\hline \multicolumn{4}{|c|}{$\begin{array}{l}\text { Entradas constantes : } \bar{u}_{1}\left(\bar{q}_{1}\right)=0.00111, \bar{u}_{2}\left(\bar{q}_{2}\right)=0.00623, \bar{u}_{3}\left(\bar{T}_{c}\right)=328 \\
\bar{d}_{2}\left(\bar{m}_{2 e}\right)=1, \bar{d}_{3}\left(\bar{p}_{1 e}\right)=0, \bar{d}_{4}\left(\bar{p}_{2 e}\right)=0, \bar{d}_{8}\left(\bar{q}_{s}\right)=0.00199, \bar{d}_{9}\left(\bar{w}_{l e}\right)=6.66 \times 10^{-5}\end{array}$} \\
\hline \multicolumn{4}{|c|}{$\begin{array}{l}\text { Valores iniciales (finales) de las entradas variantes en tiempo: } \bar{u}_{4}(\bar{q})=0.00853(0.00836) \\
\bar{d}_{1}\left(\bar{m}_{1 e}\right)=1(1), \bar{d}_{5}\left(\bar{T}_{1 e}\right)=\bar{d}_{6}\left(\bar{T}_{2 e}\right)=\bar{d}_{7}\left(\bar{T}_{s e}\right)=315(315)\end{array}$} \\
\hline
\end{tabular}




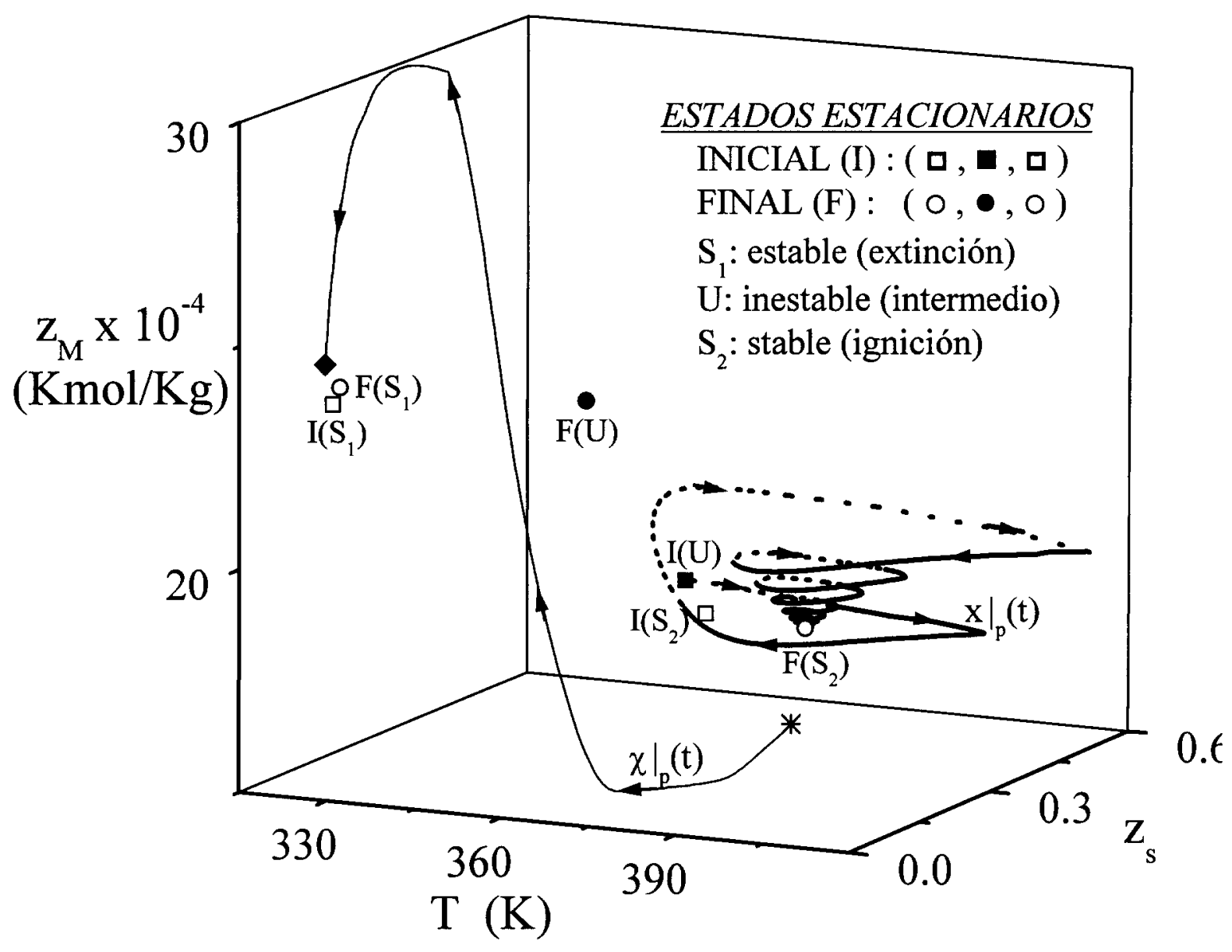

Figura 2.3 Proyección de la trayectoria $x(t)$ en el espacio $P: z_{\mathrm{s}}-T-z_{\mathrm{M}}$. 
(a) ENTRADAS EXOGENAS
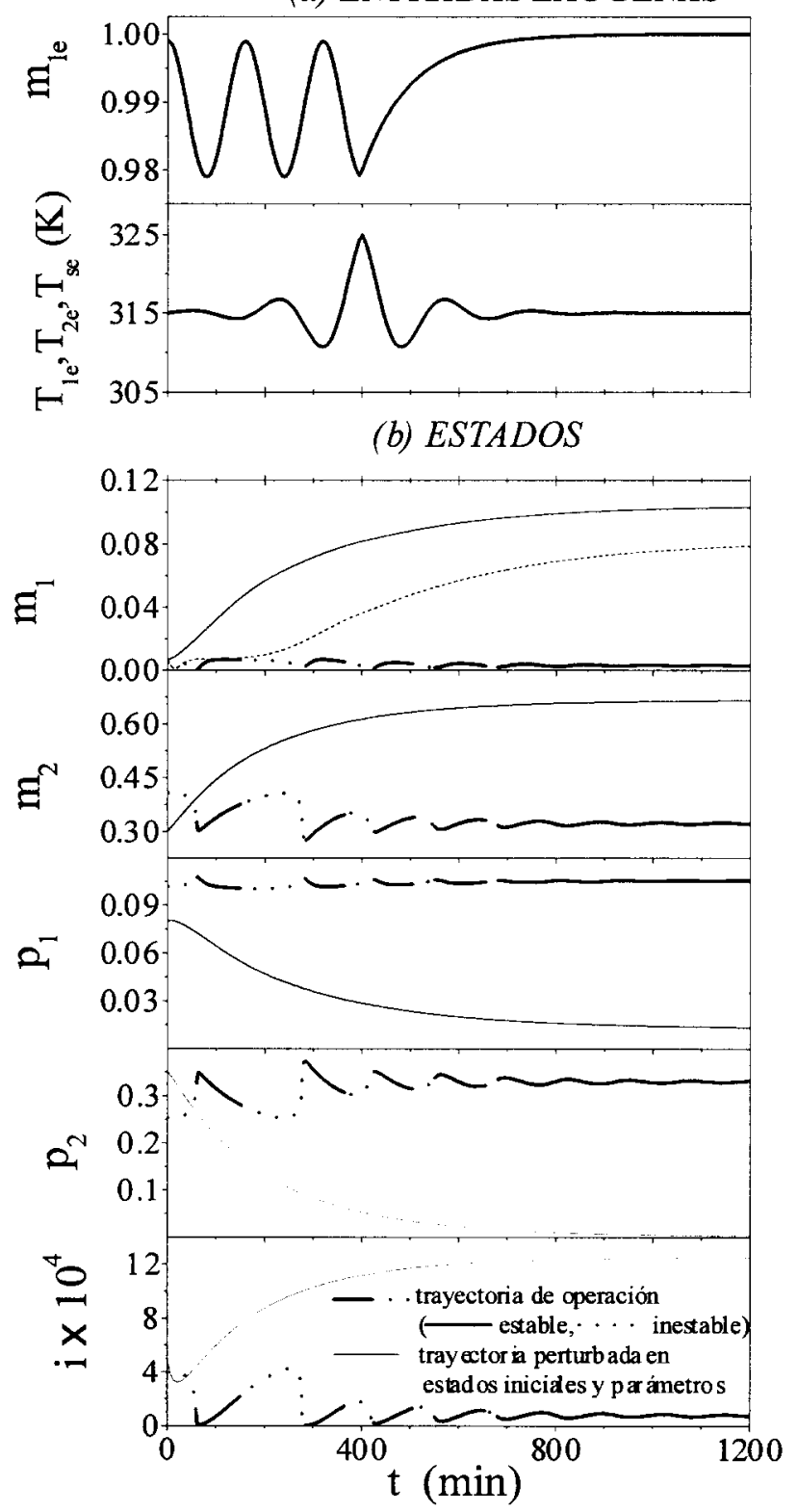

Figura 2.4 (a) Entradas exógenas y (b) estados de las trayectorias nominal y perturbada 
(c) MEDICIONES

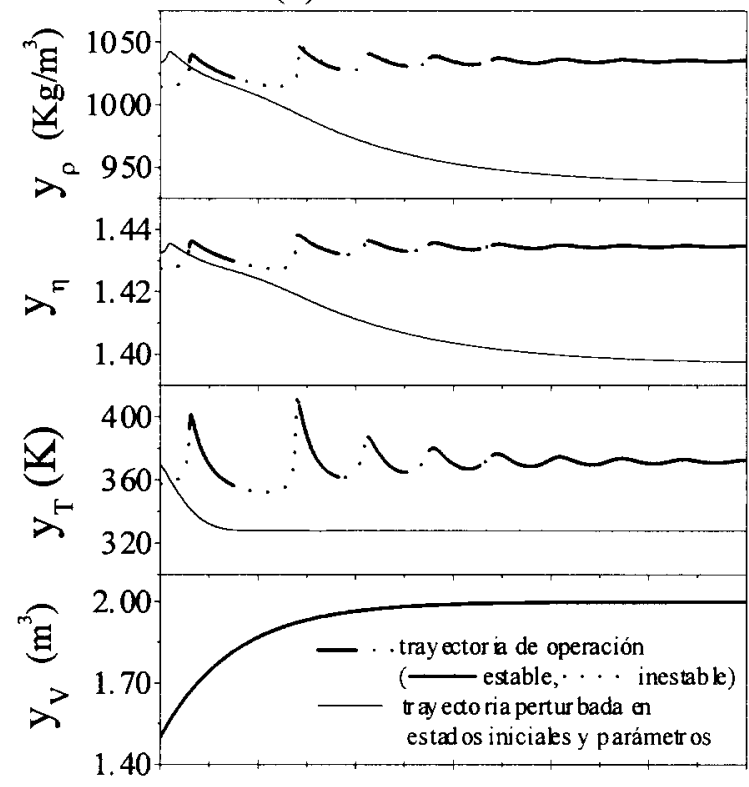

(d) SALIDA z

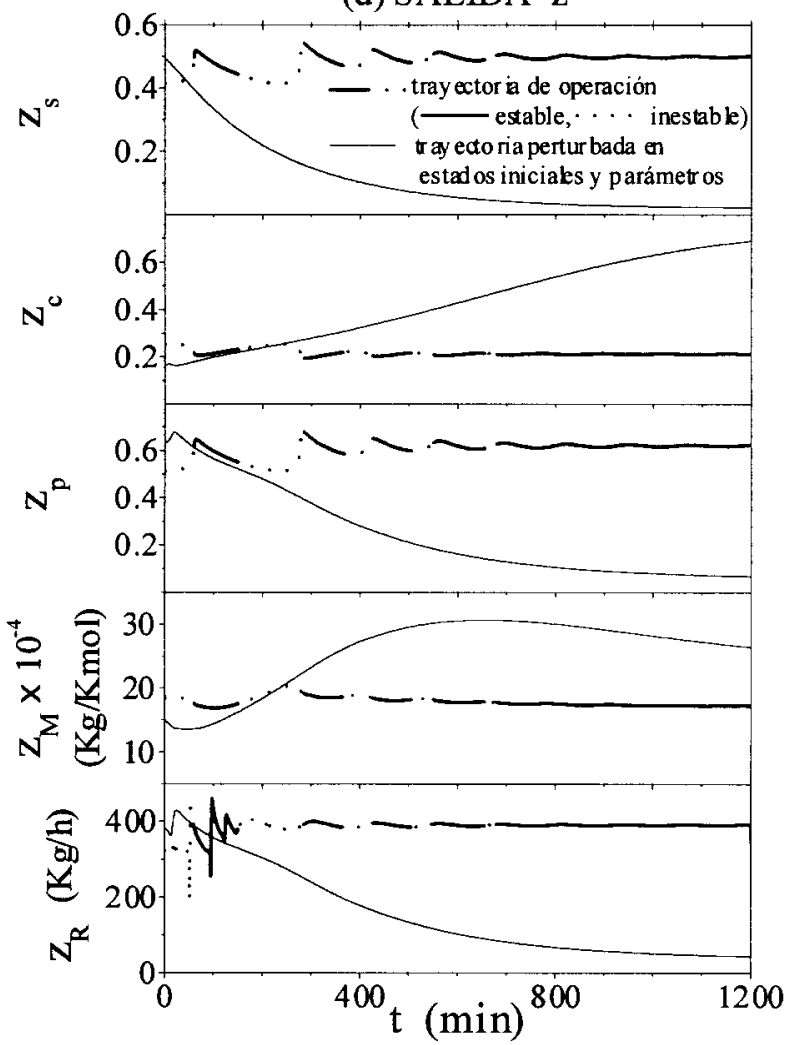

Figura 2.4 (c) Salidas medidas y (d) salidas de interés de las trayectorias nominal y perturbada 
La determinación de la estabilidad/inestabilidad de una trayectoria se puede realizar mediante varios procedimientos. Aqui se emplearon dos métodos, el primero consiste en perturbar la trayectoria nominal en las condiciones iniciales de los estados $\chi_{0}$ en lugar de $x_{\mathrm{o}}$ ) y los parámetros del modelo ( $\beta$ en lugar de b), y mediante simulaciones numéricas verificar si es estable en el sentido de Lyapunov (esto es, si la trayectoria perturbada se mantiene arbitrariamente cerca de la trayectoria nominal entonces es estable).

El segundo método empleado para verificar la estabilidad fue el análisis de los valores propios del sistema lineal aproximado. En el caso de un reactor autônomo la estabilidad puede verificarse de acuerdo al primer método de Lyapunov. Sin embargo, la estabilidad de la trayectoria $x(t)$ de un sistema no autónomo, no necesariamente se obtiene a partir de la matriz jacobiana $f_{\mathrm{x}}$ a lo largo de la trayectoria, en cualquier tiempo $t \geq 0$. Una condición suficiente para la estabilidad de la trayectoria $x(t)(2.9)$ del sistema no autónomos (2.7), derivada del segundo método de Lyapunov, es la siguiente (Slotine y Li, 1991): La matriz simétrica $F(t)=f_{x}(t)+$ $f_{\mathrm{x}}{ }^{\prime}(t)$ ] debe ser estable para todo $t \geq 0$, si la función $l_{\mathrm{s}}(t)$ de margen de estabilidad (o de decaimiento exponencial) dada por

$$
l_{s}(t)=\frac{1}{2} \min _{0 \leq i \leq n}\left\{-\lambda_{1}(t), \ldots,-\lambda_{n}(t)\right\}>0
$$

es estrictamente positiva. Siendo $\lambda_{1}(t), \ldots, \lambda_{\mathrm{n}}(t)$ son los valores propios (reales) de la función $F(t)$.

En la Figura 2.4 se presenta la trayectoria perturbada del reactor $\chi(t)$ con errores en: las condiciones iniciales de los estados (ver Tabla 2.4, y punto * en Figura 2.3), y en los parámetros del modelo listados en la Tabla 2.5). Es importante señalar que, estos valores de los estados y parámetros del modelo serán usados para la implementación del estimador y controlador en los siguientes capítulos, por lo que la trayectoria perturbada nos permitirá obtener las características y el comportamiento limite alcanzable.

Tabla 2.5 Valores de los parámetros del modelo para la trayectoria nominal y perturbada, respectivamente.

\begin{tabular}{|c|c|c|}
\hline Parametro & Valor nominal (b) & Valor perturbado (B) \\
\hline$E_{p 1}$ & 24,200 & 23,230 \\
\hline$E_{p 2}$ & 18,000 & 17,280 \\
\hline$a_{h}$ & 0.740 & 0.592 \\
\hline$\varepsilon_{1}$ & 0.1880 & 0.0 \\
\hline$\varepsilon_{2}$ & 0.2168 & 0.0 \\
\hline
\end{tabular}




\section{9}

Los parámetros del modelo listados en la Tabla 2.5) corresponden a las energias de activación $\left(E_{\mathrm{p} 1}\right.$ y $\left.E_{\mathrm{p} 2}\right)$ de las reacciones de propagación, la constante (adimensional) de proporcionalidad $\left(a_{\mathrm{h}}\right)$ de la expresión del coeficiente de transferencia de calor tipoNusselt (Bondy y Lippa, 1983), y los factores de contracción de volumen $\left(\varepsilon_{1}, \varepsilon_{2}\right)$ de los monómeros; todos ellos dentro del mapa no lineal $f[x, u(t), b]$ del reactor (2.7). Los errores en los parámetros implican una conjunción de factores desfavorables que significan una sobreestimación del $25 \%$ en las velocidades de polimerización y de generación de calor, y un $20 \%$ de subestimación en la velocidad de remoción de calor.

En general, con pequeños errores en las condiciones iniciales, las trayectorias perturbadas alcanzan el estado estacionario de extinción $\left[\mathrm{OF}\left(\mathrm{S}_{1}\right)\right.$ en la Figura 2.3] o bien el punto de ignición [ $\mathrm{OF}\left(\mathrm{S}_{2}\right)$ en la Figura 2.3] del volumen de operación de 2.0 $\mathrm{m}^{3}$. Esto indica que la trayectoria nominal es inestable. Con los errores iniciales (* en Figura 2.3) y los errores en los parámetros del modelo (dados en la Tabla 2.5) para la trayectoria perturbada (__ ), en la Figura 2.3 se muestra la proyección en el espacio $P\left(z_{\mathrm{s}}-T-z_{\mathrm{M}}\right)$, observándose que en lugar de alcanzar el estado estacionario final de alta conversión $\left[\mathrm{OF}\left(\mathrm{S}_{2}\right)\right]$ con oscilaciones amortiguadas, la trayectoria perturbada alcanza un estado estacionario (*) alrededor del estado de baja conversión $\left[\mathrm{OF}\left(\mathrm{S}_{1}\right)\right]$ del volumen de $2.0 \mathrm{~m}^{3}$, con una respuesta subamortiguada. Esto mismo se observa en la Figura 2.4, donde se muestran la evolución en tiempo de la trayectoria perturbada.

Por otra parte, se empleo el método de análisis de valores propios con base en la desigualdad definida en (2.17). De acuerdo a este análisis, en la proyección en el espacio $P$ (Figura 2.3) y en las trayectoria dinámicas (Figura 2.4) se indican secciones continuas y discontinuas denotando que la aproximación lineal del reactor es estable e inestable, respectivamente. Con lo cual se concluye que la trayectoria es inestable.

La inestabilidad de la aproximación lineal del reactor y divergencia de la trayectoria perturbada corroboran la inestabilidad de la trayectoria nominal $x(t)$, la cual debe verse como el caso de un estimador/controlador geométrico no lineal con ganancia cero. Además exhibe cual debe ser el comportamiento a alcanzar con un estimador robusto: hacer converger la trayectoria perturbada $\chi(t)$ hacia la trayectoria actual del reactor $x(t)$. 


\section{Capítulo 3}

\section{ESTIMACIÓN DE ESTADOS NO LINEAL}

Como un preámbulo al estudio del problema de estimación del reactor, en este capítulo se presenta un estimador para sistemas dinámicos no lineales (no autónomos) en general. Se establece la propiedad de estimabilidad de plantas no lineales, asi como la construcción, el criterio de convergencia, y el esquema de sintonización del estimador geométrico no lineal propuesto. Proponiéndose dos diseños para el estimador, uno tipo $P$ (proporcional) y un segundo tipo PI (proporcional-integral). Este último con la finalidad de compensar los errores de modelado y atenuar el ruido en las mediciones. Posteriormente se establecen los grados de libertad del esquema de estimación, que son primordialmente: la estructura de estimabilidad y la técnica de sintonización. Por último, se discuten algunos aspectos que deben tomarse en cuenta para el diseño e implementación del estimador. 


\subsection{E1 problema de estim ación}

Para el estudio de estimación a lazo abierto, consideraremos un solo vector de entradas $(u)$ que engloba a las entradas de control y las entradas exógenas. Y consideremos la planta no lineal no autónoma (esto es, con entradas exógenas variantes en tiempo) MEMS (múltiples entradas - múltiples salidas)

$$
\dot{x}=f[x, u(t), b], \quad y=h(x, b), \quad z=h_{z}(x, b) ; \quad x\left(t_{o}\right)=x_{o}
$$

con $n$ estados $(x), m$ salidas medibles $(y), m_{\mathrm{e}}$ salidas de interés $(z), p$ entradas $(u)$, y $n_{\mathrm{p}}$ parámetros del modelo $(b)$. Las entradas $u_{1}(t), \ldots, u_{\mathrm{p}}(t)$ son funciones del tiempo continuas por tramos; y los mapas $f, h$ y $h_{z}$ son suficientemente suaves (diferenciables) alrededor de la evolución $\mathrm{E}(t)$

$$
E(t)=\{x(t), u(t), y(t)\}
$$

Donde la trayectoria

$$
x(t)=\theta_{x}\left[t, t_{o}, x_{o}, u(t), b\right]
$$

(posiblemente inestable) está determinada únicamente por el conjunto de datos $\left\{x_{o}\right.$, $u(t)$, b (estados - entradas - parámetros iniciales). La trayectoria $x(t)$ del sistema (3.1) describe la evolución de los procesos por lotes y semicontinuos, asi como arranques, paros, y cambios de procesos continuos. Un proceso continuo es un caso particular (autónomo) de la planta (3.1), alrededor de una trayectoria invariante en tiempo $x$ (esto es, un estado estacionario o punto de equilibrio). Mientras que la estabilidad, observabilidad y controlabilidad de un sistema autónomo son propiedades que se cumplen para un estado estacionario, en sistemas no autónomos estas propiedades se cumplen para una evolución.

Los conceptos generales de estabilidad en el sentido de Lyapunov, estabilidad asintótica, y estabilidad exponencial se han tomado de la literatura (Slotine, 1991; Kwakernaak y Sivan, 1972) e incluido en el Apéndice C. A continuación se reseñan los conceptos de estabilidad nominal y estabilidad robusta, los cuales son claves para estudiar y diseñar el estimador.

Las siguientes definiciones de normas de vectores se emplearán en este capitulo. $\|x\|$ es la norma euclediana del vector $x$ en un espacio $n$-dimensional, y $\|u(t)\|^{s}$ es la norma de un vector cambiante en tiempo:

$$
\|x\|=\left(x_{1}^{p}+\ldots+x_{n}^{p}\right)^{1 / p}, \quad p>1 ; \quad\|u(t)\|^{s}=\sup _{t}\|u(t)\|
$$


La trayectoria $x(t)(3.3)$ es $N$ (nominalmente)-estable si las trayectorias perturbadas (en el estado inicial)

$$
\chi(t)=\theta_{x}\left[t, t_{o}, \chi_{o}, u(t), b\right], \quad\left\|\chi_{o}-x_{o}\right\| \leq \varepsilon_{o}
$$

pueden permanecer arbitrariamente cerca de la trayectoria no perturbada $x(t)$, empezando suficientemente cerca de ella.

La trayectoria $x(t)$ (3.3) es $R$ (robustamente)-estable si las trayectorias perturbadas (en el estado - entrada - parámetro inicial)

$$
\chi(t)=\theta_{x}\left[t, t_{o}, \chi_{o}, \mathrm{v}(t), \beta\right], \quad\left\|\chi_{o}-x_{o}\right\| \leq \varepsilon_{o}, \quad\|\mathrm{v}(t)-u(t)\| \leq \varepsilon_{u}, \quad\|\beta-b\| \leq \varepsilon_{b}
$$

pueden permanecer arbitrariamente cerca de la trayectoria no perturbada $x(t)$, empezando suficientemente cerca de ella y haciendo los errores de las entradas exógenas y parámetros suficientemente pequeños. A continuación, las versiones exponenciales de estas nociones de estabilidad se definen técnicamente.

Definición 1 (Estabilidad exponencial/robustamente exponencial). La trayectoria de estados $x(t) \quad(3.3)$ es E(exponencialmente)-estable si existen dos números estrictamente positivos $\left(a_{x}, \lambda_{x}\right)$ tal que, en alguna vecindad de la evolución $\mathrm{E}(t)$ (3.2), las trayectorias perturbadas $\chi(t)(3.4)$ convergen como sigue

$$
\|\chi(t)-x(t)\| \leq a_{x} e^{-\lambda_{x}\left(t-t_{o}\right)}\left\|\chi_{o}-x_{o}\right\|
$$

La trayectoria $x(t)$ es $R E$ (robustamente exponencialmente)-estable si existen cuatro constantes estrictamente positivas $\left(a_{\mathrm{x}}, \lambda_{\mathrm{x}}, b_{\mathrm{u}}, a_{\mathrm{b}}\right)$ tal que, las trayectorias perturbadas $\chi(t)(3.5)$ convergen como sigue

$$
\|\chi(t)-x(t)\| \leq a_{x} e^{-\lambda_{x}\left(t-t_{o}\right)}\left\|\chi_{o}-x_{o}\right\|+b_{u}\|\mathrm{v}(t)-u(t)\|^{s}+b_{b}\|\beta-b\|, \quad\|\mathrm{v}(t)-u(t)\|^{s}={ }_{t}^{s u p}\|\mathrm{v}(t)-u(t)\|
$$

La evolución $\mathrm{E}(t)(3.2)$ es $\mathrm{E} / \mathrm{RE}$-estable si $x(t)$ es $\mathrm{E} / \mathrm{RE}$ estable.

Observactón 3.1. La Definición 1 significa que, si la trayectoria $x(t)$ es perturbada con pequeños errores en las condiciones iniciales de los estados $\left(\chi_{0}\right.$ en lugar de $\left.x_{0}\right)$ y si la trayectoria perturbada $(\chi(t))$ se mantiene arbitrariamente cerca de la trayectoria $x(t)$, entonces $x(t)$ es E-estable. Más aún, si la trayectoria $x(t)$ es perturbada tanto en las condiciones iniciales de los estados $\left(\chi_{0}\right.$ en lugar de $\left.x_{0}\right)$ como en la evolución de las entradas $(u(t)$ en lugar de $u(t))$, y si la trayectoria perturbada $(\chi(t))$ se mantiene arbitrariamente cerca de la trayectoria $x(t)$, entonces $x(t)$ es RE-estable. 
El problema de estimación consiste en diseñar un procesador dinámico de datos

$$
\dot{\hat{x}}_{e}=f_{e}\left[\hat{x}_{e}, u(t), y(t), \beta\right], \quad \hat{x}=h_{e}\left(\hat{x}_{e}\right), \quad \hat{y}=h(\hat{x}, \beta), \quad \hat{z}=h_{z}(\hat{x}, \beta) ; \quad \hat{x}_{e}=\left(\hat{x}^{\prime}, \hat{x}_{m}{ }^{\prime}\right)^{\prime}
$$

que, construido con un vector de parámetros aproximado $\beta$ y accionado por las mediciones $u(t)$ y $y(t)$, genera:

(i) una trayectoria $\hat{x}(t)$ que RE-converge a la trayectoria $x(t)$ de la planta,

(ii) una trayectoria de salida $\hat{y}(t)$ que E-converge sin sesgo a la salida medida $y(t)$, con dinámica c(cuasi)-LNPA(lineal no-interactiva de polos asignables), y

(iii) un trayectoria de salida $\hat{z}(t)$ que RE-converge a la salida actual $z(t)$.

El estimador de estados $\hat{x}_{\mathrm{e}}$ incluye el estimado de estados actual $\hat{x}$ asi como un estado aumentado $\hat{x}_{\mathrm{m}}$, para permitir que se reproduzcan asintóticamente las entradas $(\mathrm{u})$-salidas $(\mathrm{y})$ de la planta (mediante la compensación de las perturbaciones persistentes causadas por los errores de modelado).

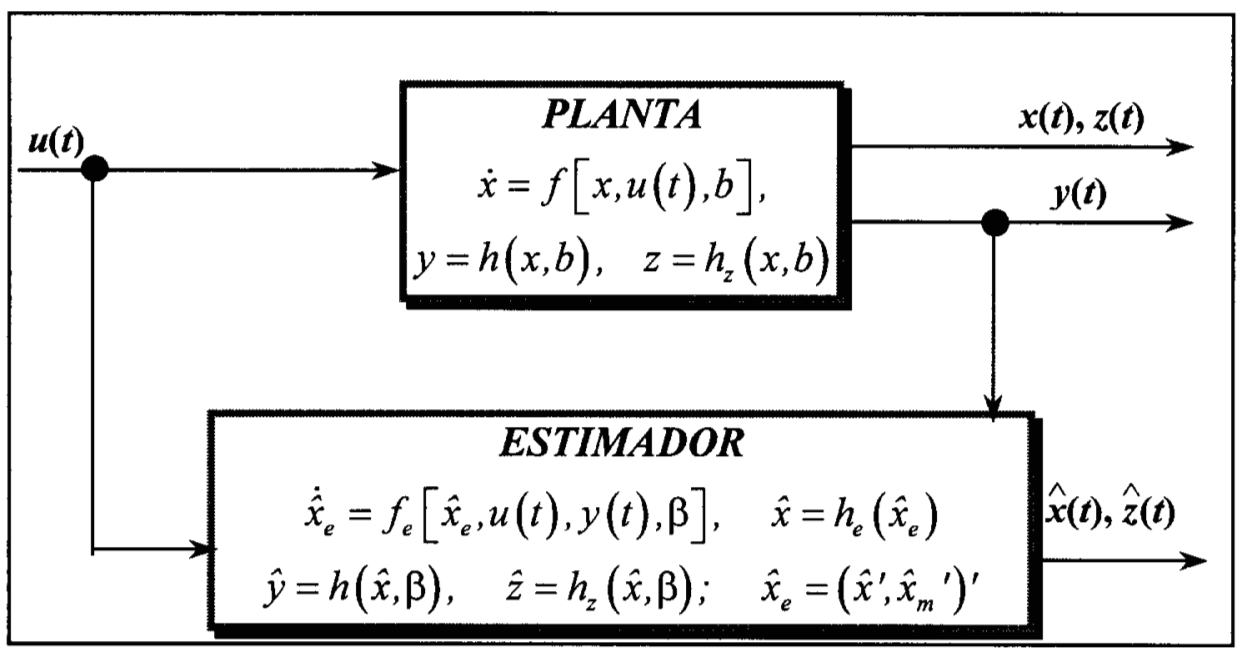

Figura 3.1 Diagrama de bloques del estimador de estados 


\subsection{Propiedad de Estima bilidad}

Para presentar e interpretar la noción de estimabilidad que define la teoría de estimación geométrica no lineal, consideremos un problema auxiliar (Alvarez, 2000): la reconstrucción en linea de la trayectoria de estados $x(t)$ (3.3) cuando los parámetros del modelo $b$, las señales medidas $u(t)$ y $y(t)$, y sus derivadas temporales son conocidos. Para este propósito, tomemos derivadas temporales sucesivas del mapa de salida $y_{\mathrm{i}}=h_{\mathrm{i}}(x, b)$ de la ecuación (3.1), hasta el orden $\kappa_{\mathrm{i}}$, obteniendo el conjunto de $(\kappa+m)$ ecuaciones (algebraicas, variantes en tiempo):

$$
\begin{gathered}
y(t)=\phi_{I}[x, \mathcal{U}(t), b], \quad \operatorname{dim}(y)=\kappa_{1}+\ldots+\kappa_{m} \\
v(t)=\varphi[x, \mathcal{U}(t), b], \quad \operatorname{dim}(v)=m
\end{gathered}
$$

donde $y, u$, y $v$ son vectores obtenidos a partir de las señales medidas $\mathrm{y}$ de sus derivadas temporales,

$$
\begin{array}{ll}
y=\left[y_{1}, \ldots, y_{1}^{\left(\kappa_{1}-1\right)} ; \ldots ; y_{m}, \ldots, y_{m}^{\left(\kappa_{m}-1\right)}\right]^{\prime}, & v=\left[y_{1}^{\left(\kappa_{1}\right)}, \ldots, y_{m}^{\left(\kappa_{m}\right)}\right]^{\prime}, \quad \kappa_{1}+\ldots+\kappa_{m}=\kappa \leq n \\
\mathcal{U}=\left[u_{1}, \ldots, u_{1}^{\left(v_{1}-1\right)} ; \ldots ; u_{p}, \ldots, u_{p}^{\left(v_{p}-1\right)}\right]^{\prime}, & v_{1}+\ldots+v_{p}=v
\end{array}
$$

$\phi_{\mathrm{I}}$ y $\varphi$ son mapas no lineales dados por:

$$
\begin{gathered}
\phi_{I}(x, \mathcal{U}, b)=\left[h_{1}, \ldots, L_{f}^{\mathrm{K}_{1}-1} h_{1} ; \ldots, h_{m}, \ldots, L_{f}^{\mathrm{K}_{m}-1} h_{m}\right]^{\prime} \\
\varphi(x, \mathcal{U}, v, b)=\left[L_{f}^{\mathrm{K}_{1}} h_{1}, \ldots, L_{f}^{\mathrm{K}_{m}} h_{m}\right]^{\prime}
\end{gathered}
$$

Donde $L_{f}^{i} h$ es la i-êsima derivada direccional recursiva del campo escalar variante en tiempo $\alpha(x, t)$ a lo largo del campo vectorial $f(x, t)$ :

$$
\begin{gathered}
L_{f}^{i+1} \alpha=L_{f}\left(L_{f}^{i} \alpha\right), \quad i \geq 1 ; \quad L_{f}^{0} \alpha=\alpha ; \\
L_{f} \alpha=\alpha_{x} f+\alpha_{t}, \quad \alpha_{x}=\frac{\partial \alpha}{\partial x}, \quad \alpha_{t}=\frac{\partial \alpha}{\partial t}
\end{gathered}
$$

Observación 3.2. Los indices de observabilidad $\left(\kappa_{1}, \ldots, \kappa_{m}\right)$ indican el número de derivadas temporales que se emplearán $\left(\kappa_{\mathrm{i}}-1\right.$ derivadas de la salida $\left.y_{\mathrm{i}}\right)$ para la construcción del estimador. El indice de observabilidad global $\kappa=\kappa_{1}+\ldots+\kappa_{\mathrm{m}}$ determina a su vez el número de estados observables, siendo por lo tanto ( $n-\kappa)$ el número de estados no observables. Por otra parte, el conjunto $k=\left(\kappa_{1}, \ldots, \kappa_{\mathrm{m}}\right)$ puede definirse de manera combinatorial, tal que cumplan la condición: $\kappa_{1}+\ldots+\kappa_{\mathrm{m}} \leq n$. Por ejemplo, si $n=3$ (3 estados) y $m=2$ (2 mediciones), entonces el conjunto $k=\left(\kappa_{1}, \kappa_{2}\right)$ puede ser definido como: $(1,1),(2,1),(1,2)$. La elección del conjunto $k$ y su influencia en el desempeño de estimador se establecerá en las secciones 3.6 y 3.7 . 
Consideremos el conjunto de $\kappa$-ecuaciones (3.7) que admite una solución única

$$
x_{I}=\phi_{I}^{-1}\left[x_{I I}, \mathcal{U}(t), y(t), b\right], \quad \operatorname{dim}\left(x_{I}, x_{I I}\right)=(\kappa, n-\kappa)
$$

para $\kappa$ de los estados del vector $x$ ( $n$-dimensional), o equivalentemente, que el mapa $\phi_{I}$ es $x_{\mathrm{I}}$-invertible (esto es, invertible para $x_{\mathrm{I}}$; ver definición en Apéndice C) tal que

$$
\phi_{I}^{-1}\left\{x_{I I}, \mathcal{U}(t), \phi_{I}\left[I_{p}\left(x_{I}{ }^{\prime}, x_{I I}{ }^{\prime}\right)^{\prime}, \mathcal{U}(t), b\right], b\right\}=x_{I}, \quad t \geq 0
$$

donde $I_{\mathrm{p}}$ es la permutación en columnas de la matriz identidad ( $\left.n \times n\right)$ que denota la partición $x_{\mathrm{I}}-x_{\mathrm{II}}$ del vector de estados $x$ :

$$
x=I_{p}\left(x_{I}{ }^{\prime}, x_{I I}\right)^{\prime}, \quad\left(x_{I}{ }^{\prime}, x_{I I}{ }^{\prime}\right)^{\prime}=\left(I_{l}, I_{I I}\right) x, \quad\left(I_{I}, I_{I I}\right)=I_{p}^{-1}
$$

De esta manera, la sustitución del conjunto de $\kappa$-ecuaciones algebraicas (3.7) en el sistema de $n$-ecuaciones de la planta (3.1) conduce a un sistema algebraicodiferencial:

$$
\begin{array}{r}
\dot{x}_{I I}^{*}=f_{I I}\left[x_{I I}^{*}, \mathcal{u}(t), y(t), b\right]:=f\left[I_{p}^{-1}\left\{\left(\phi_{I}^{-1}\right)^{\prime}\left[x_{I I}^{*}, \mathcal{U}(t), y(t), b\right],\left(x_{I I}^{*}\right)^{\prime}\right\}^{\prime}, u(t), b\right], \\
x_{I I o}^{*}=x_{I I o}=I_{I I} x_{o} \\
x^{*}(t)=I_{p}^{-1 \mid}\left\{\left(\phi_{I}^{-1}\right)^{\prime}\left[x_{I l}^{*}, \mathcal{U}(t), y(t), b\right],\left(x_{I I}^{*}\right)^{\prime}\right\}^{\prime} \in \Xi(t)=\left\{x \in \mathfrak{R}^{n} \mid y(t)=\phi_{I}[x, \mathcal{U}(t), b]\right\}
\end{array}
$$

que, dado el conjunto de datos $\left\{x_{\mathrm{II}}, y(t), u(t)\right\}$, producen una reconstrucción en linea exacta $x^{*}(t)$ de la trayectoria de la planta $x(t)$, siempre y cuando la trayectoria no observable

$$
x_{I I}^{*}(t)=\theta_{I I}\left[t, t_{o}, x_{I l o}, \mathcal{U}(t), y(t), b\right], \quad 0 \leq \operatorname{dim}\left(x_{I I}^{*}\right) \leq n-\kappa
$$

de la dinámica no observable $(n-\kappa)$-dimensional (3.16) sea E-estable. $\Xi(t)(3.17)$ está referida a la superficie no observable (variante en tiempo) puesto que, a menos que $\kappa$ $=n$, de la ecuación (3.7) no se puede decir en que lugar de $\Xi(t)$ se encuentra el estado de la planta $x$. La planta $x(t)$ y su trayectoria reconstruida $x^{*}(t)$ coinciden, pero sus propiedades de estabilidad no son necesariamente las mismas. Ya que $x^{*}(t)$ es la restricción de $x(t)$ en la superficie no observable $\Xi(t)$, y $x^{*}(t)$ tiene la propiedad de estabilidad de $x_{\mathrm{II}}^{*}(t)$ : si $x(t)$ es RE-estable, entonces $x_{\mathrm{II}}^{*}(t)$ es RE-estable; si $x(t)$ es Eestable, entonces $x_{\mathrm{II}}^{*}(t)$ es E-estable o RE-estable; si $x(t)$ es inestable, entonces $x_{\mathrm{II}}^{*}(t)$ es inestable, E-estable o RE-estable.

La trayectoria $x_{\mathrm{II}}^{*}(t)$ y la superficie $\Xi(t)$ no observables dependen de la estructura de estimabilidad $S$, definida por

$$
S=\left(k, x_{l}\right): \quad k=\left(\kappa_{1}, \ldots, \kappa_{m}\right)^{\prime}, \quad m \leq \kappa_{1}+\ldots+\kappa_{m}=\kappa \leq n, \quad x=I_{p}\left(x_{I}{ }^{\prime}, x_{I I}{ }^{\prime}\right)^{\prime}
$$


la cual esta formada por el vector de observabilidad $k$ y por la coordenada $x_{\text {II }}$ de la trayectoria no observable. En la siguiente definición se establece la noción de estimabilidad robusta, la cual es la base de la metodologia estructural del problema de estimación geométrico.

Observación 3.3. En general, el indice de observabilidad global $\kappa=\kappa_{1}+\ldots+\kappa_{m}$ determina el número de estados observables, siendo $(n-\kappa)$ el número de estados no observables; $x_{\mathrm{I}}$ es el conjunto de estados (observables) reconstruidos a partir de las salidas $y_{\mathrm{I}}$ y sus $\left(\kappa_{\mathrm{i}}-1\right)$ derivadas; y $x_{\mathrm{II}}$ es el conjunto de estados (no observables) reconstruidos a partir del modelo. De tal forma que, además de poder seleccionar el conjunto $k=\left(\kappa_{1}, \ldots, \kappa_{\mathrm{m}}\right)$ de manera combinatorial (Observación 3.2), la partición de estados $\left(x_{1}, x_{\mathrm{II}}\right)$ también puede ser seleccionada de diversas formas. De aqui que se haya definido la estructura de estimabilidad como $S=\left(k, x_{I}\right)$ (3.19). Por ejemplo, consideremos un reactor de homopolimerización con volumen constante, con: $x=(M$, $I, T)^{\prime}, \quad n=3 ; y=(M, T)^{\prime}, m=2$ (donde $\mathrm{M}$ : monómero, I: iniciador, T: temperatura). Entonces las estructuras $S=\left(k, x_{l}\right)$ obtenidas de forma combinatorial serian: $S_{1}=$ $\left\{(1,1),(M, I)^{\prime}\right\}, S_{2}=\left\{(1,1),(M, T)^{\prime}\right\}, S_{3}=\left\{(1,1),(I, T)^{\prime}\right\}, S_{4}=\left\{(2,1),(M, I, T)^{\prime}\right\}$, y $S_{5}=\{(1,2),(M, I$, $\left.T)^{\prime}\right\}$. Sin embargo, no todas estas estructuras son resolubles para la construcción del estimador (como se establecerá en la siguiente definición), y tampoco todas darán el mismo desempeño en el funcionamiento del estimador (como se verá en las secciones 3.6 y 3.7$)$.

Definición 2 (Estimabilidad robusta-exponencial). La trayectoria $x(t)$ (3.3)de la planta n-dimensional (3.1) es RE(robustamente exponencialmente)-estimable si existe una estructura de estimabilidad $S$ (3.19) tal que, a lo largo de la evolución de la planta $\mathrm{E}(t)(3.2)$, las siguientes propiedades se cumplen (uniformemente en $t$ ):

(i) El mapa $\phi_{\mathrm{I}}(x, \mathcal{U}, b)(3.11)$ es $\mathrm{Rx}_{\mathrm{I}}$-invertible y continuamente diferenciable en $(\mathcal{U}, b)$.

(ii) El mapa $\varphi(x, \mathcal{U}, v, b)(3.12)$ es continuamente diferenciable en $(x, \mathcal{U}, v, b)$.

(iii) La trayectoria no observable $x_{\mathrm{II}}^{*}(t)(3.18)$ es RE-estable.

Si $\kappa=n$ [es decir, no hay dinámica no observable, y por tanto la condición (iii) se cumple trivialmente), se dice que la trayectoria $x(t)$ es RE-observable. En caso contrario, si $\kappa<n$, se dice que la trayectoria $x(t)$ es RE-detectable. $\bullet$ 
La interpretación geométrica de las anteriores condiciones de estimabilidad y su comparación con los diseños existentes de observadores son principalmente que (Alvarez, 2000): la condición (i) es una propiedad de aplanabilidad- $\mathrm{RD}$ (robusto diferencial), relacionada a la involutibilidad robusta, de la superficie no observable $\Xi(t)$ (3.17) hacia la cual convergen los estados de un estimador geométrico; la condición (ii) asegura el acotamiento (equivalente a la condición de Lipschitz, ver Apéndice C) del término del error no lineal que interconecta las dinámicas del error (cuasi-lineal) observable y del error (no lineal) no observable del estimador geométrico; y la condición (iii) garantiza la estabilidad robusta de la trayectoria del estimador en la superficie no observable $\Xi(t)$. Por otra parte, la estructura de estimabilidad $S$ (3.19) determina las dimensiones de las superficies no observables $\Xi_{1}(t), \ldots, \Xi_{\mathrm{m}}(t)$, una para cada una de las salidas medidas (3.20), así como la representación de coordenadas $x_{\mathrm{II}}$ del estado no observable (3.15).

$$
\begin{gathered}
\Xi(t)=\bigcap_{i=1}^{m} \Xi_{i}(t) \\
\Xi_{i}(t)=\left\{x \in \mathfrak{R}^{n} \mid\left(y_{i}, \ldots, y_{i}^{\left(\kappa_{i}-1\right)}\right)^{\prime}(t)=\left(h_{i}, \ldots, L_{f}^{\kappa_{i}-1} h_{i}\right)^{\prime}[x, \mathcal{U}(t), b]\right\}, \quad \operatorname{dim}\left[\Xi_{i}(t)\right]=n-\kappa_{i}
\end{gathered}
$$

Observación 3.4. En la Definición 2, la condición (i) significa que a partir de las salidas $(y)$ y de sus derivadas (esto es, el mapa $\phi_{1}$ ) es posible reconstruir el mapa $x_{\mathrm{I}}$. La condición (ii) significa que las $\kappa_{i}$-ésimas derivadas de las salidas $y_{i}$ estén acotadas, lo cual generalmente se cumple en los procesos químicos (ya que por las condiciones fisicas, todas las variables y sus funciones son finitas). La condición (iii) significa que la dinámica $\left(\dot{x}_{I I}^{*}\right)$ de los estados no observables seleccionados sea estable. De acuerdo a estas condiciones y utilizando el ejemplo definido en la Observación 3.3: Las estructuras $S_{1}=\left\{(1,1),(M, I)^{\prime}\right\}$ y $S_{3}=\{(1,1),(I, T)\}$ no cumplen con la condición (i) ya que tienen $\operatorname{det}\left(\phi_{I_{x_{l}}}\right)=0$, y por lo tanto no son $\mathrm{x}$-invertible y se descartan como posibles estructuras candidatas. El resto de las estructuras si cumplen con las tres condiciones, correspondiendo la estructura $S_{2}=\left\{(1,1),(M, T)^{\prime}\right\}$ a un caso detectable, con $x_{\mathrm{II}}=(\eta)$ (que como se sabe la dinámica del iniciador es lineal, y por lo tanto es RE-estable); mientras que las estructuras $S_{4}=\left\{(2,1),(M, I, T)^{\prime}\right\}$, y $S_{5}=\left\{(1,2),(M, I, T)^{\prime}\right\}$ corresponden a casos observables (cumpliendo trivialmente la condición (iii)). En las siguientes secciones se darán hipótesis para definir cual de estas tres estructuras que cumplieron con la Definición 2 será la más adecuada para obtener el mejor desempeño del estimador. 


\subsection{Estimador-P (propor cional) no lineal}

En esta sección, se construye el estimador-P y luego se establece la dinámica del error de estimación, el criterio de convergencia robusta, y la técnica de sintonización. Para la construcción se ha tomado como referencia a trabajos publicados recientemente (Alvarez, 1996, 2000), donde se ha discutido la construcción y su interpretación geométrica, asi como diversos aspectos sobre el criterio de convergencia.

225999

\subsubsection{Construcción}

Consideremos a las entradas exógenas aumentadas $\mathcal{U}(3.10)$ como el estado $x_{\mathrm{u}}$

$$
\begin{aligned}
& \mathcal{u}=\left[u_{1}, \dot{u}_{1}, \ldots, u_{1}^{\left(v_{1}-1\right)} ; \ldots ; u_{p}, \dot{u}_{p}, \ldots, u_{p}^{\left(v_{p}-1\right)}\right]^{\prime}:=x_{u} \\
& x_{u}=\left[x_{u 1}, x_{u 2}, \ldots, x_{u\left(v_{1}\right)} ; \ldots ; x_{u\left(v_{1}+\cdots+v_{p-1}+1\right)}, x_{u\left(v_{1}+\cdots+v_{p-1}+2\right)}, \ldots, x_{u\left(v_{1}+\cdots+v_{p}\right)}\right]^{\prime}
\end{aligned}
$$

del exo-sistema con dinámica lineal (las matrices $\Gamma_{\mathrm{u}}, \Pi_{\mathrm{u}}, \mathrm{y} \Delta_{\mathrm{u}}$ están definidas en el Apéndice D)

$$
\begin{gathered}
\dot{x}_{u}=\left[x_{u 2}, x_{u 3}, \ldots, u_{1}^{\left(v_{1}\right)} ; \ldots ; x_{u\left(v_{1}+\cdots+v_{p-1}+2\right)}, x_{u\left(v_{1}+\cdots+v_{p-1}+3\right)}, \ldots, u_{p}^{\left(v_{p}\right)}\right]^{\prime} \\
\dot{x}_{u}=\Gamma_{u} x_{u}+\Pi_{u} v(t), \quad u=\Delta_{u} x_{u}, \quad v(t)=\left[u_{1}^{\left(v_{1}\right)}, \ldots, u_{p}^{\left(v_{p}\right)}\right]^{\prime},\|v(t)\| \leq \varepsilon_{u}
\end{gathered}
$$

Por lo que ahora la planta (3.1) aumentada con este nuevo estado es:

$$
\begin{array}{ll}
\dot{x}_{u}=\Gamma_{u} x_{u}+\Pi_{u} \mathrm{v}(t), & u=\Delta_{u} x_{u} \\
\dot{x}=f\left(x, \Delta_{u} x_{u}, b\right), & y=h(x, b), \quad z=h_{z}(x, b)
\end{array}
$$

Ya que el mapa (3.11) define el vector $x_{\mathrm{I}}$ (3.14), y además existe un mapa $x_{\mathrm{II}}(3.15)$ no observable que definiremos como

$$
x_{I I}:=\phi_{I I}(x)
$$

entonces el mapa de estimabilidad

$$
\phi\left(x, x_{u}, b\right)=\left[\phi_{I}{ }^{\prime}\left(x, x_{u}, b\right), \phi_{I I}{ }^{\prime}(x)\right]^{\prime}
$$

de acuerdo a la Definición 2, es Rx-invertible

$$
\phi^{-1}\left[\phi\left(x, x_{u}, b\right), x_{u}, b\right]=x(t)
$$

y continuamente diferenciable o L (Lipschitz)-continuo (ver definición en Apéndice C), y por tanto $\phi$ y $\phi^{-1}$ están acotados:

$$
\left\|\phi\left(\hat{x}, \hat{x}_{u}, \beta\right)-\phi\left(x, x_{u}, b\right)\right\| \leq D_{x}\|\hat{x}-x\|+D_{u}\left\|\hat{x}_{u}-x_{u}\right\|+D_{b}\|\beta-b\|
$$




$$
\begin{aligned}
& \left\|\phi^{-1}\left[\phi\left(\hat{x}, \hat{x}_{u}, \beta\right), \hat{x}_{u}, \beta\right]-\phi^{-1}\left[\phi\left(x, x_{u}, b\right), x_{u}, b\right]\right\| \leq \\
& F_{z}\left\|\phi\left(\hat{x}, \hat{x}_{u}, \beta\right)-\phi\left(x, x_{u}, b\right)\right\|+F_{u}\left\|\hat{x}_{u}-x_{u}\right\|+F_{b}\|\beta-b\|
\end{aligned}
$$

Con base en la propiedad de estimabilidad (Definición 2), específicamente en la condición (i) relacionada a una propiedad de aplanabilidad, se empleará un cambio de coordenadas para llevar a la planta aumentada (3.23) a un sistema casi-lineal. El cambio de coordenadas $z$ está descrito de forma natural por el estado del exo-sistema $x_{u}(3.22)$ y el mapa de estimabilidad $\phi(3.25)$ :

$$
z=\left[\begin{array}{c}
z_{u} \\
z_{l} \\
z_{I I}
\end{array}\right]:=\left[\begin{array}{c}
x_{u} \\
\phi_{I}\left(x, x_{u}, b\right) \\
\phi_{I I}(x)
\end{array}\right]
$$

Con el cual la planta aumentada (3.23) en coordenadas- $z$ adquiere la forma (las matrices $\Gamma_{\mathrm{o}}, \Pi_{\mathrm{o}}, \mathrm{y} \Delta_{\mathrm{o}}$, así como los mapas $\varphi$ y $\theta$ están definidos en el Apéndice D):

$$
\begin{array}{|lll}
\hline \dot{z}_{u}=\Gamma_{u} z_{u}+\Pi_{u} \cup(t), & u=\Delta_{u} x_{u} ; & \operatorname{dim}\left(z_{u}\right)=v \\
\dot{z}_{I}=\Gamma_{o} z_{I}+\Pi_{o} \varphi\left(z_{I}, z_{I I}, z_{u}, \cup, b\right), & y=\Delta z_{I} ; & \operatorname{dim}\left(z_{I}\right)=\kappa \\
\dot{z}_{I I}=\theta\left(z_{I}, z_{I I}, z_{u}, b\right) ; & & \operatorname{dim}\left(z_{I I}\right)=n-\kappa
\end{array}
$$

donde $z_{\mathrm{u}}, z_{\mathrm{I}}$, y $z_{\mathrm{II}}$ son los estados exo-observables, observables, y no observables de la planta, respectivamente; $y$ además los pares de matrices $\left(\Gamma_{u}, \Delta_{u}\right)$ y $\left(\Gamma_{o}, \Delta_{0}\right)$ son observables.

La dinámica no observable (3.16)-(3.17), en coordenadas originales, forzada con $\left(z_{\mathrm{u}}, z_{\mathrm{l}}\right)=[\mathcal{U}(t), y(t)]$ corresponde a la dinámica no observable en coordenadas- $z$

$$
\dot{z}_{l l}^{*}=\theta\left\{\left[\mathcal{U}(t), y(t), z_{I I}^{*}\right], \cup(t), b\right\}, \quad z^{*}(t)=\left[y(t), z_{I I}^{*}\right]^{\prime} \in \Sigma(t)=\left\{z \in \mathbb{Z} \mid z_{l}=y(t)\right\}
$$

cuya solución $z_{I l}^{*}(t)$ es la trayectoria no observable RE-estable (3.18) en el hiperplano $\Sigma(t)$ [que corresponde a la superficie $\Xi(t),(3.17)$, en coordenadas-z], tal que

$$
\begin{aligned}
& \left\|\hat{z}_{I I}^{*}(t)-z_{I I}^{*}(t)\right\| \leq A_{I I}\left\|\hat{z}_{I I o}^{*}-z_{I I o}^{*}\right\| e^{-L_{I I}\left(t-t_{o}\right)} \\
& A_{I I}=A_{x} C_{x}^{\phi}, \quad a_{I I}=\lim _{\hat{x}(t) \rightarrow x(t)} A_{I I}
\end{aligned}
$$

donde $\mathrm{C}_{\mathrm{x}}^{\phi}$ es el número de condición más grande, a lo largo de la trayectoria, de la matriz jacobiana $\phi_{\mathrm{x}}=\partial \phi / \partial \mathrm{x}\left[D_{\mathrm{x}} \mathrm{y} F_{\mathrm{z}}\right.$ definidas en (3.27) y (3.28)]: 


$$
C_{x}^{\phi}(N)=\sup _{\left(\hat{x}, \hat{x}_{u}, \beta\right) \in N}\left(\frac{D_{x}}{F_{z}}\right), \quad c_{x}^{\phi}=\lim _{e \rightarrow 0} C_{x}^{\phi}
$$

$A_{\mathrm{x}}$ y $L_{\mathrm{Il}}$ son los parámetros de amplitud y de decaimiento de la siguiente desigualdad correspondiente a la convergencia- $\mathrm{E}$ de la trayectoria no observable perturbada en la condición inicial:

$$
\begin{aligned}
& \left\|\hat{x}^{*}(t)-x^{*}(t)\right\| \leq A_{x}\left\|\hat{x}_{o}-x_{o}\right\| e^{-L_{I I}\left(t-t_{o}\right)} \\
& \left(a_{x}, \lambda_{I I}\right)=\lim _{\hat{x}(t) \rightarrow x(t)}\left(A_{x}, L_{I I}\right)
\end{aligned}
$$

La observabilidad de las matrices $\left(\Gamma_{\mathrm{u}}, \Delta_{\mathrm{u}}\right)$ y $\left(\Gamma_{\mathrm{o}}, \Delta_{\mathrm{o}}\right)$ de la planta aumentada (3.30), y la RE-estabilidad de la trayectoria no observable (3.31) sugieren el siguiente sistema como estimador-P de la planta:

$$
\begin{array}{ll}
\dot{\hat{z}}_{u}=\Gamma_{u} \hat{z}_{u}+K_{u}\left(u-\Delta_{u} \hat{z}_{u}\right), & \Psi_{u}=\Delta_{u} \hat{z}_{u} \\
\dot{\hat{z}}_{I}=\Gamma_{o} \hat{z}_{I}+\Pi_{o} \varphi\left[\hat{z}_{I}, \hat{z}_{I I}, \hat{z}_{u}, \cup(t), \beta\right]+K_{o}\left(y-\Delta_{o} \hat{z}_{I}\right), & \psi=\Delta_{o} \hat{z}_{I} \\
\dot{\hat{z}}_{I I}=\theta\left[\hat{z}_{I}, \hat{z}_{I I}, \hat{z}_{u}, \cup(t), \beta\right] & \\
\hline
\end{array}
$$

donde $\left(\psi, \psi_{\mathrm{u}}\right)$ son los estimados de las mediciones $(y, u)$.

Aplicando nuevamente el cambio de coordenadas, $\left(\hat{x}_{u}{ }^{\prime}, \hat{x}^{\prime}\right)^{\prime}=\phi^{-1}(\hat{z}, \beta)$, se obtiene el siguiente estimador no lineal en coordenadas originales:

$$
\begin{aligned}
& \dot{\hat{x}}_{u}=A_{u} \hat{x}_{u}+K_{u} u, \quad \hat{u}=\Delta_{u} \hat{x}_{u} \\
& \dot{\hat{x}}=f\left(\hat{x}, \Delta_{u} \hat{x}_{u}, \beta\right)+G\left(\hat{x}, \hat{x}_{u}, \beta\right)[y-h(\hat{x}, \beta)], \quad \hat{y}=h(\hat{x}, \beta), \quad \hat{z}=h_{z}(\hat{x}, \beta)
\end{aligned}
$$

donde $A_{u}=\Gamma_{u}-K_{u} \Delta_{u}$ es una matriz estable, y $G\left(\hat{x}, \hat{x}_{u}, \beta\right)$ es una matriz de ganancias dada por:

$$
G\left(\hat{x}, \hat{x}_{u}, \beta\right)=\left[I_{11}{ }^{\prime}, I_{21}{ }^{\prime}\right]^{\prime} \phi_{I x_{i}}^{-1}\left(\hat{x}, \hat{x}_{u}, \beta\right) K_{o}\left(s_{o}\right)
$$

donde el par las matrices I11, I21 están dadas por [ver ecuación (3.15)]:

$$
I_{p[n \times n]}^{-1}=\left[\begin{array}{c}
I_{I[\mathrm{\kappa} \times n]} \\
I_{I[(n-\mathrm{k}) \times n]}
\end{array}\right]:=\left[\begin{array}{cc}
I_{11[\mathrm{\kappa} \times \mathrm{\kappa}]} & I_{12[\mathrm{\kappa} \times(n-\mathrm{\kappa})} \\
I_{21[(n-\mathrm{\kappa}) \times \mathrm{\kappa}]} & I_{22[(n-\mathrm{\kappa}) \times(n-\mathrm{\kappa})}
\end{array}\right]
$$


Observación 3.5. El estimador-P (3.35) consiste de: (a) una réplica de la planta (término predictor) evaluada con los valores aproximados de los estados $(\hat{z})$ y parámetros $(\beta)$, y (b) la adición de un término (corrector) forzado por la diferencia entre la medición y su estimado (innovación) para las dinámicas exo-observables y observables de la planta. En general, esta forma es común para cualquier estimador (detector u observador), siendo la principal diferencia la técnica de selección de la ganancia G. Por ejemplo, el FEK (filtro extendido de Kalman) emplea la estadística (minimización del error de covariancia), mientras que el estimador propuesto emplea la teoria del control geométrico no lineal.

La selección de la matriz $G$ está determinada por las siguientes consideraciones (Alvarez, 1999; Alvarez y López, 1999]:

(i) el estimador-P (3.36) debe generar una trayectoria $\hat{x}(t)$ E-convergente,

(ii) la dinámica del error de estimación observable debe ser cuasi-lineal, y

(iii) la dinámica del error de estimación no observable debe introducir la inyección de las salidas medidas.

La verificación de estas tres condiciones se establecerá en las siguientes dos subsecciones.

\subsubsection{Dinámica del error de estimación}

Definiendo el error de estimación en coordenadas- $z$ como, $e=\left[e_{1}, e_{I I}, e_{u}, e_{b}\right]$ :

$$
\begin{array}{lll}
e_{u}=\hat{z}_{u}-z_{u} & & \text { (error del exo-sistema) } \\
e_{I}=\hat{z}_{I}-z_{I} & & \text { (error observable) } \\
e_{I I}=\hat{z}_{I I}-z_{I I} & & \text { (error no observable) } \\
e_{b}=\beta-b, \quad\left\|e_{b}\right\| \leq \varepsilon_{b} & & \text { (error de los parametros) }
\end{array}
$$

y restando (3.30) de (3.35) se obtiene la siguiente dinámica del error

$$
\begin{array}{|ll}
\dot{e}_{u}=A_{u} e_{u}-\Pi_{u} v(t), & A_{u}=\Gamma_{u}-K_{u} \Delta_{u}, \quad \mu=\Delta_{u} e_{u} \\
\dot{e}_{I}=A_{o} e_{I}+\Pi_{o} q_{I}\left(e_{I}, e_{I I}, e_{u}, e_{b}, t\right), & A_{o}=\Gamma_{o}-K_{o} \Delta_{o}, \quad v=\Delta_{o} e_{I} \\
\dot{e}_{I I}=\omega\left(e_{I I}, t\right)+q_{I I}\left(e_{I}, e_{I I}, e_{u}, e_{b}, t\right) &
\end{array}
$$

donde $v(t)$ es una perturbación L-continua, y acotada de acuerdo a:

$$
\|v(t)\| \leq \varepsilon_{v}
$$


$q_{I}\left(e_{I}, e_{I I}, e_{u}, e_{b}, t\right)$ y $q_{I I}\left(e_{I}, e_{I I}, e_{u}, e_{b}, t\right)$ (definidos en el Apéndice D) son perturbaciones desvanecientes en $\left(e_{I}, e_{I I}, e_{u}, e_{b}\right)=(0,0,0,0)$, esto es

$$
q_{I}(0,0,0,0, t)=q_{I I}\left(0, e_{I I}, 0,0, t\right)=0
$$

Estas perturbaciones, además de ser desvanecientes, son L-continuas (acotadas), esto es:

$$
\begin{gathered}
\left\|q_{I}\left(e_{I}, e_{I I}, e_{u}, e_{b}, t\right)\right\| \leq M_{I}\left\|e_{I}(t)\right\|+M_{I I}\left\|e_{I I}(t)\right\|+M_{u}\left\|e_{u}(t)\right\|+M_{b}\left\|e_{b}\right\| \\
\left\|q_{I I}\left(e_{I}, e_{I I}, e_{u}, e_{b}, t\right)\right\| \leq N_{I}\left\|e_{I}(t)\right\|+N_{u}\left\|e_{u}(t)\right\|+N_{b}\left\|e_{b}\right\| \\
\left(m_{I}, m_{I I}, n_{I}\right)=\lim _{e_{I}, e_{I I}, e_{u}, e_{b} \rightarrow 0}\left(M_{I}, M_{I I}, N_{I}\right)
\end{gathered}
$$

Asimismo, la observabilidad del par de matrices $\left(\Gamma_{\mathrm{u}}, \Delta_{\mathrm{u}}\right)$ y $\left(\Gamma_{\mathrm{o}}, \Delta_{\mathrm{o}}\right)$ de la planta aumentada (3.30), garantizan la existencia de matrices de ganancias $\left(\mathrm{K}_{\mathrm{u}}, \mathrm{K}_{\mathrm{o}}\right)$ tal que los matrices $\left(A_{\mathrm{u}}, A_{\mathrm{o}}\right)$ sean estables, respectivamente.

Sean las matrices de ganancias ajustables del estimador de la siguiente forma parametrizada:

$$
\begin{aligned}
& K_{u}\left(s_{u}\right)=d b\left[\left(s_{u} k_{11}^{u}, \ldots, s_{u}^{v_{1}} k_{v_{1}}^{u}\right)^{\prime}, \ldots,\left(s_{u} k_{1 p}^{u}, \ldots, s_{u}^{v_{p}} k_{v_{p} p}^{u}\right)^{\prime}\right], \quad s_{u}>0 \\
& K_{o}\left(s_{o}\right)=d b\left[\left(s_{o} k_{11}^{o}, \ldots, s_{o}^{\kappa_{1}} k_{\kappa_{1} 1}^{u}\right)^{\prime}, \ldots,\left(s_{o} k_{1 m}^{o}, \ldots, s_{o}^{\kappa_{m}} k_{\kappa_{m} m}^{o}\right)^{\prime}\right], \quad s_{o}>0
\end{aligned}
$$

con entradas de referencia, $\mathrm{K}_{\mathrm{u}}(1)$ y $\mathrm{K}_{\mathrm{o}}(1)$, tales que hacen estable la dinámica del error de salida $\left[\mu=\Delta_{u} e_{u}:=\hat{u}-u\right.$, y $\nu=\Delta_{o} e_{I}:=\hat{y}-y$ del sistema dinámico (3.40)]:

$$
\begin{array}{lll}
\mu_{i}^{\left(v_{i}\right)}+k_{1 i}^{u} \mu_{i}^{\left(v_{i}-1\right)}+\ldots+k_{v_{i} i}^{u} \mu_{i}=0, & 1 \leq i \leq p ; & \mu_{i}=\hat{u}_{i}-u_{i} \\
v_{i}^{\left(\kappa_{i}\right)}+k_{1 i}^{o} v_{i}^{\left(\kappa_{i}-1\right)}+\ldots+k_{\kappa_{i} i}^{o} v_{i}=0, & 1 \leq i \leq m ; & v_{i}=\hat{y}_{i}-y_{i}
\end{array}
$$

las cuales son LNPA (lineal, no interactiva, y de polos asignables), tal que las matrices $\left(A_{\mathrm{u}}\left(s_{\mathrm{u}}\right), A_{\mathrm{o}}\left(s_{\mathrm{o}}\right)\right)$ cumplen las siguientes desigualdades:

$$
\begin{aligned}
& \left\|e^{A_{u}\left(s_{u}\right) t}\right\| \leq a_{u} e^{-s_{u} \lambda_{u} t}, \quad t \geq 0 \\
& \left\|e^{A_{o}\left(s_{o}\right) t}\right\| \leq a_{o} e^{-s_{o} \lambda_{o} t}, \quad t \geq 0
\end{aligned}
$$

donde $\left(a_{u}, \lambda_{u}\right)$ y $\left(a_{o}, \lambda_{o}\right)$ están determinadas por $\mathrm{K}_{\mathrm{u}}(1)$ y $\mathrm{K}_{\mathrm{o}}(1)$, y son independientes de los parámetros de ajuste (de aceleración o retardo) $s_{\mathrm{u}}$ y $s_{\mathrm{o}}$. 


\subsubsection{Convergencia}

La dinámica del error de estimación (3.40) con perturbaciones $\left(u, q_{I}, q_{I I}\right)=(0,0,0)$ es RE-estable. Por lo que la convergencia-RE del estimador consiste en encontrar condiciones para que la dinámica del error pueda tolerar dichas perturbaciones. En el siguiente teorema se resume la construcción de los estimador-P, y se dan condiciones suficientes para su convergencia en función de los parámetros de ajuste $s_{\mathrm{u}} \mathrm{y} s_{\mathrm{o}}$.

Teorema 1 (Prueba en Apéndice E.). Sea la trayectoria $x(t)$ de la planta (3.1) REestimable, de acuerdo a la Definición 2, y sean las ganancias de referencia (3.46), $\mathrm{K}_{\mathrm{u}}$ (1) y $\mathrm{K}_{\mathrm{o}}(1)$, que hacen estable la dinámica LNPA del error de salida (3.47). Entonces, el estimador-P

$$
\begin{aligned}
& \dot{\hat{x}}_{u}=\Gamma_{u} \hat{x}_{u}+K_{u}\left(u-\Delta_{u} \hat{x}_{u}\right), \quad \hat{u}=\Delta_{u} \hat{x}_{u} \\
& \dot{\hat{x}}=f\left(\hat{x}, \Delta_{u} \hat{x}_{u}, \beta\right)+G\left(\hat{x}, \hat{x}_{u}, \beta\right)[y-h(\hat{x}, \beta)], \quad \hat{y}=h(\hat{x}, \beta), \quad \hat{z}=h_{z}(\hat{x}, \beta)
\end{aligned}
$$

con ganancias parametrizadas $\mathrm{K}_{\mathrm{u}}\left(s_{\mathrm{u}}\right)$ y $\mathrm{K}_{\mathrm{o}}\left(s_{\mathrm{o}}\right)(3.46)$, y la ganancia no lineal

$$
G\left(\hat{x}, \hat{x}_{u}, \beta\right)=\left[I_{11}{ }^{\prime}, I_{21}{ }^{\prime}\right]^{\top} \phi_{I x_{I}}^{-1}\left(\hat{x}, \hat{x}_{u}, \beta\right) K_{o}\left(s_{o}\right)
$$

genera una trayectoria $\hat{x}(t)$ que RE-converge a $x(t)$, si el parámetro $s_{\mathrm{o}}$ es suficientemente grande tal que

$$
\begin{array}{lr}
s_{o} \lambda_{o}>a_{o} m_{l} & \text { si } \kappa=n, \quad \text { o } \\
s_{o} \lambda_{o}>a_{o} m_{l}+a_{o} c_{x}^{\phi}\left(a_{x} / \lambda_{I I}\right) m_{I I} n_{I} & \text { si } \kappa<n
\end{array}
$$

donde $\mathrm{c}_{\mathrm{x}}^{\phi},\left(a_{\mathrm{x}}, \lambda_{\mathrm{II}}\right),\left(m_{\mathrm{I}}, m_{\mathrm{II}}, n_{\mathrm{I}}\right), \mathrm{y}\left(a_{\mathrm{o}}, \lambda_{\mathrm{o}}\right)$ están definidas en las ecuaciones (3.33), (3.34), (3.43)-(3.45), y (3.49), respectivamente.

Observación 3.6. En este teorema el par $\left(a_{x}, \lambda_{\text {II }}\right)$ está fijado por la trayectoria no observable, el par $\left(a_{0}, \lambda_{0}\right)$ están determinados por la ganancia $K_{\mathrm{o}}$ del estimador (definidas a su vez por la dinámica del error de salida), las constantes $\left(m_{\mathrm{I}}, m_{\mathrm{Il}}, n_{\mathrm{I}}\right)$ están determinadas y acotadas por la dinámica del error de estimación, y el número de condición $\mathrm{c}_{\mathrm{x}}^{\phi}$ está determinado por la estructura de estimabilidad $S$ seleccionada y por la trayectoria de operación. El significado de los términos involucrados en la condición de convergencia es el siguiente, para el caso observable y estimable, respectivamente:

$$
\underbrace{s_{o} \lambda_{o}}_{\begin{array}{c}
\text { margen de } \\
\text { estabilización }
\end{array}}>\underbrace{a_{o} m_{l}}_{\begin{array}{c}
\text { margen de } \\
\text { auto-desestabilización } \\
\text { de la dinámica observable }
\end{array}}
$$




$$
\underbrace{s_{o} \lambda_{o}}_{\begin{array}{c}
\text { margen de } \\
\text { estabilización }
\end{array}}>\underbrace{a_{o} m_{I}}_{\begin{array}{c}
\text { margen de } \\
\text { auto-desestabilización } \\
\text { de la dinámica observable }
\end{array}}+\underbrace{a_{o} c_{x}^{\phi}\left(a_{x} / \lambda_{I I}\right) m_{I I} n_{l}}_{\begin{array}{c}
\text { potencial de desestabilización debido } \\
\text { al acoplamiento entre las dinámicas } \\
\text { de error observable y no observable }
\end{array}}
$$

Como puede observarse el parámetro libre a ajustar para garantizar que estas condiciones se cumplan es el parámetro $s_{o}$, el cual como se indica en el Teorema 1 debe ser suficientemente grande para garantizar convergencia. En la práctica se utiliza como referencia la dinámica natural de la planta, y el parámetro de ajuste se selecciona como $s_{o} \approx 10$, lo cual significa que el estimador es 10 veces más rápido de la dinámica natural del proceso.

En el siguiente corolario del Teorema 1, se presentan las desigualdades explícitas que caracterizan la convergencia-RE del estimador-P.

Corolario del Teorema 1. Sea la trayectoria $x(t)$ de la planta (3.1) RE-estimable, de acuerdo a la Definición 2, y sea la condición (desigualdad) (3.52) del Teorema 1 que se cumple con $s_{\mathrm{o}}$ y $s_{\mathrm{u}}$ tal que

$$
\begin{array}{ll}
l_{u}\left(s_{u}\right)>L_{I}\left(s_{o}\right) & \text { si } \kappa<n, \text { o } \\
l_{u}\left(s_{u}\right)>L_{I}\left(s_{o}\right)>L_{I I} & \text { si } \kappa=n
\end{array}
$$

Entonces, los estados, las entradas y las salidas estimados convergen-RE como sigue:

$$
\begin{aligned}
& \left\|\hat{x}_{u}(t)-x_{u}(t)\right\| \leq \quad \sigma_{u o} e^{-l_{u}\left(t-t_{o}\right)}+b_{u} \\
& \|\hat{x}(t)-x(t)\| \leq\left(c_{\sigma}^{x} \sigma_{a o}+c_{u}^{x} \sigma_{u o}\right) e^{-L_{x}\left(t-t_{o}\right)}+b_{x} \\
& \|\hat{y}(t)-y(t)\| \leq\left(c_{\sigma}^{y} \sigma_{a o}+c_{u}^{y} \sigma_{u o}\right) e^{-L_{l}\left(t-t_{o}\right)}+b_{y}
\end{aligned}
$$

con factores de decaimiento

$$
l_{u}=s_{u} \lambda_{u}, \quad L_{I}=s_{o} \lambda_{o}-a_{o} M_{l} \quad\left\{\begin{array}{l}
L_{x}=L_{l} \text { si } \kappa<n \\
L_{x}>L_{l I} \text { si } \kappa=n
\end{array}\right.
$$

$\mathrm{y}$ cotas últimas

$$
b_{u}=g_{u} \varepsilon_{v}, \quad b_{x}=b_{v}^{x} \varepsilon_{v}+b_{b}^{x} \varepsilon_{b}, \quad b_{y}=b_{v}^{y} \varepsilon_{v}+b_{b}^{y} \varepsilon_{b}
$$

$\left(\sigma_{u o}, \sigma_{a o}, c_{\sigma}^{x}, c_{u}^{x}, c_{\sigma}^{y}, c_{u}^{y}, g_{u}, b_{u}^{x}, b_{b}^{x}, b_{u}^{y}, \mathrm{y} b_{b}^{y}\right.$ están definidos en Apéndice E). $\bullet$

De acuerdo a este corolario, escogiendo $s_{\mathrm{o}}$ y $s_{\mathrm{u}}$ suficientemente grandes: (a) los factores de decaimiento $l_{\mathrm{u}}$ y $L_{\mathrm{l}}$ (3.55) se pueden hacer arbitrariamente grandes 
(rápidos), (b) las cotas últimas $b_{\mathrm{u}}$ y $b_{\mathrm{y}}$ (3.56) pueden hacerse arbitrariamente pequeñas, (c) el factor de decaimiento $L_{\mathrm{x}}$ (3.55) y la cota última $b_{\mathrm{x}}$ (3.56) pueden hacerse arbitrariamente cerca de sus valores limites $L_{x}^{*}$ y $b_{x}^{*}$, respectivamente, y dados por:

$$
\begin{array}{ll}
\left(b_{x}^{*}, L_{x}^{*}\right)=\left(F_{b} \varepsilon_{b}, L_{I}\right) & \text { si } \kappa=n, 0 \\
\left(b_{x}^{*}, L_{x}^{*}\right)=\left(\frac{A_{a}}{L_{I I}} F_{z}\left\|B_{b}\right\| \varepsilon_{b}+F_{b}, L_{I}\right) & \text { si } \kappa<n
\end{array}
$$

y (d) la respuesta exponencial del error de estados $[\|\hat{x}(t)-x(t)\|$ en (3.54)] es proporcional al número de condición $c_{\phi}^{x}$ (3.33) del mapa de estimabilidad $\phi$ (3.25) a lo largo de la trayectoria, de acuerdo a la siguiente expresión:

$$
c_{\sigma}^{x} \sigma_{a o}=a_{\sigma} A_{a}\left[c_{x}^{\phi}\left\|\hat{x}_{o}-x_{o}\right\|+F_{z} D_{u}\left\|\hat{x}_{u o}-x_{u o}\right\|+F_{z} D_{r} \varepsilon_{r}\right]
$$

Observación 3.7. Este corolario muestra que efectivamente los estados estimados y las salidas estimadas $\left(\hat{x}_{u}, \hat{x}, \hat{y}\right)$ convergen exponencialmente a sus valores reales $\left(x_{\mathrm{w}}\right.$ $x, y)$ con cotas últimas muy pequeñas $\left(b_{u^{\prime}}, b_{x^{\prime}}, b_{y}\right)$. Siendo estas cotas función de los errores en las condiciones iniciales de los estados-entradas-parámetros, así como de la adecuada la sintonización del exo-observador - estimador (i.e. selección de $K_{\mathrm{u}}\left(s_{\mathrm{u}}\right)$ y $K_{\mathrm{o}}\left(s_{\mathrm{o}}\right)$ ).

\subsection{Estimador-PI (proporcional integral) no lineal}

En esta sección se presenta la versión PI (proporcional integral) del estimador geométrico no lineal. La acción integral se incluye con la finalidad (semejante al control lineal) de eliminar los sesgos en las salidas estimadas $(\hat{y})$, reducir los sesgos en los estados estimados $(\hat{x}$ y $\hat{z}$ ), compensar tanto las perturbaciones persistentes causadas por errores en los parámetros del modelo $(b-\beta)$, y atenuar las perturbaciones causadas por ruido en las salidas medidas.

\subsubsection{Construcción}

La justificación de la adición integral es la siguiente: Ya que las trayectorias de los errores exo-observables y observables $\left[e_{\mathrm{u}}\right.$ y $e_{\mathrm{l}}$ en (3.40)] son más rápidas que la 
trayectoria no observable $\left[e_{\mathrm{II}}\right.$ en $\left.(3.40)\right]$, entonces la perturbación $q_{I}$ de la dinámica del error observable [en (3.40)] puede ser reescrita como sigue $\left(q_{r}\right.$ y $q_{l}$ son definidos en Apéndice D):

$$
q_{I}\left(e_{I}, e_{I I}, e_{u}, \mathrm{v}, e_{b}, t\right)=q_{r}\left(e_{I}, e_{u}, \mathrm{v}, t\right)+q_{l}\left(e_{I I}, e_{b}, t\right)
$$

donde $q_{r}$ es la parte rápida de la perturbación en la dinámica observable, debido a errores en la partición de estados observables y salidas exógenas; y $q_{l}$ la parte lenta de la perturbación en la dinámica observable, debido a errores en la partición no observable y en los parámetros del modelo. Todas las perturbaciones son desvanecientes en $\left(e_{I}, e_{I I}, e_{u}, v, e_{b}\right)=(0,0,0,0,0)$. Esto es

$$
q_{r}(0,0,0, t)=q_{l}(0,0, t)=0
$$

De acuerdo a la partición anterior, $q_{l}$ es una perturbación lenta persistente que puede considerarse como un estado adicional:

con dinámica

$$
z_{q}=q_{l}\left(e_{I I}, e_{b}, t\right)
$$

$$
\dot{z}_{q}=0
$$

para el cual se puede obtener un estimador rápido, $\hat{z}_{q}$, a partir de una serie de integradores (uno para cada salida $y$ ):

$$
\dot{\hat{z}}_{q}=K_{I}\left(s_{o}\right)[y-h(\hat{x}, \beta)], \quad \hat{z}_{q}\left(t_{o}\right)=0 ; \quad K_{l}\left(s_{o}\right)=\operatorname{diag}\left(s_{o}^{\kappa_{1}+1} k_{1}^{l}, \ldots, s_{o}^{\kappa_{m}+1} k_{m}^{l}\right)
$$

Con la definición del nuevo estado $z_{q}$ y su estimado $\hat{z}_{q}$, se obtiene que el estimadorPI en coordenadas- $z$ está dado por

$$
\begin{array}{|lr}
\dot{\hat{z}}_{u}=\Gamma_{u} \hat{z}_{u}+K_{u}\left(\Delta_{u} z_{u}-\Delta_{u} \hat{z}_{u}\right), & \Psi_{u}=\Delta_{u} \hat{z}_{u} \\
\dot{\hat{z}}_{q}=K_{I}\left(s_{o}\right)\left(\Delta_{o} z_{I}-\Delta_{o} \hat{z}_{I}\right) & \\
\dot{\hat{z}}_{I}=\Gamma_{o} \hat{z}_{I}+\Pi_{o} \varphi\left(\hat{z}_{I}, \hat{z}_{I I}, \hat{x}_{u}, \cup, \beta\right)+K_{o}\left(\Delta_{o} z_{I}-\Delta_{o} \hat{z}_{I}\right)+\hat{z}_{q}, \quad \psi=\Delta_{o} \hat{z}_{I} \\
\dot{\hat{z}}_{I I}=\theta\left(\hat{z}_{I}, \hat{z}_{I I}, \hat{x}_{u}, \beta\right) &
\end{array}
$$

Este último en coordenadas originales, $\left(\hat{x}_{u}{ }^{\prime}, \hat{x}^{\prime}\right)^{\prime}=\phi^{-1}(\hat{z}, \beta)$, está dado por:

$$
\begin{aligned}
& \hat{\hat{x}}_{u}=\Gamma_{u} \hat{x}_{u}+K_{u}\left(u-\Delta_{u} \hat{x}_{u}\right), \quad \hat{u}=\Delta_{u} \hat{x}_{u} \\
& \dot{\hat{x}}_{q}=K_{l}\left(s_{o}\right)[y-h(\hat{x}, \beta)] \\
& \dot{\hat{x}}=f\left(\hat{x}, \Delta_{u} \hat{x}_{u}, \beta\right)+G\left(\hat{x}, \hat{x}_{u}, \beta\right)[y-h(\hat{x}, \beta)]+H\left(\hat{x}, \hat{x}_{u}, \beta\right) \hat{x}_{q}, \\
& \hat{y}=h(\hat{x}, \beta), \quad \hat{z}=h_{z}(\hat{x}, \beta)
\end{aligned}
$$


donde $G$ es la ganancia proporcional definida en (3.51), y $\underline{\mathrm{H}}$ es la ganancia integral definida por

$$
H\left(\hat{x}, \hat{x}_{u}, \beta\right)=\left[I_{11}{ }^{\prime}, I_{21}{ }^{\prime}\right]^{\prime} \phi_{I x_{i}}^{-1}\left(\hat{x}, \hat{x}_{u}, \beta\right) \Pi_{o}
$$

Observación 3.8. Ahora el estimador-PI consiste de: (a) un término predictor, que corresponde a una réplica de la planta evaluada con los valores aproximados de los estados y parámetros, (b) un término corrector (forzado por la diferencia entre la medición real y su estimado) formado por un primer término con ganancia proporcional $\left(K_{0}\right)$ para compensar errores rápidos contenidos en la dinámica observable y exo-observable, y un segundo término con ganancia integral $\left(K_{\mathrm{l}}\right)$ para compensar errores lentos debido a errores no observable y errores en los parámetros del modelo.

\subsubsection{Dinámica del error de estimación}

Al error de estimación definido en (3.39) se le agrega el nuevo estado:

$$
e=\left[e_{q}, e_{I}, e_{I I}, e_{u}, e_{b}\right], \quad e_{q}=\hat{z}_{q}-z_{q}
$$

Restando la planta aumentada (3.30) de su estimador-PI (3.64) se obtiene la siguiente dinámica del error de estimación:

$$
\begin{array}{ll}
\dot{e}_{u}=A_{u} e_{u}-\Pi_{u} v(t), & A_{u}=\Gamma_{u}-K_{u} \Delta_{u}, \mu=\Delta_{u} e_{u} \\
\dot{e}_{q}=-K_{I} \Delta_{o} e_{I} & \\
\dot{e}_{I}=A_{o} e_{I}+\Pi_{o}\left[q_{r}\left(e_{I}, e_{u}, t\right)+q_{l}\left(e_{I I}, e_{b}, t\right)+e_{q}\right], A_{o}=\Gamma_{o}-K_{o} \Delta_{o}, v=\Delta_{o} e_{I} \\
\dot{e}_{I I}=\omega\left(e_{I I}, t\right)+q_{I I}\left(e_{I}, e_{I I}, e_{u}, e_{b}, t\right) &
\end{array}
$$

O bien, empleando las matrices aumentadas (y definidas en Apéndice D) $A_{o}^{e}, \Pi_{o}^{e}, y \Delta_{o}^{e}$ :

$$
\begin{array}{lr}
\dot{e}_{u}=A_{u} e_{u}-\Pi_{u} \mathrm{v}(t), & \mu=\Delta_{u} e_{u} \\
\dot{e}_{e}=A_{o}^{e} e_{e}+\Pi_{o}^{e} q_{I}\left(e_{I}, e_{I I}, e_{u}, e_{b}, t\right), \quad e_{e}=\left(e_{I}^{\prime}, e_{q}{ }^{\prime}\right)^{\prime}, & v=\Delta_{o} e_{I} \\
\dot{e}_{I I}=\omega\left(e_{I I}, t\right)+q_{I I}\left(e_{I}, e_{I I}, e_{u}, e_{b}, t\right) &
\end{array}
$$

donde $q_{I}=q_{r}+q_{l}$ y $q_{I I}$ ademâs de ser perturbaciones desvanecientes [(3.60), (3.42)], son L-continuas y acotadas de acuerdo a:

$$
\|v(t)\| \leq \varepsilon_{v}
$$




$$
\begin{gathered}
\left\|q_{I}\left(e_{I}, e_{I I}, e_{u}, \mathrm{v}, e_{b}, t\right)\right\| \leq M_{I}\left\|e_{I}(t)\right\|+M_{I I}\left\|e_{I I}(t)\right\|+M_{u}\left\|e_{u}(t)\right\|+M_{b}\left\|e_{b}\right\| \\
\left\|q_{I I}\left(e_{I}, e_{I I}, e_{u}, e_{b}, t\right)\right\| \leq N_{I}\left\|e_{I}(t)\right\|+N_{u}\left\|e_{u}(t)\right\|+N_{b}\left\|e_{b}\right\| \\
\left(m_{I}, m_{I I}, n_{I}\right)=\lim _{e_{I}, e_{I I}, e_{u}, e_{b} \rightarrow 0}\left(M_{I}, M_{I I}, N_{I}\right)
\end{gathered}
$$

Las ganancias $\left[K_{\mathrm{u}}\left(s_{\mathrm{u}}\right), K_{\mathrm{o}}\left(s_{\mathrm{o}}\right)\right.$ y $\left.K_{\mathrm{I}}\left(s_{\mathrm{o}}\right)\right]$ del estimador-PI (3.65) son ajustadas apropiadamente, de acuerdo a la siguiente forma parametrizada:

$$
\begin{aligned}
& K_{u}\left(s_{u}\right)=d b\left[\left(s_{u} k_{11}^{u}, \ldots, s_{u}^{v_{1}} k_{v_{1} 1}^{u}\right)^{\prime}, \ldots,\left(s_{u} k_{1 p}^{u}, \ldots, s_{u}^{v_{p}} k_{v_{p} p}^{u}\right)^{\prime}\right], \quad s_{u}>0 \\
& K_{o}\left(s_{o}\right)=d b\left[\left(s_{o} k_{11}^{o}, \ldots, s_{o}^{\kappa_{1}} k_{\kappa_{1} 1}^{o}\right)^{\prime}, \ldots,\left(s_{o} k_{1 m}^{o}, \ldots, s_{o}^{\kappa_{m}} k_{\kappa_{m} m}^{o}\right)^{\prime}\right], \quad s_{o}>0 \\
& K_{I}\left(s_{o}\right)=\operatorname{diag}\left[s_{o}^{\mathrm{K}_{1}+1} k_{1}^{I}, \ldots, s_{o}^{\kappa_{m}+1} k_{\kappa_{m}}^{I}\right]
\end{aligned}
$$

con entradas de referencia $\mathrm{K}_{\mathrm{u}}(1), \mathrm{K}_{\mathrm{o}}(1)$, y $\mathrm{K}_{\mathrm{I}}(1)$, tales que hacen estable la dinámica (LNPA) del error de salida $\left[\mu=\Delta_{u} e_{u}:=\hat{u}-u\right.$, y $\quad v=\Delta_{o} e_{I}:=\hat{y}-y$ de los sistemas dinámicos (3.69)]:

$$
\begin{array}{ll}
\mu_{i}^{\left(v_{i}\right)}+k_{1 i}^{u} \mu_{i}^{\left(v_{i}-1\right)}+\ldots+k_{v_{i} i}^{u} \mu_{i}=0, & 1 \leq i \leq p ; \quad \mu_{i}=\hat{u}_{i}-u_{i} \\
v_{i}^{\left(\kappa_{i}+1\right)}+k_{1 i}^{o} v_{i}^{\left(\kappa_{i}\right)}+\ldots+k_{\kappa_{i} i}^{o} \dot{v}_{i}+k_{i}^{I} v_{i}=0, & 1 \leq i \leq m ; \quad v_{i}=\hat{y}_{i}-y_{i}
\end{array}
$$

tal que las matrices $A_{\mathrm{u}}\left(s_{\mathrm{u}}\right)$ y $A_{\mathrm{o}}^{\mathrm{e}}\left(s_{\mathrm{o}}\right)$ cumplen con las siguientes desigualdades:

$$
\begin{aligned}
& \left\|e^{A_{u}\left(s_{u}\right) t}\right\| \leq a_{u} e^{-s_{u} \lambda_{u} t}, \quad t \geq 0 \\
& \left\|e^{A_{o}^{e}\left(s_{o}\right) t}\right\| \leq a_{o}^{e} e^{-s_{o} \lambda_{o}^{t} t}, \quad t \geq 0
\end{aligned}
$$

donde $\left(a_{u}, \lambda_{u}\right)$, y $\left(a_{o}^{e}, \lambda_{o}^{e}\right)$ están determinados por las ganancias de referencia $\mathrm{K}_{\mathrm{u}}(1), \mathrm{K}_{\mathrm{o}}(1), \mathrm{y} \mathrm{K}_{\mathrm{I}}(1)$, y son independientes de los parámetros $\left(s_{\mathrm{u}}, s_{\mathrm{o}}\right)$.

\subsubsection{Convergencia}

Como se estableció para el estimador-P, la dinámica del error de estimación (3.69) para el estimador-PI es RE-estable sin las perturbaciones $\left(u, q_{l}, q_{I I}\right)$. Las condiciones para la convergencia-RE, asi como la sinopsis de la construcción del estimador-PI, se dan el siguiente teorema.

Teorema 2 (Prueba en Apéndice E). Sea la trayectoria $x(t)$ de la planta (3.1) REestimable, de acuerdo a la Definición 2, y sean las ganancias de referencia 
$\mathrm{K}_{\mathrm{u}}(1), \mathrm{K}_{\mathrm{o}}(1)$ y $\mathrm{K}_{\mathrm{I}}(1)$, que hacen estable la dinámica LNPA del error de salida (3.75). Entonces, el estimador-PI

$$
\begin{aligned}
& \dot{\hat{x}}_{u}=\Gamma_{u} \hat{x}_{u}+K_{u}\left(u-\Delta_{u} \hat{x}_{u}\right), \quad \hat{u}=\Delta_{u} \hat{x}_{u} \\
& \dot{\hat{x}}_{q}=K_{I}\left(s_{o}\right)[y-h(\hat{x}, \beta)] \\
& \dot{\hat{x}}=f\left(\hat{x}, \Delta_{u} \hat{x}_{u}, \beta\right)+G\left(\hat{x}, \hat{x}_{u}, \beta\right)[y-h(\hat{x}, \beta)]+H\left(\hat{x}, \hat{x}_{u}, \beta\right) \hat{x}_{q}, \\
& \hat{y}=h(\hat{x}, \beta), \quad \hat{z}=h_{z}(\hat{x}, \beta)
\end{aligned}
$$

con ganancias parametrizadas $\mathrm{K}_{\mathrm{u}}\left(s_{\mathrm{u}}\right), \mathrm{K}_{\mathrm{o}}\left(s_{\mathrm{o}}\right)$ y $\mathrm{K}_{\mathrm{I}}\left(s_{\mathrm{o}}\right)$ (3.74), y las ganancias no lineales

$$
\begin{gathered}
G\left(\hat{x}, \hat{x}_{u}, \beta\right)=\left[I_{11}{ }^{\prime}, I_{21}{ }^{\prime}\right]^{\prime} \phi_{I x_{l}}^{-1}\left(\hat{x}, \hat{x}_{u}, \beta\right) K_{o}\left(s_{o}\right) \\
H\left(\hat{x}, \hat{x}_{u}, \beta\right)=\left[I_{11}{ }^{\prime}, I_{21}{ }^{\prime}\right]^{\prime} \phi_{I x_{l}}^{-1}\left(\hat{x}, \hat{x}_{u}, \beta\right) \Pi_{o}
\end{gathered}
$$

genera una trayectoria $\hat{x}(t)$ que RE-converge a $x(t)$, si el parámetro $s_{0}$ es suficientemente grande tal que

$$
\begin{array}{ll}
s_{o} \lambda_{o}^{e}>a_{o}^{e} m_{I} & \text { si } \kappa=n, \quad \text { o } \\
s_{o} \lambda_{o}^{e}>a_{o}^{e} m_{I}+a_{o}^{e} c_{x}^{\phi}\left(a_{x} / \lambda_{I I}\right) m_{I I} n_{I} & \text { si } \kappa<n
\end{array}
$$

donde $\left(\mathrm{c}_{\mathrm{x}}^{\phi}, a_{\mathrm{x}}, \lambda_{\mathrm{II}}, m_{\mathrm{l}}, m_{\mathrm{II}}, n_{\mathrm{I}}\right)$ están definidas en el Teorema $1, \mathrm{y}\left(a_{o}^{e}, \lambda_{o}^{e}\right)$ están definidas en (3.77).

Como puede verse la condición de convergencia del estimador-PI (3.81) es similar a la del estimador-P (3.52), con única diferencia en el par $\left(a_{o}^{e}, \lambda_{o}^{e}\right)$ y $\left(a_{0}, \lambda_{0}\right)$. Esto debido a que las dinámicas del error de estimación [ec. (3.40) para el estimador-P y ec. (3.69) para el estimador-PI) tienen la misma estructura [únicamente difieren en el nuevo estado aumentado $\left.e_{e}=\left(e_{I}{ }^{\prime}, e_{q}{ }^{\prime}\right)^{\prime}\right]$. De forma similar, en el siguiente corolario del Teorema 2 se presenta la caracterización del estimador-PI RE-convergente.

Corolario del Teorema 2. Sea la trayectoria $x(t)$ de la planta (3.1) RE-estimable, de acuerdo a la Definición 2, y sea la condición de convergencia (3.81) del Teorema 2 que se cumple con $s_{\mathrm{o}}$ y $s_{\mathrm{u}}$ tal que

$$
\begin{array}{ll}
l_{u}\left(s_{u}\right)>L_{I}^{e}\left(s_{o}\right) & \text { si } \kappa<n, \text { o } \\
l_{u}\left(s_{u}\right)>L_{I}^{e}\left(s_{o}\right)>L_{l I} & \text { si } \kappa=n
\end{array}
$$

con factores de decaimiento dados por 


$$
l_{u}=s_{u} \lambda_{u}, \quad L_{I}^{e}=s_{o} \lambda_{o}^{e}-a_{o}^{e} M_{I} \quad\left\{\begin{array}{l}
L_{x}^{e}=L_{I}^{e} \text { si } \kappa<n \\
L_{x}^{e}>L_{I I} \text { si } \kappa=n
\end{array}\right.
$$

Entonces, los estados, las entradas y las salidas estimados del estimador PI, convergen-RE de acuerdo a las desigualdades (3.54) del corolario, reemplazando $\left(a_{o}, \lambda_{o}\right)(3.49)$ y $\left(A_{a}, L_{x}\right)$ (E.57) por $\left(a_{o}^{e}, \lambda_{o}^{e}\right)(3.77)$ y $\left(A_{a}^{e}, L_{x}^{e}\right)(\mathrm{E} .82)$, respectivamente.

Observación 3.9. La interpretación de la caracterización de la convergencia del estimador-PI es similar a la explicada en el corolario del Teorema 1. Comparando con el estimador-P, y empleando el mismo esquema de sintonización (que se verá en la sección 3.8), puede verse que en los corolarios: $a_{o}^{e}>a_{o}, A_{a}^{e}>A_{a}, L_{l}^{e}>L_{l}$, y $L_{x}^{e}>L_{x}$. Lo cual significa que el diseño-PI genera sesgos en los estados y salidas más pequeños que el diseño-P, pero el costo es una convergencia más lenta y oscilatoria (esto último porque el estimador-PI tiene dinámicas del error de estimación con un orden mayor a las correspondientes al diseño-P). Por lo que para implementar un diseño, se debe evaluar cuando es apropiada la acción integral, así como se hace en el diseño de controladores lineales.

\subsection{Resumen del diseño del estimador y sus grados de libertad}

Hasta aquî se ha establecido el diseño del estimador geométrico no lineal (P o PI). En la Definición 2 se establecen condiciones necesarias para la existencia de solución al problema de estimación de una trayectoria de la planta.

El Teorema 1 y el Teorema 2 proporcionan la construcción sistemática del estimador $\mathrm{P}$ o PI, respectivamente. Asimismo estos teoremas dan un procedimiento de sintonización sistemático y simple, donde la selección de las ganancias de referencia $\left[K_{\mathrm{u}}(1), K_{\mathrm{o}}(1)\right.$ y $\left.K_{\mathrm{I}}(1)\right]$ puede hacerse fácilmente mediante diversas técnicas de sintonización reportadas en literatura (Stephanopoulos, 1984; Ogata, 1980) para sistemas lineales (las cuales se basan en la asignación de polos). En la sección 3.8 y en el Apéndice $F$ se presentan las técnicas de sintonización empleadas y recomendadas para el estimador propuesto.

Los corolarios de los teoremas muestran el papel y el compromiso existente entre la estructura de estimabilidad, las ganancias parametrizadas, la velocidad de convergencia, el tamaño del sesgo, el tamaño de la vecindad de convergencia, la 
convergencia alcanzable, y el grado de robustez. Es importante mencionar que la convergencia- $\mathrm{RE}$ del esquema de estimación aquí propuesto es un resultado local, en el sentido de que las condiciones de convergencia ((3.52) y (3.81)) son válidas para una vecindad de la planta (de tamaño y forma no definido, pero pequeña). En principio, la metodologia de convergencia presentada en este trabajo es extendible al caso no local (Isidori, 1995). En trabajos de investigación recientes (Alvarez et al., 1999; 2000) ya se está abordando el caso no local, como una continuación al presente trabajo de investigación doctoral; donde las ganancias del estimador pueden ser ajustadas para asegurar la convergencia para un conjunto compacto de estados iniciales - entradas - parámetros del modelo.

En el corolario del Teorema 1, el factor de amplitud $a_{\mathrm{x}}$ [que determina el tamaño de la parte transitoria del error en estados, $\hat{x}(t)-x(t)]$ es proporcional al número de condición $c_{\phi}^{x}$ [definido en (3.33)] del mapa de estimabilidad $\phi$ a lo largo de la trayectoria de la planta. En el caso de un detector $(\kappa>n)$, el margen de desestabilización es proporcional a $c_{\phi}^{x}$ [ver ecuaciones (3.52) y (3.81)]. Por lo tanto, un mapa $\phi$ mal condicionado ( $c_{\phi}^{x}$ grande) hará que la convergencia del estimador sea débil (esto es, con poca tolerancia a errores en estados iniciales - entradas parámetros del modelo). Por ello, el estimador deberá diseñarse de tal forma que se logre un compromiso entre el desempeño dinámico y la robustez.

De los teoremas y corolarios, en conjunción con la propiedad de estimabilidad-RE establecida en la Definición 2, el funcionamiento del estimador geométrico [P en ec. (3.50) o PI en ec. (3.78)] depende del diseño seleccionado, el cual tiene los siguientes grados de libertad:

I. La estructura de estimabilidad $S$ (3.19), la cual depende a su vez de dos factores a escoger: el vector de estimabilidad $k=\left(\kappa_{1}, \ldots, \kappa_{\mathrm{m}}\right)$ y la partición de los mapas observables y no observables $\left(x_{\mathrm{I}}, x_{\mathrm{II}}\right)$.

II. La sintonización del exo-observador y del estimador, en la cual se requiere fijar las ganancias de referencia $\left[K_{\mathrm{u}}(1), K_{\mathrm{o}}(1)\right]$ y $K_{\mathrm{I}}(1)$ (en caso del estimador-PI) y los parámetros de ajuste $\left(s_{\mathrm{u}}, s_{\mathrm{o}}\right)$ para la convergencia, que en conjunción determinan las ganancias parametrizadas $K_{\mathrm{u}}\left(s_{\mathrm{u}}\right), K_{\mathrm{o}}\left(s_{\mathrm{o}}\right)$ y $K_{\mathrm{I}}\left(s_{\mathrm{o}}\right)$.

El criterio de selección de cada uno de estos factores, y la forma en que afecta cada uno de ellos al funcionamiento del estimador se discuten en las siguientes dos secciones. 


\subsection{Estructura y medida de estimabilidad}

La selección de la estructura de estimabilidad constituye la parte fundamental del diseño del estimador, ya que dependiendo del grado de estimabilidad $\left(\kappa=\kappa_{1}+\ldots+\right.$ $\kappa_{\mathrm{m}}$ ) será: el número de ganancias a ajustar (número de ganancias $=v+\kappa \dot{o} v+\kappa+m$, para el estimador-P y PI, respectivamente), y las caracteristicas de convergencia (velocidad, amplitud, sesgo, y robustez) de la trayectoria estimada de la planta.

Algunas consideraciones para elegir el vector de estimabilidad $k=\left(\kappa_{1}, \ldots, \kappa_{\mathrm{m}}\right)$ y la partición de estados $\left(x_{\mathrm{I}}, x_{\mathrm{II}}\right)$ son las siguientes:

A. Para alcanzar la velocidad de convergencia más rápida en un diseño nominal (i.e., sin errores de modelado), el grado de estimabilidad debe ser lo más grande posible. Si existen errores en los parámetros del modelo y la planta es sensible a ello, entonces el grado de estimabilidad debe reducirse (siempre y cuando la trayectoria no observable sea RE-estable), para prevenir una propagación de errores numéricos a través de las derivadas parciales de alto orden de los mapas $f$ y $h$ en los mapas $\phi$ y $\varphi$ de la propiedad de estimabilidad de la trayectoria de la planta Por lo tanto: altos índices de observabilidad favorecen el desempeño nominal del estimador, pero es desfavorable para su robustez.

B. Por otra parte, el funcionamiento robusto del estimador se ve afectado también por la selección de la partición $\left(x_{\mathrm{I}}, x_{\mathrm{II}}\right)$, y su papel en la estructura de estimabilidad es como sigue: bajos indices de observabilidad $\left(\operatorname{dim}\left(x_{\mathrm{I}}\right)\right.$ pequeña) favorecen la robustez de la dinámica observable, pero desfavorece tanto la velocidad de reconstrucción de los estados como la robustez de la dinámica no observable. Esto debido a que con una dimensión de $x_{I I}$ grande existe mayor posibilidad de inestabilidad (dependiendo esto también de los estados seleccionados en cada parte de $x_{l}$ y $\left.x_{l l}\right)$, asimismo la tolerancia a errores de modelado es menor ya que el estimador sólo se puede atenuar los errores que se propagan en la parte observable.

Observación 3.10. Retomando el ejemplo definido en la Observación 3.3 (analizado en la Observación 3.4), se concluyó que las estructuras de estimabilidad $S$ que cumplen la propiedad de estimabilidad (Definición 2) son: $S_{2}=\left\{(1,1),(M, T)^{\prime}\right\}, S_{4}=$ $\left\{(2,1),(M, I, T)^{\prime}\right\}$, y $S_{5}=\left\{(1,2),(M, I, T)^{\prime}\right\}$. De acuerdo a las hipótesis anteriores (A y B): la implementación de las estructuras observables $S_{4}$ y $S_{5}$ deberán generar respuestas más rápidas que la estructura detectable $S_{2}$, además que como el mapa $\phi_{1}$ de $S_{4}$ y $S_{5}$ contiene sólo una primera derivada entonces estas estructuras serán robustas (esto es, con tolerancia ante errores de modelado y ante propagación del error). En 
conclusión, para el ejemplo definido en la Observación 3.3, se tiene originalmente 5 posibles estructuras, sin embargo sólo 3 estructuras cumplen la propiedad de estimabilidad, y para propósitos de obtener un buen desempeño (rapidez de convergencia y robustez) se tiene a priori 2 posibles estructuras: $S_{4}=\left\{(2,1),(M, I, T)^{\prime}\right\}$, y $S_{5}=\left\{(1,2),(M, I, T)^{\prime}\right\}$. No obstante, para procesos más complejos (como es el caso de estudio del reactor continuo de copolimerización en solución) puede ser que aún con estos criterios se tenga un gran número de estructuras candidatas. Para ello, enseguida se definirán medidas de observabilidad que permitan diferenciar y elegir entre un conjunto grande de estructuras candidatas.

Tomando en cuenta las consideraciones anteriores, se observa que la selección de la estructura de estimabilidad $S$ no es trivial (aún más cuando $n$ es grande, ya que la cardinalidad de $S$ aumenta).En literatura se encuentra reportado que para abordar el problema de selección de la estructura de medición de un estimador lineal (Johnson, 1969; Muller y Weber, 1977; Moore, 1981) se han introducido medidas de la propiedad de estimabilidad. Estas ideas han sido aplicadas para el diseño de estructuras de medición de procesos químicos (Kumar y Seinfeld, 1978; Rogmanoli et al., 1981; Alvarez et al., 1981; Lau et al., 1985; Waldraff et al., 1998). Sin embargo su aplicación ha sido excluida debido a la ausencia de fundamentos en estimación no lineal. Estas consideraciones sugieren que debiera usarse una metodologia, dentro del marco de estimación geométrico no lineal, para seleccionar la estructura de estimabilidad en el diseño de un estimador no lineal.

Con base en lo anterior, en el siguiente lema se reformula la propiedad de estimabilidad-RE (Definición 2) en têrminos de condiciones suficientes, con funciones medibles y manejables númerica-cuantitativamente. $Y$ enseguida se compara con las medidas existentes para sistemas observables lineales.

Sea $c(A)$ y $s(A)$, el número de condición (equivalente a la medida del mal condicionamiento) y la medida de sensibilidad de una matriz rectangular $A$,

$$
c(A)=\frac{\sigma^{*}(A)}{\sigma_{*}(A)}, \quad s(A)=\frac{1}{\sigma^{*}(A)}
$$

donde $\sigma_{*}(A)$ y $\sigma^{*}(A)$ son los valores singulares minimo y máximo de la matriz $A$. Las funciones de $x$-condicionamiento $\left[c_{\mathrm{x}}^{\alpha}(t)\right], u$-sensibilidad $\left[s_{\mathrm{u}}^{\alpha}(t)\right]$, y $b$-sensibilidad $\left[s_{\mathrm{b}}^{\alpha}(t)\right]$ de un mapa $\alpha(x, u, b)$ continuamente diferenciable, a lo largo del conjunto trayectoria parámetro $\{x(t), u(t), b\}$, están dados por:

$$
\left[c_{x}^{\alpha}(t), s_{u}^{\alpha}(t), s_{b}^{\alpha}(t)\right]=\left[c\left(\frac{\partial \alpha}{\partial x}\right), s\left(\frac{\partial \alpha}{\partial u}\right), s\left(\frac{\partial \alpha}{\partial b}\right)\right][x(t), u(t), b]
$$


Lema 1 (Estimabilidad robusta-exponencial). La trayectoria $x(t)(3.3)$ de la planta $n$ dimensional (3.1) es $\mathrm{RE}$ (robustamente exponencialmente)-estimable si existe una estructura de estimabilidad $S$ (3.19) tal que, a lo largo de la evolución de la planta $\mathrm{E}(t)(3.2)$ :

(i) El mapa $\phi_{\mathrm{I}}(x, \mathcal{U}, b)(3.11)$ tiene funciones $x_{\mathrm{I}}$-condicionamiento y $(\mathcal{U}, b)$-sensibilidad acotadas.

(ii) El mapa $\varphi(x, \mathcal{U}, v, b)(3.12)$ tiene un conjunto de funciones $(x, \mathcal{U}, v, b)$-sensibilidad acotado.

(iii) El mapa $f_{I I}\left[x_{I I}, \mathcal{U}(t), y(t), b\right]$ de la dinámica no observable (3.16) tiene una función de margen de estabilidad $l_{s}(t)(2.15)$ estrictamente positiva, así como funciones $x_{\mathrm{II}}$-condicionamiento y $(\mathcal{U}, y, b)$-sensibilidad acotadas.

Observación 3.11. Las condiciones del Lema 1 son equivalentes a las condiciones de la Definición 2. Simplemente se han reformulado, empleado herramientas matemáticas. Cabe señalar que la función $x_{\mathrm{I}}$-condicionamiento del mapa $\phi_{\mathrm{I}}$ (condición (i)) corresponde al número de $c_{x}^{\phi}$ involucrado en la condición de convergencia (3.52) del estimador, el cual a su vez está determinado por la estructura de estimabilidad $S$ y la trayectoria de operación $x(t)$ seleccionadas. De aquí nuevamente la importancia de seleccionar adecuadamente la estructura de estimabilidad, y de realizar una sintonización adecuada para vencer los potenciales de desestabilización (definidos en la Observación 3.6).

Si el sistema (3.1) es lineal, invariante en tiempo, y completamente observable (esto es, $\mathrm{\kappa}=n$ y $\left.x=x_{1}\right)$ :

$$
\dot{x}=A x, \quad y=C x, \quad C=\left[c_{1}{ }^{\prime}, \ldots, c_{m}{ }^{\prime}\right]^{\prime}
$$

entonces las condiciones (ii) y (iii) del Lema 1 se cumplen trivialmente, y la condición (i) se convierte en la condición del rango de observabilidad $(O$ es la matriz de observabilidad)

$$
\text { rango }(O)=n, \quad O^{\prime}=\left[c_{1}^{\prime}, \ldots,\left(c_{1} A^{\kappa_{1}}\right)^{\prime} ; \ldots ; c_{m}^{\prime}, \ldots,\left(c_{m} A^{\kappa_{m}}\right)^{\prime}\right]^{\prime}, \quad \kappa_{1}+\ldots+\kappa_{m}=n
$$

Siendo en este caso, $\sigma_{*}(O)$ una medida del grado de observabilidad (Johnson, 1969; Muller y Weber, 1972). Esta medida ha sido aplicada para diseñar y seleccionar configuraciones de salidas en reactores tubulares (Romagnoli et al., 1981; Alvarez et al. 1981; Waldraff et al., 1998), con la base de una aproximación lineal del modelo del proceso. Aquí, la estructura de estimabilidad $S$ que es una selección del 
diseñador (quien fija la partición y acomodo de las dinámicas observable y no observable), y las medidas del Lema 1 son relacionadas a la cantidad de información robustamente disponible de la parte observable de la planta [condición (i)], la intensidad y sensibilidad del acoplamiento entre las dinámicas observable y no observable [condición (ii)], y la robustez de la dinámica no observable [condición (iii)].

\subsection{Sintonización}

La sintonización o ajuste de las ganancias es uno de los aspectos importantes en el diseño del estimador. Ya que a pesar de tener un diseño (P ó PI) RE-convergente y una construcción sistemática del estimador geométrico no lineal, existen conflictos y compromisos inherentes al proceso. Por ejemplo, existe un compromiso entre la velocidad de convergencia y el grado de robustez para mantener un desempeño del estimador aceptable. Describiendo "desempeño aceptable" a las siguientes características: estabilidad, respuesta rápida y suave (poco oscilatoria), sin (o pequeño) sesgo, mínima propagación de error numérico y/o ruido de medición, poca sensibilidad ante cambios en las condiciones de operación $\mathrm{y}$ ante errores de modelado, etc.

El ajuste de las ganancias de referencia, tanto para el exo-observador $\left[K_{\mathrm{u}}(1)\right]$ como para el estimador de estados $\left[K_{0}(1)\right.$, o el par $\left.\left\{K_{0}(1), K_{\mathrm{I}}(1)\right\}\right]$, puede realizarse mediante procedimientos similares a las técnicas de sintonización para diseños de control lineal UEUS (una entrada-una salida), ya que las ganancias de referencia son los coeficientes de la dinámica LNPA del error de salida estimación [ec. (3.47) para estimador-P y ec. (3.75) para estimador-PI].

Existen diversas técnicas de sintonización en la teoría de control lineal convencional (Stephanopoulos, 1984): criterios de prueba y error (lo cual resulta tedioso), reglas semi-empíricas (las cuales se establecen en la práctica), criterios simples (como el criterio de un cuarto de la razón de decaimiento), criterios de desempeño del error integral en tiempo (tal como ISE, Integral of the square error, IAE, Integral of the absolute value of the error, e ITAE, Integral of time-weight absolute error).

Sin embargo, existe cierta ambigüedad en la selección del método de sintonización, debido a que algunas técnicas suprimen errores grandes o pequeños, o favorecen respuestas oscilatorias, o bien dependen de la respuesta ante un cambio (escalón, rampa, etc.) en las entradas. Consecuentemente, lo deseable es tener un procedimiento sistemático de sintonización, asi como valores iniciales buenos para las ganancias de referencia. 
En las siguientes dos subsecciones se presentan las técnicas de sintonización propuestas para el exo-observador (ajuste de $K_{\mathrm{u}}$ ) y para el estimador [ajuste de $K_{\mathrm{o}}(1)$, o el par $\left\{K_{0}(1), K_{\mathrm{I}}(1)\right]$. Las técnicas propuestas tienen como fundamento la asignación de polos en el plano complejo, sin embargo, la asignación se hace de forma diferente para el exo-observador y para el estimador, ya que la implementación de cada uno de estos tienen propósitos particulares. El objetivo principal del exo-observador es el manejo (filtrado) del ruido en las entradas medidas $(u)$, mientras que el del estimador es obtener estimados RE-convergentes de los estados $(x)$.

Cabe mencionar que en la técnica de localización de polos, para que un sistema dinámico lineal (de coeficientes constantes) sea estable debe contener sus polos $\left(\lambda_{i}=\right.$ $a \pm b j, i=1, \ldots, \mathrm{n})$ dentro del lado izquierdo del eje imaginario en el plano complejo (i.e, $a<0$ ). Asimismo, el desempeño de la respuesta dinámica depende de la localización de los polos (ver Figura 3.2): la velocidad de convergencia es mayor si la parte real de $\lambda_{i}$ es más pequeña (más lejos del eje imaginario, es decir, si $a<<0$ ), y el desempeño tiende a ser más oscilatorio si el valor absoluto de la parte imaginaria es más grande (o bien más lejos del eje real, es decir, si $|b|>>0$ ).

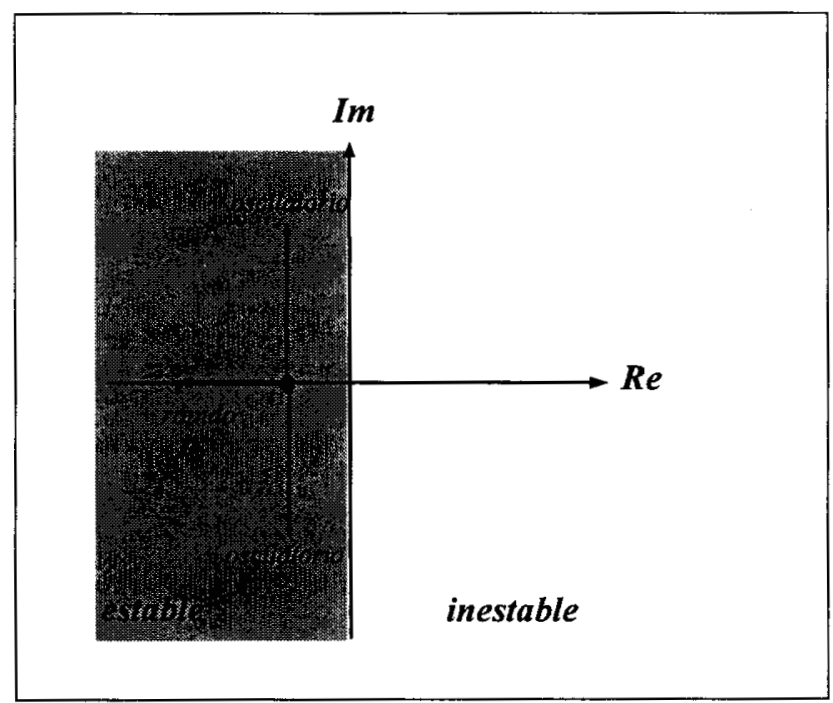

Figura 3.2 Localización de polos en el plano complejo 


\subsubsection{Sintonización del exo-observador}

Debido a que el propósito central del exo-observador es el filtrado del ruido en las entradas medidas, sus ganancias pueden ser ajustadas mediante técnicas estándar de filtrado óptimo estocástico lineal (Gelb, 1974; Kwakernaak y Sivan, 1972).

Sean $v_{\mathrm{i}} \mathrm{y} w_{1}$ procesos estocásticos gaussianos, con media cero $\mathrm{y}$ sin correlación temporal (i.e. ruido blanco) para errores en el modelo y ruido en las mediciones, respectivamente; con intensidades $q_{\mathrm{i}}$ y $r_{\mathrm{i}}$ :

$$
v_{i} \sim N\left[0, q_{i}\right], \quad w_{i}-N\left[0, r_{i}\right]
$$

entonces la versión estocástica del exo-sistema (3.22) es $\left(\Gamma_{i}^{u}, \Pi_{i}^{u}, \mathrm{y} \delta_{i}^{u}\right.$ se definen en Apéndice D)

$$
\begin{aligned}
& \dot{x}_{i}^{u}=\Gamma_{i}^{u} x_{i}^{u}+\Pi_{i}^{u} v_{i}(t) \\
& u_{i}=\delta_{i}^{u} x_{i}^{u}+w_{i}(t), \quad 1 \leq i \leq p
\end{aligned}
$$

Y para el cual, el filtro óptimo (Kwakernaak y Sivan, 1972) está dado por:

$$
\begin{array}{cc}
\dot{\hat{x}}_{i}^{u}=\Gamma_{i}^{u} \hat{x}_{i}^{u}+\Pi_{i}^{u} v_{i}(t)+K_{i}^{u}\left(u_{i}-\delta_{i}^{u} \hat{x}_{i}^{u}\right) \\
K_{i}^{u}=r_{i}^{-1} \Sigma_{i} \delta_{i}^{u}{ }^{\prime}, & 1 \leq i \leq p
\end{array}
$$

donde la matriz de covarianza $\Sigma_{\mathrm{i}}$ (de dimensión $v_{\mathrm{i}} \mathrm{x} \mathrm{v}_{\mathrm{i}}$ ) está determinada por la solución de la ecuación de Riccati (en estado estacionario, esto es, $\dot{\Sigma}=0$ ):

$$
\Sigma_{i} \Gamma_{i}^{u}+\Gamma_{i}^{u} \Sigma_{i}+q_{i} \Pi_{i}^{u} \Pi_{i}^{u \prime}-r_{i}^{-1} \Sigma_{i} \delta_{i}^{u \prime} \delta_{i}^{u} \Sigma_{i}=0, \quad 1 \leq i \leq p
$$

Fijando el cociente de referencia ruido del modelo - ruido de medición $\left(q_{i}^{r} / r_{i}^{r}\right)$, y resolviendo para $K_{i}^{u}$ en términos de $\omega_{i}^{u}$ y $s_{\mathrm{u}}$, los cuales se definen como:

$$
\omega_{i}^{u}=\left(\frac{q_{i}^{r}}{r_{i}^{r}}\right)^{\frac{1}{2 v_{i}}}, \quad s_{u} \omega_{i}^{u}=\left(\frac{q_{i}}{r_{i}}\right)^{\frac{1}{2 v_{i}}}
$$

tal que la ganancia $K_{i}^{u}\left(s_{u}\right)$ corresponde al i-ésimo vector de la matriz parametrizada $K_{\mathrm{u}}\left(s_{\mathrm{u}}\right)$ [en (3.46) o (3.74)], con ganancia de referencia $K_{i}^{u}(1)$ determinada por $\omega_{i}^{u}$ y dada en la Tabla 3.1 (ver Apéndice F para detalles del procedimiento de obtención de las ganancias de referencia dadas en esta tabla).

La dinámica correspondiente al i-ésimo error de salida del exo-observador $\left[\mu_{i}=\hat{u}_{i}-u_{i}\right.$ en (3.75)] tiene una configuración de polos de tipo Butterworth (D'azzo y Houpis, 
1995). Este tipo de configuración tiene una localización de polos como se muestra en la Figura 3.3, y cuya interpretación es como sigue (ver detalle de la localización de polos en el Apéndice F):

(i) $\omega_{i}^{u}$ está referida a una frecuencia caracteristica de referencia $\left(\omega_{i}^{u} \approx 1 / \tau_{c}\right.$, donde $\tau_{c}$ es un tiempo característico),

(ii) al trazar un círculo con radio $s_{u} \omega_{i}^{u}$ y centro en el origen del plano complejo, los $v_{\mathrm{i}}$ polos se localizan en distancia $2 v_{\mathrm{i}}$-equidistantes a lo largo del semicirculo estable, o bien con ángulos dados por:

$$
\begin{array}{ll} 
\pm \frac{\left(l+\frac{1}{2}\right) \pi}{v_{i}}, & l=0,1, \ldots, \frac{v_{i}}{2}-1 \quad \text { si } v_{i} \text { es par } \\
\pm \frac{l \pi}{v_{i}}, & l=0,1, \ldots, \frac{v_{i}-1}{2} \quad \text { si } v_{i} \text { es impar }
\end{array}
$$

De tal forma que existe una relación entre el filtro óptimo estocástico y la estructura de polos de Butterworth, lo cual es un hecho conocido en el filtrado lineal de Kalman (Kwakernaak y Sivan, 1972): cuando las ganancias óptimas del filtro de Kalman son evaluadas en estado estacionario, se obtiene la configuración de Butterworth.

Por otra parte, la dinámica del i-ésimo error de salida del exo-observador (3.75) además de tener una configuración de tipo Butterworth, tiene una estructura y localización de polos semejante a los polinomios obtenidos y reportados para el criterio ITAE (los polos coinciden exactamente para $v_{i}=1,2$; mientras que para $v_{i}>2$ se obtiene polos muy cercanos; ver Apéndice F). Por lo que éste último criterio también sería válido y adecuado para la sintonización del exo-observador. Cabe señalar, que el criterio de ITAE en la teoria de control lineal convencional (Stephanopoulos, 1984) es recomendado para suprimir errores que persisten por periodos de tiempo largos [que corresponde al caso de $v_{i}(t)$ y $w_{\mathrm{i}}(t)$ ].

Tabla 3.1 Ganancias de referencia para el exo-observador

\begin{tabular}{|c|c|}
\hline 1 & $\omega_{i}^{u}$ \\
\hline 2 & $1.4 \omega_{i}^{u},\left(\omega_{i}^{u}\right)^{2}$ \\
\hline 3 & $2 \omega_{i}^{u}, 2\left(\omega_{i}^{u}\right)^{2},\left(\omega_{i}^{u}\right)^{3}$ \\
\hline 4 & $2.6 \omega_{i}^{u}, 3.4\left(\omega_{i}^{u}\right)^{2}, 2.6\left(\omega_{i}^{u}\right)^{3},\left(\omega_{i}^{u}\right)^{4}$ \\
\hline 5 & $3.24 \omega_{i}^{u}, 5.24\left(\omega_{i}^{u}\right)^{2}, 5.24\left(\omega_{i}^{u}\right)^{3}, 3.24\left(\omega_{i}^{u}\right)^{4},\left(\omega_{i}^{u}\right)^{5}$ \\
\hline
\end{tabular}




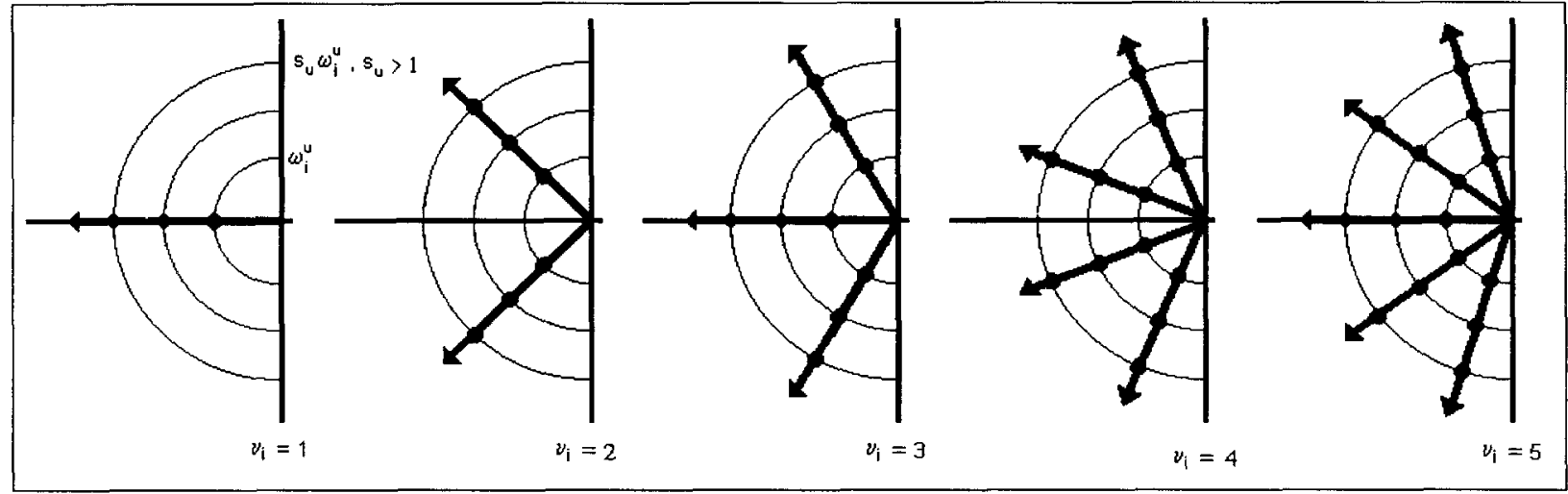

Figura 3.3 Localización de polos para el exo-observador

\subsubsection{Sintonización del estimador}

Para que la condición de convergencia [(3.52) o (3.81)] se cumple fácilmente, si el error no lineal $q_{1}$ en la dinámica del error observable $[(3.40)$ o (3.69)] es suficientemente pequeño; de tal forma que el estimador no sea divergente, y las matrices de ganancias, $K_{\mathrm{o}} \circ\left(K_{\mathrm{o}}, K_{\mathrm{I}}\right)$, puedan ajustarse con un método sistemático de asignación de polos (similar a la sección anterior), en principio, se pudiera emplear el tipo de sintonización de un FEK (filtro extendido de Kalman) no lineal, aunque pudieran aparecer el mismo tipo de limitantes en su implementación (como son los problemas de divergencia, por carecer de un criterio a priori de convergencia) que se han reportado en la literatura (ver Capitulo 1). Por otro lado, para índices de observabilidad grandes (suponiendo mayor de dos), la sintonización (orientada a ruido) del tipo Butterworth siempre coloca un par de polos complejos cerca del eje imaginario, con una amplitud que crece con el parámetro $s_{0}$, lo cual significa tendencia hacia la divergencia o convergencia con poca robustez (ver explicación de esta aserción en el Apéndice F). Para hacer frente a este problema, se propone una estrategia sistemática de asignación de polos para el estimador de la planta.

Reescribiendo la dinámica de referencia LPNA del i-ésimo error de salida [ $v_{\mathrm{i}}$ en (3.47) o (3.75)]:

$$
v_{i}^{\left(n_{i}\right)}+k_{1}^{i} v_{i}^{\left(n_{i}-1\right)}+\ldots+k_{n_{i}}^{i} v_{i}=0, \quad \begin{cases}n_{i}=\kappa_{i} & \text { estimador }-\mathrm{P} \\ n_{i}=\kappa_{i}+1 & \text { estimador }-\mathrm{PI}\end{cases}
$$

cuyo polinomio caracteristico 


$$
\left(\gamma_{i}\right)^{n_{i}}+k_{1}^{i}\left(\gamma_{i}\right)^{n_{i}-1}+\ldots+k_{n_{i}}^{i}=\prod_{l=1}^{n_{i}}\left(\gamma_{i}-\gamma_{l}^{i}\right)=0
$$

tiene $n_{\mathrm{i}}$ polos de referencia caracteristicos $\left(\gamma_{1}^{\mathrm{i}}, \ldots, \gamma_{\mathrm{n}_{\mathrm{i}}}^{\mathrm{i}}\right)$, los cuales se determinan de acuerdo a los siguientes criterios orientados para obtener robustez (y no para eliminar el ruido, como es el caso del exo-observador):

1. el número de condición (i.e., la razón entre el módulo del polo más rápido entre el módulo del polo más lento) debe ser uno, con la finalidad de amortiguar el efecto de la perturbación $q_{\mathrm{I}}$ en la dinámica del error observable [(3.40) o (3.69)].

2. Cada polo debe tener un factor de amortiguamiento $\left(\xi_{i}\right)$ suficientemente grande para evitar un comportamiento excesivamente oscilatorio (ver Apéndice F).

Si $n_{\mathrm{i}}$ es un número par, la dinámica de referencia del error de salida (3.91) tiene $n_{\mathrm{i}} / 2$ pares de polos complejos con frecuencias caracteristicas $\omega_{i}^{o}$, y un factor de amortiguamiento suficientemente grande [esto es, $\xi_{\mathrm{i}} \geq 0.71$ como está reportado en la literatura del control lineal (Stephanopoulos, 1984)]. En caso contrario, si $n_{\mathrm{i}}$ es un número impar, entonces $\left(n_{\mathrm{i}}-1\right)$ polos son asignados de la misma forma, el $n_{\mathrm{i}}$-ésimo polo debe fijarse como un polo real con frecuencia caracteristica $\omega_{i}^{o}$. Recordando que los valores de referencia corresponden para un valor $s_{\mathrm{o}}=1$.

Resumiendo, los polos de la dinámica de referencia del error de salida $\left[v_{i}\right.$ en (3.47) o (3.75)] se asignan bajo el siguiente criterio (para $s_{0}=1$ ):

$$
\begin{aligned}
& \left.\gamma_{i}(1), \gamma_{i+1}(1)=-\omega_{i}^{o}\left(\xi_{i} \pm \sqrt{1-\xi_{i}^{2}} j\right), \quad i=1,3, \ldots, n_{i}-1\right\} \quad \text { si es } n_{i} \text { par } \\
& \left.\begin{array}{l}
\gamma_{i}(1), \gamma_{i+1}(1)=-\omega_{i}^{o}\left(\xi_{i} \pm \sqrt{1-\xi_{i}^{2}} j\right), \quad i=1,3, \ldots, n_{i}-2 \\
\gamma_{n_{i}}(1)=-\omega_{i}^{o}
\end{array}\right\} \quad \text { si es } n_{i} \text { impar }
\end{aligned}
$$

A partir de estos polos de referencia se determinan las ganancias de referencia $\left[k_{1}^{\mathrm{i}}(1), \ldots, k_{\mathrm{n}_{\mathrm{i}}}^{\mathrm{i}}(1)\right]$ por comparación de los coeficientes de las dos ecuaciones polinomiales dadas en (3.92). Las ganancias de referencias obtenidas mediante este procedimiento se listan en la Tabla 3.2 (el detalle del desarrollo para la obtención de estas ganancias se encuentra en el Apéndice F). La asignación de polos escalada en tiempo-s $s_{\mathrm{o}}\left[\gamma_{i}\left(s_{o}\right)=s_{o} \gamma_{i}(1), s_{o}>0\right]$ se muestra en la Figura 3.4. En contraste con la asignación de polos de Butterworth (ver Figura 3.3), aquí el diseñador puede evitar localizar los polos cerca del eje imaginario, lo cual es equivalente a prevenir la divergencia o la pobre robustez del estimador. 
Tabla 3.2 Ganancias de referencia para el estimador 225999

\begin{tabular}{|c|c|}
\hline & \\
\hline $1-P$ & $\omega_{i}^{o}$ \\
\hline $2-P / 1-P I$ & $2 \xi \omega_{i}^{o},\left(\omega_{i}^{o}\right)^{2}$ \\
\hline $3-P / 2-P I$ & $(2 \xi+1) \omega_{i}^{o},(2 \xi+1)\left(\omega_{i}^{o}\right)^{2},\left(\omega_{i}^{o}\right)^{3}$ \\
\hline $4-P / 3-P I$ & $4 \xi \omega_{i}^{o},\left(4 \xi^{2}+2\right)\left(\omega_{i}^{o}\right)^{2}, 4 \xi\left(\omega_{i}^{o}\right)^{3},\left(\omega_{i}^{o}\right)^{4}$ \\
\hline $5-P / 4-P I$ & $(4 \xi+1) \omega_{i}^{o},\left(4 \xi^{2}+4 \xi+2\right)\left(\omega_{i}^{o}\right)^{2},\left(4 \xi^{2}+4 \xi+2\right)\left(\omega_{i}^{o}\right)^{3},(4 \xi+1)\left(\omega_{i}^{o}\right)^{4},\left(\omega_{i}^{o}\right)^{5}$ \\
\hline
\end{tabular}

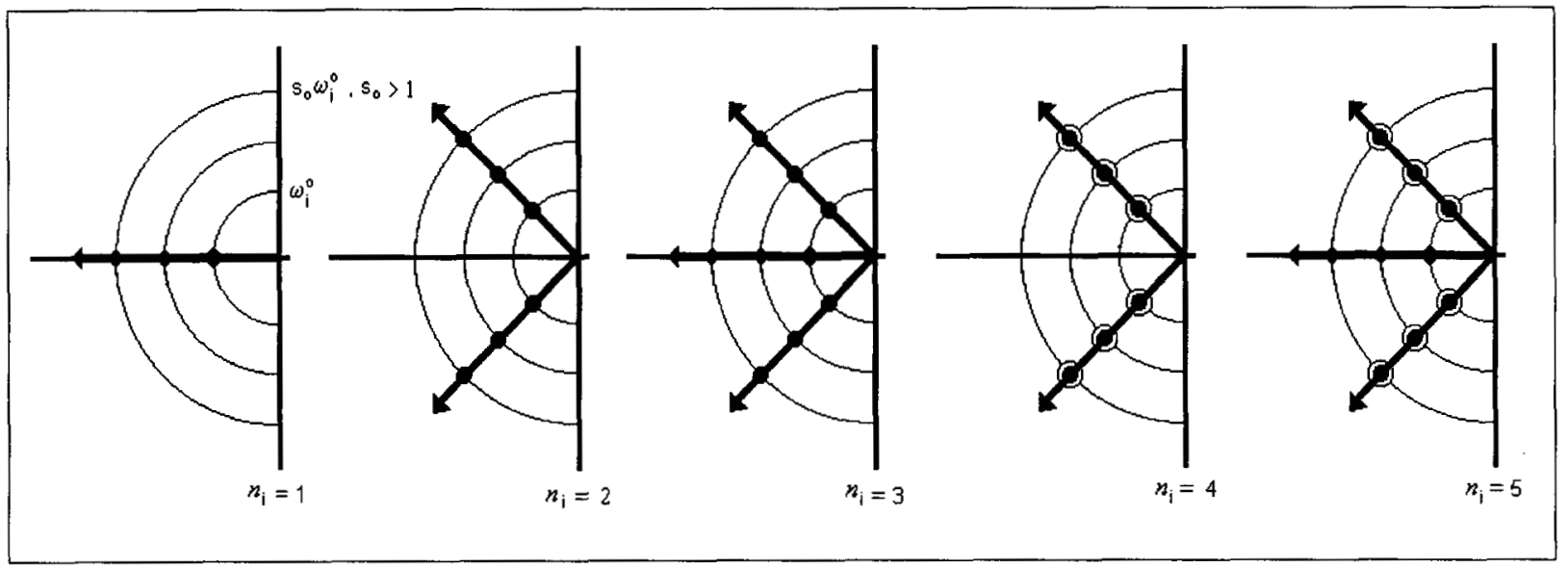

Figura 3.4 Localización de polos para el estimador de estados

\subsection{Consideraciones para la implementación de un estimador}

En ingenieria de procesos, un diseño de estimación geométrico no lineal debiera llevarse a cabo dentro de un marco metodológico, donde las etapas de la estrategia de estimación sean interpretadas y apoyadas con el conocimiento físico, modelado, métodos, y criterios de ingenieria asociados a la planta que en particular se considera. Por una parte, la teoria de estimación geométrico debe dar un marco conceptual no lineal, el soporte teórico, y un procedimiento sistemático del diseño de estimación; y por otra parte, la ingenieria de procesos debe determinar los objetivos y 
restricciones del diseño de estimación. De tal forma que la resolubilidad y las condiciones de convergencia se proporcionen con significado fisico, y se contribuya con una alta sistematización, identificando e interpretando las capacidades de estimación inherentes asi como las limitaciones asociadas a la física e ingeniería del sistema no lineal en particular. De aqui que la aplicabilidad y factibilidad de manipulación de un estimador geométrico no lineal reside en la unión de la metodologia de la teoria de estimación geométrico no lineal con las estrategias establecidas en ingenieria de proceso.

Pese a que el FEK (filtro extendido de Kalman) es la metodologia de estimación más ampliamente usada en los trabajos de investigación, éste no toma en cuenta las consideraciones anteriores para su implementación. Ya que no se explota la interpretación física del proceso en particular para la construcción - sintonización del mismo. Para finalizar con este capítulo sobre el marco teórico de estimación no lineal, en la Tabla 3.3 se muestran algunas caracteristicas importantes en un esquema de estimación, comparando el FEK con el estimador geométrico no lineal aqui propuesto; observándose que las ventajas del estimador geométrico no lineal son esencialmente: la sistematización de la construcción-sintonización y el criterio de convergencia. 
Tabla 3.3 Comparación del FEK y el estimador geométrico no lineal

\begin{tabular}{|c|c|c|}
\hline Caracteristica & FEK & Estimador No Lineal \\
\hline $\begin{array}{l}\text { Funciona en: } \\
\text { (a) simulación } \\
\text { (b) experimentación }\end{array}$ & $\begin{array}{l}\mathrm{Si} \\
\mathrm{Si}\end{array}$ & $\begin{array}{l}\mathrm{Si} \\
\mathrm{Si}\end{array}$ \\
\hline $\begin{array}{l}\text { Aplicable a plantas: } \\
\text { (a) Observables } \\
\text { (b) Detectables }\end{array}$ & $\begin{array}{l}\mathrm{Si} \\
\mathrm{No}\end{array}$ & $\begin{array}{l}\mathrm{Si} \\
\mathrm{Si}\end{array}$ \\
\hline Criterios de convergencia & No & $\mathrm{Si}$ \\
\hline Dimensión de la dinámica & $n(n+1) / 2$ & $n+p$ \\
\hline Matrices de ganancias & $Q_{n x n}, R_{m x m}$ & $K_{u}, K_{o}, K_{I}$ \\
\hline Número de ganancias $\left(\mathrm{n}_{\mathrm{g}}\right)$ & $\begin{array}{l}\text { teórica: } \\
n_{\mathrm{g}}=m(n-1) / 2+m(m-1) / 2 \\
\text { präctica: } \\
n_{\mathrm{g}}=n+m\end{array}$ & $\begin{array}{l}\text { Diseño proporcional: } \\
n_{\mathrm{g}}=v+\kappa \leq n \\
\text { Diseño prop. - integral: } \\
n_{\mathrm{g}}=v+\kappa+m \leq(n+m)\end{array}$ \\
\hline Sintonización & prueba y error & $\begin{array}{l}\text { Técnica sistemática } \\
\text { (asignación de polos) }\end{array}$ \\
\hline Manejo de entradas & si (ad hoc) & si, filtro y estimación \\
\hline $\begin{array}{l}\text { Sentido físico en la } \\
\text { selección de ganancias }\end{array}$ & No & $\begin{array}{l}\text { si, en térmicos de la física y } \\
\text { la dinámica natural del } \\
\text { proceso. }\end{array}$ \\
\hline
\end{tabular}

* $n=$ número de estados, $m=$ número de salidas, $p=$ número de entradas exógenas, $\kappa=$ índice de observabilidad global 


\section{Capítulo 4}

\section{ESTIMACIÓN DE ESTADOS EN}

\section{REACTORES DE COPOLIMERIZACIÓN}

En este capitulo se establece una metodologia para la selección de la estructura de estimabilidad. En principio existen 116 estructuras posibles para el reactor de copolimerización. Con algunas consideraciones de indole práctica pueden reducirse a 22 estructuras. Despuês, en la etapa de análisis, se plantean las condiciones de resolubilidad, y las medidas de estimabilidad para las estructuras definidas. Finalmente, en la etapa de diseño, se construyen, sintonizan, e implementan cuatro estimadores (con diferentes grados de estimabilidad) para compararlos entre sí, y corroborar las conjeturas realizadas en la etapa de análisis. 


\subsection{El problema de estim abilidad}

Como se estableció en el Capítulo 1, la inferencia de los estados y de las variables de calidad es un problema importante en la ingenieria de reactores de copolimerización con fuertes implicaciones en el monitoreo, el diseño, la operación, y el control del proceso. Por ello, el objetivo en este capitulo es proponer una metodología de estimación no lineal para inferir las variables clave (2.5) de: (a) calidad (composición instantánea, conversión, y peso molecular peso-promedio), (b) seguridad (fracción de sólidos), y (c) velocidad de producción en reactores de copolimerización en solución; mediante el empleo de mediciones secundarias (2.2) en linea de: densidad, indice de refracción, temperatura, y volumen; y conociendo las entradas exógenas o de control (2.6): flujos de alimentación y de descarga, temperaturas de alimentación (de monómeros y solvente) en el reactor, y concentraciones de alimentaciôn de reactivos.

El esquema de estimación se establece en dos etapas: la primera, etapa de análisis, consiste en la selección de (los grados de libertad): la estructura de estimabilidad y los parámetros de sintonización, tales que aseguren la convergencia-RE del estimador. Esto es, deben satisfacerse las condiciones de resolubilidad establecidas en el Lema 3.1, equivalente a la Definición 3.2. La segunda, etapa de diseño, consiste en la construcción (aplicando directamente el Teorema 3.1 o 3.2), sintonización e implementación del (los) estimador (es).

La metodologia propuesta muestra la factibilidad de:

(a) Diseñar un estimador con interpretación fisica, esto es, explotando el conocimiento del comportamiento estacionario y dinámico del reactor, las propiedades del copolimero, las dependencias de las variables medidas con las de interés, etc.

(b) Determinar el grado de estimabilidad, mediante la verificación de las condiciones de la Definición 3.2.

(c) Conocer a priori la convergencia, el desempeño y la robustez del estimador, a partir de la etapa de análisis; donde el Lema 3.1 se usa como una medida de la estimabilidad robusta exponencial.

Estos aspectos se abordan en cada una de las siguientes subsecciones: En la Sección 4.2 se establecen las posibles estructuras de estimabilidad para el reactor continuo de copolimerización en solución (caso general independiente del sistema de reacción en particular), y se seleccionan algunas de ellas bajo criterios y suposiciones basados en la física del problema. En la Sección 4.3 se evalúan las condiciones de resolubilidad (Definición 3.2 o Lema 3.1) para las estructuras de estimabilidad seleccionadas, se comparan entre sî, y se analiza a priori cual será el 
comportamiento dinámico del estimador (convergencia-desempeño-robustez) para el caso particular de la copolimerización de MMA-AV en solución. En la Sección 4.4 se construyen y sintonizan los estimadores P y PI para cada una de las estructuras de estimabilidad seleccionadas. Y finalmente, en la Sección 4.5 se presenta la implementación de los estimadores (mediante simulaciones numéricas), corroborando las conjeturas dichas en la Sección 4.3 sobre el comportamiento dinámico de los estimadores.

\subsection{Estructuras de estim abilidad}

Para determinar las estructuras de estimabilidad $S=\left(k, x_{I}\right)$ posibles, tomemos en cuenta que el reactor (2.6) tiene nueve estados $(x)$ y cuatro salidas $(y)$, además que el mapa $h$ y los primeros siete mapas $\left(f_{1}, \ldots, f_{7}\right)$ no dependen de los momentos de la DLC $\left(x_{8}=\mu_{0}, x_{9}=\mu_{2}\right)$, lo cual implica que los momentos no son estados del mapa observable $x_{1}(3.14)$, y que la dimensión de $x_{1}$ puede variar entre 4 y 7 :

$$
\left\{x_{8}, x_{9}\right\} \notin x_{1}, \quad \therefore 4 \leq \kappa_{1}+\kappa_{2}+\kappa_{3}+\kappa_{4} \leq 7
$$

Las posibles combinaciones en el conjunto $k=\left(\kappa_{1}, \kappa_{2}, \kappa_{3}, \kappa_{4}\right)$ que cumplan la condición (4.1) son: $(1,1,1,1),(2,1,1,1),(1,2,1,1),(1,1,2,1),(1,1,1,2),(2,2,1,1)$, $(2,1,2,1), \quad(2,1,1,2), \quad(1,2,2,1), \quad(1,1,2,2), \quad(1,2,1,2), \quad(3,1,1,1), \quad(1,3,1,1), \quad(1,1,3,1)$, $(1,1,1,3), \quad(2,2,2,1), \quad(2,2,1,2), \quad(2,1,2,2), \quad(1,2,2,2), \quad(3,2,1,1), \quad(3,1,2,1), \quad(3,1,1,2)$, $(2,3,1,1), \quad(1,3,2,1), \quad(1,3,1,2), \quad(2,1,3,1), \quad(1,2,3,1), \quad(1,1,3,2), \quad(2,1,1,3), \quad(1,2,1,3)$, $(1,1,2,3), \quad(4,1,1,1), \quad(1,4,1,1), \quad(1,1,4,1), y(1,1,1,4)$.

Asimismo, para cada conjunto $k$ se puede seleccionar varios conjuntos de $x_{\mathrm{I}}$. Matemáticamente existe un conjunto $\Sigma^{\star}$ de 116 elementos de estructuras de estimabilidad (ver Tabla 4.1), las cuales pueden se clasificadas en cuatro subconjuntos de acuerdo a la dimensión $\kappa$ del vector $x_{I}$ (card $\Sigma$ es la cardinalidad o número de elementos en $\Sigma$ ):

$$
\begin{array}{ll}
\Sigma^{*}=\left\{\Sigma_{1}^{*}, \Sigma_{2}^{*}, \Sigma_{3}^{*}, \Sigma_{4}^{*}\right\}, & \operatorname{card}\left(\Sigma^{*}\right)=116 \\
\Sigma_{i}^{*}=\left\{S=\left(k, x_{I}\right) \mid k \in K_{i}, x_{I} \in X_{i}^{I}\right\}, & \operatorname{dim} x_{I}\left(\text { en } \Sigma_{i}^{*}\right)=\kappa=3+i, \\
& \operatorname{card}\left(\Sigma_{1}^{*}, \Sigma_{2}^{*}, \Sigma_{3}^{*}, \Sigma_{4}^{*}\right)=(6,40,50,20)
\end{array}
$$

donde el vector de observabilidad $\left(K_{i}\right)$ y la coordenada $x_{I}\left(X_{\mathrm{i}}^{\top}\right)$ están dados en la Tabla 4.1 . 
Tabla 4.1 Estructuras de estimabilidad posibles (matemáticamente) para un reactor de copolimerización.

\begin{tabular}{|c|c|c|c|c|c|}
\hline$\Sigma_{i}^{*}$ & $\kappa$ & $k=\left(x_{1}, k_{2}, k_{3}, x_{j}\right)$ & $X_{i}$ & $x_{T}^{I}$ & $\operatorname{Card} \Sigma_{t}^{*}$ \\
\hline \multirow{6}{*}{$\Sigma_{1}^{*}$} & \multirow{6}{*}{4} & \multirow{6}{*}{$(1,1,1,1)$} & $\left(m_{1}, m_{2}, T, V\right)$ & $\left(p_{1}, p_{2}, i, \mu_{0}, \mu_{2}\right)$ & \multirow{6}{*}{6} \\
\hline & & & $\left(m_{1}, p_{1}, T, V\right)$ & $\left(m_{2}, p_{2}, i, \mu_{0}, \mu_{2}\right)$ & \\
\hline & & & $\left(m_{l}, p_{2}, T, V\right)$ & $\left(m_{2^{\prime}}, p_{p}, i, \mu_{0}, \mu_{2}\right)$ & \\
\hline & & & $\left(m_{2}, p_{1}, T, V\right)$ & $\left(m_{p}, p_{2}, i, \mu_{0}, \mu_{2}\right)$ & \\
\hline & & & $\left(m_{2}, p_{2}, T, V\right)$ & $\left(m_{p}, p_{p}, i, \mu_{0}, \mu_{2}\right)$ & \\
\hline & & & $\left(p_{1}, p_{2}, T, V\right)$ & $\left(m_{1}, m_{2}, i, \mu_{0}, \mu_{2}\right)$ & \\
\hline \multirow{10}{*}{$\Sigma_{2}^{*}$} & \multirow{10}{*}{5} & \multirow{10}{*}{$\begin{array}{l}(2,1,1,1) \\
(1,2,1,1) \\
(1,1,2,1) \\
(1,1,1,2)\end{array}$} & $\left(m_{1}, m_{2}, p_{l}, T, V\right)$ & $\left(p_{2}, i, \mu_{0}, \mu_{2}\right)$ & \multirow{10}{*}{40} \\
\hline & & & $\left(m_{1}, m_{2^{\prime}} p_{2}, T, V\right)$ & $\left(p_{p}, i, \mu_{0}, \mu_{2}\right)$ & \\
\hline & & & $\left(m_{1}, p_{1}, p_{2}, T, V\right)$ & $\left(m_{2}, i, \mu_{0}, \mu_{2}\right)$ & \\
\hline & & & $\left(m_{2}, p_{1}, p_{2}, T, V\right)$ & $\left(m_{1}, i, \mu_{0}, \mu_{2}\right)$ & \\
\hline & & & $\left(m_{l}, m_{2}, i, T, V\right)$ & $\left(p_{1}, p_{2}, \mu_{0}, \mu_{2}\right)$ & \\
\hline & & & $\left(m_{l}, p_{l}, i, T, V\right)$ & $\left(m_{2}, p_{2}, \mu_{0}, \mu_{2}\right)$ & \\
\hline & & & $\left(m_{1}, p_{2}, i, T, V\right)$ & $\left(m_{2}, p_{p}, \mu_{0}, \mu_{2}\right)$ & \\
\hline & & & $\left(m_{2}, p_{l}, i, T, V\right)$ & $\left(m_{1}, p_{2}, \mu_{0}, \mu_{2}\right)$ & \\
\hline & & & $\left(m_{2}, p_{2^{\prime}} i, T, V\right)$ & $\left(m_{1}, p_{1}, \mu_{0}, \mu_{2}\right)$ & \\
\hline & & & $\left(p_{1}, p_{2}, i, T, V\right)$ & $\left(m_{1}, m_{2}, \mu_{0}, \mu_{2}\right)$ & \\
\hline \multirow{5}{*}{$\Sigma_{3}^{*}$} & \multirow{5}{*}{6} & \multirow{5}{*}{$\begin{array}{l}(2,2,1,1),(2,1,2,1) \\
(2,1,1,2),(1,2,2,1) \\
(1,1,2,2),(1,2,1,2) \\
(3,1,1,1),(1,3,1,1) \\
(1,1,3,1),(1,1,1,3)\end{array}$} & $\left(m_{l}, m_{2}, p_{1}, p_{2}, T, V\right)$ & $\left(i, \mu_{0}, \mu_{2}\right)$ & \multirow{5}{*}{50} \\
\hline & & & $\left(m_{1}, m_{2}, p_{l}, i, T, V\right)$ & $\left(p_{2}, \mu_{0}, \mu_{2}\right)$ & \\
\hline & & & $\left(m_{1}, m_{2^{2}}, p_{2}, i, T, V\right)$ & $\left(p_{1}, \mu_{0}, \mu_{2}\right)$ & \\
\hline & & & $\left(m_{l}, p_{p}, p_{z}, i, T, V\right)$ & $\left(m_{2}, \mu_{0}, \mu_{2}\right)$ & \\
\hline & & & $\left(m_{2}, p_{1}, p_{2}, i, T, V\right)$ & $\left(m_{l}, \mu_{2}\right)$ & \\
\hline \multirow{10}{*}{$\Sigma_{4}^{*}$} & \multirow{10}{*}{7} & $(2,2,2,1),(2,2,1,2)$ & \multirow{10}{*}{$\left(m_{1}, m_{2}, p_{l}, p_{2}, i, T, V\right)$} & \multirow{10}{*}{$\left(\mu_{0}, \mu_{2}\right)$} & \multirow{10}{*}{20} \\
\hline & & $(2,1,2,2),(1,2,2,2)$ & & & \\
\hline & & $(3,2,1,1),(3,1,2,1)$ & & & \\
\hline & & $(3,1,1,2),(2,3,1,1)$ & & & \\
\hline & & $(1,3,2,1),(1,3,1,2)$ & & & \\
\hline & & $(2,1,3,1),(1,2,3,1)$ & & & \\
\hline & & $(1,1,3,2),(2,1,1,3)$ & & & \\
\hline & & $(1,2,1,3),(1,1,2,3)$ & & & \\
\hline & & $(4,1,1,1),(1,4,1,1)$ & & & \\
\hline & & $(1,1,4,1),(1,1,1,4)$ & & & \\
\hline
\end{tabular}


Siguiendo el marco metodológico de las secciones 3.7 - 3.9 sobre la selección de una estructura de estimabilidad y aspectos para la implementación, tomemos en cuenta las siguientes consideraciones cinéticas y de ingenieria para delimitar un conjunto más pequeño de estructuras de estimabilidad:

(1) En un reactor industrial, los cambios en el volumen causados por aumento de la conversion - densidad (implicando una contracción de volumen) no pueden ser detectados por un indicador de nivel estándar o un sensor gravimétrico, lo cual significa que el indice de observabilidad del volumen debe ser uno:

$$
\kappa_{4}=1
$$

(2) Para eliminar la posibilidad de propagación excesiva de errores de modelado, se pedirá que el mapa $\varphi$ (3.12) incluya a lo sumo primeras derivadas de las velocidades de propagación y segundas derivadas de los mapas de densidad e indice de refracción, en el entendido que esta suposición se corroborará a posteriori (en la fase de implementación):

$$
\kappa_{1}, \kappa_{2}, \kappa_{3} \leq 2
$$

(3) Puesto que las primeras derivadas de densidad, indice de refracción, y temperatura tienen una fuerte dependencia con respecto a los estados $\left(m_{1}, m_{2}, p_{1}\right.$, $p_{2}$ ) comparando con el estado (i), entonces se seleccionará preferiblemente estos cuatro estados dentro del mapa observable $x_{\mathrm{I}}$.

A partir de estas consideraciones, el conjunto $\Sigma^{*}$ de 116 elementos se reduce a un conjunto $\Sigma$ de 22 elementos de estructuras de estimabilidad (ver Tabla 4.2):

$$
\begin{array}{ll}
\Sigma=\left\{\Sigma_{1}, \Sigma_{2}, \Sigma_{3}, \Sigma_{4}\right\}, & \operatorname{card}(\Sigma)=22 \\
\Sigma_{I}=\left\{S=\left(k, x_{I}\right) \mid k \in K_{i}, x_{I} \in x_{l i}\right\}, & \operatorname{dim} x_{l i}=\kappa=3+i, \\
& \operatorname{card}\left(\Sigma_{1}, \Sigma_{2}, \Sigma_{3}, \Sigma_{4}\right)=(6,12,3,1)
\end{array}
$$

Esta caracterización estructural se cumple para la clase completa de reactores de copolimerización en solución, dadas las mediciones de densidad, indice de refracción, temperatura y volumen; y es independientemente del sistema de reacción en particular y de la trayectoria a seguir. 
Tabla 4.2 Estructuras de estimabilidad factibles para implementación, para un reactor de copolimerización.

\begin{tabular}{|c|c|c|c|c|c|c|}
\hline$\Sigma_{\mathrm{i}}$ & $x$ & $\begin{array}{c}k= \\
\left(\kappa_{1}, \kappa_{2} \kappa_{p} \kappa_{p}\right)\end{array}$ & $\phi_{1}\left(x, x_{1}, b\right)$ & $X_{\mathbf{f}}$ & $x_{i}$ & $\begin{array}{c}\text { Card } \\
\Sigma_{i}\end{array}$ \\
\hline \multirow{6}{*}{$\Sigma_{1}$} & \multirow{6}{*}{4} & \multirow{6}{*}{$(1,1,1,1)$} & \multirow{6}{*}[\rho,\eta,T,V]{$_{\left(m_{1}, m_{2}, p_{1}, p_{2}, \mathrm{~T}, \mathrm{~V}\right)}^{\prime}$} & $\left(m_{1}, m_{2}, T, V\right)$ & $\left(p_{1}, p_{2}, i, \mu_{0}, \mu_{2}\right)$ & \multirow{6}{*}{6} \\
\hline & & & & $\left(m_{1}, p_{j}, T, V\right)$ & $\left(m_{2}, p_{2}, i, \mu_{0}, \mu_{2}\right)$ & \\
\hline & & & & $\left(m_{1}, p_{2}, T, V\right)$ & $\left(m_{2}, p_{1}, i, \mu_{0}, \mu_{2}\right)$ & \\
\hline & & & & $\left(m_{2}, p_{2}, T, V\right)$ & $\left(m_{1}, p_{2}, i, \mu_{0}, \mu_{2}\right)$ & \\
\hline & & & & $\left(m_{2}, p_{2}, T, V\right)$ & $\left(m_{p}, p_{p}, i, \mu_{0}, \mu_{2}\right)$ & \\
\hline & & & & $\left(p_{i}, p_{2}, T, V\right)$ & $\left(m_{1}, m_{2}, i, \mu_{0}, \mu_{2}\right)$ & \\
\hline \multirow{4}{*}{$\Sigma_{2}$} & \multirow{4}{*}{5} & \multirow{2}{*}{$(2,1,1,1)$} & \multirow{2}{*}[\rho,\dot{\rho},\eta,T,V]{$_{\left(m_{1}, m_{2}, p_{1}, p_{2}, i, \mathrm{~T}, \mathrm{~V}, u, b\right)}^{\prime}$} & $\left(m_{1}, m_{2}, p, T, V\right)$ & $\left(p_{2}, i, \mu_{0}, \mu_{2}\right)$ & \multirow{4}{*}{12} \\
\hline & & & & $\left(m_{1}, m_{2}, p_{2}, T, V\right)$ & $\left(p_{1}, i, \mu_{0}, \mu_{2}\right)$ & \\
\hline & & $(1,1,2,1)$ & {$[\rho, \eta, \dot{\eta}, T, V]_{\left(m_{1}, m_{2}, p_{1}, p_{2}, i, \mathrm{~T}, \mathrm{~V}, u, b\right)}^{\prime}$} & $\left(m_{p}, p_{p}, p_{2}, T, V\right)$ & $\left(m_{2}, i, \mu_{0}, \mu_{2}\right)$ & \\
\hline & & $(1,1,1,2)$ & {$\left[\rho, \eta, T, \dot{T}, V_{\left(m_{1}, m_{2}, p_{1}, p_{2}, i, \mathrm{~T}, \mathrm{~V}, u, b\right)}^{\prime}\right.$} & $\left(m_{2^{\prime}}, p_{1}, p_{2}, T, V\right)$ & $\left(m_{p}, i, \mu_{0}, \mu_{2}\right)$ & \\
\hline \multirow{3}{*}{$\Sigma_{3}$} & \multirow{3}{*}{6} & $(2,2,1,1)$ & {$[\rho, \dot{\rho}, \eta, \dot{\eta}, T, V]_{\left(m_{1}, m_{2}, p_{1}, p_{2}, i, \mathrm{~T}, \mathrm{~V}, u, b\right)}^{r}$} & \multirow{3}{*}{$\left(m_{1}, m_{2}, p_{1}, p_{2}, T, V\right)$} & \multirow{3}{*}{$\left(i, \mu_{0}, \mu_{2}\right)$} & \multirow{3}{*}{3} \\
\hline & & $(2,1,2,1)$ & {$[\rho, \dot{\rho}, \eta, T, \dot{T}, V]_{\left(m_{1}, m_{2}, p_{1}, p_{2}, i, \mathrm{~T}, \mathrm{~V}, u, b\right)}$} & & & \\
\hline & & $(1,2,2,1)$ & {$[\rho, \eta, \dot{\eta}, T, \dot{T}, V]_{\left(m_{1}, m_{2}, p_{1}, p_{2}, i, \mathrm{~T}, \mathrm{v}, u, b\right)}^{\prime}$} & & & \\
\hline$\Sigma_{4}$ & 7 & $(2,2,2,1)$ & {$[\rho, \dot{\rho}, \eta, \dot{\eta}, T, \dot{T}, V]_{\left(m_{1}, m_{2}, p_{1}, p_{2}, i, \mathrm{~T}, \mathrm{v}, u, b\right)}^{\prime}$} & $\left(m_{p}, m_{2}, p_{p}, p_{2}, i, T, V\right)$ & $\left(\mu_{0}, \mu_{2}\right)$ & 1 \\
\hline
\end{tabular}

\subsection{Condiciones de resolubilidad}

La evaluación de la propiedad de estimabilidad-RE equivale a determinar/analizar las medidas del Lema 3.1, para las 22 estructuras de estimabilidad del conjunto $\Sigma$ (Tabla 4.2). De las caracteristicas físicas del reactor de copolimerización (i.e., los acotamiento de los estados y entradas del reactor son: $0 \leq c_{\mathrm{i}} \leq 1, c_{\mathrm{i}}=\left\{m_{1}, m_{2}, p_{1}, p_{2}, i\right.$, $\left.m_{1 e}, m_{2 e}, p_{1 e}, p_{2 e}, i_{e}\right\} ; 0 \leq T_{i} \leq T_{\max }, T_{\mathrm{i}}=\left\{T, T_{1 e}, T_{2 e}, T_{s e}, T\right\} ; 0 \leq q_{\mathrm{i}} \leq T_{\max }, q_{\mathrm{i}}=\left\{q, q_{1}, q_{2}, q_{\mathrm{s}}\right\} \mathrm{y}$ $0 \leq \mathrm{V} \leq 2 \mathrm{~L}$ ) se sabe que los mapas $\phi_{I}(3.11), \varphi(3.12)$, y $f_{I I}(3.16)$ tienen funciones $(\mathcal{U}$, $b)-,(x, \mathcal{U}, v, b)-, \mathrm{y}(\mathcal{U}, y, b)$ - sensibilidad acotadas, respectivamente. Esto es, los jacobianos de los mapas $\rho, \dot{\rho}, \ddot{\rho}, \eta, \dot{\eta}, \ddot{\eta}, T, \dot{T}, \ddot{T}, \mathrm{y} V$ con respecto a $(x, \mathcal{u}, y, v, b)$ son finitos [la función sensibilidad, $s_{\beta}^{\alpha}$, está definida en (3.84) - (3.85)]: 


$$
\begin{aligned}
& s_{u}^{\phi_{l}}(t)<s_{\phi_{l}, u}^{*}, \quad s_{b}^{\phi_{l}}(t)<s_{\phi_{1}, b}^{*} ; \\
& s_{x}^{\varphi}(t)<s_{\varphi, x}^{*}, \quad s_{u}^{\varphi}(t)<s_{\varphi, u}^{*}, \quad s_{\nu}^{\varphi}(t)<s_{\varphi, \nu}^{*}, \quad s_{b}^{\varphi}(t)<s_{\varphi, b}^{*} ; \\
& s_{u}^{f_{I I}}(t)<s_{f_{I}, u}^{*}, \quad s_{y}^{f_{I I}}(t)<s_{f_{I,}, y}^{*}, \quad s_{b}^{f_{I I}}(t)<s_{f_{I I}, b}^{*}
\end{aligned}
$$

Por tanto, siempre se cumplen que las funciones de sensibilidad del Lema 3.1 están acotadas. De aquí que el análisis de la estimabilidad-RE equivale a evaluar las medidas restantes del Lema 3.1, que corresponden a determinar (a lo largo de la trayectoria de operación):

(a) el $x_{l}$-condicionamiento del mapa $\phi_{I}$, equivalente a verificar que

(b) el $x_{I I}$-condicionamiento del mapa $f_{I I}$, equivalente a verificar que

(c) el margen de estabilidad $l_{\mathrm{s}}$ del mapa $f_{I I}$, equivalente a verificar que

Las condiciones (a) y (b) garantizan la estimabilidad robusta (i.e. cuando existen errores en los parámetros del modelo) para que $\phi_{I}$ sea $\mathrm{Rx}$, -invertible y que la dinámica no observable sea RE-estable, y son equivalentes a verificar que:

$$
\begin{gathered}
c_{x_{l}}^{\phi_{I}}(t)<c_{\phi_{l}, x_{l}}^{*} \\
c_{x_{I I}}^{f_{I I}}(t)<c_{f_{I I}, x_{l l}}^{*}
\end{gathered}
$$

donde $c_{\phi_{t}, x_{l}}^{*}$ y $c_{f_{l}, x_{l l}}^{*}$ son constantes finitas.

Sin embargo para verificar la estimabilidad nominal (i.e. cuando el modelo es perfecto) sólo se requiere que:

$$
\begin{array}{ccc}
\operatorname{det}\left(\phi_{I x_{l}}\right) \neq 0 \quad \text { o } & \operatorname{Rango}\left(\phi_{I x_{I}}\right)=\kappa ; \quad \phi_{I x_{I}}=\partial \phi_{I} / \partial x_{I} \\
l_{s}(t)>0 &
\end{array}
$$

donde la primer medida garantiza que $\phi_{l}$ sea $x_{l}$-invertible, y la segunda que la dinámica no observable sea únicamente E-estable.

Como punto de comparación y para destacar la importancia entre la resolubilidad nominal y la resolubilidad robusta, se evaluaron tanto las medidas nominales (4.9)(4.10) como las medidas de robustez (4.7)-(4.8) para cada una de las estructuras del conjunto $\Sigma$ (4.5), a lo largo de la trayectoria de operación establecida en la Sección 2.2.2 para el reactor de copolimerización de MMA-AV en solución. De este análisis se obtuvieron los siguientes resultados comparativos: 
(1) Por una parte, las medidas nominales se cumplen para todas las estructuras de estimabilidad, lo cual indica que sin errores en los parámetros del modelo, cualquiera de las estructuras de estimabilidad del conjunto $\mathrm{S}$ son factibles de emplear para diseñar e implementar el estimador. Y puesto que la velocidad de convergencia más rápida se obtiene con $\kappa$ grandes (ver Sección 3.7), entonces uno seleccionaria cualquiera de las estructuras del subconjunto $\Sigma_{4}$. No obstante, este resultado no es el criterio adecuado para seleccionar una estructura, ya que en un caso práctico no se cuenta con un modelo perfecto del proceso.

(2) Al evaluar las medidas robustas, el $x_{l}$-condicionamiento del mapa observable de todas las estructuras del conjuntos $\Sigma$ fueron aceptables (con $c_{x_{t}}^{\phi_{t}}(t)<10^{10}$ ); $\sin$ embargo, la medida del $x_{I I}$-condicionamiento de la dinámica no observable resulto ser excesivamente mal condicionado (con $c_{x_{I I}}^{f_{I}}(t)>10^{10}$ ) para las siguientes cinco estructuras:

$$
\begin{aligned}
& \left\{(1,1,1,1),\left(m_{1}, p_{2}, T, V\right)\right\} \in \Sigma_{1}, \\
& \left\{(1,1,1,1),\left(p_{1}, p_{2}, T, V\right)\right\} \in \Sigma_{1}, \\
& \left\{(2,1,1,1),\left(m_{1}, m_{2}, p_{1}, T, V\right)\right\} \in \Sigma_{2}, \\
& \left\{(1,2,1,1),\left(m_{1}, m_{2}, p_{1}, T, V\right)\right\} \in \Sigma_{2} \\
& \left\{(1,1,2,1),\left(m_{1}, m_{2}, p_{1}, T, V\right)\right\} \in \Sigma_{2}
\end{aligned}
$$

las cuales se descartaron para diseño e implementación de un estimador robusto (aunque nominalmente si tienen soluciōn). Reduciéndose el conjunto $\Sigma$ a 17 estructuras factibles para implementación.

Para discernir entre las 17 estructuras de estimabilidad restante, simplemente se compararon entre sí las medidas de robustez de cada una de éstas. De las cuales, las cuatro estructuras $\left(S_{1}, S_{2}, S_{3}, S_{4}\right)$ con el mejor balance de robustez en cada subconjunto $\left(\Sigma_{1}, \Sigma_{2}, \Sigma_{3}, \Sigma_{4}\right)(4.5)$ son las siguientes:

$$
\begin{aligned}
& S_{1}=\left\{(1,1,1,1),\left(m_{1}, m_{2}, T, V\right)\right\} \in \Sigma_{1}, \\
& S_{2}=\left\{(1,1,1,1),\left(m_{1}, m_{2}, p_{2}, T, V\right)\right\} \in \Sigma_{2}, \\
& S_{3}=\left\{(2,1,1,1),\left(m_{1}, m_{2}, p_{1}, p_{2}, T, V\right)\right\} \in \Sigma_{3}, \\
& S_{4}=\left\{(1,2,1,1),\left(m_{1}, m_{2}, p_{1}, p_{2}, i, T, V\right)\right\} \in \Sigma_{4}
\end{aligned}
$$


En las Figura 4.1 y Figura 4.2 se muestran las medidas de estimabilidad nominal y robusta, respectivamente, para las estructuras $\left(S_{1}, S_{2}, S_{3}, S_{4}\right)$, observándose que:

(i) La medida nominal $\operatorname{det}\left(\phi_{I x_{I}}\right)$ siempre es diferente de cero, indicando existencia de invertibilidad para $x_{I}$.

(ii) El margen de estabilidad o velocidad de convergencia se incrementa cuando la dimensión del mapa no observable $(n-\kappa)$ disminuye, lo cual significa que la tendencia a inestabilidad es menor con dimensiones pequeñas del mapa no observable $x_{I I}$.

(iii) Con la estructura $S_{1}$ (con la dinámica observable más pequeña) se obtiene la mejor robustez de la dinámica del error observable (i.e., el $x_{I}$-condicionamiento más pequeño), pero también se obtiene la peor robustez de la dinámica no observable (i.e., el $x_{I I}$-condicionamiento más grande).

(iv) La estructura $S_{4}$ (con la dinámica observable más grande) se comporta en el lado opuesto a la estructura $S_{1}$, se obtiene la mejor robustez de la dinámica no observable, pero la peor robustez de la dinámica observable.

(v) En todos los casos, los periodos dinámicamente excitados hacia ignición (ver lineas discontinuas en las figuras 2.3 y 2.4) en la trayectoria de operación favorecen la robustez de la dinámica observable, pero deterioran la robustez de la dinámica no observable.

Estos resultados sugieren que $S_{1}$ y $S_{4}$ son los casos extremos para velocidad de convergencia y robustez, por lo que $S_{2}$ o $S_{3}$ son las estructuras de estimabilidad candidatas para obtener el mejor funcionamiento robusto del estimador, o equivalente a, obtener el mejor compromiso entre la velocidad de reconstrucción de estados y la tolerancia a errores de modelado.

Fisicamente hablando, estos resultados indican que en nuestra trayectoria de operación, para el reactor de copolimerización de MMA-AV, el orden de preferencia para colocar los estados en la partición $x_{\mathrm{I}}$ es la siguiente: primero los monómeros libres $\left(m_{1}, m_{2}\right)$, luego la conversión del monómero menos reactivo $\left(p_{2}\right)$, luego la conversión del monómero más reactivo $\left(p_{l}\right)$, y finalmente el iniciador $(i)$. 


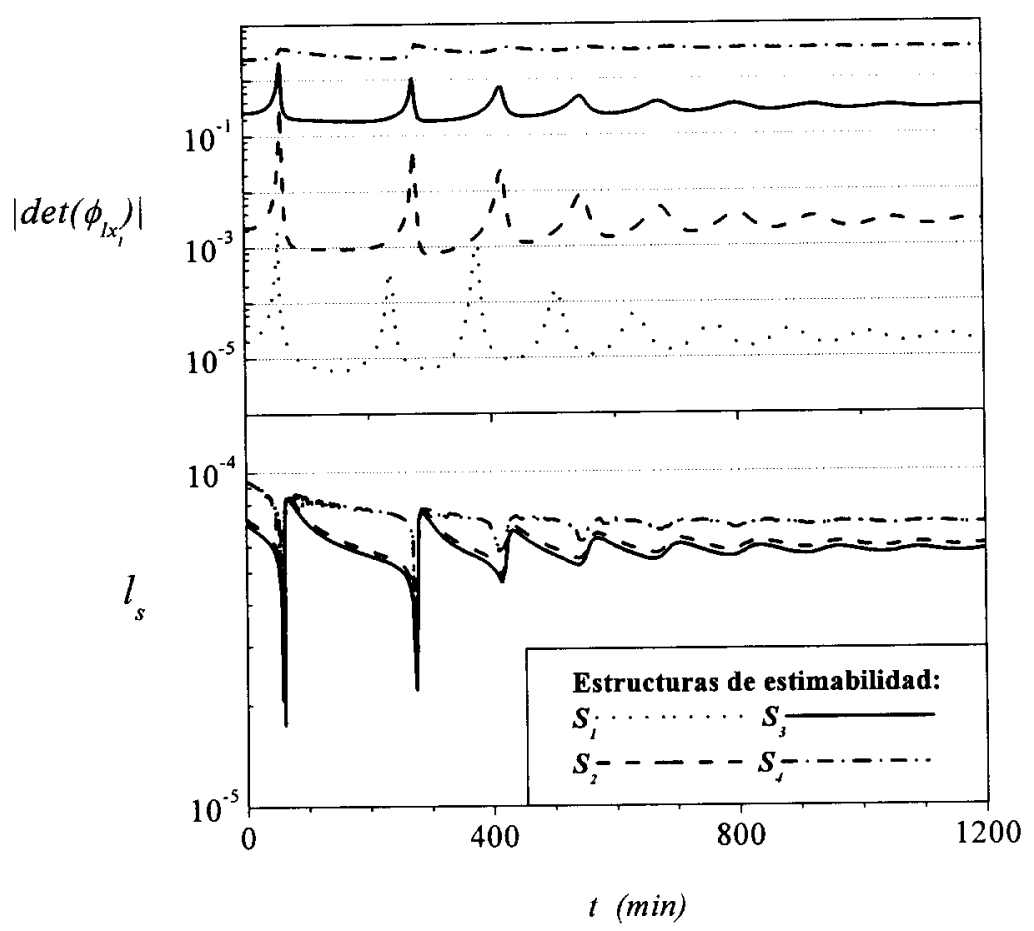

Figura 4.1 Funciones de medida de la propiedad de estimabilidad-N (nominal).

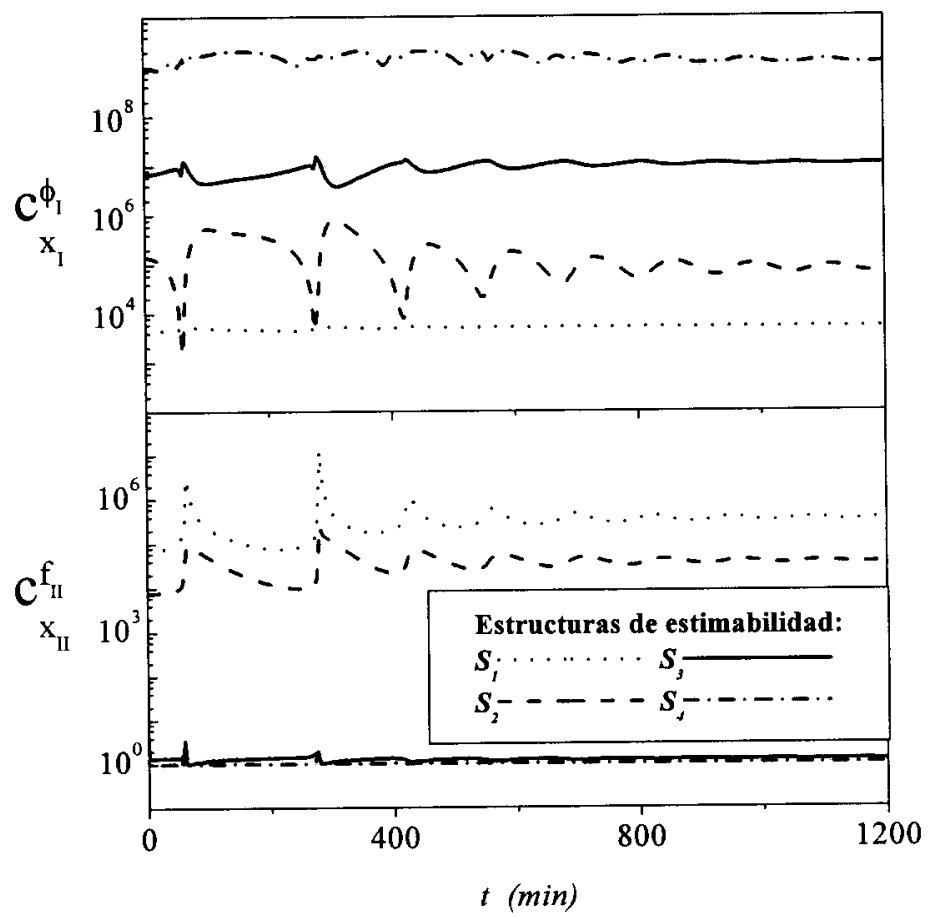

Figura 4.2 Funciones de medida de la propiedad de estimabilidad-RE (robusta exponencial) 


\subsection{Construcción y Sint onización de los estimadores}

Para una estructura de estimabilidad $S$ dada, la aplicación directa del Teorema 2 (Capitulo 3) proporciona la construcción del estimador-P no lineal, para condiciones nominales (con $\beta=b$ y $\hat{x}_{o} \neq x_{o}$ ); y la aplicación del Teorema 3 (Capitulo 3) proporciona la construcción del estimador-PI no lineal, para condiciones robustas (con $\beta \neq b$ y $\left.\hat{x}_{o} \neq x_{o}\right)$. Donde el mapa $\phi_{l}\left(x, x_{u}, b\right)$ está definido en la Tabla 4.2, y el exoobservador se construyo únicamente para las entradas $u^{\prime}(t)$ conocidas y variantes en tiempo (definidas en la Sección 2.2.2 para la trayectoria de operación diseñada):

$$
u^{\prime}(t)=\left[m_{l e}, T_{l e}, T_{2 e}, T_{s e}\right]^{\prime}, \quad u^{\prime}(t) \subset u(t)
$$

y cuyos indices exo-observables, para las cuatro estructuras $\left(S_{l}, S_{2}, S_{3}, S_{4}\right)$,son:

$$
\left(v_{1}, v_{2}, v_{3}, v_{4}\right)=(1,1,1,1)
$$

Con respecto al esquema de sintonización, éste se estableció en la Sección 3.8. Las ganancias de referencia para el exo-observador, $K_{\mathrm{u}}(1)$, están tabuladas en la Tabla 3.2; y las ganancias de referencia para el estimador-P, $K_{\mathrm{o}}(1)$, o del estimador-PI, $\left[K_{0}(1), K_{\mathrm{I}}(1)\right]$, se encuentran en la Tabla 3.3. De acuerdo a estas tablas, las ganancias de referencia estản en función de frecuencias y factores de amortiguamiento de referencia. De acuerdo al estudio realizado en la Sección 3.8 y Apéndice $F$, los factores de amortiguamiento se seleccionaron, para las cuatro estructuras $\left(S_{1}, S_{2}, S_{3}\right.$, $S_{4}$ ), como:

$$
\xi_{i}=0.71
$$

Por otra parte, las frecuencias de referencia se escogieron con base en el estudio dinámico del reactor (hecho en el Capitulo 2): el reactor tiene un tiempo de asentamiento de aproximadamente $\tau_{a}=800 \mathrm{~min}$ (ver figura 2.4), que equivale a un tiempo característico de $\tau_{c}=200 \min \left(\tau_{a} \approx 4 \tau_{c}\right)$, o bien su frecuencia natural característica es $\omega_{r}=1 / 200 \mathrm{~min}^{-1}\left(\omega_{r}=1 / \tau_{c}\right)$. A partir de esta última frecuencia se seleccionaron las frecuencias de referencia del exo-observador como:

$$
\begin{array}{ll}
\omega_{l}^{u}=8 \omega_{r}=1 / 25 \mathrm{~min}^{-1} & \left(\text { param }_{l e}\right) \\
\omega_{i}^{u}=(20 / 3) \omega_{r}=1 / 30 \mathrm{~min}^{-1}, \quad i=2,3,4 & \left(\text { para }_{l e}, T_{2 e}, y T_{s e}\right)
\end{array}
$$

y del estimador como:

$$
\begin{array}{ll}
\omega_{1}^{o}=\omega_{2}^{o}=\omega_{r} / 2=1 / 400 \mathrm{~min}^{-1} & (\text { para } \rho y \eta) \\
\omega_{3}^{o}=\omega_{4}^{o}=2 \omega_{r}=1 / 100 \mathrm{~min}^{-1} & (\text { para } \mathrm{y} \mathrm{V})
\end{array}
$$

Aquí se definió $\omega_{3}^{o}, \omega_{4}^{o}>\omega_{1}^{o}, \omega_{2}^{o}$ porque las mediciones de temperatura y de volumen son mucho más rápidas en respuesta dinámica que densidad e indice de refracción, y además $T$ y $V$ son estados directos del sistema; y $\omega_{i}^{u}>\omega_{i}^{o}$ ya que en este caso el exoobservador se emplea únicamente para filtrar ruido, por lo que su velocidad de 
convergencia puede hacerse mucho más rápida que la del estimador. Y por último, los parámetros de aceleración/retardo $\left(s_{\mathrm{o}}, s_{\mathrm{u}}\right)$ se escogieron como

$$
\left(s_{u}, s_{o}\right)=(1,10)
$$

La forma de selección de los parámetros de ajuste (4.15)-(4.18) significa que la dinámica del exo-observador es más rápida que la del error de salida de estimación, y ésta a su vez es diez veces más rápida que la dinámica natural del estimador. Es importante señalar que las ganancias resultantes se emplearon para todos los estimadores (esto es, sin importar el grado de observabilidad $k$ ) sin reajuste de parámetros.

\subsection{Implementación de los estimadores}

A continuación se presentan los resultados comparativos del funcionamiento de los estimadores $\left(E_{1}, E_{2}, E_{3}, E_{4}\right)$ asociados a las estructuras de estimabilidad $\left(S_{1}, S_{2}, S_{3}, S_{4}\right)$. Se consideran tres situaciones: (1) el funcionamiento nominal, con errores en los estados iniciales; (2) el funcionamiento robusto, con errores en los estados iniciales y en los parámetros del modelo; y (3) el funcionamiento robusto, con errores en los estados iniciales, en los parámetros del modelo, y en las mediciones.

Por funcionamiento nominal del estimador entendemos el comportamiento cuando existen errores en los estados iniciales pero no hay errores de modelado, por lo cual seleccionamos el diseño-P; y este estudio debe verse como un medio para entender y comparar el caso práctico de interés: el funcionamiento robusto implementando el diseño-PI para obtener mayor tolerancia a errores iniciales, de modelado, y en la mediciones.

\subsubsection{Funcionamiento Nominal}

En la Figura 4.3 se presenta y se compara la salida $z$ estimada, generada por el funcionamiento- $\mathrm{N}$ (nominal) de los estimadores-P. Los estimadores fueron implementados con los estados iniciales listados en la Tabla 2.4. Los resultados son los siguientes:

(1) Como se esperaba, en todos los casos, las salidas estimadas (lineas delgadas en la Figura 4.3) convergen asintóticamente (i.e., sin sesgo) a sus valores reales (lineas gruesas en la Figura 4.3). 


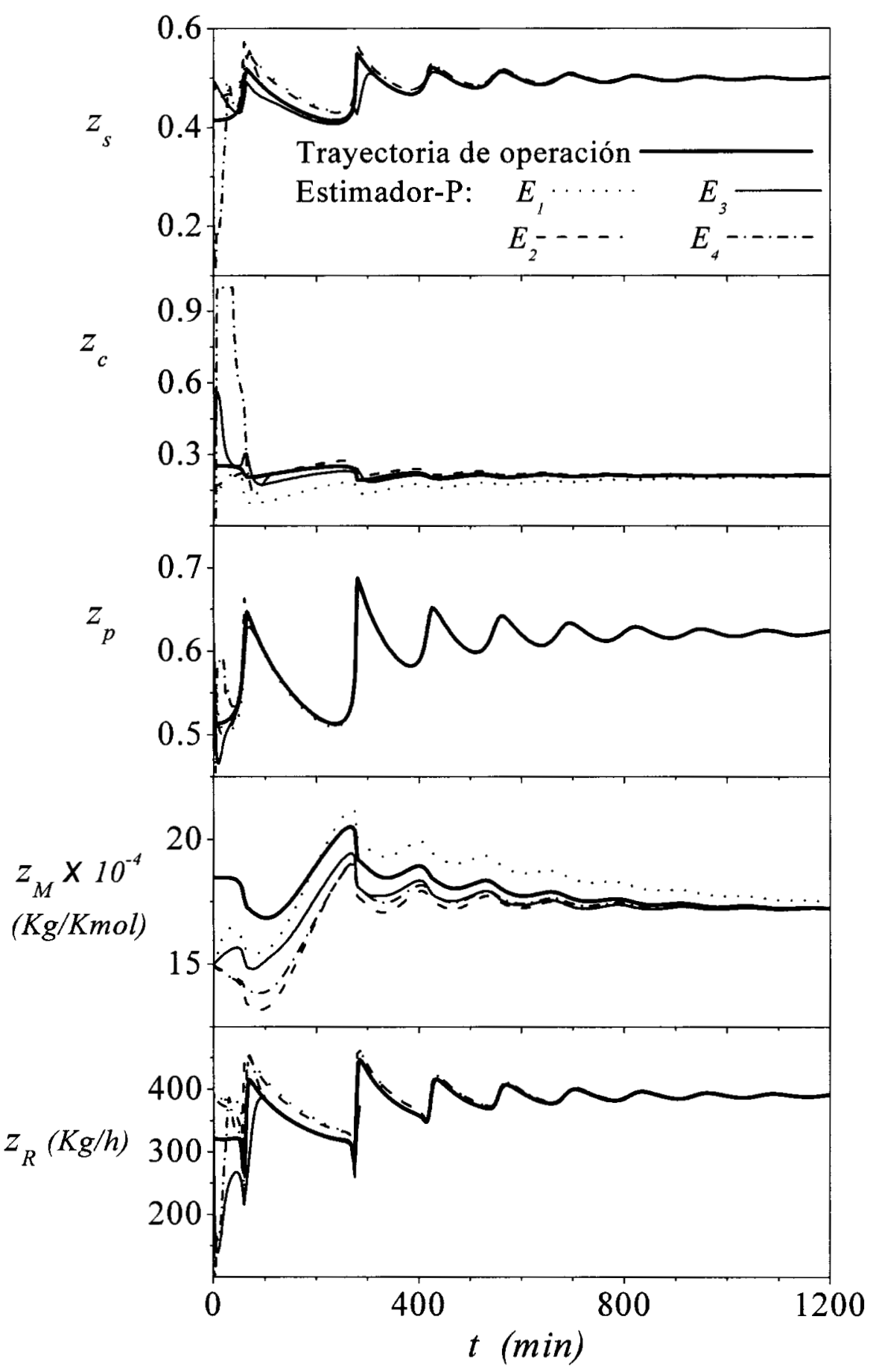

Figura 4.3 Estimados de las salidas de interés a de los estimadores $E_{1}, E_{2}, E_{3}$, y $E_{4}$, con errores en las condiciones iniciales. 
(2) Con el estimador $E_{4}$ (con la dimensión observable más grande) se obtiene la velocidad de convergencia más rápida y con el disparo en el error inicial más grande. De manera opuesta, con el estimador $E_{1}$ (con la dimensión observable más pequeña) se obtiene la velocidad de convergencia más lenta y con el disparo en el error inicial más pequeño.

(3) Comparando entre las salidas $z$ estimadas, la fracción de sólidos $\left(z_{s}\right)$ y la conversión $\left(z_{p}\right)$ son las salidas con la convergencia más rápida porque éstas están más ligadas a la dinámica observable (i.e., de $x_{l}$ ), mientras que el peso molecular $\left(z_{M}\right)$ es la salida con la velocidad de convergencia más lenta, debido a que depende significativamente de la dinámica no observable (i.e., de $x_{\text {II }}$ ).

(4) Excepto por el disparo inicial en el estimado de la composición instantánea $\left(z_{c}\right)$, $\underline{e l}$ estimador $E_{4}$ genera el mejor funcionamiento- $N$ global. Los estimadores $E_{2}$ y $E_{3}$ constituyen los casos intermedios de desempeño, mientras que $E_{1}$ y $E_{4}$ son los casos extremos.

(5) Conforme los errores en los estados iniciales disminuyen, el estimador $E_{4}$ definitivamente proporciona el mejor funcionamiento- $\mathrm{N}$, y con el estimador $E_{1}$ se obtiene el "peor" funcionamiento-N.

Cabe señalar que, las características del funcionamiento- $N$ aqui resultantes corroboran las conjeturas para la propiedad de estimabilidad nominal realizadas en la sección 4.3 .

\subsubsection{Funcionamiento Robusto}

En la Figura 4.4 se presenta el funcionamiento-R (robusto) de cada uno de los estimadores-PI. Los estimadores fueron implementados nuevamente con los estados iniciales listados en la Tabla 2.4, con los errores en los parámetros del modelo listados en la Tabla 2.5; los resultados son los siguientes:

(1) En todos los casos, hay convergencia con sesgos asintóticos que son más pequeños para las salidas más ligadas a la dinámica observable, lo cual concuerda con la caracterización establecida en el Corolario 3.2.

(2) Comparando con el funcionamiento-N de la Figura 4.3, el estimador $E_{4}$ (el cual tenía el mejor desempeño nominal) experimenta la peor degradación y genera el peor funcionamiento-R: ahora existen disparos periódicos decadentes que se asientan en aproximadamente 1500 min. Esta característica es más pronunciada en las salidas $z_{s}, z_{c}, z_{p}$, y $z_{R}$ que son las más directamente ligadas a la dinámica observable, y es menos pronunciada en la salida $z_{M}$ que está ligada a la dinámica no observable. 
(3) Por otra parte, el estimador $E_{1}$ (el cual tenía el "peor" desempeño nominal comparativo) experimenta la menor degradación, con un desempeño semejante al funcionamiento-N.

(4) El funcionamiento de los estimadores $E_{1}$ y $E_{4}$ corroboran nuevamente las caracteristicas predichas por la teoría de estimación geométrico no lineal: que las estructuras con grados de observabilidad $\kappa$ grandes (i.e. $E_{4}$ ) permiten reconstruir los estados más rápidamente, peros son muy sensibles a errores de modelado; y de lo contrario, las estructuras con $\kappa$ pequeños (i.e. $E_{l}$ ) permiten mayor tolerancia a errores de modelado, pero reconstruyen los estados más lentamente.

(5) Con el estimador $E_{3}$ se obtiene el mejor funcionamiento- $R$, en términos de un balance adecuado entre la velocidad de reconstrucción de estados y la tolerancia a errores de modelado. El estimador $E_{2}$ tiene una respuesta similar al $E_{3}$, excepto en la estimación de la salida $z_{M}$ que tiene un tiempo más largo de convergencia. La diferencia entre $E_{2}$ y $E_{3}$ es únicamente la reconstrucción de un estado más en $E_{3}$ (la del estado $p_{1}$ ).

(6) En el estimador $E_{3}$ (con el mejor funcionamiento-R), los sesgos asintóticos de las salidas $z$ son como sigue: muy pequeños (i.e. casi imperceptibles) para $z_{s}, z_{c}$, y $z_{p}$, y pequeños (i.e. aceptables) para $z_{M}$, y $z_{R}$. Estos sesgos pueden reducirse aún más mediante la calibración o reajuste de un conjunto adecuado de parámetros del modelo empleando algunas mediciones ocasionales de la calidad del producto $\left(z_{s}\right.$, $z_{c}, z_{p}, z_{M}$ ) en tiempo real (López et al., 2000; López-Serrano et al., 1999a, 1999b).

Recapitulando, al probar el funcionamiento de los 22 estimadores candidato (con las estructuras de estimabilidad de la Tabla 4.2), se encontró que el estimador $E_{3}$ produce el mejor funcionamiento-R, en segundo lugar el estimador $E_{2}$, y cinco estimadores [con las estructuras de estimabilidad dadas en (4.11)] divergieron por un excesivo mal $x_{I I}$-condicionamiento de la dinámica no observable. Estos resultados contundentes están en total acuerdo con las conjeturas sugeridas a partir de la caracterización cuantitativa de la propiedad de estimabilidad-RE (Sección 4.3). Además, valida la suposición hecha de obtener un conjunto pequeño $\Sigma, \Sigma(4.5) \subset \Sigma^{*}$ (4.2), de estructuras de estimabilidad candidatas, a partir de considerar principalmente que $\kappa_{4}=1$ (4.3) y que $\kappa_{1}, \kappa_{2}, \kappa_{3} \leq 2$ (4.4); para prevenir una propagación excesiva de los errores de modelado debido a la presencia de derivadas parciales de alto orden en las matrices de ganancias, $G$ y $G_{I}$, del estimador. 


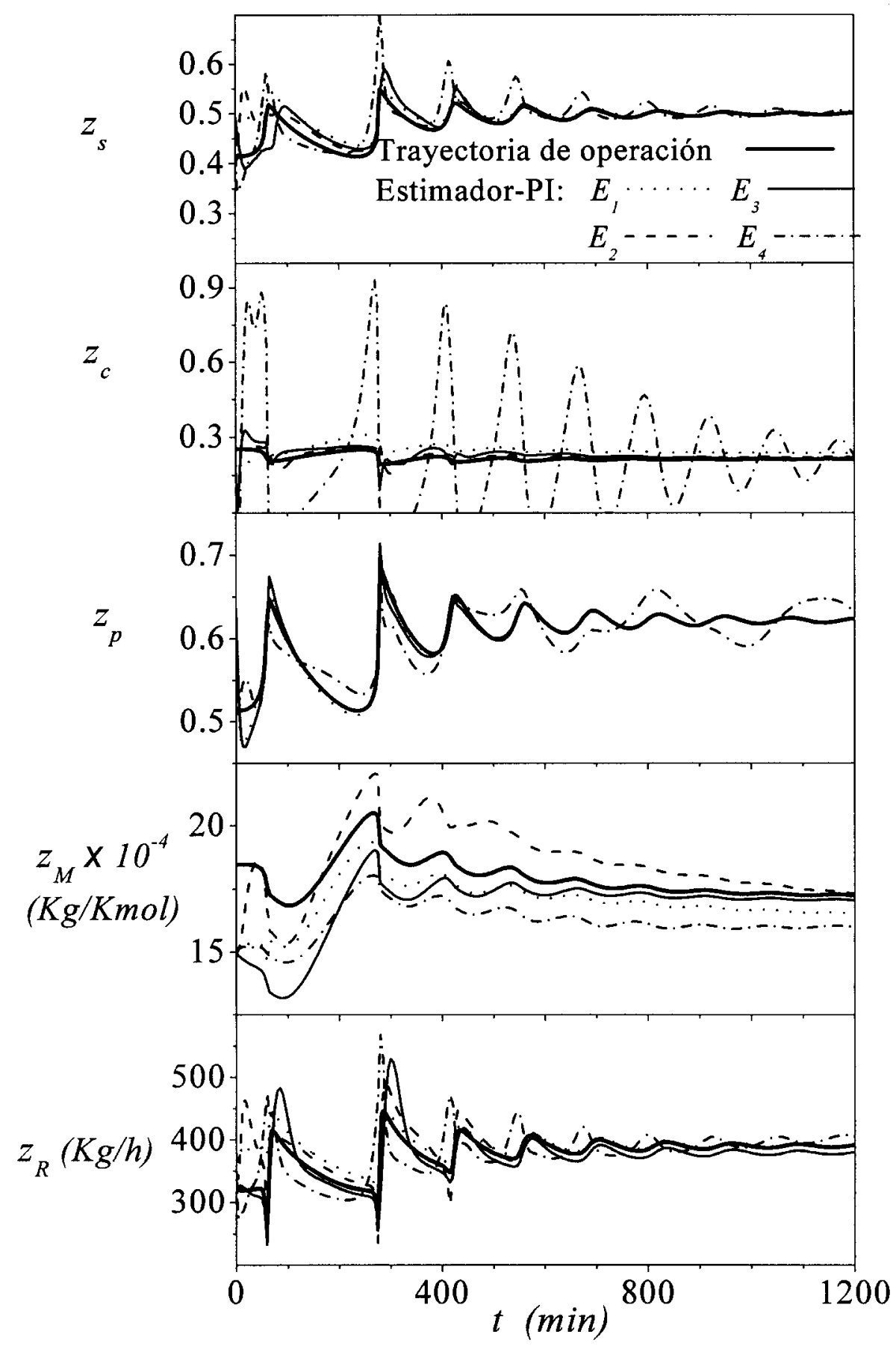

Figura 4.4 Estimados de las salidas de interés a de los estimadores $E_{1}, E_{2}, E_{3}$, y $E_{4}$, con errores en las condiciones iniciales y en los parámetros del modelo. 


\subsubsection{Tolerancia de ruido en las mediciones}

La prueba restante para determinar la robustez del diseño de estimación no lineal propuesto, consiste en verificar la tolerancia del estimador ante posible ruido en la adquisición de las mediciones, tanto en las salidas $y(t)$ como en las entradas exógenas $u^{\prime}(t)$. Para esta última prueba se seleccionó el estimador $E_{3}$, ya que indudablemente el estimador $E_{4}$ no tolera esta prueba debido a su alta sensibilidad a errores, mientras a que los estimadores $E_{1}$ y $E_{2}$ tendrán un desempeño por abajo del estimador $E_{3}$ (de acuerdo a las conclusiones de la sección anterior).

El ruido en las mediciones se simuló numéricamente, de tal forma que se reproduce en buena medida al común de los instrumentos de medición en linea (en este caso: densímetro, refractómetro, termopar o RTD, y sensor de nivel). El ruido fue inyectado en intervalos de tiempo de $10 \mathrm{~s}$, usando variables aleatorias Gaussianas, con desviaciones de (ver Figura 4.5a): $1^{\circ} \mathrm{K}$ para las temperaturas $T, T_{l e}, T_{2 e}, \mathrm{y} T_{s e}, 0.001$ para la concentración de alimentación $m_{l e}, 1 \mathrm{Kg} / \mathrm{m}^{3}$ para la densidad $\rho, 0.001$ para el indice de refracción $\eta$, y de $0.01 \mathrm{~m} 3$ para el volumen $V$. El estimador-PI se implementó con los estados iniciales y errores en los parámetros listados en las Tablas 2.4 y 2.5 respectivamente.

En la Figura 4.5 se muestran los estados $x$, las salidas medidas $y$, las salidas de interés $z$, y las entradas exógenas $u^{\prime}$, para la trayectoria de operación y (Con propósitos de comparación) para los funcionamientos nominal (sin ruido en mediciones) y robusto (con ruido en mediciones). Los resultados son los siguientes:

(1) El funcionamiento-R del estimador-PI con ruido presenta estimados promedio que se parecen a los obtenidos para el caso sin ruido.

(2) Como se esperaba, el estimador- $\mathrm{R}$ (comparado con el estimador- $\mathrm{N}$ ) tiene una velocidad de convergencia más lenta, más oscilatoria, y con un sesgo pequeño pero aceptable.

(3) La propagación de ruido es más pronunciada en las salidas $z$ que dependen más fuertemente de la dinámica observable.

(4) En nuestra trayectoria de operación en particular, la propagación de ruido es aceptable. Pero si la propagación de éste fuese un problema, se recomienda emplear pre-filtros rápidos para las mediciones ruidosas antes de ser alimentadas al estimador no lineal. 


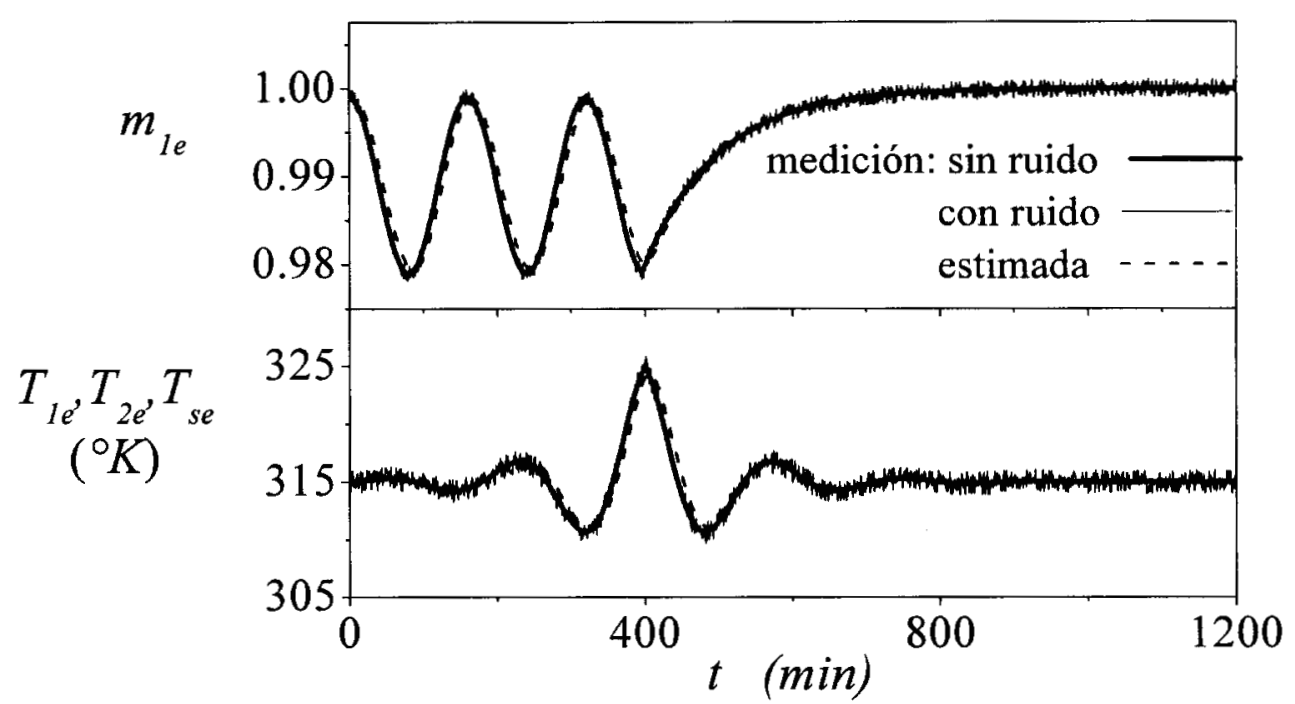

Figura 4.5 a Evolución del estimador con $E_{3}$ : Entradas exógenas $u^{\prime}(t)$.

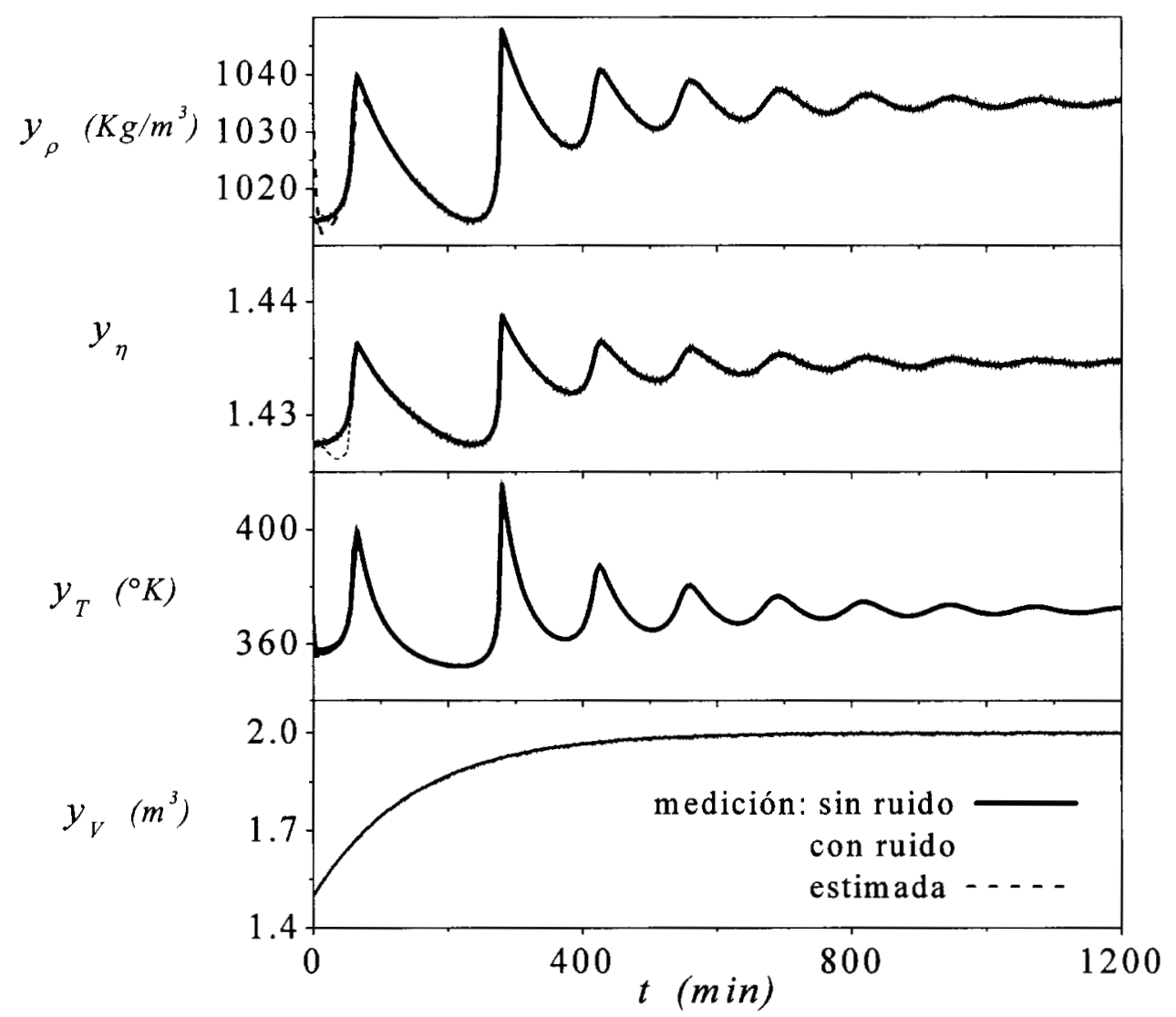

Figura $4.5 \mathrm{~b}$ Evolución del estimador con $E_{3}$ : Salidas medidas $y(t)$. 


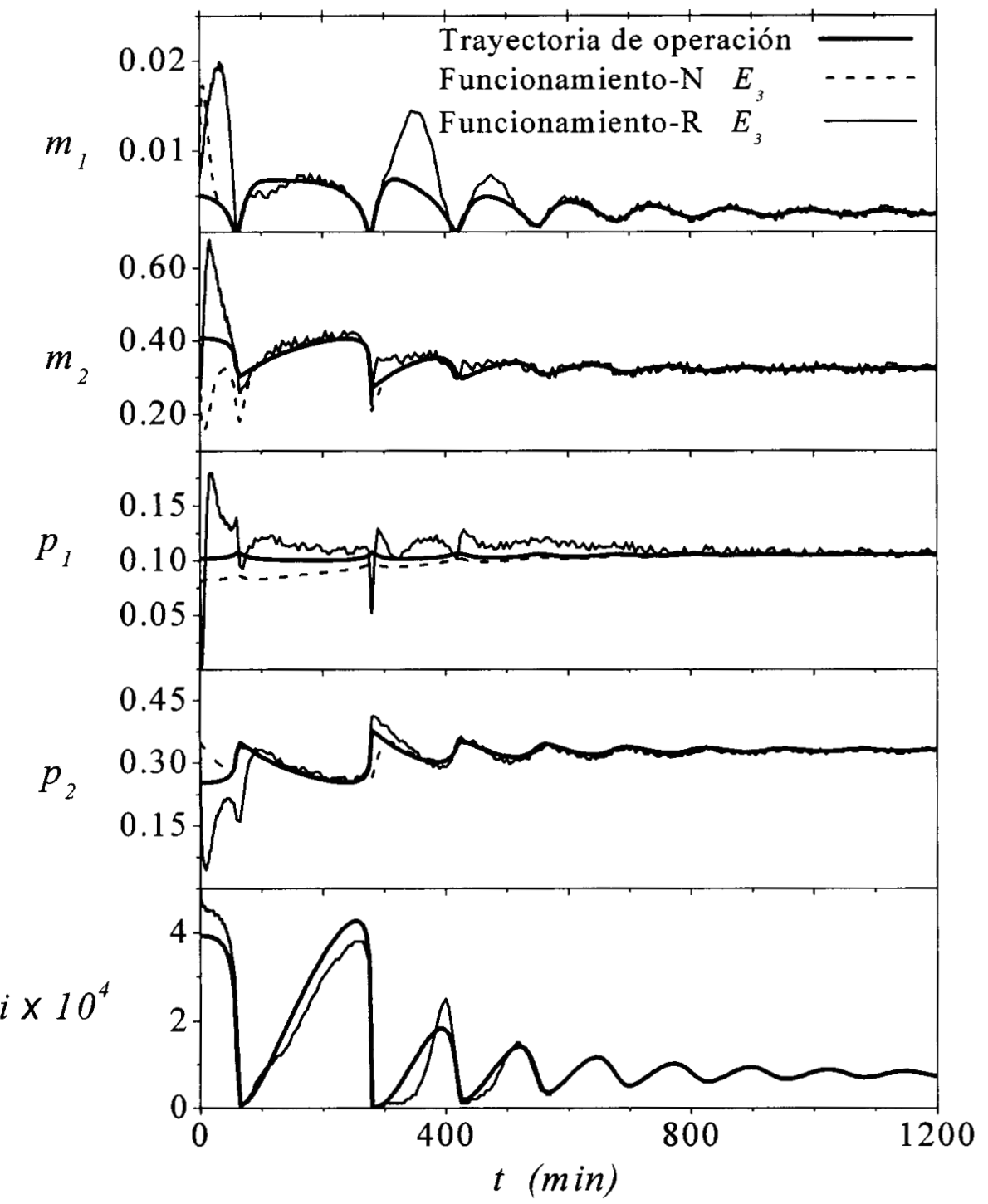

Figura 4.5 c Evolución del estimador con $E_{3}$ : Estados del reactor $x(t)$. 


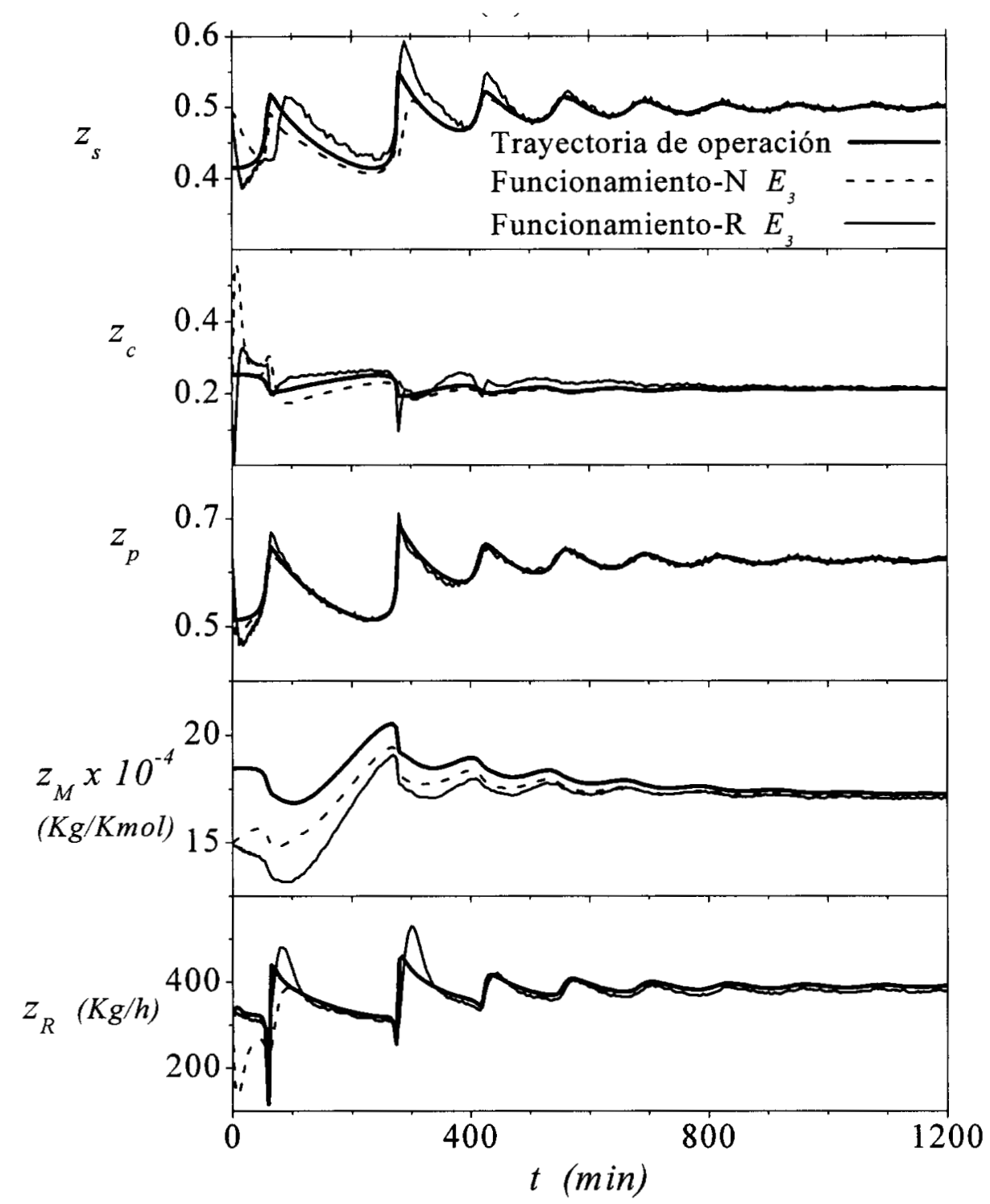

Figura $4.5 \mathrm{~d}$ Evolución del estimador con $E_{3}$ : Salidas de interés $z(t)$. 


\subsection{Resumen}

Hasta aqui se ha presentado una metodologia de estimación geométrico no lineal con (a) un criterio de convergencia robusto, (b) un procedimiento de construcción sistemático, (c) una técnica de sintonización simple, sistemática, y con interpretación física, y (d) la estructura de estimabilidad como un grado de libertad importante en el diseño. La estructura de estimabilidad fue conformada por el vector de observabilidad y por la coordenada de estados observables, y su selección fue crucial para el funcionamiento robusto en el que se tiene que mantener un balance entre desempeño y robustez. La propiedad de estimabilidad fue reformulada con medidas que evalúan y comparan: la robustez de la dinâmica observable, la robustez de la dinámica no observable, la sensibilidad de la interconexión de las dinámicas observable y no observable, y la velocidad de reconstrucción de los estados.

El diseño de estimación no lineal propuesto en el Capitulo 3 y ejemplificado con el problema de estimación en reactores de polimerización, mostró la factibilidad de inferir las medidas de seguridad, calidad, y velocidad de producción mediante mediciones secundarias en linea de temperaturas, flujos, volumen, densidad e indice de refracción. Tomando en cuenta consideraciones prácticas como son posibles errores en parámetros del modelo y en la adquisición de las mediciones (ruido).

Por otra parte, se mostró que la mezcla de estrategias de la teoria de estimación no lineal con estrategias de la ingenieria de reactores de polimerización permite que la resolución del problema sea manejable y estructural. Esto, acoplado con procedimiento de construcción-sintonización altamente sistemático, permitió establecer como el funcionamiento robusto del estimador del reactor depende de la estructura de estimabilidad, y determinar que estructura de estimabilidad produciria el mejor funcionamiento robusto.

La metodologia propuesta es aplicable a problemas de estimación en reactores (continuos, semicontinuos, o por lotes) de polimerización en general (masa, solución, suspensión, emulsión, etc.). Recientemente se ha aplicado para abordar problemas de estimación de estados en: (i) homopolimerización en masa via radicales libres (Alvarez y López, 1999; Alvarez, 2000; Alvarez et al., 1999, 2000), y (ii) copolimerización en solución via radicales libres, empleando mediciones de presión de vapor, densidad, temperatura, y volumen (López y Alvarez, 1996, 1997). Asimismo la metodologia es extendible para: (i) la estimación de parámetros del modelo, como se ha hecho en microemulsión (López-Serrano et. al., 1999a, b), y en polimerización por etapas (López, 2000), y (ii) el diseño de estimadores con mediciones discretas, como se ha hecho en homopolimerización en solución (Hernández y Alvarez, 2000) y en polimerización por etapas (López et al., 2000). 


\title{
Capítulo 5
}

\section{CONTROL NO LINEAL CON}

\author{
RETROALIMENTACIÓN DE
}

\section{MEDICIONES}

En este último capitulo se presenta el esquema de control para el reactor continuo de copolimerización. Primero se construye el control no lineal exacto con retroalimentación de estados (i.e. bajo la suposición de que todos los estados son conocidos), definiendo las condiciones de resolubilidad, el criterio de convergencia , y la técnica de sintonización. Enseguida se presenta el esquema de control no lineal con retroalimentación de mediciones, el cual se construye mediante la combinación del control no lineal exacto con el estimador de estados a lazo abierto (definido en los capitulos anteriores). Y finalmente se implementan los controladores para la regulación del estado estacionario inestable del reactor, mostrando convergencia robustez ante errores en la condiciones iniciales, cambios en las entradas exógenas, y errores en los parámetros del modelo. 


\subsection{El problema de cont rol}

Consideremos la planta no lineal no autónoma MEMS (múltiples entradas múltiples salidas)

$$
\dot{x}=f[x, u(t), d(t), b], \quad y=h(x, b), \quad z=h_{z}(x, b) ; \quad x\left(t_{o}\right)=x_{o}
$$

con $n$ estados $(x), m$ salidas medibles $(y), m_{\mathrm{e}}$ salidas de interés $(z), m$ entradas de control $(u), p$ entradas exógenas $(d)$, y $n_{\mathrm{p}}$ parámetros del modelo $(b)$. Las entradas $u(t)$ y $d(t)$ son funciones del tiempo continuas por tramos; y los mapas $f, h$ y $h_{z}$ son suficientemente suaves (diferenciables) alrededor de la evolución $\mathrm{E}(t)$

$$
E(t)=\{x(t), u(t), d(t), y(t)\}
$$

donde la trayectoria (posiblemente inestable)

$$
x(t)=\theta_{x}\left[t, t_{o}, x_{o}, u(t), d(t), b\right]
$$

está determinada únicamente por el conjunto de datos $\left\{x_{o}, u(t), \mathrm{b}\right\}$. La planta debe ser operada alrededor de un estado estacionario nominal $\bar{x}$ o de una trayectoria nominal predeterminada $\bar{x}(t)$ (posiblemente inestables), con entradas nominales $[\bar{u}(t), \bar{d}(t)]$ y salida nominal $\bar{y}(t)$; esto es

$$
\begin{gathered}
\bar{E}(t)=\{\bar{x}(t), \bar{u}(t), \bar{d}(t), \bar{y}(t)\} \\
\bar{x}(t)=\theta_{x}\left[t, t_{o}, x_{o}, \bar{u}(t), \bar{d}(t), b\right], \quad \bar{y}=h(\bar{x}, b)
\end{gathered}
$$

El problema de control consiste en diseñar un controlador dinámico no lineal (con retroalimentación de mediciones)

$$
\begin{array}{lc}
\dot{\hat{x}}_{e}=f_{e}\left\{\hat{x}_{e}, \mu\left[\hat{x}_{e}, K_{c}(y-\bar{y})\right], d_{m}(t), \beta\right\}, & \hat{x}_{e o}=\hat{x}_{e}\left(t_{o}\right) \\
u=\mu\left[\hat{x}_{e}, K_{c}(y-\bar{y})\right], & \hat{x}_{e}=\left(\hat{x}_{d}{ }^{\prime}, \hat{x}_{m}{ }^{\prime}, \hat{x}^{\prime}\right)^{\prime}, \quad \operatorname{dim}\left(\hat{x}_{e}\right)=(v, m, n)
\end{array}
$$

que, construido con un vector de parámetros aproximado $\beta$, forzado por las mediciones $d_{\mathrm{m}}(t)$ y $y(t)$, y por un control con retroalimentación de estados $\mu\left[\hat{x}_{e}, K_{c}(y-\bar{y})\right]$, genera una trayectoria de la planta a lazo cerrado

$$
\begin{aligned}
& \dot{x}=f\left\{x, \mu\left[\hat{x}_{e}, K_{c}(y-\bar{y})\right], d(t), b\right\}, \quad y=h(x, b), \quad z=h_{z}(x, b) \\
& \dot{\hat{x}}_{e}=f_{e}\left\{\hat{x}_{e}, \mu\left[\hat{x}_{e}, K_{c}(y-\bar{y})\right], d_{m}(t), \beta\right\}, \quad \hat{y}=h(\hat{x}, b), \quad \hat{z}=h_{z}(\hat{x}, b)
\end{aligned}
$$

donde: (i) la trayectoria generada $x(t)$ converge-RE a la trayectoria nominal $\bar{x}(t)$ (5.5), (ii) el error de estimación de salida $(\hat{y}-y)$ y el error de regulación/seguimiento $(y-\bar{y})$ converge-RE con dinámicas LNPA (lineal, no-interactiva, de polo asignable). 


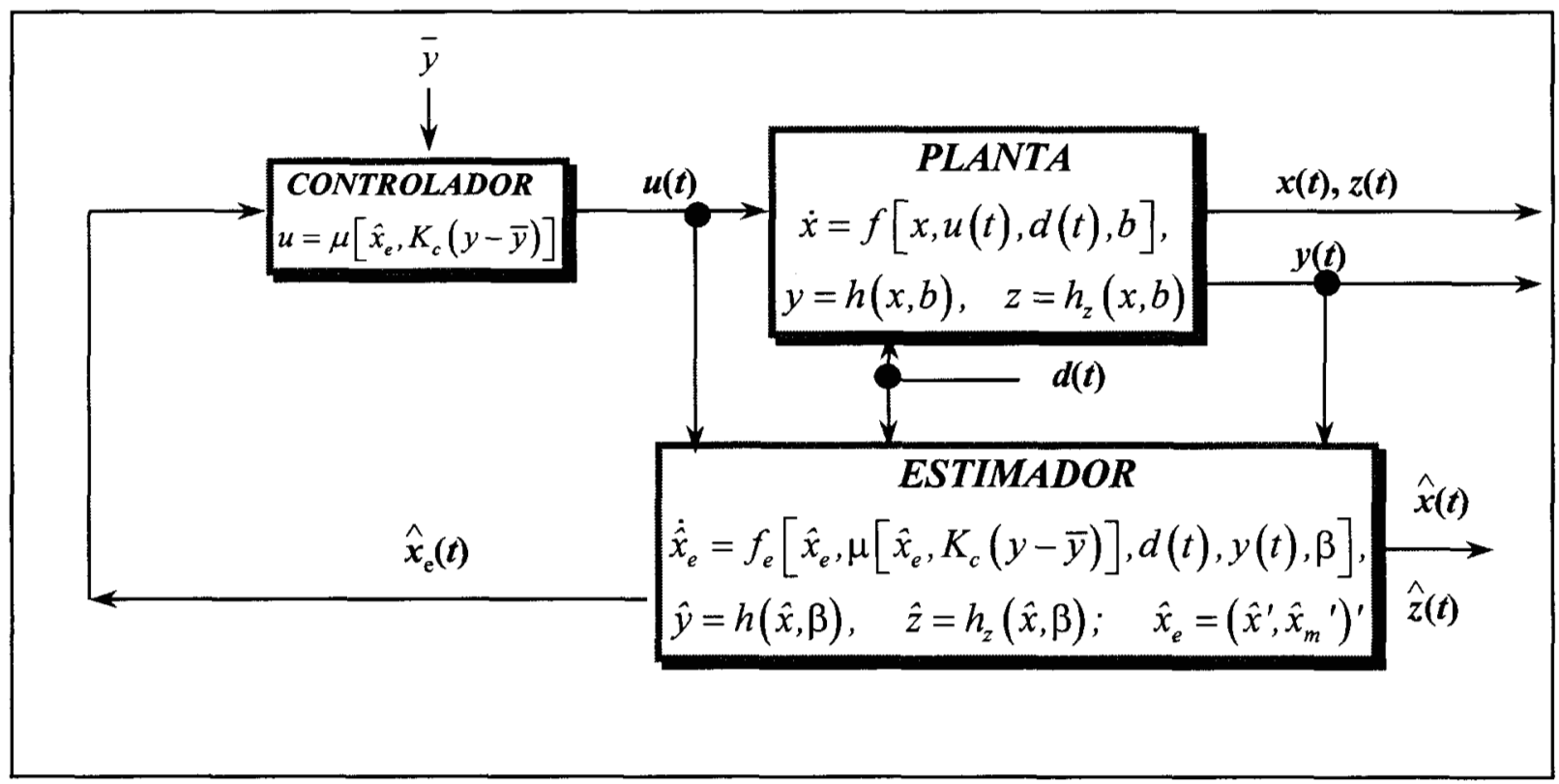

Figura 5.1 Diagrama de bloques del control con retroalimentación de mediciones

Para el caso lineal, el principio de separación (Wonham, 1985) garantiza la estabilidad a lazo cerrado de un controlador con retroalimentación de mediciones, hecho a partir de la interconexión de un controlador con retroalimentación de estados estable con un estimador convergente a lazo abierto. Sin embargo, es bien conocido que, el principio de separación no se cumple de manera directa para el caso no lineal (Vidyasagar, 1978); por tanto, el problema de estabilidad del control no lineal interconectado con un estimador no lineal a lazo cerrado debe garantizarse para su implementación. Puesto que el estimador no lineal ha sido presentado en capítulos anteriores, a continuación se presenta: primero, una sinopsis del control no lineal con retroalimentación de estados (Isidori, 1995), y luego el control no lineal con retroalimentación de mediciones (basado en un estimador no lineal). 


\subsection{Control no lineal co n retroalimentación de estados}

El control con retroalimentación de estados debe considerarse como el "control exacto", es decir, con este control se obtiene el mejor desempeño del sistema a lazo cerrado; bajo la suposición de que todos los estados son conocidos. Este esquema de control ya ha sido estudiado anteriormente (Alvarez, 1996; Padilla y Alvarez, 1997) por lo que en esta sección, el objetivo es hacer referencia a la construcción del control con retoralimentación de estados. Para ello: (i) se define el problema de control con retroalimentación de estados (Isidori, 1989), en la Definición 1; (ii) se establecen sus condiciones de resolubilidad (Alvarez, 1996) en la Definición 2, las cuales también definen la existencia de solución al problema con retroalimentación de mediciones; y (iii) se presenta la construcción y la sintonización del control con retroalimentación de estados en forma condensada en el Teorema 1.

Definición $1 \mathrm{El}$ problema con retroalimentación de estados de la planta (5.1) tiene solución, si existe un controlador no lineal

$$
u=\mu\left[x, K_{c}(y-\bar{y}), b\right]
$$

tal que, en alguna vecindad de la evolución nominal $\bar{E}(t)$ (5.4) (posiblemente inestable), el sistema a lazo cerrado

$$
\dot{x}=f\left\{x, \mu\left[x, K_{c}(y-\bar{y}), b\right], d(t), b\right\}, \quad y=h(x, b), \quad z=h_{z}(x, b)
$$

tiene las siguientes caracteristicas:

(i) $\bar{x}(t)$ es una trayectoria nominal RE-estable, $\mathrm{y}$

(ii) la dinámica del error de salida $\left(\eta_{i}=y_{i}-\bar{y}_{i}\right)$ es LNPA:

$$
\eta_{i}^{\left(\kappa_{i}^{c}\right)}-k_{i \kappa_{i}^{c}}^{c} \eta_{i}^{\left(\kappa_{i}^{c}-1\right)}-\ldots-k_{i 1}^{c} \eta_{i}=0, \quad 1 \leq i \leq m .
$$

Observación 5.1. En general, el problema de control con retroalimentación de estados consiste en obtener las leyes de control, $u(t)$, conociendo todos los estados de la planta; lo cual siempre es factible en la práctica. Mientras que el control con retroalimentación de mediciones se obtiene únicamente a partir de las mediciones. Por ejemplo, en el caso de estudio del reactor de copolimerización, el control con retroalimentación de estados se construirá suponiendo que se miden todos los estados $\left(m_{1}, m_{2}, p_{1}, p_{2}, i, T, V, \mu_{0}, \mu_{2}\right)$, y el control con retroalimentación de mediciones se obtendrá únicamente a partir de $(\rho, \eta, T, \eta)$. No obstante, el mejor (máximo) desempeño obtenido para un control retroalimentado es el control con retroalimentación de estados, y por ello éste se empleará como referencia. 


\subsubsection{Condiciones de resolubilidad}

Para presentar las condiciones de resolubilidad, primero se definirán algunos mapas y vectores, considerando que los parámetros $b$, y las señales $y(t)$ y $d(t)$ son conocidas. Tomemos derivadas temporales sucesivas del mapa de salida $y_{i}=h_{i}(x, b)$ de la ecuación (5.1), hasta el orden $\kappa_{i}^{c}$, obteniendo el conjunto de $\left(\kappa_{c}+m\right)$-ecuaciones (algebraicas variantes en tiempo)

$$
\begin{aligned}
& y(t)=\phi_{I}\left[x, x_{d}(t), b\right] \\
& v(t)=\varphi\left[x, x_{d}(t), b\right]
\end{aligned}
$$

donde $y, x_{d}, \mathrm{y} v$ son vectores obtenidos a partir de las señales medidas $\mathrm{y}$ sus derivadas temporales,

$$
\begin{gathered}
y=\left[y_{1}, \ldots, y_{1}^{\left(\kappa_{1}^{c}-1\right)} ; \ldots ; y_{m}, \ldots, y_{m}^{\left(\kappa_{m}^{c}-1\right)}\right]^{\prime}, \quad v=\left[y_{1}^{\left(\kappa_{1}^{c}\right)}, \ldots, y_{m}^{\left(\kappa_{m}^{c}\right)}\right]^{\prime}, \quad \kappa_{1}^{c}+\ldots+\kappa_{m}^{c}=\kappa_{c} \leq n(5 \\
x_{d}=\left[d_{1}, \ldots, d_{1}^{\left(v_{1}-1\right)} ; \ldots ; d_{p}, \ldots, d_{p}^{\left(v_{p}-1\right)}\right]^{\prime}, \quad v_{1}+\ldots+v_{p}=v
\end{gathered}
$$

y los mapas no lineales $\phi_{I}$ y $\varphi$ están dados por (la derivada direccional recursiva $L_{f}^{i} h$ está definida en 3.13$)$ :

$$
\begin{gathered}
\phi_{l}\left(x, x_{d}, b\right)=\left[h_{1}, \ldots, L_{f}^{\kappa_{1}^{c}-1} h_{1} ; \ldots ; h_{m}, \ldots, L_{f}^{\kappa_{m}^{c}-1} h_{m}\right]^{\prime} \\
\varphi\left(x, u, x_{d}, b\right)=\left[L_{f}^{\kappa_{1}^{c}} h_{1}, \ldots, L_{f}^{\kappa_{m}^{c}} h_{m}\right]^{\prime}
\end{gathered}
$$

En la siguiente definición se establece las condiciones necesarias para obtener el grado relativo de la planta (5.1).

Definición 2 (Grado relativo). La trayectoria nominal de la planta $\bar{x}(t)(5.5)$ tiene un vector de grado relativo

$$
k_{c}=\left(\kappa_{1}^{c}, \ldots, \kappa_{m}^{c}\right), \quad \kappa_{1}^{c}+\ldots+\kappa_{m}^{c}=\kappa_{c} \leq n, \quad \kappa_{i}^{c}>0
$$

si a lo largo de la evolución $\bar{E}(t)(5.4)$ :

(i) Los mapas $\phi_{I}(5.15)$ y $\varphi$ (5.16) son continuamente diferenciales.

(ii) El mapa $\phi_{I}(5.15)$ es independiente de $u$, esto es:

$$
\phi_{I u}\left[\bar{x}(t), \bar{x}_{d}(t), b\right]=0
$$

(iii) El mapa $\phi_{I}(5.15)$ es invertible para $\kappa_{\mathrm{c}}$ estados, esto es:

$$
\text { Rango }\left\{\phi_{I x_{I}}\left[\bar{x}(t), \bar{x}_{d}(t), b\right]\right\}=\kappa \text {. }
$$

(iv) El mapa $\varphi(5.16)$ es $u$-invertible, esto es:

$$
\operatorname{det}\left\{\varphi_{u}\left[\bar{x}, \overline{\mathrm{v}}(t), \bar{x}_{d}(t), b\right]\right\} \neq 0 \quad \text { o } \quad \operatorname{Rango}\left\{\varphi_{u}\left[\bar{x}, \overline{\mathrm{v}}(t), \bar{x}_{d}(t), b\right]\right\}=m
$$


Observación 5.2. La definición anterior establece la existencia de un vector de grados relativos, lo cual garantiza que dadas $m$ salidas $(y)$ es posible reconstruir las $m$ entradas $(u)$ de manera única y controlar $\kappa_{c}$ estados de la planta (estados controlables). El vector de grados relativos $k_{\mathrm{c}}$ se determina derivando sucesivamente las salidas hasta que el mapa $\varphi$ (formado por las $\kappa_{i}^{c}$-ésimas derivadas de las salidas $y_{\mathrm{i}}$ ) sea invertible para las entradas $u, y$ que el mapa $\phi_{\mathrm{I}}$ (formado por las salidas $y_{\mathrm{i}} \mathrm{y}$ sus $\left(\kappa_{\mathrm{i}}^{\mathrm{c}}-1\right)$ derivadas) sea invertible para el mapa $x_{\mathrm{I}}$.

Como consecuencia de la condición (iii) de la Definición 2 , el conjunto de $\kappa_{c}-$ ecuaciones (5.11) admite solución única

$$
x_{I}=\phi_{I}^{-1}\left[x_{I I}, x_{d}(t), y(t), b\right], \quad \operatorname{dim}\left(x_{I}, x_{I I}\right)=\left(\kappa_{c}, n-\kappa_{c}\right)
$$

para $\kappa_{c}$ de los estados del vector $x$, tal que el mapa $\phi_{\mathrm{I}}$ es $x_{\mathrm{I}}$-invertible; esto es,

$$
\phi_{I}^{-1}\left\{x_{I I}, x_{d}(t), \phi_{I}\left[I_{p}\left(x_{I}{ }^{\prime}, x_{I I}{ }^{\prime}\right)^{\prime}, x_{d}(t), b\right], b\right\}=x_{I}, \quad t \geq 0
$$

donde $I_{\mathrm{p}}$ (matriz permutada en columnas de la matriz identidad, de dimensión $n \times n$ ), $x_{\mathrm{I}}$ (partición de estados controlables de dimensión $\kappa_{c}$ ), y $x_{\text {II }}$ (partición de estados no controlables de dimensión $n-\kappa_{c}$ ) cumplen con:

$$
x=I_{p}\left(x_{I}^{\prime}, x_{I I}^{\prime}\right)^{\prime}, \quad\left(x_{I}^{\prime}, x_{I I}\right)^{\prime}=\left(I_{I}, I_{l l}\right) x, \quad\left(I_{l}, I_{I I}\right)=I_{p}^{-1}
$$

Asimismo, la condición (iii) de la Definición 2, existe un mapa complementario $\phi_{\mathrm{II}}$,

$$
x_{I I}=\phi_{I I}(x)
$$

tal que el mapa $\phi$

$$
\phi\left(x, x_{d}, b\right)=\left[\phi_{I}{ }^{\prime}\left(x, x_{d}, b\right), \phi_{I I}{ }^{\prime}(x)\right]^{\prime}
$$

sea $\mathrm{x}$-invertible, esto es:

$$
\phi^{-1}\left[\phi\left(x, x_{d}, b\right), x_{d}, b\right]=x(t)
$$

De igual forma, como consecuencia de la condición (iv) de la Definición 2, el conjunto de $m$-ecuaciones (5.12) admite la solución única

$$
u(t)=\varphi^{-1}\left[x, v(t), x_{d}(t), b\right]
$$

para las $m$-entradas de control, tal que el mapa $\varphi$ es u-invertible; esto es:

$$
\varphi^{-1}\left\{x, \varphi\left[x, u(t), x_{d}(t), b\right], x_{d}(t), b\right\}=u(t)
$$

Por otra parte, la sustituciōn del conjunto de $\kappa_{c}$-ecuaciones (5.11) evaluadas en $\bar{y}(t)$ y del conjunto de $m$-ecuaciones (5.24) evaluadas en $\bar{v}(t)$, en el sistema $n$ dimensional de la planta (5.1) conduce al siguiente sistema algebraico-diferencial: 


$$
\begin{aligned}
\dot{x}_{I I}^{*} & =f_{I I}\left[x_{I I}^{*}, \varphi^{-1}\left[x^{*}, \overline{\mathrm{v}}(t), \bar{x}_{d}(t), b\right], \bar{x}_{d}(t), y(t), b\right], \quad x_{I I}^{*}=x_{I J o}=I_{I I} x_{o} \\
& =f\left[I_{p}^{-1}\left\{\left(\phi_{I}^{-1}\right)^{\prime}\left[x_{I I}^{*}, \bar{x}_{d}(t), y(t), b\right],\left(x_{I I}^{*}\right)^{\prime}\right\}^{\prime}, \varphi^{-1}\left[x^{*}, \overline{\mathrm{v}}(t), \bar{x}_{d}(t), b\right], \bar{x}_{d}(t), b\right] \\
x^{*}(t)= & I_{p}^{-1}\left\{\left(\phi_{I}^{-1}\right)^{\prime}\left[x_{I I}^{*}, x_{d}(t), y(t), b\right],\left(x_{I I}^{*}\right)^{\prime}\right\} \in \Xi(t)=\left\{x \in \mathfrak{R}^{n} \mid y(t)=\phi_{I}\left[x, \bar{x}_{d}(t), b\right]\right\}
\end{aligned}
$$

que producen una trayectoria $x^{*}(t)$ exacta a la trayectoria nominal de la planta $\bar{x}(t)$, siempre y cuando la trayectoria $x_{I I}^{*}$

$x_{I I}^{*}(t)=\theta_{I I}\left[t, t_{o}, \bar{x}_{I l}, \overline{\mathrm{v}}(t), \bar{x}_{d}(t), \bar{y}(t), b\right], \quad x^{*}=\left(x_{I}^{*}, x_{I I}^{*}\right) \in \Xi, \quad 0 \leq \operatorname{dim}\left(x_{I I}^{*}\right) \leq n-\kappa_{c}$

de la dinámica cero (5.26) sea RE-estable.

Observación 5.3. De la ecuación (5.18) puede verse que los $\kappa_{\mathrm{c}}$ estados controlables $x_{1}$ son reconstruidos a partir del mapa controlable $\phi_{1}$, mientras que los $\left(n-\kappa_{\mathrm{c}}\right)$ estados no controlables $x_{11}$ son reconstruidos a partir de su dinámica modelada, llamada dinámica cero. Las entradas $u(t)$ son reconstruidas a partir de la inversión del mapa $\varphi$ (formado por las $\kappa_{i}^{c}$-ésimas derivadas de las salidas $y_{i}$ ), correspondiendo a la inversa dinámica.

\subsubsection{Construcción del controlador}

Como hipótesis, supongamos que: (1) exista un vector $\mathrm{k}$ de grado relativo (de acuerdo a la definición 2), y (2) la dinámica cero (5.27) es RE-estable. Y aplicando el siguiente cambio de coordenadas:

$$
z_{c}=\left[\begin{array}{c}
z_{I}^{c} \\
z_{l l}^{c}
\end{array}\right]:=\left[\begin{array}{c}
\phi_{I}\left(x, x_{d}, b\right) \\
\phi_{I I}(x)
\end{array}\right], \quad \mathrm{v}=\varphi\left(x, u, x_{d}, b\right)
$$

entonces la planta (5.1) en coordenadas- $z$ adquiere la forma normal (Isidori, 1989) (las matrices $\Gamma_{c}, \Pi_{c}, \Delta_{c}$, y el mapa $\theta_{c}$ están definidos en el Apéndice D):

$$
\begin{array}{rlrl}
\dot{z}_{I}^{c} & =\Gamma_{c} z_{I}^{c}+\Pi_{c} \mathrm{v}(t), \quad y=\Delta_{c} z_{I}^{c} ; & \operatorname{dim}\left(z_{I}^{c}\right)=\kappa_{c} \\
\dot{z}_{I I}^{c}=\theta_{c}\left[z_{I}^{c}, z_{I I}^{c}, \cup(t), x_{d}(t), b\right] ; & \operatorname{dim}\left(z_{I I}^{c}\right)=n-\kappa_{c}
\end{array}
$$

donde $z_{I}^{c}$ y $z_{I I}^{c}$ son los estados controlables y no controlables de la planta, respectivamente. Este sistema con $z_{l}^{c}=0$ y $v=0$ corresponde a la dinámica cero (5.26)-(5.27) RE-estable en coordenadas-z. Por otra parte, puesto que el par $\left(\Gamma_{c}, \Pi_{d}\right.$ es controlable, entonces existe una matriz de ganancias, $K_{c}$, tal que el controlador lineal

$$
v=\bar{v}+K_{c}\left(z_{I}^{c}-\bar{z}_{I}^{c}\right)
$$


genera el siguiente sistema a lazo cerrado, sustituyendo (5.31) en (5.30),

$$
\begin{aligned}
& \dot{z}_{I}^{c}=A_{c} z_{I}^{c}+\Pi_{c}\left(\overline{\mathrm{v}}+K_{c} \bar{z}_{I}^{c}\right), \quad y=\Delta_{c} z_{I}^{c}, \quad A_{c}=\Gamma_{c}+\Pi_{c} K_{c} \\
& \dot{z}_{I I}^{c}=\theta_{c}\left[z_{I}^{c}, z_{I I}^{c}, \overline{\mathrm{v}}+K_{c}\left(z_{I}^{c}-\bar{z}_{I}^{c}\right), x_{d}, b\right]
\end{aligned}
$$

El cual será estable si existe una matriz $K_{\mathrm{c}}$ tal que la matriz $A_{c}$ sea estable.

En coordenadas originales, $x=\phi^{-1}\left(z_{c}, x_{d}, b\right)$ (5.29), se obtiene que el controlador no lineal (5.24) está dado por:

$$
u(t)=\varphi^{-1}\left\{x, \overline{\mathrm{v}}+K_{c}\left[\phi_{I}\left(x, x_{d}, b\right)-\phi_{I}\left(\bar{x}, \bar{x}_{d}, b\right)\right], x_{d}(t), b\right\}
$$

y la planta a lazo cerrado (5.32) por:

$$
\begin{aligned}
& \dot{x}=f\left[x, \varphi^{-1}\left\{x, \overline{\mathrm{v}}+K_{c}\left[\phi_{I}\left(x, x_{d}, b\right)-\phi_{I}\left(\bar{x}, \bar{x}_{d}, b\right)\right], x_{d}(t), b\right\}, d(t), b\right], \\
& y=h(x, b), \quad z=h_{z}(x, b)
\end{aligned}
$$

\subsubsection{Dinámica del error y sintonización del controlador}

Definiendo el error de seguimiento/regulación como:

$$
\begin{aligned}
& e_{I}^{c}=z_{I}^{c}-\bar{z}_{I}^{c} \\
& e_{I I}^{c}=z_{I I}^{c}-\bar{z}_{I I}^{c} \\
& e_{d}=\left\|x_{d}(t)-\bar{x}_{d}(t)\right\|^{s}
\end{aligned}
$$

$Y$ restando la ecuación (5.30), evaluada en condiciones nominales, de la ecuación (5.32), se obtiene que la dinámica del error tiene la siguiente forma (los mapas $\omega_{c} y$ $q_{I I}^{c}$ están definidos en el Apéndice D):

$$
\begin{aligned}
& \dot{e}_{I}^{c}=A_{c} e_{I}^{c} \\
& \dot{e}_{I I}^{c}=\omega_{c}\left(e_{I I}^{c}, t\right)+q_{I I}^{c}\left(e_{I}^{c}, e_{I I}^{c}, e_{d}, b, t\right)
\end{aligned}
$$

donde $q_{I l}^{c}$ es una perturbación desvaneciente y L-continua de acuerdo a:

$$
\begin{gathered}
q_{I I}^{c}\left(0, e_{I I}^{c}, 0, b, t\right)=0 \\
\left\|q_{I I}^{c}\left(e_{I}^{c}, e_{I I}^{c}, e_{d}, b, t\right)\right\| \leq N_{I}^{c}\left\|e_{I}^{c}(t)\right\|+N_{d}^{c}\left\|e_{d}(t)\right\|
\end{gathered}
$$


El sistema (5.36) corresponde a un sistema diferencial triangular (Vidyasagar, 1978), el cual es estable si se garantiza que $e_{\mathrm{I}}$ es estable, mediante la selección de una matriz $A_{c}$ estable. Para lo cual sea $K_{c}$ la matriz de ganancias ajustable del controlador de la siguiente forma parametrizada (Alvarez, 1996):

$$
K_{c}\left(s_{c}\right)=d b\left[\left(s_{c}^{\kappa_{1}^{c}} k_{11}^{c}, \ldots, s_{c} k_{1 \kappa_{1}^{c}}^{c}\right)^{\prime}, \ldots,\left(s_{c}^{\kappa_{m}^{c}} k_{m 1}^{c}, \ldots, s_{c} k_{m \kappa_{m}^{c}}^{c}\right)^{\prime}\right], \quad s_{c}>0
$$

con entradas de referencia, $K_{c}(1)$, que hacen estable la dinámica LNPA del error de salida $\left[\eta=y-\bar{y}\right.$, o bien $\eta:=\Delta_{c}\left(z_{I}^{c}-\bar{z}_{l}^{c}\right)$ a partir de la ecuación (5.36)]:

$$
\eta_{i}^{\left(\kappa_{i}^{c}\right)}-k_{i \kappa_{i}^{c}}^{c} \eta_{i}^{\left(\kappa_{i}^{c}-1\right)}-\ldots-k_{i 1}^{c} \eta_{i}=0, \quad \eta_{i}=y_{i}-\bar{y}_{i}, \quad 1 \leq i \leq m
$$

Aqui la sintonización de las ganancias de referencia, $K_{c}(1)$, puede realizarse de forma similar a las ganancias del estimador (Sección3.7.3). Comparando la dinámica (5.39) con la dinámica (3.91) se observa que únicamente difieren en el signo de las ganancias dentro del polinomio característico. Por tanto, de acuerdo al criterio establecido en la Sección 3.7.3, la localización de polos para el controlador es idéntica a la del estimador (ver Figura 3.4); y las ganancias de referencias son las establecidas en la Tabla 5.1.

Tabla 5.1 Ganancias de referencia del controlador

\begin{tabular}{|c|c|}
\hline & \\
\hline 1 & $-\omega_{i}^{c}$ \\
\hline 2 & $-2 \xi \omega_{i}^{c},-\left(\omega_{i}^{c}\right)^{2}$ \\
\hline 3 & $-(2 \xi+1) \omega_{i}^{c},-(2 \xi+1)\left(\omega_{i}^{c}\right)^{2},-\left(\omega_{i}^{c}\right)^{3}$ \\
\hline 4 & $-4 \xi \omega_{i}^{c},-\left(4 \xi^{2}+2\right)\left(\omega_{i}^{c}\right)^{2},-4 \xi\left(\omega_{i}^{c}\right)^{3},-\left(\omega_{i}^{c}\right)^{4}$ \\
\hline 5 & $-(4 \xi+1) \omega_{i}^{c},-\left(4 \xi^{2}+4 \xi+2\right)\left(\omega_{i}^{c}\right)^{2},-\left(4 \xi^{2}+4 \xi+2\right)\left(\omega_{i}^{c}\right)^{3},-(4 \xi+1)\left(\omega_{i}^{c}\right)^{4},-\left(\omega_{i}^{c}\right)^{5}$ \\
\hline
\end{tabular}




\subsubsection{Control a lazo cerrado}

En el siguiente Teorema se resume la construcción y sintonización del control con retroalimentación de estados, recordando que la estabilidad del sistema a lazo cerrado se ha garantizado con las dos suposiciones hechas (existencia de un vector de grado relativo y estabilidad de la dinámica cero).

Teorema 1 (Control con retroalimentación de estados). Sea la planta (5.1) con grado relativo $k=\left(\kappa_{1}^{c}, \ldots, \kappa_{m}^{c}\right.$ ) (de acuerdo a la Definición 2) y con dinámica cero (5.28) REestable, y con ganancias parametrizadas

$$
K_{c}\left(s_{c}\right)=d b\left[\left(s_{c}^{\kappa_{1}^{c}} k_{11}^{c}, \ldots, s_{c} k_{1 \kappa_{1}^{c}}^{c}\right)^{\prime}, \ldots,\left(s_{c}^{\kappa_{m}^{c}} k_{m 1}^{c}, \ldots, s_{c} k_{m \kappa_{m}^{c}}^{c}\right)^{\prime}\right], \quad s_{c}>0
$$

Entonces, el controlador no lineal

$$
u(t)=\varphi^{-1}\left\{x, \overline{\mathrm{v}}+K_{c}\left[\phi_{I}\left(x, x_{d}, b\right)-\phi_{I}\left(\bar{x}, \bar{x}_{d}, b\right)\right], x_{d}(t), b\right\}:=u^{o}(t)
$$

genera un sistema a lazo cerrado

$$
\begin{aligned}
& \dot{x}=f\left[x, \varphi^{-1}\left\{x, \overline{\mathrm{v}}+K_{c}\left[\phi_{I}\left(x, x_{d}, b\right)-\phi_{I}\left(\bar{x}, \bar{x}_{d}, b\right)\right], x_{d}(t), b\right\}, d(t), b\right], \\
& y=h(x, b), \quad z=h_{z}(x, b)
\end{aligned}
$$

en el cual:

(i) la trayectoria $x(t)$ converge- $\mathrm{E}$ a la trayectoria nominal $\bar{x}(t)$.

(ii) la salida $y(t)$ converge-E a la salida nominal $\bar{y}(t)$ con dinámica LNPA.

$$
\eta_{i}^{\left(\kappa_{i}^{c}\right)}-k_{i \kappa_{i}^{c}}^{c} \eta_{i}^{\left(\kappa_{i}^{c}-1\right)}-\ldots-k_{i 1}^{c} \eta_{i}=0, \quad \eta_{i}=y_{i}-\bar{y}_{i}, \quad 1 \leq i \leq m
$$

donde las ganancias de referencia corresponden a $K_{c}(1)=d b\left[\left(k_{11}^{c}, \ldots, k_{1 \mathrm{k}_{1}^{c}}^{c}\right)^{\prime}, \ldots\right.$, $\left.\left(k_{m 1}^{c}, \ldots, k_{m \kappa_{m}^{c}}^{c}\right)^{\prime}\right]$.

(iii) la entrada $u(t)$ converge- $\mathrm{E}$ a la entrada nominal $\bar{u}(t)$.

Observación 5.4. El controlador $\mathfrak{u}^{\circ}(t)$ (5.41) corresponde al "controlador exacto". Esto es, este controlador con retroalimentación de estados (sin errores de modelado, ni ruido en las mediciones) generará el máximo desempeño alcanzable y la máxima velocidad de convergencia. Por esto, este controlador se usará como referencia para comparar el control no lineal basado en un estimador (i.e, con retroalimentación de mediciones) que se propone en las siguientes secciones. 


\subsection{Control no lineal ba sado en un estimador}

\subsubsection{Construcción del controlador}

Consideremos que el problema de control con retroalimentación de estados tiene solución de acuerdo al Teorema 2, y que la planta es RE-estimable de acuerdo a la Definición 2 (Capitulo 3), con grados relativos igual a los indices de observabilidad:

$$
\left(\kappa_{1}^{c}, \ldots, \kappa_{m}^{c}\right)=\left(\kappa_{1}, \ldots, \kappa_{m}\right), \quad \kappa_{c}=\kappa
$$

Reescribamos el estimador-PI (3.78) a lazo abierto con las siguientes dos modificaciones: (1) el vector de entradas exógenas $x_{u}$ del estimador ahora corresponde al vector $x_{d}$ (las matrices $K_{d}, \Gamma_{d}$ y $\Delta_{d}$ son iguales a $K_{u}, \Gamma_{u}$ y $\Delta_{u}$ ), e (2) incluyamos la acción integral como un estado adicional, $x_{q}$. Esto es:

$$
\begin{aligned}
& \dot{\hat{x}}_{d}=\Gamma_{d} \hat{x}_{d}+K_{d}\left(s_{d}\right)\left(d-\Delta_{d} \hat{x}_{d}\right), \quad \hat{d}=\Delta_{d} \hat{x}_{d} \\
& \dot{\hat{x}}_{q}=K_{I}\left(s_{o}\right)[y-h(\hat{x}, \beta)] \\
& \dot{\hat{x}}=f\left[\hat{x}, u(t), \Delta_{d} \hat{x}_{d}, \beta\right]+G\left(\hat{x}, \hat{x}_{d}, \beta\right)[y-h(\hat{x}, \beta)]+H\left(\hat{x}, \hat{x}_{d}, \beta\right) \hat{x}_{q} \\
& \hat{y}=h(\hat{x}, \beta), \quad \hat{z}=h_{z}(\hat{x}, \beta) \\
& G\left(\hat{x}, \hat{x}_{d}, \beta\right)=\left[I_{11}^{\prime}, I_{12}^{\prime}\right]^{\prime} \phi_{I x_{I}}^{-1}\left(\hat{x}, \hat{x}_{d}, \beta\right) K_{o}\left(s_{o}\right) ; \\
& H\left(\hat{x}, \hat{x}_{d}, \beta\right)=\left[I_{11}^{\prime}, I_{12}^{\prime}\right]^{\prime} \phi_{I x_{l}}^{-1}\left(\hat{x}, \hat{x}_{d}, \beta\right) \Pi_{o}
\end{aligned}
$$

En el cual $\hat{x}_{q}$ (o bien $z_{\mathrm{q}}$ en la ecuación 3.63) corresponde a la estimación de la parte lenta de la perturbación persistente de la dinámica observable, debido principalmente a errores en los parámetros del modelo; y converge asintóticamente a (ver ecuación 3.61, en coordenadas originales):

$$
\hat{x}_{q} \rightarrow x_{q}=\varphi\left[x, u(t), x_{d}(t), b\right]-\varphi\left[\hat{x}, u(t), \hat{x}_{d}(t), \beta\right]
$$

Asimismo el error observable (3.68) converge-RE a cero, de acuerdo a:

$$
e_{I}(t)=\phi_{I}\left[\hat{x}, \hat{x}_{d}(t), \beta\right]-\phi_{I}\left[x, x_{d}(t), b\right] \rightarrow 0
$$

Supongamos que la planta es sujeta al controlador exacto (5.42) con retroalimentación de estados, y que el estimador-PI (5.45) a lazo abierto sujeto a la entrada $u(t)=u^{\circ}(t)(5.41)$ es RE-convergente, tal que:

y

$$
\begin{aligned}
\hat{x}_{q} \rightarrow x_{q}= & \varphi\left\{\bar{x}, \overline{\mathrm{v}}+K_{c}\left[\phi_{I}\left(x, x_{d}, b\right)-\phi_{I}\left(\bar{x}, \bar{x}_{d}, b\right)\right], \bar{x}_{d}(t), b\right\}- \\
& \varphi\left\{\hat{x}, \overline{\mathrm{v}}+K_{c}\left[\phi_{I}\left(x, x_{d}, b\right)-\phi_{I}\left(\bar{x}, \bar{x}_{d}, b\right)\right], \hat{x}_{d}(t), \beta\right\}
\end{aligned}
$$

$$
e_{I}(t)=\phi_{I}\left[\hat{x}, \hat{x}_{d}(t), \beta\right]-\phi_{I}\left[\bar{x}, \bar{x}_{d}(t), b\right] \rightarrow 0
$$


Ya que el mapa $\varphi(5.16)$ es Ru-invertible (de acuerdo a la condición iv de la Definición $2)$, entonces existe una función $x_{u}(t)$ tal que el estimador basado en el control $u(t)$

$$
u(t)=\varphi^{-1}\left\{\hat{x}, \overline{\mathrm{v}}+K_{c}\left[\phi_{I}\left(\hat{x}, \hat{x}_{d}, \beta\right)-\phi_{I}\left(\bar{x}, \bar{x}_{d}, b\right)\right], \hat{x}_{d}(t), \beta\right\}
$$

converge al exacto $u^{\circ}(t)$

$$
u^{o}(t)=\varphi^{-1}\left\{x, \overline{\mathrm{v}}+K_{c}\left[\phi_{I}\left(x, x_{d}, b\right)-\phi_{I}\left(\bar{x}, \bar{x}_{d}, b\right)\right], x_{d}(t), b\right\}
$$

Por tanto la función $x_{u}(t)$ está dada por la diferencia entre estas dos ecuaciones:

$$
\begin{aligned}
x_{u}(t)= & \varphi^{-1}\left\{x, \overline{\mathrm{v}}+K_{c}\left[\phi_{I}\left(x, x_{d}, b\right)-\phi_{I}\left(\bar{x}, \bar{x}_{d}, b\right)\right], x_{d}(t), b\right\}- \\
& \varphi^{-1}\left\{\hat{x}, \overline{\mathrm{v}}+K_{c}\left[\phi_{I}\left(\hat{x}, \hat{x}_{d}, \beta\right)-\phi_{I}\left(\bar{x}, \bar{x}_{d}, b\right)\right], \hat{x}_{d}(t), \beta\right\}
\end{aligned}
$$

o bien, de acuerdo a (5.48) y (5.49)

$$
x_{u}(t)=x_{q}(t)-e_{I}(t)
$$

Ya que $e_{I} \rightarrow 0$, entonces el estimado $\hat{x}_{q}(t)$ converge asintóticamente a la función de control $x_{u}(t)$, implicando que el controlador basado en el estimador está dado por:

$$
u(t)=\varphi^{-1}\left\{\hat{x}, \overline{\mathrm{U}}+K_{\mathrm{c}}\left[\phi_{l}\left(\hat{x}, \hat{x}_{d}, \beta\right)-\phi_{l}\left(\bar{x}, \bar{x}_{d}, b\right)\right]-\hat{x}_{q}, \hat{x}_{d}(t), \beta\right\}:=\gamma\left(\hat{x}, \hat{x}_{q}, \hat{x}_{d}, \beta\right)
$$

para el cual $\hat{x}_{q}(t) \rightarrow x_{u}(t)$, y el controlador $u(t) \rightarrow u^{\circ}(t)$.

\subsubsection{Control a lazo cerrado y dinámica del error}

La combinación de este controlador (5.54) (reconstructor del control con retroalimentación de estados) con el estimador (5.45) a lazo abierto, genera un controlador "candidato" forzado por las salidas

$$
\begin{aligned}
& \dot{\hat{x}}_{d}=\Gamma_{d} \hat{x}_{d}+K_{d}\left(s_{d}\right)\left(d-\Delta_{d} \hat{x}_{d}\right) \\
& \dot{\hat{x}}_{q}=K_{I}\left(s_{o}\right)[y-h(\hat{x}, \beta)] \\
& \dot{\hat{x}}=f\left[\hat{x}, \gamma\left(\hat{x}, \hat{x}_{q}, \hat{x}_{d}, \beta\right), \Delta_{d} \hat{x}_{d}, \beta\right]+G\left(\hat{x}, \hat{x}_{d}, \beta\right)[y-h(\hat{x}, \beta)] \\
& \hat{y}=h(\hat{x}, \beta), \quad \hat{z}=h_{z}(\hat{x}, \beta) ; \quad u(t)=\gamma\left(\hat{x}, \hat{x}_{q}, \hat{x}_{d}, \beta\right)
\end{aligned}
$$

donde el término $H\left(\hat{x}, \hat{x}_{d}, \beta\right) \hat{x}_{q}$ de la dinâmica del estimador a lazo abierto (5.45) se ha cancelado, en consonancia con la idea de compensar y rechaza los errores persistentes de modelado en el sistema a lazo cerrado. 
La planta a lazo cerrado

$$
\begin{aligned}
& \dot{\hat{x}}_{d}=\Gamma_{d} \hat{x}_{d}+K_{d}\left(s_{d}\right)\left(d-\Delta_{d} \hat{x}_{d}\right) \\
& \dot{\hat{x}}_{q}=K_{I}\left(s_{o}\right)[y-h(\hat{x}, \beta)] \\
& \dot{\hat{x}}=f\left[\hat{x}, \gamma\left(\hat{x}, \hat{x}_{q}, \hat{x}_{d}, \beta\right), \Delta_{d} \hat{x}_{d}, \beta\right]+G\left(\hat{x}, \hat{x}_{d}, \beta\right)[y-h(\hat{x}, \beta)] ; \hat{y}=h(\hat{x}, \beta), \quad \hat{z}=h_{z}(\hat{x}, \beta) \\
& \dot{x}=f\left[x, \gamma\left(\hat{x}, \hat{x}_{q}, \hat{x}_{d}, \beta\right), d(t), b\right], \quad y=h(x, b), \quad z=h_{z}(x, b)
\end{aligned}
$$

con ganancias parametrizadas

$$
\begin{aligned}
& K_{d}\left(s_{d}\right)=d b\left[\left(s_{d} k_{11}^{d}, \ldots, s_{d}^{v_{1}} k_{v_{1} 1}^{d}\right)^{\prime}, \ldots,\left(s_{d} k_{1 p}^{d}, \ldots, s_{d}^{v_{p}} k_{v_{p} p}^{d}\right)^{\prime}\right], \quad s_{d}>0 \\
& K_{o}\left(s_{o}\right)=d b\left[\left(s_{o} k_{11}^{o}, \ldots, s_{o}^{\kappa_{1}} k_{\kappa_{1} 1}^{o}\right)^{\prime}, \ldots,\left(s_{o} k_{1 m}^{o}, \ldots, s_{o}^{\kappa_{m}} k_{\kappa_{m} m}^{o}\right)^{\prime}\right], \quad s_{o}>0 \\
& K_{l}\left(s_{o}\right)=\operatorname{diag}\left[s_{o}^{\mathrm{\kappa}_{1}+1} k_{1}^{I}, \ldots, s_{o}^{\mathrm{K}_{m}+1} k_{\kappa_{m}}^{I}\right] \\
& K_{c}\left(s_{c}\right)=d b\left[\left(s_{c}^{\kappa_{1}^{c}} k_{11}^{c}, \ldots, s_{c} k_{1 \kappa_{1}^{c}}^{c}\right)^{\prime}, \ldots,\left(s_{c}^{\mathbf{\kappa}_{m}^{c}} k_{m 1}^{c}, \ldots, s_{c} k_{m \kappa_{m}^{c}}^{c}\right)^{\prime}\right], \quad s_{c}>0
\end{aligned}
$$

y entradas de referencia, $\left\{K_{d}(1), K_{o}(1), K_{f}(1), K_{c}(1)\right\}$, que hacen estable las dinâmicas LNPA del error

$$
\begin{array}{lrl}
\mu_{i}^{\left(v_{i}\right)}+k_{1 i}^{d} \mu_{i}^{\left(v_{i}-1\right)}+\ldots+k_{v_{i} i}^{d} \mu_{i}=0, & 1 \leq i \leq p ; & \mu_{i}=\hat{d}_{i}-d_{i} \\
v_{i}^{\left(\kappa_{i}+1\right)}+k_{1 i}^{o} v_{i}^{\left(\kappa_{i}\right)}+\ldots+k_{\kappa_{i} i}^{o} \dot{v}_{i}+k_{i}^{l} v_{i}=0, & 1 \leq i \leq m ; & v_{i}=\hat{y}_{i}-y_{i} \\
\eta_{i}^{\left(\kappa_{i}^{c}\right)}-k_{i \kappa_{i}^{c}}^{c} \eta_{i}^{\left(\kappa_{i}^{c}-1\right)}-\ldots-k_{i 1}^{c} \eta_{i}=0, & 1 \leq i \leq m ; & \eta_{i}=y_{i}-\bar{y}_{i}
\end{array}
$$

produce una evolución $E_{c}(t)$ que converge-RE a la nominal $\bar{E}(t)$ (5.4) de acuerdo al siguiente teorema [el análisis de estabilidad y la prueba de la condición de convergencia son temas de investigación fuera del alcance de esta tesis, y actualmente se encuentran en estudio (Alvarez et al., 2000a, 2000b)]

Teorema 2 (Control con retroalimentación de mediciones). Sea el problema de control con retroalimentación de estados de la planta (5.1) resoluble de acuerdo al Teorema 1, y sea la planta RE-estimable de acuerdo a la Definición 2; con grados relativos iguales a los indices de observabilidad

$$
\kappa_{1}+\ldots+\kappa_{m}=\kappa \leq n, \quad \kappa_{i}^{c}=\kappa_{i}>0
$$


Entonces, el controlador no lineal (5.54) genera un sistema a lazo cerrado (5.56), en el cual las trayectorias estimadas convergen-RE a

(i) $\hat{x}(t) \rightarrow x(t) \rightarrow \bar{x}(t)$

(ii) $\hat{y}(t) \rightarrow y(t) \rightarrow \bar{y}(t)$

(iii) $u(t) \rightarrow u^{o}(t) \rightarrow \bar{u}(t)$

Si los parámetros $\left(s_{0}, s_{\mathrm{d}}\right)$ del estimador son escogidos suficientemente grandes (rápidos), tal que se cumplan las siguientes desigualdades:

$$
\begin{gathered}
l_{d}>l_{o}>0 \\
l_{d}:=s_{d} \lambda_{d}, \quad l_{o}:=s_{o} \lambda_{o}-a_{o}\left[l_{a}^{\theta_{a}}+\left(a_{x} / \lambda_{x}\right) l_{I I}^{\theta_{a}} l_{I}^{q_{I I}}\right] .
\end{gathered}
$$

Observación 5.5. La condición de convergencia (5.60) indica que el exo-observador (Filtro para las entradas exógenas, $d$ ) es más rápido que el estimador, y éste a su vez que el controlador: $s_{\mathrm{d}}>s_{\mathrm{o}}>s_{\mathrm{c}}$. Siendo la compensación de errores de modelado $\left(x_{\mathrm{q}}\right)$ rápida, y por tanto hace que el controlador compense tambiên rápidamente estos errores. Asimismo, el control a lazo cerrado converge si el margen de estabilización $l_{o}=s_{o} \lambda_{o}$ se hace más grande el término $a_{o}\left[l_{a}^{\theta_{a}}+\left(a_{x} / \lambda_{x}\right) l_{l l}^{\theta_{a}} l_{I}^{q_{I}}\right]$, el cual toma en cuenta un potencial de auto-desestabilización de la dinámica cero, y de desestabilización debido al acoplamiento entre la dinámica controlable y la dinámica cero.

\subsection{Control de los react ores continuos de copolimerización en solución}

Para el diseño e implementación del esquema de control para el reactor continuo de copolimerización de MMA-AV en solución, recordemos que este reactor a lazo abierto tiene tres estados estacionarios (ver Capítulo 2): un punto de ignición (estable, con alta fracción de sólidos y alta temperatura), un punto de extinción (estable, con baja fracción de sólidos y baja temperatura), y un punto intermedio (inestable, con fracción de sólidos y temperatura intermedios). Siendo el punto de interés o punto de referencia para regulación, el punto inestable intermedio.

Por lo anterior, en esta sección el objetivo es establecer un esquema de control no lineal con retroalimentación de mediciones, para regular las variables de calidad (Composición, conversión, y peso molecular peso-promedio) y la velocidad de producción del reactor en el estado estacionario intermedio (inestable a lazo abierto, 
ver Tabla 2.4). Seleccionando las variables de control como: los flujos de alimentación de los monómeros 1 y 2 , la temperatura de la camisa de enfriamiento, y el flujo de descarga,

$$
u=\left(q_{1}, q_{2}, T_{c}, q\right)^{\prime}
$$

y conociendo las entradas exógenas (constantes o variantes en tiempos): flujos y temperaturas de alimentación de los monómeros, del solvente, y del iniciador,

$$
d(t)=\left[\begin{array}{c}
d_{1}(t) \\
d_{2}
\end{array}\right], \quad\left\{\begin{array}{l}
d_{1}(t)=\left(m_{1 e}, T_{1 e}, T_{2 e}, T_{s e}\right)^{\prime} \\
d_{2}=\left(m_{2 e}, p_{1 e}, p_{2 e}, q_{s}, w_{l e}\right)^{\prime}
\end{array}\right.
$$

Las salidas de control $(y)$ se definen para dos casos:

A. Control con retroalimentación de mediciones, definido como $C_{\mathrm{y}}$. Donde se consideran las mediciones indirectas de densidad, indice de refracción, temperatura y volumen

$$
y=\left(y_{\rho}, y_{\eta}, y_{T}, y_{V}\right)^{\prime}
$$

y cuyo desempeño seria el esperado en situaciones prácticas industriales.

B. Control con retroalimentación de estados, definido como $C_{\mathbf{x}}$. Donde se consideran las mediciones directas de cuatro estados en el reactor: concentraciones de los dos monómeros remanentes, temperatura, y volumen

$$
y=\left(y_{m_{1}}, y_{m_{2}}, y_{T}, y_{V}\right)^{\prime}
$$

Este controlador se implementará con la finalidad de obtener el máximo desempeño del controlador (exacto), y tener un comparativo para el control $C_{\mathrm{y}}$ (recordando que el caso $C_{\mathrm{x}}$ no es de interés práctico, ya que no se conocen los estados sino mediciones indirectas de ellos).

A continuación se estudia el esquema de control geométrico no lineal, nuevamente en dos etapas: una etapa de análisis, donde se define la estructura de estimabilidadcontrolabilidad, así como las condiciones para la existencia de solución al problema de control; y una segunda etapa de diseño, donde se aplica directamente el Teorema 2 para su construcción-sintonización, y finalmente se implementa el controlador. 


\subsubsection{Estructura del control}

\section{A. Control con retroalimentación de mediciones}

De acuerdo al Teorema 2, el problema de control con retroalimentación de mediciones tiene solución si el reactor: (1) es RE-estimable, de acuerdo a la Definición 2 - Capitulo $3,(2)$ tiene grado relativo $k=\left(\kappa_{1}^{c}, \ldots, \kappa_{m}^{c}\right)$, de acuerdo a la Definición 2, y (3) la dinámica cero (5.28) es RE-estable, y (4) los grados relativos son iguales a los indices de observabilidad (5.59).

Del análisis de estimabilidad (Capítulo 4) se concluyó que las tres mejores estructuras, $S=\left(k, x_{I}\right)$, son las siguientes:

$1^{\mathrm{a}} . \quad S_{3}=\left\{(2,2,1,1),\left(m_{1}, m_{2}, p_{1}, p_{2}, T, V\right)\right\} \in \Sigma_{3}$

$2^{\mathrm{a}} . \quad S_{2}=\left\{(2,1,1,1),\left(m_{1}, m_{2}, p_{2}, T, V\right)\right\} \in \Sigma_{2}$

$3^{\text {a }} . S_{l}=\left\{(1,1,1,1),\left(m_{1}, m_{2}, T, V\right)\right\} \in \Sigma_{1}$

Asimismo de dicho análisis se concluyó que los mapas $\phi_{1}$ y $\varphi$ son continuamente diferenciables [condición (i) de la Definición 2]. Sin embargo, para fines de control, las estructuras $S_{2}$ y $S_{3}$ no cumplen con la condición (ii) de la Definición 2, ya que el mapa $\phi_{I}$ para estas dos estructuras no es independiente del vector de entradas $u$ :

$$
\begin{gathered}
S_{2}: \quad \phi_{I}[x, u(t), d(t), b]=\left[\rho(x), \rho_{x}(x) f[x, u(t), d(t), b], \eta(x), x_{6}, x_{7}\right] \\
S_{3}: \quad \phi_{I}[x, u(t), d(t), b]=\left[\rho(x), \rho_{x}(x) f[x, u(t), d(t), b], \eta(x), \eta_{x}(x) f[x, u(t), d(t), b], x_{6}, x_{7}\right]
\end{gathered}
$$

Mientras que la estructura $S_{1}$ satisface todas las condiciones de la definición de grado relativo(Definición 2). Esto es, el reactor con la estructura $S_{1}$ tiene un vector de grado relativo

$$
k=\left(\kappa_{1}, \kappa_{2}, \kappa_{3}, \kappa_{4}\right)=(1,1,1,1), \quad \kappa=\kappa_{c}=4
$$

con el cual, los mapas $\phi_{I}$ y $\varphi$ están dados por:

$$
\begin{gathered}
\phi_{l}(x, b)=\left[\rho(x), \eta(x), x_{6}, x_{7}\right]^{\prime} \\
\varphi[x, u(t), d(t), b]=\left[\rho_{x}(x) f(x, u, d, b), \eta_{x}(x) f(x, u, d, b), f_{6}, f_{7}\right]^{\prime}
\end{gathered}
$$

y se cumple que,

(i) Los mapas $\phi_{I}(5.66)$ y $\varphi$ (5.67) son continuamente diferenciales.

(ii) $\mathrm{El}$ mapa $\phi_{I}(5.66)$ es independiente de $u$, esto es: $\phi_{h u}(\bar{x}, b)=0$.

(iii) El mapa $\phi_{I}(5.66)$ tiene Rango $\left[\phi_{I x_{I}}(\bar{x}, b)\right]=\kappa=4$.

(iv) El mapa $\varphi$ (5.67) tiene Rango $\left\{\varphi_{u}\left[\bar{x}, \bar{u}(t), \bar{x}_{d}(t), b\right]\right\}=m=4$. 
Además para esta estructura, la partición de estados controlables - no controlables están dados por:

$$
x_{l}=\left(m_{1}, m_{2}, T, V\right)^{\prime}, \quad x_{I I}=\phi_{I I}(x):=\left(p_{l}, p_{2}, i, \mu_{0}, \mu_{2}\right)^{\prime}
$$

con lo cual el mapa de controlabilidad $\phi$

$$
\phi[x, d(t), b]=\left\{\phi_{I}[x, d(t), b]^{\prime}, \phi_{I I}(x)^{\prime}\right\}^{\prime}
$$

es invertible para el vector de estados $x$.

Por otra parte, el mapa $\varphi(5.67)$ puede reescribirse como

$$
\varphi[x, u(t), d(t), b]:=v(t)=C[x, d(t), b]+A[x, d(t), b] u(t)
$$

donde $\left(f_{r i}\right.$ y $g_{r i j}$ están definidos en el Apéndice A):

$$
\begin{gathered}
\mathrm{v}(t)=\left(\dot{y}_{\rho}, \dot{y}_{\eta}, \dot{y}_{T}, \dot{y}_{V}\right)^{\prime} \\
C[x, d(t), b]=\left[\sum_{i=1}^{9} \rho_{x_{i}} f_{r i}, \sum_{i=1}^{9} \eta_{x_{i}} f_{r i}, f_{r 6}, f_{r 7}\right], \\
A[x, d(t), b]=\left[\begin{array}{lllc}
\sum_{i=1}^{9} \rho_{x_{i}} g_{r 1 i} & \sum_{i=1}^{9} \rho_{x_{i}} g_{r 2 i} & 0 & 0 \\
\sum_{i=1}^{9} \eta_{x_{i}} g_{r 1 i} & \sum_{i=1}^{9} \eta_{x_{i}} g_{r 2 i} & 0 & 0 \\
g_{r 16} & g_{r 26} & g_{r 36} & 0 \\
g_{r 17} & g_{r 27} & 0 & g_{r 47}
\end{array}\right]
\end{gathered}
$$

tal que, de acuerdo a la condición (iv) de la definición de grado relativo, el mapa $\varphi$ es invertible para el vector de entradas $u(t)$ :

$$
u(t)=\varphi^{-1}[x, v(t), d(t), b]:=A[x, d(t), b]^{-1}\{v(t)-C[x, d(t), b]\}
$$

Por último, la dinámica cero del reactor corresponde a la dinámica de los estados $x_{I}$ (5.68) evaluada con

$$
x_{I}=\phi_{I}^{-1}\left[x_{l l}, \bar{d}(t), \bar{y}(t), b\right], \quad \mathrm{y} \quad u(t)=A[x, d(t), b]^{-1}\{\bar{v}(t)-C[x, d(t), b]\}
$$

siendo $\bar{v}(t)=0$ para regulación de un punto de referencia (que corresponde a nuestro caso de estudio). Por consiguiente la dinámica cero está dada por:

$$
\dot{x}_{I I}^{*}=\left\{f_{I I}[x, d(t), b]-g_{I I}[x, d(t), b] A[x, d(t), b]^{-1} C[x, d(t), b]\right\}_{x=\left(x_{I}, x_{H}\right)}
$$

donde $\quad x_{I I}^{*}=\left(p_{1}^{*}, p_{2}^{*}, i^{*}, \mu_{0}^{*}, \mu_{2}^{*}\right)^{\prime}, \quad f_{I I}=\left(f_{r 3}, f_{r 4}, f_{r 5}, f_{r 8}, f_{r 9}\right)^{\prime}, \quad g_{I I}=\left(g_{r 3}, g_{r 4}, g_{r 5}, g_{r 8}, g_{r 9}\right)^{\prime}$

La prueba de la estabilidad de la dinámica cero (5.75) consisten en verificar que el estado estacionario $\bar{x}$ es un punto crítico único y estable. Esto se corroboró mediante un diagrama de bifurcación (con el parámetro de bifurcación $\alpha$ definido en 
la ecuación 2.15). Como puede verse en la Figura 5.2, la dinámica cero tiene un solo estado estacionario estable, correspondiendo $\alpha=1$ al estado estacionario $\bar{x}$ de interés. Por tanto se concluye que la dinámica cero es RE-estable.

En resumen, el problema de control con retroalimentación de mediciones tiene solución para el conjunto de salidas $y=\left(y_{\rho}, y_{\eta}, y_{T}, y_{V}\right)^{\prime}$, con grados relativos $k=(1,1$, $1,1)$ y dinámica cero $(5.75)$ RE-estable.

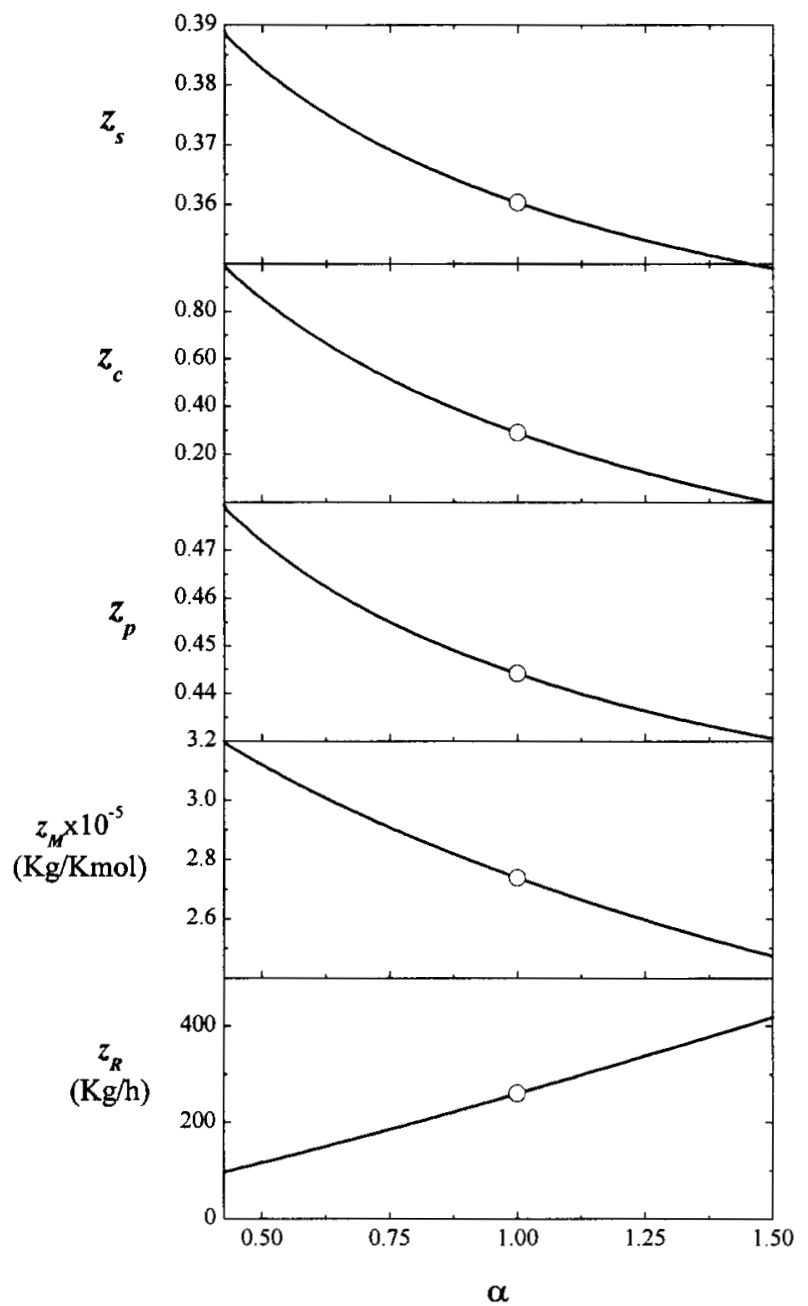

Figura 5.2 Diagrama de bifurcación de la dinámica cero del reactor. 


\section{B. Control con retroalimentación de estados}

Como se mencionó anteriormente, el caso con retroalimentación de estados se implementará con fines de comparación. Por lo cual aquî se selecciona el mapa de salidas igual al vector $x_{\mathrm{I}}(5.68)$ del esquema de control con retroalimentación de mediciones, esto es:

$$
y=\left(y_{m_{1}}, y_{m_{2}}, y_{T}, y_{V}\right)^{\prime}, \quad h(x, b)=\left(m_{1}, m_{2}, T, V\right)^{\prime}
$$

Para este conjunto de salidas y con grados relativos

$$
k=\left(\kappa_{1}, \kappa_{2}, \kappa_{3}, \kappa_{4}\right)=(1,1,1,1), \quad \kappa=\kappa_{c}=4
$$

los mapas $\phi_{I}$ y $\varphi$ están dados por

$$
\begin{gathered}
\phi_{I}(x)=\left(x_{1}, x_{2}, x_{6}, x_{7}\right)^{\prime} \\
\varphi[x, u(t), d(t), b]=\left(f_{1}, f_{2}, f_{6}, f_{7}\right)_{[x, u(t), d(t), b]}^{\prime}
\end{gathered}
$$

Con lo cual se satisfacen todas las condiciones de la definición de grado relativo (Definición 2), esto es:

(i) Los mapas $\phi_{I}(5.78)$ y $\varphi(5.79)$ son continuamente diferenciales.

(ii) El mapa $\phi_{I}(5.78)$ es independiente de $u$, esto es: $\phi_{l u}(\bar{x})=0$.

(iii) El mapa $\phi_{I}(5.78)$ tiene Rango $\left[\phi_{l x_{I}}(\bar{x})\right]=\kappa=4$.

(iv) El mapa $\varphi$ (5.79) tiene Rango $\left\{\varphi_{u}\left[\bar{x}, \bar{u}(t), \bar{x}_{d}(t), b\right]\right\}=m=4$.

Aquí la partición de estados controlables $\left(x_{\mathrm{I}}\right)$ - no controlables $\left(x_{\mathrm{II}}\right)$ es igual al esquema anterior (5.68). Y el mapa de controlabilidad está definido por:

$$
\phi(x)=\left[\phi_{I}(x)^{\prime}, \phi_{I I}(x)^{\prime}\right]^{\prime}:=\left[\left(m_{1}, m_{2}, T, V\right)^{\prime},\left(p_{1}, p_{2}, i, \mu_{0}, \mu_{2}\right)^{\prime}\right]^{\prime}
$$

cuyo jacobiano $\phi_{\mathrm{x}}$ es igual a la identidad permutada, y por tanto $\phi$ es invertible para el vector de estados $x$.

El mapa $\varphi$ (5.79) puede reescribirse como

$$
\varphi[x, u(t), d(t), b]:=v(t)=C[x, d(t), b]+A[x, d(t), b] u(t)
$$

donde $\left(f_{r i}\right.$ y $g_{r i j}$ están definidos en el Apéndice A):

$$
\begin{gathered}
v(t)=\left(\dot{y}_{\rho}, \dot{y}_{\eta}, \dot{y}_{T}, \dot{y}_{V}\right)^{\prime} \\
C[x, d(t), b]=\left[f_{r 1}, f_{r 2}, f_{r 6}, f_{r 7}\right]^{\prime}
\end{gathered}
$$




$$
A[x, d(t), b]=\left[\begin{array}{llcc}
g_{r 11} & g_{r 21} & 0 & 0 \\
g_{r 12} & g_{r 22} & 0 & 0 \\
g_{r 16} & g_{r 26} & g_{r 36} & 0 \\
g_{r 17} & g_{r 27} & 0 & g_{r 47}
\end{array}\right]
$$

tal que el mapa $\varphi$ es invertible para el vector de entradas $u(t)$ :

$$
u(t)=\varphi^{-1}[x, v(t), d(t), b]:=A[x, d(t), b]^{-1}\{v(t)-C[x, d(t), b]\}
$$

Por último, la dinámica cero del reactor corresponde a la dinámica de los estados $x_{I}$

$$
\dot{x}_{I I}^{*}=\left\{f_{I I}[x, d(t), b]-g_{I I}[x, d(t), b] A[x, d(t), b]^{-1} C[x, d(t), b]\right\}_{x=\left(x_{I}, x_{I I}^{*}\right)}
$$

evaluada con

$$
\begin{aligned}
& x=\left(x_{I}{ }^{\prime}, x_{I I}{ }^{\prime}\right)^{\prime}, \quad x_{I}=\left(\bar{m}_{1}, \bar{m}_{2}, \bar{T}, \bar{V}\right)^{\prime}, \quad x_{I I}^{*}=\left(p_{1}^{*}, p_{2}^{*}, i^{*}, \mu_{0}^{*}, \mu_{2}^{*}\right)^{\prime}, \\
& f_{I I}=\left(f_{r 3}, f_{r 4}, f_{r 5}, f_{r 8}, f_{r 9}\right)^{\prime}, \quad g_{I I}=\left(g_{r 3}, g_{r 4}, g_{r 5}, g_{r 8}, g_{r 9}\right)^{\prime}
\end{aligned}
$$

la cual se verificó de forma similar al caso anterior, resultando ser RE-estable.

En resumen, el problema de control con retroalimentación de estados tiene solución para el conjunto de salidas $y=\left(y_{m_{1}}, y_{m_{2}}, y_{T}, y_{V}\right)^{\prime}$, con grados relativos $k=(1,1,1,1) \mathrm{y}$ dinámica cero (5.86) RE-estable.

\subsubsection{Construcción y sintonización}

\section{A. Control con retroalimentación de mediciones}

La construcción del control con retroalimentación de mediciones viene de la aplicación directa del Teorema 2. Para el cual se construirá el exo-observador únicamente para las entradas variantes en tiempo:

$$
\begin{gathered}
x_{d}(t)=d_{1}(t)=\left(m_{1 e}, T_{1 e}, T_{2 e}, T_{s e}\right)^{\prime}, \quad d_{1}(t) \subset d(t) \\
\left(v_{1}, v_{2}, v_{3}, v_{4}\right)=(1,1,1,1)
\end{gathered}
$$

De acuerdo al Teorema 2 y al mapa $\varphi$ definido en (5.74), el controlador no lineal con retroalimentación de mediciones está dado por (las matrices $A$ y $C$, y el mapa $\phi_{\mathrm{I}}$ están definidos en (5.73), (5.72) y (5.66), respectivamente): 


$$
\begin{aligned}
u(t) & =A\left[\hat{x}, \hat{x}_{d}(t), \beta\right]^{-1}\left\{K_{c}\left(s_{c}\right)\left[\phi_{I}\left(\hat{x}, \hat{x}_{d}, \beta\right)-\phi_{I}\left(\bar{x}, \bar{x}_{d}, b\right)\right]-\hat{x}_{q}-C\left[\hat{x}, \hat{x}_{d}(t), \beta\right]\right\} \\
& :=\gamma\left(\hat{x}, \hat{x}_{q}, \hat{x}_{d}, \beta\right)
\end{aligned}
$$

y el sistema a lazo cerrado por las ecuaciones (5.56).

Para la sintonización se empleará el mismo esquema establecido en la Sección 3.7. Las ganancias de referencia para el exo-observador, el estimador, y el controlador $\left\{K_{\mathrm{u}}(1), K_{\mathrm{o}}(1), K_{\mathrm{l}}(1), K_{\mathrm{c}}(1)\right\}$ están listadas en las Tablas 3.1, 3.2. y 5.1, respectivamente. Para las cuales las frecuencias y factores de amortiguamiento de referencia se han seleccionado de forma similar a las definidas para el esquema de estimación (ver Sección 4.4). Definiendo la frecuencia natural del reactor como el inverso del tiempo nominal de residencia (ver Tabla 2.4)

$$
\omega_{r}=1 / \bar{\theta}_{r}=1 / 230 \mathrm{~min}^{-1}
$$

Las frecuencias de referencia para el exo-observador son

$$
\begin{array}{ll}
\omega_{l}^{d}=10 \omega_{r}=1 / 23 \mathrm{~min}^{-1} & \left(\text { para } m_{l e}\right) \\
\omega_{i}^{d}=7 \omega_{r}=1 / 33 \mathrm{~min}^{-1}, \quad i=2,3,4 & \left(\text { para } T_{1 e}, T_{2 e}, y T_{s e}\right)
\end{array}
$$

para el estimador

$$
\begin{array}{ll}
\omega_{1}^{o}=\omega_{2}^{o}=\omega_{r} / 2=1 / 460 \mathrm{~min}^{-1} & (\text { para } \rho y \eta) \\
\omega_{3}^{o}=\omega_{4}^{o}=2 \omega_{r}=1 / 115 \mathrm{~min}^{-1} & (\text { para } T y V) \\
\xi_{i}=0.71, & 1 \leq i \leq 4
\end{array}
$$

y para el controlador

$$
\begin{aligned}
& \omega_{1}^{c}=\omega_{2}^{c}=\omega_{r} / 2=1 / 460 \mathrm{~min}^{-1} \quad(\text { para } \rho y \eta) \\
& \omega_{3}^{c}=\omega_{4}^{c}=2 \omega_{r}=1 / 115 \mathrm{~min}^{-1} \quad(\text { para } T y \mathrm{~V})
\end{aligned}
$$

Con parámetros de ajuste de aceleración/retardo

$$
\left(s_{d}, s_{o}, s_{c}\right)=(1,7,0.7)
$$

tales que el exo-observador (tiempo de asentamiento $\tau_{a}^{d} \approx 4 \times 23 \mathrm{~min}=92 \mathrm{~min}$ ) sea más rápido que el estimador $\left(\tau_{a}^{o} \approx 4 x 65.7 \mathrm{~min}=262.8 \mathrm{~min}\right)$, y éste más rápido que el controlador $\left(\tau_{a}^{c} \approx 4 x 657 \mathrm{~min}=2628 \mathrm{~min}\right)$, de manera que la condición de convergencia (5.60) se satisface. Aquí el criterio para seleccionar los parámetros de ajuste $\left(s_{d}, s_{o}, s_{c}\right)$ (5.93) sigue siendo que el estimador sea aproximadamente 10 veces más rápido que el controlador $\left(s_{o} / s_{c}=10\right)$, y el parámetro del controlador $s_{\mathrm{c}}$ se escogió como 0.7 para que el lazo de control tuviese suficiente tiempo para converger sin sobretiros iniciales excesivos. 


\section{$B$. Control con retroalimentación de estados}

El diseño y sintonización del control con retroalimentación de estados se construye de acuerdo al Teorema 1. En este caso el controlador no lineal (5.41) está dado por (las matrices $A$ y $C$, y el mapa $\phi_{\mathrm{I}}$ están definidos en (5.84), (5.83) y (5.80), respectivamente):

$$
u(t)=A\left[x, x_{d}(t), b\right]^{-1}\left\{K_{c}\left(s_{c}\right)\left[\phi_{I}\left(x, x_{d}, b\right)-\phi_{I}\left(\bar{x}, \bar{x}_{d}, b\right)\right]-C\left[x, x_{d}(t), b\right]\right\}:=u^{o}(t)(5.94)
$$

y el sistema a lazo cerrado por las ecuaciones (5.42). Las ganancias de referencia están definidas en la Tabla 5.1, las frecuencias de referencia son las mismas ya definidas arriba (5.92), y el parámetro de ajuste se seleccionó como

$$
s_{c}=1.8
$$

Este parámetro se seleccionó más grande (i.e., más rápido con $\tau_{a}^{c o} \approx 4 \times 255 \min =1020$ min) que el parámetro de ajuste para el control con retroalimentación de mediciones $\left(\tau_{a}^{c} \approx 4 x 657 \mathrm{~min}=2628 \mathrm{~min}\right.$ ), ya que al darse por conocidos los estados no se requiere del estimador y la dinámica puede hacerse suficientemente rápida.

\subsubsection{Implementación de los controladores}

A continuación se muestran los resultados comparativos del funcionamiento de los controladores:

A. $C_{\mathrm{x}}$ con retroalimentación de estados, el cual corresponde al control exacto, sólo con errores en las condiciones iniciales, $y$

B. $C_{\mathrm{y}}$ con retroalimentación de mediciones, el cual se emplea un estimador y se consideran dos situaciones. El funcionamiento- $\mathrm{N}$ (nominal) con errores en las condiciones iniciales, $y$ el funcionamiento- $R$ (robusto) con errores en las condiciones iniciales y en los parámetros del modelo.

Los errores en los estados iniciales empleados para ambos controladores $C_{\mathrm{x}} \mathrm{y} C_{\mathrm{y}}$, así como para el estimador empleado para $C_{\mathrm{y}}$ se encuentran listados en la Tabla 5.2. Los errores en los parámetros del modelo se encuentran listados en las Tablas 2.5. Y las variaciones en tiempo de las entradas exỏgenas son las mismas empleadas para el esquema de estimación (ver Figura 4.5a). 
Tabla 5.2 Estados y salidas: (a) in iciales para la planta $x(t)$, (b) iniciales para el estimador $\hat{x}(t)$, y (c) nominales o de referencia $\bar{x}$ para regulación.

\begin{tabular}{|c|c|c|c|}
\hline \multirow{2}{*}{$x, y, z$} & \multicolumn{2}{|c|}{ Estado iniciat } & Estodo de referencia, \\
\hline & Planta $x_{0}$ & Estinador, $x_{0}$ & 0.6819 \\
\hline$x_{1}\left(m_{1}\right)$ & 0.0060 & 0.0045 & 0.4577 \\
\hline$x_{2}\left(m_{2}\right)$ & 0.4500 & 0.5000 & 0.0990 \\
\hline$x_{3}\left(p_{1}\right)$ & 0.0900 & 0.1500 & 0.2061 \\
\hline$x_{4}\left(p_{2}\right)$ & 0.1900 & 0.2500 & 0.00083 \\
\hline$x_{5}(i)$ & 0.0007 & 0.0010 & 345.1 \\
\hline$x_{6}(T)$ & 340.0 & 330.0 & 2.0 \\
\hline$x_{7}(V)$ & 2.15 & 2.35 & $1.984 \times 10^{-3}$ \\
\hline$x_{8}\left(\mu_{0}\right)$ & $2.534 \times 10^{-3}$ & $2.071 \times 10^{-3}$ & $9.933 \times 10^{7}$ \\
\hline$x_{9}\left(\mu_{2}\right)$ & $5.633 \times 10^{7}$ & $8.282 \times 10^{7}$ & 1001.76 \\
\hline$y_{p}(\rho)$ & 1000.75 & 1015.03 & 1.4229 \\
\hline$y_{\eta}(\eta)$ & 1.4206 & 1.4306 & 345.1 \\
\hline$y_{T}(T)$ & 340.0 & 330.0 & 2.0 \\
\hline$y_{V}(V)$ & 2.15 & 2.35 & 0.3606 \\
\hline$z_{s}\left(m_{s}\right)$ & 0.3333 & 0.4080 & 0.2888 \\
\hline$z_{c}(c)$ & 0.2860 & 0.3061 & 0.4439 \\
\hline$z_{p}(p)$ & 0.4274 & 0.4638 & 273,893 \\
\hline$z_{M}(M)$ & 170,000 & 200,000 & 187.3 \\
\hline$z_{R}(R)$ & 247.9 & 293.3 & \\
\hline & & & \\
\hline & & & \\
\hline
\end{tabular}

Los funcionamientos de los controladores se muestran en la Figura 5.3, y los resultados son los siguientes:

(1) Como se esperaba, con el controlador $C_{\mathrm{x}}$ hay convergencia rápida para todos los estados, salidas y entradas; los cambios son tenues y no hay sesgos finales. Este es el comportamiento máximo limite alcanzable que se puede lograr con un controlador no lineal.

(2) El funcionamiento- $N$ del controlador $C_{y}$ no tiene sesgos, y es más lento comparativamente con el controlador $C_{\mathrm{x}}$. Sin embargo, estos dos casos no son prácticos, ya que en realidad no se conocen exactamente todos los estados y el modelo del proceso no es exacto.

(3) El funcionamiento - $\mathrm{R}$ del controlador $C_{\mathrm{y}}$ es muy parecido al nominal: tiene 
aproximadamente la misma velocidad de convergencia pero con sesgos pequeños (aún aceptables).

(4) En general para todos los casos, la salida $z_{M}$ que está directamente relacionada a la dinámica cero, es más lenta que los demás estados y salidas.

(5) Los tiempos de asentamiento corresponden a los establecidos en la sintonización: aproximadamente $1000 \mathrm{~min}$ para el controlador $C_{x}$, y $2500 \mathrm{~min}$ para el controlador $C_{\mathrm{y}}$.

Comparando estos resultados con otros reportados en la literatura, para controladores diseñados para reactores continuos de copolimerización en solución, se tienen las siguiente observaciones:

(1) En Congalidis et al. (1985) se reporta un control convencional (lineal), en el cual se alcanza convergencia en tiempos muy largos, de aproximadamente 18 veces el tiempo de residencia nominal del reactor: $\tau_{a} \approx 18 \bar{\theta}_{r}$.

(2) En Padilla et al. (1995) se presentó un control no lineal con retroalimentación de estados, en el cual se logrô convergencia exponencial en un tiempo aproximado de: $\tau_{a} \approx 4 \bar{\theta}_{r}$. Siendo dicho controlador el mismo controlador $C_{\mathrm{x}}$ aquí presentado, $\mathrm{y}$ que por ello se obtiene también aqui el mismo tiempo de convergencia $\tau_{a} \approx 4 \bar{\theta}_{r}$.

(3) En Padilla y Alvarez (1997) se presenta un control con retroalimentación de mediciones (obtenido a partir de la linealización del control exacto con retroalimentación de estados), el cual muestra tiempos de asentamiento de aproximadamente: $\tau_{a} \approx 6 \bar{\theta}_{r}$. No obstante se mantiene la consideración de que al menos tres concentraciones (estados) son conocidas en linea.

(4) El control con retroalimentación de mediciones $C_{y}$ (basado en un estimador y mediciones secundarias en linea) aquí propuesto, tiene un tiempo de asentamiento de aproximadamente $\tau_{a} \approx 10 \bar{\theta}_{r}$ (ver Figura 5.3). Siendo este tiempo de asentamiento menor al de un control convencional y mayor a uno avanzado sin estimador.

Como puede verse este esquema de control tiene ventajas (como es la inferencia de las propiedades de calidad y producción a partir de cuatro mediciones en linea, instantáneas y de bajo costo) y desventajas (como es el requerir un tiempo más largo para convergencia, comparado con un control exacto). No obstante, como se mencionó en el resumen del Capítulo 4, el funcionamiento del controlador puede mejorarse: incluyendo más mediciones en línea continuas o discretas, y ajustando previamente los parámetros del modelo. Una alternativa más al esquema de control se presenta en la siguiente y última sección, considerando nuevamente el esquema de controlador-estimador. 


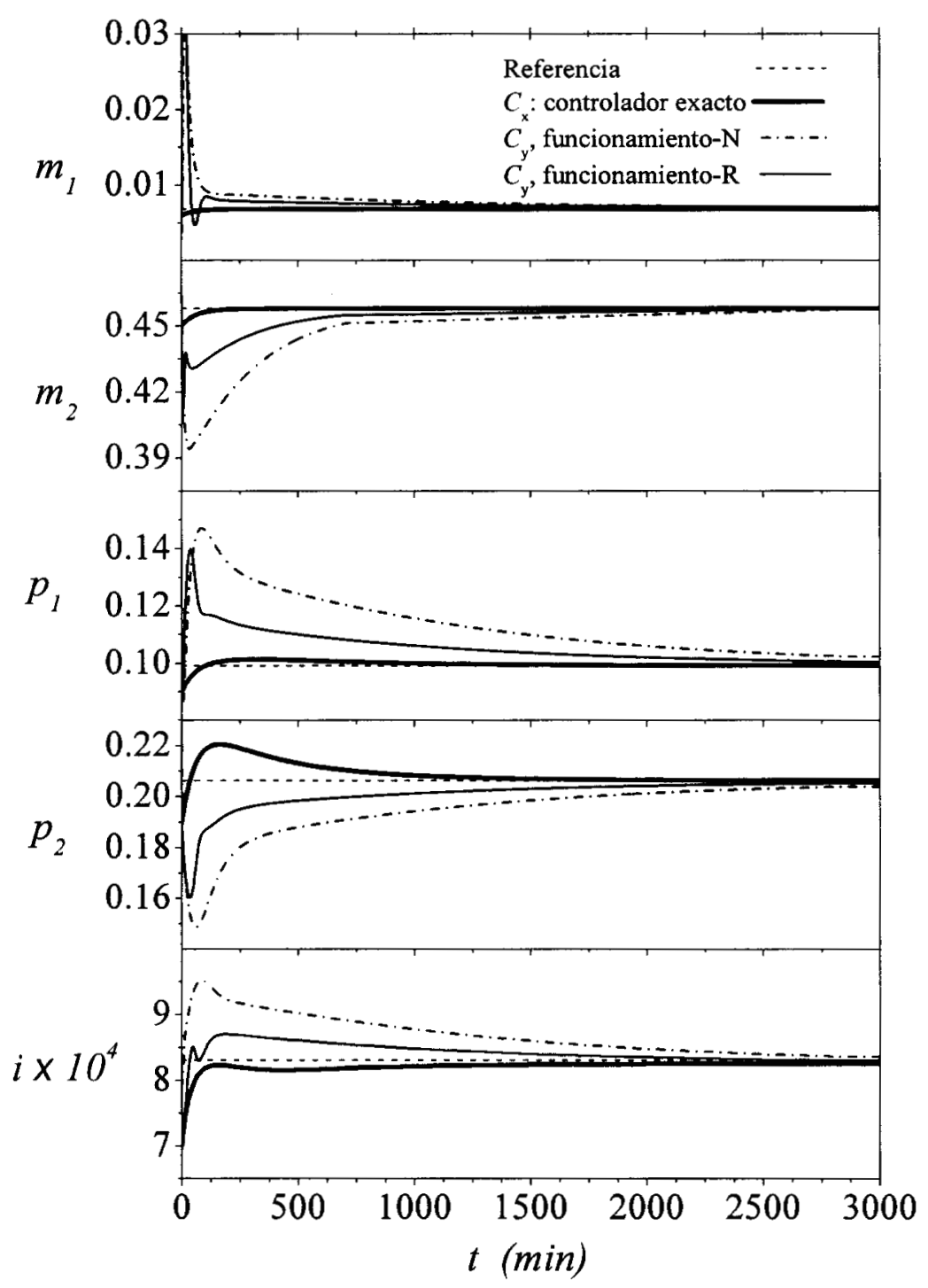

Figura 5.3 (a) Evoluciōn de los estados (x) de la planta para los controladores con retroalimentación de estados, $C_{\mathrm{x}}, \mathrm{y}$ con retroalimentación de mediciones, $C_{\mathrm{y}}$. 

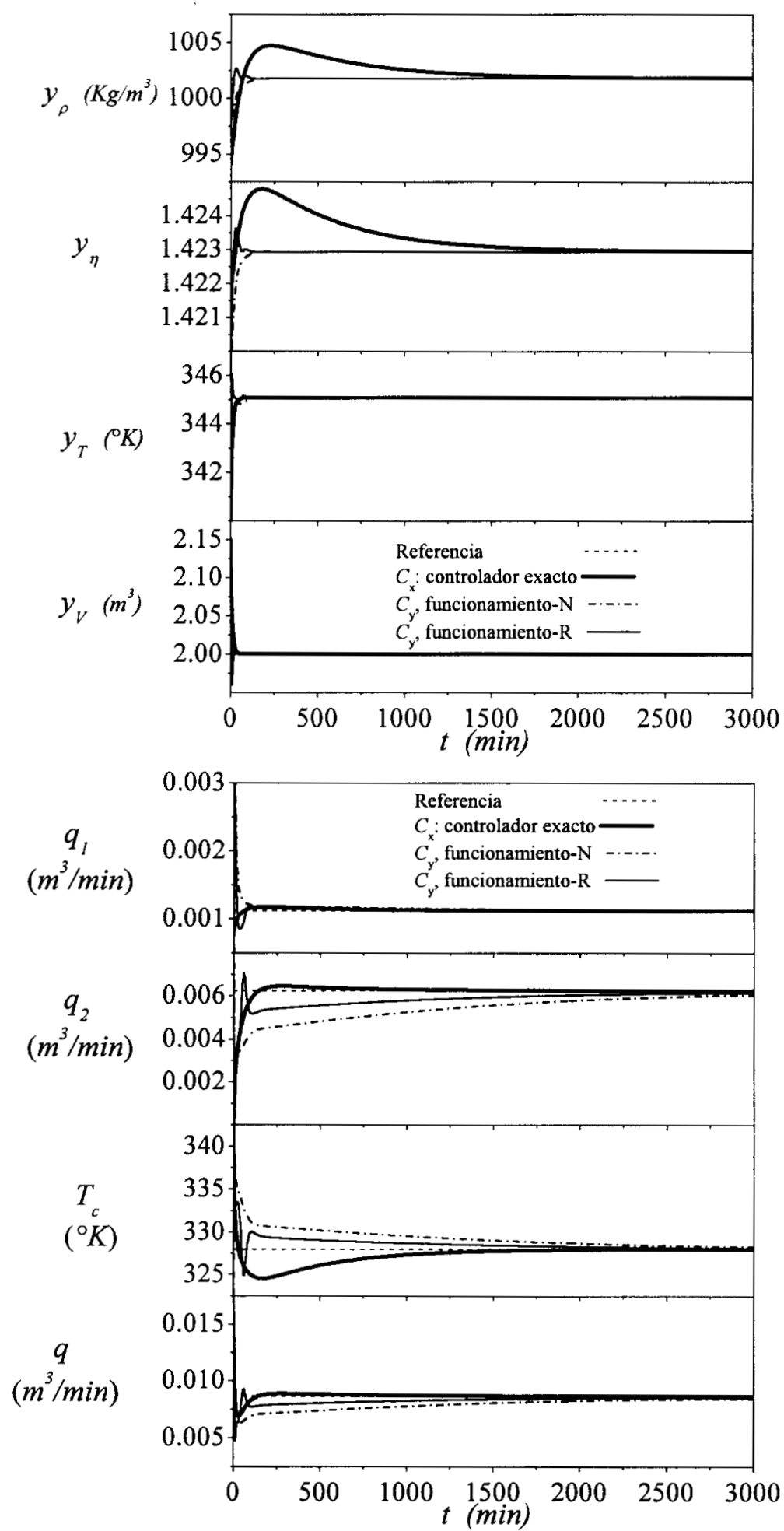

Figura 5.3 (b), (c) Evolución de las salidas (y) y de las entradas de control (u) de la planta para los controladores con retroalimentación de estados, $C_{\mathrm{x}}, \mathrm{y}$ con retroalimentación de mediciones, $C_{\mathrm{y}}$. 


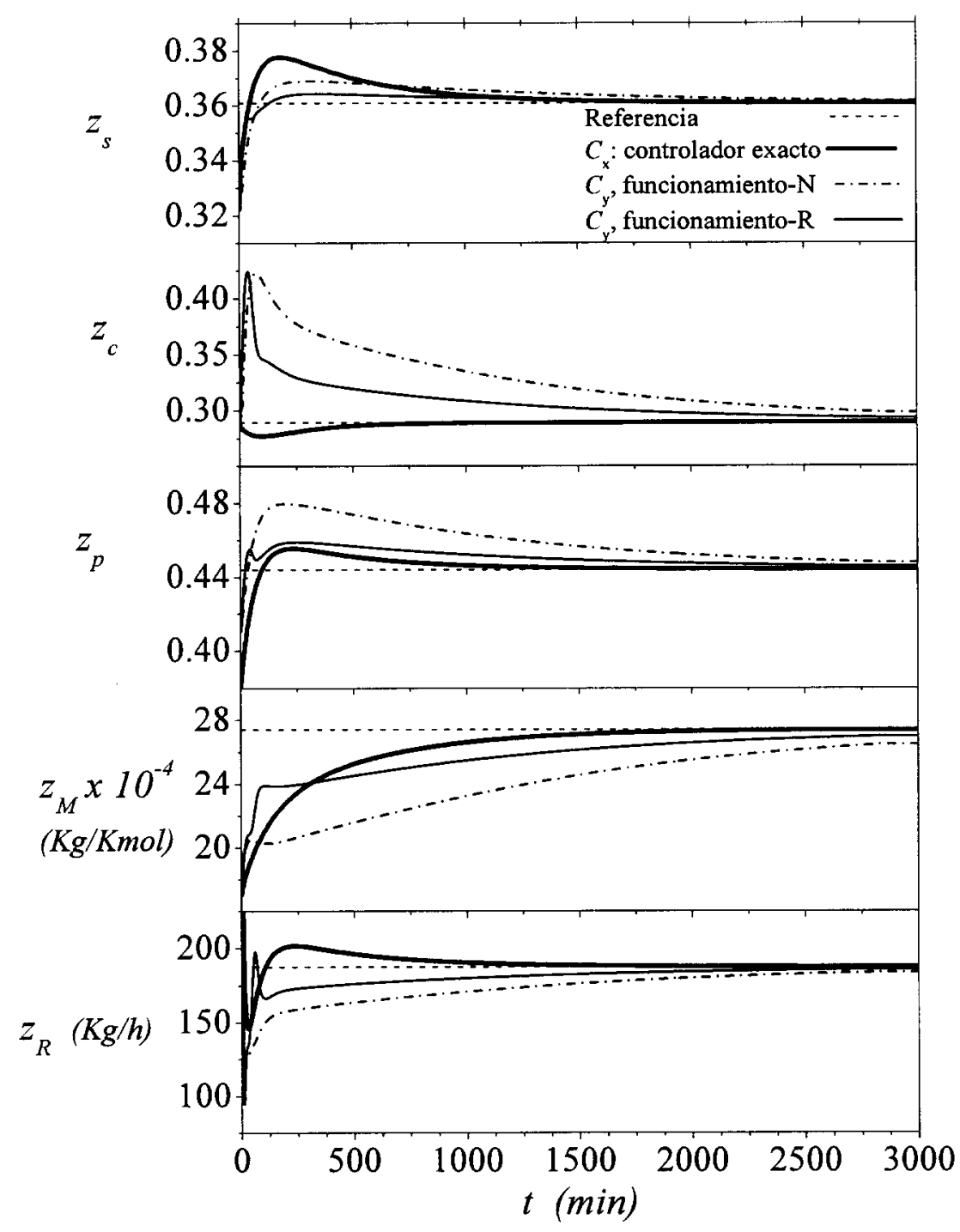

Figura 5.3 (d) Evolución de las salidas de interés $(z)$ de la planta para los controladores con retroalimentación de estados, $C_{\mathrm{x}}, \mathrm{y}$ con retroalimentación de mediciones, $C_{\mathrm{y}}$. 


\subsection{Alternativa al esque ma de control con retroalimentación de mediciones (salidas informativas diferentes a las salidas de control)}

\subsubsection{Antecedente}

De los resultados obtenidos en la sección anterior, se concluyó que el control $C_{\mathrm{y}}$ con retroalimentación de mediciones es $\mathrm{RE}$-convergente y es capaz de recuperar el control $C_{\mathrm{x}}$ con retroalimentación de estados (exacto). Sin embargo, un inconveniente en el desempeño del control $C_{y}$ es que tiene un tiempo de convergencia mayor al tiempo de convergencia del control exacto $C_{\mathbf{x}}$ (aproximadamente el doble).

Por otra parte, en el trabajo de Padilla y Alvarez (1997) se presenta un esquema de control con retroalimentación de salidas para el mismo caso de estudio (un reactor continuo de copolimerización de MMA-AV, en $\mathrm{AE}$ como solvente), donde reportan un tiempo de convergencia de aproximadamente $\tau_{a} \approx 6 \bar{\theta}_{r}$ (mismo tiempo de convergencia que el control $C_{\mathrm{x}}$ exacto). El esquema de control, presentado en la publicación mencionada, no emplea un estimador de estados; sino que el controlador es obtenido a partir de la linealización del control no lineal (exacto) con retroalimentación de estados, bajo la suposición de que tres concentraciones (monómeros remanentes, monómeros convertidos, o solvente) son conocidas. Esta última suposición es sustentada bajo la hipótesis de que, en principio el desempeño de control con retroalimentación de salidas se mantendrá si los estados son inferidos a partir de un estimador de estados. En este estudio se concluyó que las mejores estructuras de controlabilidad corresponden a:

$$
\begin{aligned}
& \text { (1) } y=\left(m_{1}, m_{2}, s, T, V\right)^{\prime}, \quad u=\left(q_{1}, q_{2}, q_{s}, T_{c}, q\right)^{\prime}, \quad k=(1,1,1,1,1)^{\prime} \\
& \text { (2) } y=\left(m_{2}, p_{1}, s, T, V\right)^{\prime}, \quad u=\left(q_{1}, q_{2}, q_{s}, T_{c}, q\right)^{\prime}, \quad k=(1,1,1,1,1)^{\prime}
\end{aligned}
$$

Tomando en cuenta los resultados obtenidos del control $C_{\mathrm{y}}$ propuesto en la sección anterior, y los obtenidos en el trabajo de Padilla y Alvarez (1997), se plantea un esquema de control modificado basado en el estimador con las siguientes caracteristicas:

(a) Las salidas $y_{0}$ informativas (o mediciones indirectas), a partir de las cuales se construirá el estimador, son diferentes a las salidas $y_{c}$ de regulación (o salidas de control), a partir de las cuales se construirá el control con retroalimentación de los estados inferidos. Para el caso del reactor continuo de copolimerización, estas salidas serán:

$$
y_{o}=\left(y_{p}, y_{\eta}, y_{T}, y_{V}\right)^{\prime}, \quad y_{c}=\left(y_{m_{1}}, y_{m_{2}}, y_{T}, y_{V}\right)^{\prime}
$$


(b) Se empleará un doble integrador, uno para el estimador y otro para el controlador.

A continuación se presenta la construcción, sintonización e implementación de este último esquema propuesto; quedando como trabajo futuro la justificación teórica del criterio de convergencia.

\subsubsection{Construcción y sintonización del controlador}

Sean las salidas medidas

$$
y_{o}=\left(y_{1}^{o}, \ldots, y_{m}^{o}\right)^{\prime}:=h_{o}(x, b)
$$

para las cuales la planta (5.1) es RE-estimable (de acuerdo a la Definición 2 Capitulo 3), con estructura de estimabilidad $S$,

$$
\begin{array}{rlrl}
S=\left\{k_{o}, x_{I}^{o}\right\}, & k_{o}=\left(\kappa_{1}^{o}, \ldots, \kappa_{m}^{o}\right)^{\prime}, & \kappa_{o}=\kappa_{1}^{o}+\ldots+\kappa_{m}^{o} \\
x_{I}^{o}=\left[\phi_{I}^{o}\left(x, x_{d}, b\right)\right]^{-1}, & \operatorname{dim}\left(x_{I}^{o}\right)=\kappa_{o}
\end{array}
$$

y con estados no observables $x_{I I}^{o}$

$$
x_{I I}^{o}=\phi_{I I}^{o}(x), \quad x=I_{p}\left(x_{I}^{o \prime}, x_{I I}^{o \prime}\right)^{\prime}, \quad \operatorname{dim}\left(x_{I I}^{o}\right)=n-\kappa_{o}
$$

cuya dinámica es RE-estable. Entonces el estimador-PI a lazo abierto está dado por (ver Teorema 3 - Capitulo 3, con dos modificaciones: el vector de entradas exógenas $x_{u}$ del estimador ahora corresponde al vector $x_{d}$ con matrices $K_{d}, \Gamma_{d} \mathrm{y} \Delta_{d}$ en vez de $K_{u}$, $\Gamma_{u}$ y $\Delta_{u}$, y la acción integral se incluye como un estado adicional, $\left.x_{q}\right)$ :

$$
\begin{aligned}
& \dot{\hat{x}}_{d}=\Gamma_{d} \hat{x}_{d}+K_{d}\left(s_{d}\right)\left(d-\Delta_{d} \hat{x}_{d}\right), \quad \hat{d}=\Delta_{d} \hat{x}_{d} \\
& \dot{\hat{x}}_{q}=K_{I}^{o}\left(s_{o}\right)\left[y_{o}-h_{o}(\hat{x}, \beta)\right] \\
& \dot{\hat{x}}=f\left[\hat{x}, u(t), \Delta_{d} \hat{x}_{d}, \beta\right]+G\left(\hat{x}, \hat{x}_{d}, \beta\right)\left[y_{o}-h_{o}(\hat{x}, \beta)\right]+H\left(\hat{x}, \hat{x}_{d}, \beta\right) \hat{x}_{q} \\
& \hat{y}_{o}=h_{o}(\hat{x}, \beta), \quad \hat{z}=h_{z}(\hat{x}, \beta) \\
& G\left(\hat{x}, \hat{x}_{d}, \beta\right)=\left[I_{11}^{\prime}, I_{12}^{\prime}\right]\left[\phi_{I x_{l}}^{o}\left(\hat{x}, \hat{x}_{d}, \beta\right)\right]^{-1} K_{o}\left(s_{o}\right) ; \\
& H\left(\hat{x}, \hat{x}_{d}, \beta\right)=\left[I_{11}{ }^{\prime}, I_{12}{ }^{\prime}\right]\left[\phi_{I x_{l}}^{o}\left(\hat{x}, \hat{x}_{d}, \beta\right)\right]^{-1} \Pi_{o}
\end{aligned}
$$

En el cual $\hat{x}_{q}$ corresponde a la estimación de la parte lenta de la perturbación persistente de la dinámica observable, debido principalmente a errores en los parámetros del modelo; y converge asintóticamente a (ecuación 3.60 en coordenadas originales):

$$
\hat{x}_{q} \rightarrow x_{q}=\varphi_{o}\left[x, u(t), x_{d}(t), b\right]-\varphi_{o}\left[\hat{x}, u(t), \hat{x}_{d}(t), \beta\right]
$$


Por otra parte, si la planta (5.1) es sujeta al controlador con retroalimentación de estados exacto (5.41) (sin estimador), construido a partir de las salidas

$$
y_{c}=\left(y_{1}^{c}, \ldots, y_{m}^{c}\right)^{\prime}:=h_{c}(x, b)
$$

con grados relativos iguales a los indices de observabilidad

$$
k_{c}=k_{o} \quad \Rightarrow \quad\left(\kappa_{1}^{o}, \ldots, \kappa_{m}^{o}\right)^{\prime}=\left(\kappa_{1}^{o}, \ldots, \kappa_{m}^{o}\right)^{\prime}
$$

$\mathrm{y}$ con estados no controlables $x_{I I}^{c}$ definidos iguales a los no observables $x_{I I}^{o}(5.98)$

$$
x_{I I}^{c}=x_{I I}^{o}, \quad \operatorname{dim}\left(x_{I I}^{c}\right)=n-\kappa_{c}
$$

cuya dinámica (5.98) es RE-estable. Entonces el controlador exacto con retroalimentación de estados está dado por

$$
u^{o}(t)=\varphi_{c}^{-1}\left\{x, \bar{v}+K_{c}\left[\phi_{I}^{c}\left(x, x_{d}, b\right)-\phi_{l}^{c}\left(\bar{x}, \bar{x}_{d}, b\right)\right], x_{d}(t), b\right\}
$$

No obstante si existen errores de modelado, entonces el controlador $u(t)$

$$
u(t)=\varphi_{c}^{-1}\left\{x, \overline{\mathrm{v}}+K_{c}\left[\phi_{I}^{c}\left(x, x_{d}, \beta\right)-\phi_{I}^{c}\left(\bar{x}, \bar{x}_{d}, b\right)\right], x_{d}(t), \beta\right\}
$$

tendrá un sesgo $x_{c}$ persistente, por la diferencia entre estas dos ecuaciones:

$$
\begin{aligned}
x_{c}(t)= & \varphi_{c}^{-1}\left\{x, \overline{\mathrm{v}}+K_{c}\left[\phi_{I}^{c}\left(x, x_{d}, b\right)-\phi_{I}^{c}\left(\bar{x}, \bar{x}_{d}, b\right)\right], x_{d}(t), b\right\}- \\
& \varphi_{c}^{-1}\left\{x, \overline{\mathrm{v}}+K_{c}\left[\phi_{I}^{c}\left(x, x_{d}, \beta\right)-\phi_{I}^{c}\left(\bar{x}, \bar{x}_{d}, b\right)\right], x_{d}(t), \beta\right\}
\end{aligned}
$$

el cual puede estimarse a partir del integrador

$$
\dot{\hat{x}}_{c}=\left[K_{l}^{c}\left(s_{c}\right)\right]\left[h_{c}(x, \beta)-\bar{y}\right], \quad y_{c}=h_{c}(x, \beta)
$$

Implicando que el controlador [con errores de modelado (5.105), y corregido con $x_{\mathrm{c}}$ (5.107)] está dado por

$$
u(t)=\varphi_{c}^{-1}\left\{x, \bar{v}+K_{c}\left[\phi_{I}^{c}\left(x, x_{d}, \beta\right)-\phi_{I}^{c}\left(\bar{x}, \bar{x}_{d}, b\right)\right]-\hat{x}_{c}, x_{d}(t), \beta\right\}:=\sigma\left(x, x_{d}, x_{c}, \beta\right)
$$

para el cual $\hat{x}_{c} \rightarrow x_{c}$, y $u(t) \rightarrow u^{0}(t)$.

Por último, consideremos la combinación de este controlador (5.108) (reconstructor del control con retroalimentación de estados) con el estimador (5.99) a lazo abierto, generando un controlador "candidato" forzado tanto por las salidas informativas $y_{\circ}$ como por las salidas de control $y_{\mathrm{c}}$ :

$$
\begin{aligned}
& \dot{\hat{x}}_{d}=\Gamma_{d} \hat{x}_{d}+K_{d}\left(s_{d}\right)\left(d-\Delta_{d} \hat{x}_{d}\right), \quad \hat{d}=\Delta_{d} \hat{x}_{d} \\
& \dot{\hat{x}}_{q}=K_{I}^{o}\left(s_{o}\right)\left[y_{o}-h_{o}(\hat{x}, \beta)\right] \\
& \dot{\hat{x}}_{c}=\left[K_{I}^{c}\left(s_{c}\right)\right]\left[h_{c}(\hat{x}, \beta)-\bar{y}\right] \\
& \dot{\hat{x}}=f\left[\hat{x}, \sigma\left(\hat{x}, \hat{x}_{d}, \hat{x}_{c}, \beta\right), \Delta_{d} \hat{x}_{d}, \beta\right]+G\left(\hat{x}, \hat{x}_{d}, \beta\right)\left[y_{o}-h_{o}(\hat{x}, \beta)\right]+H\left(\hat{x}, \hat{x}_{d}, \beta\right) \hat{x}_{q} \\
& \hat{y}_{o}=h_{o}(\hat{x}, \beta), \quad \hat{y}_{c}=h_{c}(\hat{x}, \beta), \quad \hat{z}=h_{z}(\hat{x}, \beta), \quad u(t)=\sigma\left(\hat{x}, \hat{x}_{d}, \hat{x}_{c}, \beta\right)
\end{aligned}
$$


Tal que la planta a lazo cerrado está dada por

$$
\begin{aligned}
& \dot{\hat{x}}_{d}=\Gamma_{d} \hat{x}_{d}+K_{d}\left(s_{d}\right)\left(d-\Delta_{d} \hat{x}_{d}\right), \quad \hat{d}=\Delta_{d} \hat{x}_{d} \\
& \dot{\hat{x}}_{q}=K_{I}^{o}\left(s_{o}\right)\left[y_{o}-h_{o}(\hat{x}, \beta)\right] \\
& \dot{\hat{x}}_{c}=\left[K_{I}^{c}\left(s_{c}\right)\right]\left[h_{c}(\hat{x}, \beta)-\bar{y}\right] \\
& \dot{\hat{x}}=f\left[\hat{x}, \sigma\left(\hat{x}, \hat{x}_{d}, \hat{x}_{c}, \beta\right), \Delta_{d} \hat{x}_{d}, \beta\right]+G\left(\hat{x}, \hat{x}_{d}, \beta\right)\left[y_{o}-h_{o}(\hat{x}, \beta)\right]+H\left(\hat{x}, \hat{x}_{d}, \beta\right) \hat{x}_{q} \\
& \dot{x}=f\left[x, \sigma\left(\hat{x}, \hat{x}_{q}, \hat{x}_{d}, \beta\right), d(t), b\right] \\
& \hat{y}_{o}=h_{o}(\hat{x}, \beta), \quad \hat{y}_{c}=h_{c}(\hat{x}, \beta), \quad \hat{z}=h_{z}(\hat{x}, \beta), \quad u(t)=\sigma\left(\hat{x}, \hat{x}_{d}, \hat{x}_{c}, \beta\right)
\end{aligned}
$$

con ganancias parametrizadas

$$
\begin{aligned}
& K_{d}\left(s_{d}\right)=d b\left[\left(s_{d} k_{11}^{d}, \ldots, s_{d}^{v_{1}} k_{v_{1} 1}^{d}\right)^{\prime}, \ldots,\left(s_{d} k_{1 p}^{d}, \ldots, s_{d}^{v_{p}} k_{v_{p} p}^{d}\right)^{\prime}\right], \quad s_{d}>0 \\
& K_{o}\left(s_{o}\right)=d b\left[\left(s_{o} k_{11}^{o}, \ldots, s_{o}^{\mathrm{k}_{1}} k_{\kappa_{1} 1}^{o}\right)^{\prime}, \ldots,\left(s_{o} k_{1 m}^{o}, \ldots, s_{o}^{\kappa_{m}} k_{\kappa_{m} m}^{o}\right)^{\prime}\right], \quad s_{o}>0 \\
& K_{c}\left(s_{c}\right)=d b\left[\left(s_{c}^{\mathrm{K}_{1}^{c}} k_{11}^{c}, \ldots, s_{c} k_{1 \kappa_{1}^{c}}^{c}\right)^{\prime}, \ldots,\left(s_{c}^{\kappa_{m}^{c}} k_{m 1}^{c}, \ldots, s_{c} k_{m \kappa_{m}^{c}}^{c}\right)^{\prime}\right], \quad s_{c}>0 \\
& K_{I}^{o}\left(s_{o}\right)=\operatorname{diag}\left[s_{o}^{\mathrm{K}_{1}+1} k_{1}^{I}, \ldots, s_{o}^{\mathrm{K}_{m}+1} k_{\kappa_{m}}^{I}\right], \quad K_{I}^{c}\left(s_{c}\right)=\operatorname{diag}\left[s_{c}^{\kappa_{1}^{c}+1} k_{1}^{I}, \ldots, s_{c}^{\kappa_{m}^{c}+1} k_{\kappa_{m}}^{I}\right]
\end{aligned}
$$

y entradas de referencia, $\left\{K_{d}(1), K_{o}(1), K_{l}^{o}(1), K_{c}(1), K_{l}^{o}(1)\right\}$, que hacen estable las dinámicas LNPA del error

$$
\begin{array}{lll}
\mu_{i}^{\left(v_{i}\right)}+k_{1 i}^{d} \mu_{i}^{\left(v_{i}-1\right)}+\ldots+k_{v_{i} i}^{d} \mu_{i}=0, & 1 \leq i \leq p ; & \mu_{i}=\hat{d}_{i}-d_{i} \\
v_{i}^{\left(\kappa_{i}+1\right)}+k_{1 i}^{o} v_{i}^{\left(\kappa_{i}\right)}+\ldots+k_{\kappa_{i} i}^{o} \dot{v}_{i}+k_{i}^{I} v_{i}=0, & 1 \leq i \leq m ; & v_{i}=\hat{y}_{i}^{o}-y_{i}^{o} \\
\eta_{i}^{\left(\kappa_{i}^{c}+1\right)}-k_{i \kappa_{i}^{j}}^{c} \eta_{i}^{\left(\kappa_{i}^{c}\right)}-\ldots-k_{i 1}^{c} \dot{\eta}_{i}-k_{i}^{I} \eta_{i}=0, & 1 \leq i \leq m ; & \eta_{i}=\hat{y}_{i}^{c}-\bar{y}_{i}^{c}
\end{array}
$$

Observación 5.6. Como puede observarse en las Figuras 5.1 y 5.4, la diferencia entre los dos controladores consiste el tipo de salidas empleadas. Para el controlador basado en un estimador (presentado en secciones anteriores), llamado $C_{y}$, tiene salidas informativas iguales a las salidas de control $(y)$; mientras que este "nuevo controlador propuesto" (5.108), que llamaremos $C_{\mathrm{y} 2}$, se basa en medidas informativas o medidas $\left(y_{0}\right)$ para el estimador y en medidas de control $\left(y_{\mathrm{c}}\right)$ para el controlador. 


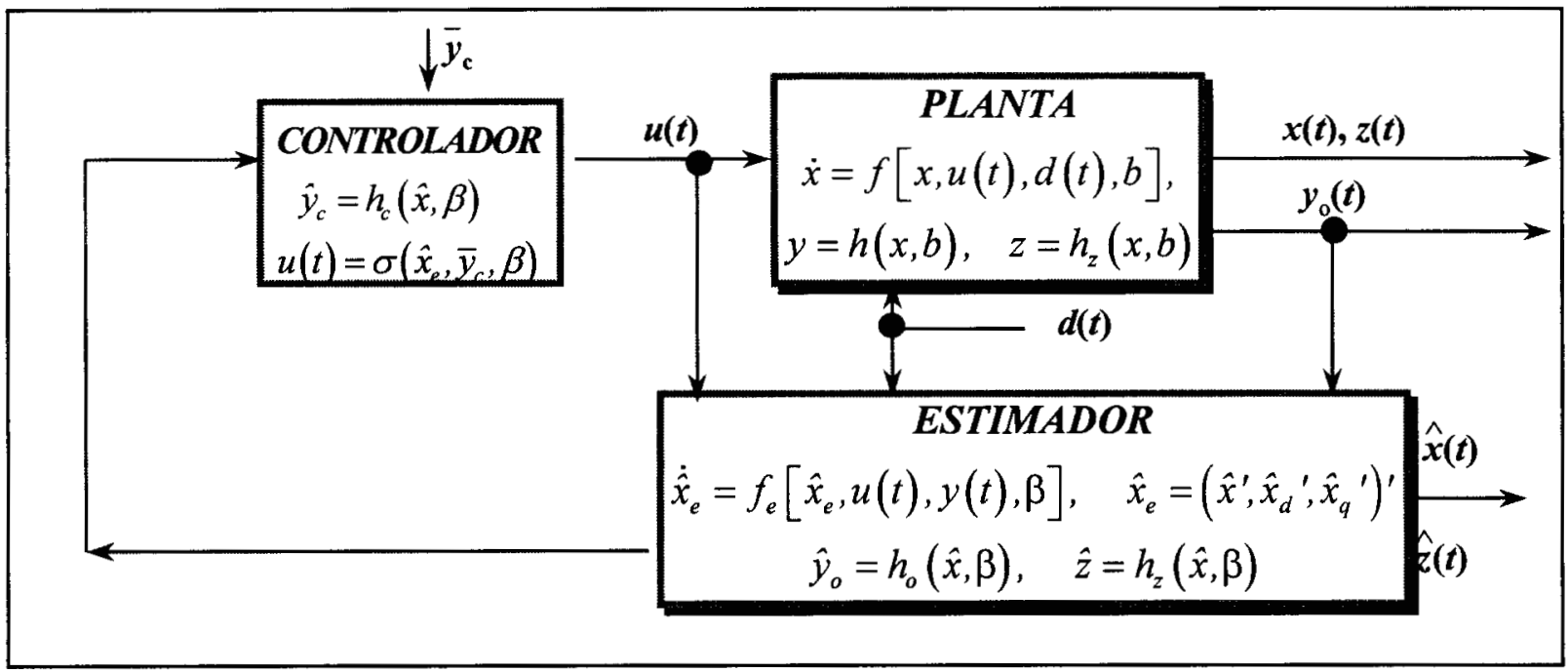

Figura 5.4 Diagrama de bloques del control con retroalimentación de mediciones, basado en salidas informativas $y_{\mathrm{o}}$ diferentes a las salidas de control $y_{\mathrm{c}}$.

5.5.3 Implementación del controlador para el reactor continuo de copolimerización en solución

La implementación de este último esquema de control con retroalimentación de mediciones $C_{\mathrm{y} 2}$, se emplearon las siguientes salidas (definidas y justificadas en la sección 5.5.1):

$$
y_{o}=\left(y_{\rho}, y_{\eta}, y_{T}, y_{V}\right)^{\prime}, \quad y_{c}=\left(y_{m_{1}}, y_{m_{2}}, y_{T}, y_{V}\right)^{\prime}
$$

Para la sintonización se emplean las mismas frecuencias $y$ factores de amortiguamiento de referencia establecidas para los controladores $C_{\mathrm{x}}$ y $C_{\mathrm{y}}$ (ver sección 5.4.2); y los parámetros de ajuste del exo-observador, estimador, y controlador se seleccionaron como

$$
\left(s_{d}, s_{o}, s_{c}\right)=(1,7,1.35)
$$

De tal forma que el exo-observador $y$ el estimador a lazo cerrado sean suficientemente rápidos, y el controlador tenga el tiempo de convergencia reportado por Padilla y Alvarez (1997) (aproximadamente $6 \bar{\theta}_{r} \approx 1380 \mathrm{~min}$ ). 
Los errores en las condiciones iniciales se encuentran listadas en la Tabla 5.2, y los errores en los parámetros del modelo están en la Tabla 2.5.

$\mathrm{E} 1$ funcionamiento- $\mathrm{N}$ (nominal) y el funcionamiento-R (robusto) del controlador $C_{\mathrm{y} 2}$ (con retroalimentación de salidas informativas y de control) se muestran en la Figura 5.5, asi como el control exacto $C_{\mathrm{x}}$ (con retroalimentación de estados) con fines de comparación. Los resultados son los siguientes:

(1) El controlador $C_{\mathrm{y} 2}$ (nominal y robusto) convergen sin sesgos, con una velocidad de convergencia mayor a la del controlador $C_{\mathrm{y}}$ (ver Figura 5.3).

(2) El funcionamiento- $\mathrm{R}$ es ligeramente más lento que el funcionamiento- $\mathrm{N}$, y tiene un comportamiento más oscilatorio debido al doble integrador.

(3) Los estados y las salidas $z$ más lentos son aquellos ligados a la dinámica cero, principalmente: $p_{1}, p_{2}, i, z_{\mathrm{c}}, \mathrm{y} z_{\mathrm{M}}$.

(4) El desempeño de las entradas de control (u) es ligeramente oscilatorio, debido al integrador del lazo de control, logrando una convergencia râpida (en aproximadamente $1000 \mathrm{~min}$ que equivale a $4 \bar{\theta}_{r}$ ).

(5) La convergencia del controlador $C_{\mathrm{y} 2}$ se logra en aproximadamente $1400 \mathrm{~min}$ (tiempo establecido en la sintonización), equivalente a $6 \bar{\theta}_{r}$. Comparado con el controlador exacto $C_{\mathrm{x}}$ que converge en aproximadamente $4 \bar{\theta}_{r}$.

Resumiendo, el control $C_{\mathrm{y} 2}$ (con retroalimentación de salidas informativas y de control) presenta el mejor desempeño aceptable con tiempo de convergencia mayor al control $C_{\mathrm{y}}$ (con retroalimentación de mediciones, i.e. salidas informativas = salidas de control) y menor al control exacto $C_{\mathrm{x}}$. 


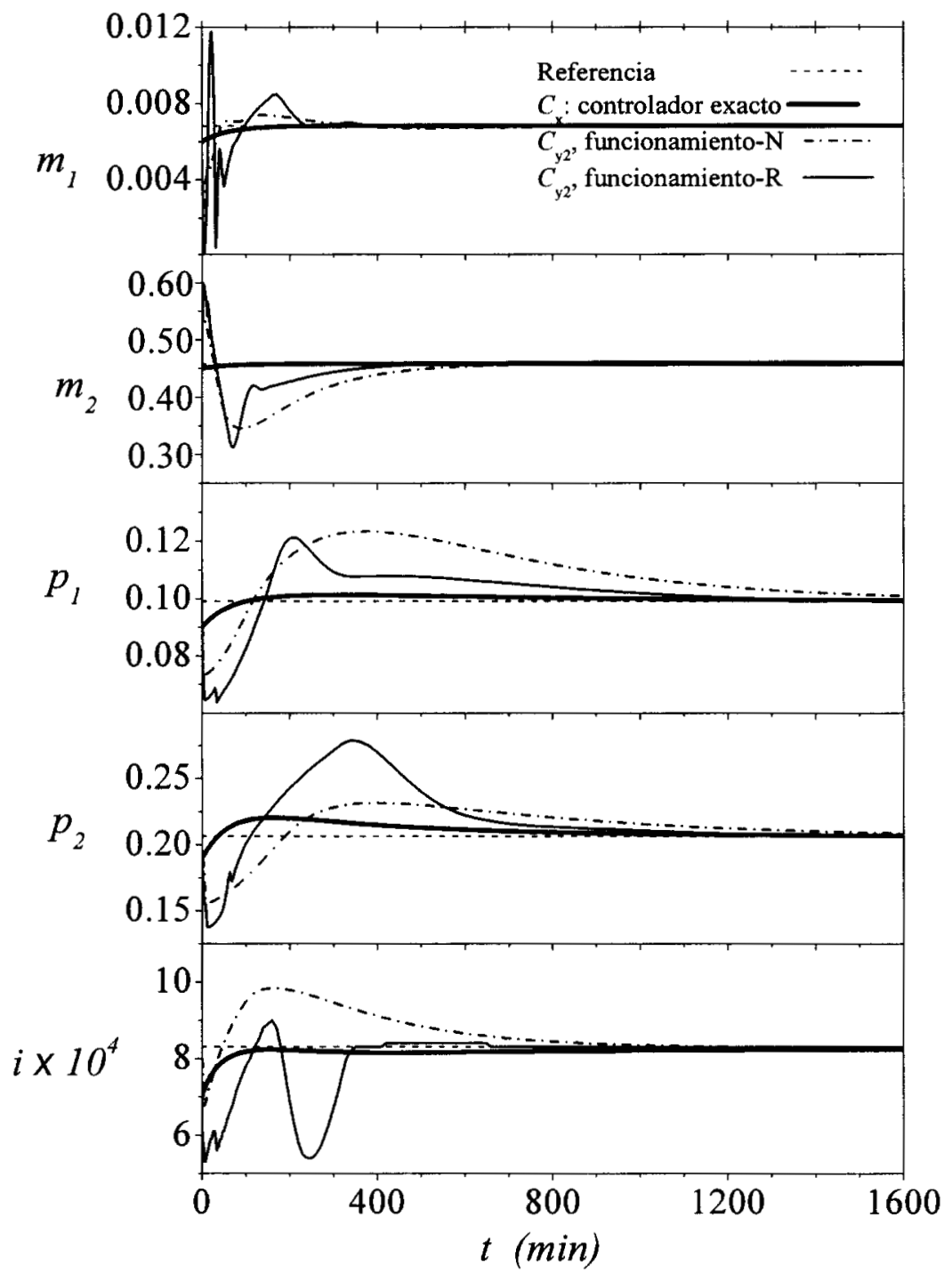

Figura 5.5 (a) Evolución de los estados $(x)$ de la planta para los controladores con retroalimentación de estados, $C_{\mathrm{x}}$, y con retroalimentación de salidas informativas $\mathrm{y}$ de control, $C_{\mathrm{y} 2}$. 

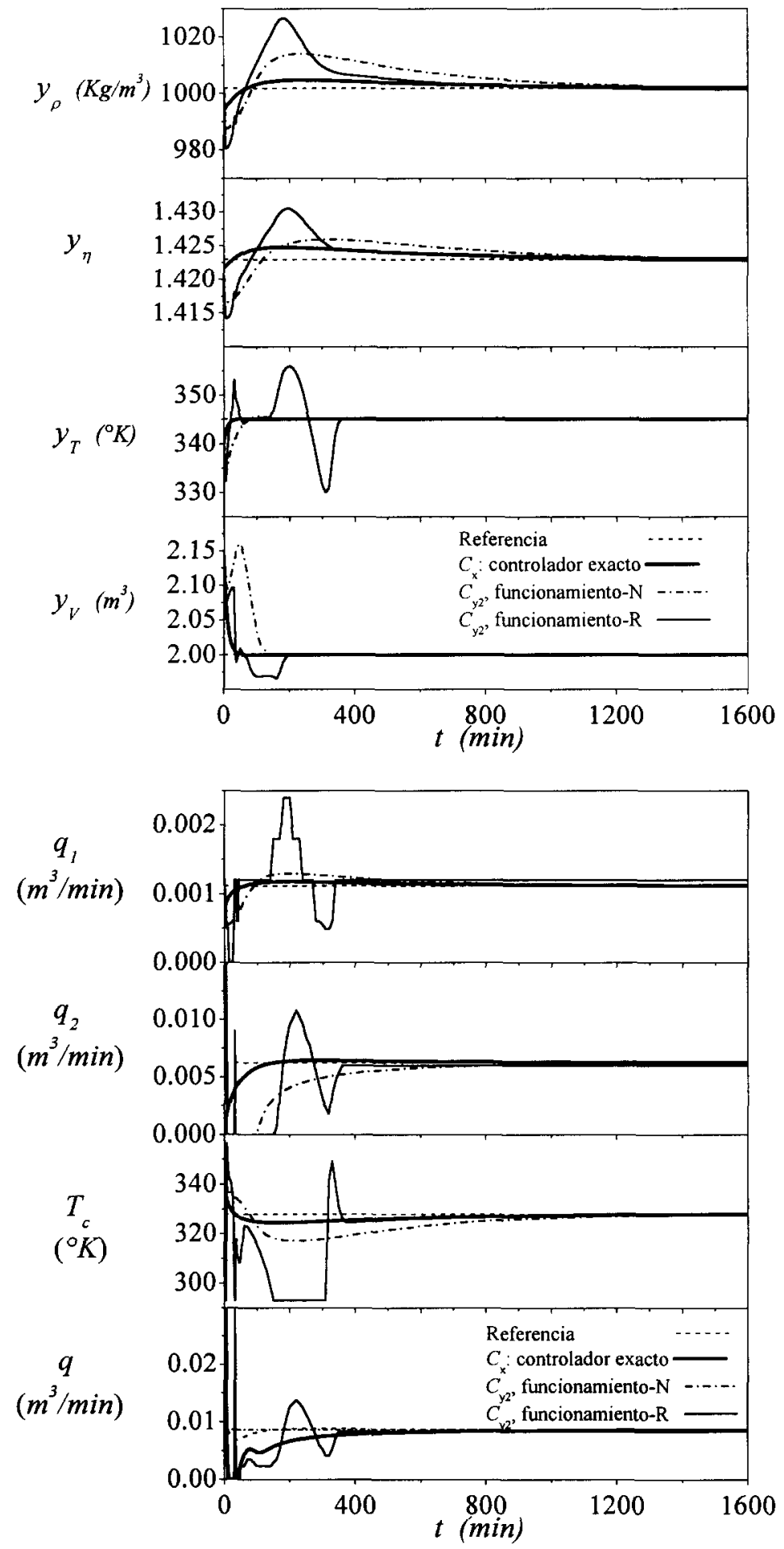

Figura 5.5 (b), (c) Evolución de las salidas (y) y de las entradas de control $(u)$ de la planta para los controladores con retroalimentación de estados, $C_{\mathrm{x}}, \mathrm{y}$ con retroalimentación de mediciones con retroalimentación de salidas informativas y de control, $C_{\mathrm{y} 2}$. 


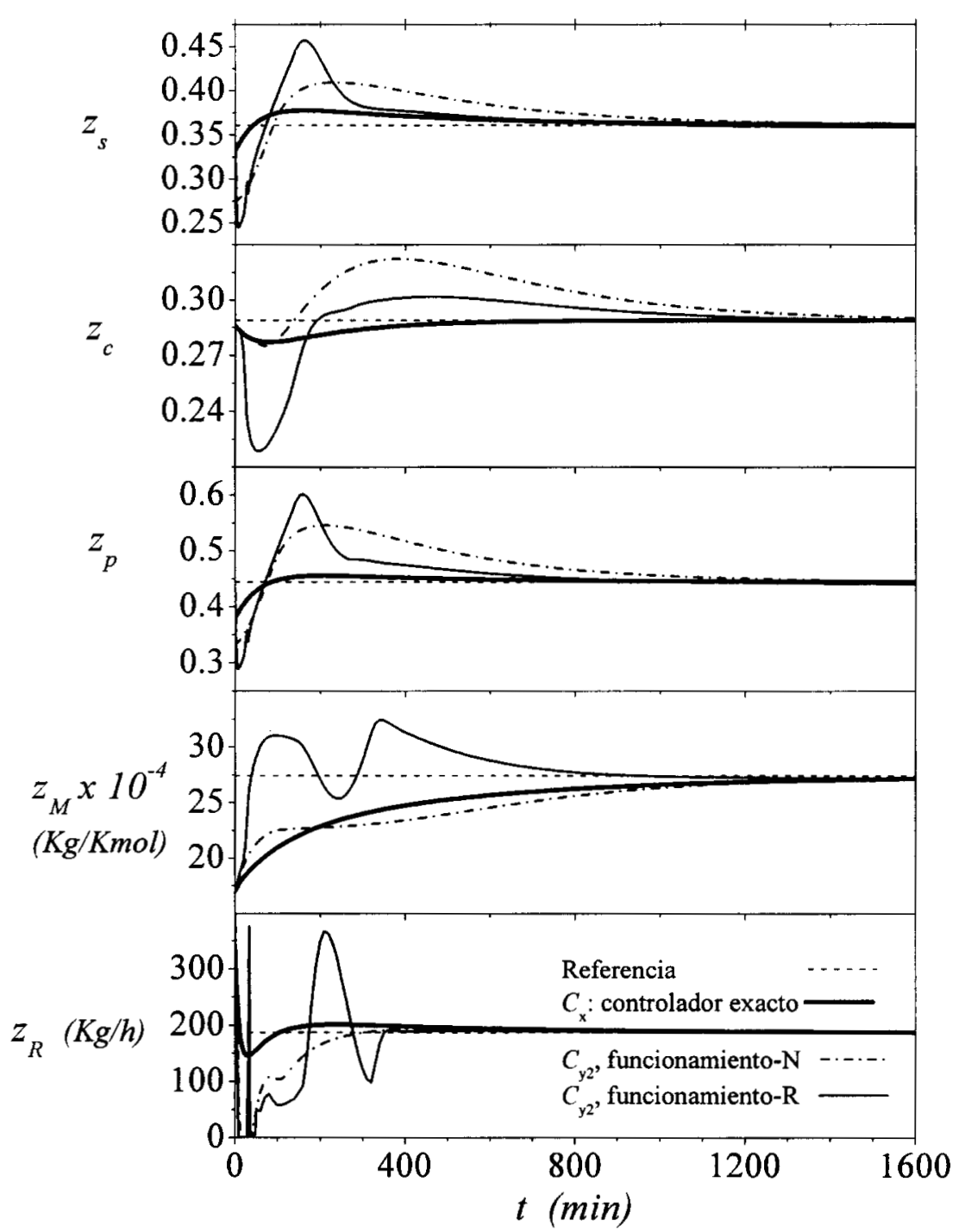

Figura 5.5 (d) Evolución de las salidas de interés $(z)$ de la planta para los controladores con retroalimentación de estados, $C_{\mathrm{x}}$, y con retroalimentación de mediciones (con medidas informativas, $y_{\mathrm{o}}$, diferentes a las salidas reguladas, $y_{\mathrm{c}}$ ), $C_{\mathrm{y}}{ }^{*}$. 


\subsection{Resumen}

En este capitulo se presentó una metodologia de control no lineal con retroalimentación de mediciones. De forma similar al esquema de estimación a lazo abierto, el esquema de control propuesto tiene las siguientes caracteristicas: (a) un procedimiento de construcción sistemático, (b) una técnica de sintonización simple, sistemática, y con interpretación física, (c) un criterio de convergencia, y (d) la estructura de estimabilidad/controlabilidad es un grado de libertad en el diseño.

Dos esquemas de control fueron formulados: un primer esquema donde la estructura de estimabilidad es igual a la estructura de controlabilidad (i.e. salidas medidas iguales a las salidas de control, grados de observabilidad iguales a los grados relativos, y estados no observables iguales a los estados no controlables), y se emplea un solo integrador para compensar errores de modelado; y un segundo esquema donde las estructuras de estimabilidad y de controlabilidad son diferentes (i.e. salidas medidas o informativas son diferentes a las salidas de control, pero con grados de observabilidad iguales a los grados relativos, y estados no observables iguales a los estados no controlables), y empleando un doble integrador.

El desempeño de ambos controladores, con retroalimentación de mediciones, para el reactor de copolimerización mostraron la factibilidad de regular las variables de seguridad, calidad, y velocidad de producción mediante mediciones secundarias en línea de temperaturas, flujos, volumen, densidad e indice de refracción. La principal diferencia entre los dos esquemas de control implementados fue la velocidad de convergencia:

(a) El control exacto (i.e. control con retroalimentación de estados, el cual fue implementado sólo para fines de comparación) converge en aproximadamente cuatro tiempos de residencia del reactor $\left(\tau_{a} \approx 4 \bar{\theta}_{r}\right)$.

(b) El control con retroalimentación de mediciones considerando la estructura de estimabilidad igual a la estructura de controlabilidad converge en $\tau_{a} \approx 10 \bar{\theta}_{r}$.

(c) El control con retroalimentación de mediciones considerando la estructura de estimabilidad diferente a la estructura de controlabilidad converge en $\tau_{a} \approx 6 \bar{\theta}_{r}$.

Con los resultados aqui obtenidos, se observa nuevamente que la mezcla de estrategias de la teoria de control geométrico no lineal con las de ingenieria de reactores de polimerización permite que la resolución del problema sea manejable y estructural. Sin embargo en el tema control con retroalimentación de mediciones, aún hay diversos aspectos por estudiar: (i) el criterio de convergencia robusto (Alvarez y López, 2000; Alvarez et al., 2000), (ii) el caso no local ; (iii) la justificación de convergencia del segundo esquema de control aquí propuesto, entre otros. 


\section{Capítulo 6}

\section{CONCLUSIONES}

En este último capítulo se discuten y resumen los resultados y contribuciones de esta tesis. Además, se dan algunas recomendaciones y trabajo futuro de investigación. 


\subsection{Conclusiones generales y aportaciones}

En esta tesis se abordaron los problemas de estimación y control no lineal en reactores continuos de copolimerización en solución. Se establecieron metodologias para la resolución de estos problemas, mostrando la factibilidad de inferir y regular las propiedades del copolimero que determinan la calidad (i.e. conversión, composición, y peso molecular promedio), la seguridad (i.e. fracción de sólidos y temperatura de operación), y la velocidad de producción; a partir de mediciones en línea (continuas, instantáneas y de bajo costo) de: densidad, indice de refracción, volumen, temperaturas, y flujos de alimentación y de descarga.

Las aportaciones principales de esta tesis son: (i) el diseño de estrategias de estimación y de control no lineal altamente sistematizadas, y (ii) su aplicación a reactores de copolimerización en solución dentro un marco metodológico.

En ingenieria de procesos, un diseño de estimación/control geométrico no lineal debe llevarse a cabo dentro de un marco metodológico, donde la etapa de la diseño son interpretadas y apoyadas con el conocimiento físico, el modelado, los métodos, y los criterios de ingenieria. Y por otra parte, la teoría del control geométrico no lineal proporciona el marco conceptual, el soporte teórico, y un procedimiento sistemático de diseño. De tal forma que la resolubilidad y las condiciones de convergencia se proporcionan con significado físico, y se contribuye con una alta sistematización, identificando e interpretando las capacidades del estimador/controlador inherentes, asi como las limitaciones asociadas a la fisica e ingenieria del proceso en particular. De aqui que la aplicabilidad y la facilidad de manipulación del estimador y de los controladores propuestos, reside en la combinación de la metodologia de la teoria del control geométrico no lineal con las estrategias establecidas en ingenieria de procesos.

Entre las características de los diseños propuestos para estimaciôn y control no lineal estân principalmente: (a) los diseños tienen un criterio de convergencia robusto, (b) el procedimiento de construcción es sistemático, (c) la técnica de sintonización es simple, sistemática, y con interpretación física, y (d) la estructura de estimabilidad/controlabilidad es un grado de libertad importante en el diseño. Resultando que la selección de las estructuras de estimabilidad (i.e. conjunto de salidas medidas, indices de observabilidad, y estados no observables) y de controlabilidad (i.e. conjunto de salidas de control, grados relativos, y estados no controlables) es crucial para el funcionamiento robusto de los estimadores y controladores. Ya que la selección de estas estructuras fue decisivo para mantener el 
balance entre desempeño y robustez, cuando existen errores en los parámetros del modelo, cambios en las entradas exógenas, y ruido en las mediciones.

Las implementaciones de los estimadores y controladores se ejemplificaron para la copolimerización en un reactor continuo de metil metacrilato - acetato de vinilo, con acetato de etilo como solvente y AIBN como iniciador. No obstante, la metodologia presentada es aplicable a cualquier sistema de reacción y cualquier tipo de polimerización, en reactores por lotes, semicontinuos, o continuos. El sistema de reacción metil metacrilato - acetato de vinilo, se seleccionó debido a que los monómeros muestran diferencias significativas en las reactividades y en el efecto gel. Lo cual, aunado con las caracteristicas de los reactores continuos de polimerización (comportamiento altamente no lineal, multiplicidad de estados estacionarios, fuerte acoplamiento entrada-salida, dificultades en la transferencia de calor por cambios en la viscosidad, etc.) hacen que las condiciones de operación sean severas, y por lo tanto mantener el compromiso entre desempeño y robustez de los estimadores/controladores se convierte en un reto, que es bien ilustrado con los resultados mostrados en esta tesis.

Comparando el estimador no lineal propuesto con el FEK (el cual ha sido el estimador más ampliamente usado en procesos químicos), el estimador propuesto muestra ventajas sobre el FEK. Ya que el FEK sólo se puede implementar para plantas observables, no explota la interpretación física del proceso en particular para su construcción-sintonización, no tiene un criterio de convergencia que garantice a priori el funcionamiento del mismo, el número de ganancias a ajustar es mayor al número de ganancias requeridas para el estimador no lineal, y no tiene una técnica de sintonización sistemática.

Comparando los controladores no lineales propuestos (un controlador con retroalimentación de mediciones donde las salidas medidas son iguales a las salidas de control, y otro controlador donde dichas salidas son diferentes) con otros controladores reportados en la literatura se obtuvieron mejores resultados, en cuanto a velocidad de convergencia. Un controlador lineal (como el presentado por Congalidis et al., 1985) converge en $\approx 18 \bar{\theta}_{r}\left(\bar{\theta}_{r}\right.$ es el tiempo nominal de residencia del reactor), un controlador no lineal (exacto) con retroalimentación de estados (como el presentado por Padilla et al., 1995) alcanza convergencia en $\approx 4 \bar{\theta}_{r}$, y un controlador avanzado [como el presentado por Padilla y Alvarez (1997), el cual es obtenido a partir de la linealización del control exacto con retroalimentación de estados) converge en $\approx 6 \bar{\theta}_{r}$. Mientras que el controlador no lineal propuesto, con salidas medidas iguales a las salidas de control converge en $\approx 10 \bar{\theta}_{r}$, y con salidas medidas diferentes a las salidas de control converge en $\approx 6 \bar{\theta}_{r}$. 


\subsection{Otras aplicaciones $y$ recomendaciones para trabajo futuro}

En cuanto al esquema de estimación no lineal, recientemente se ha aplicado para abordar problemas de estimación de estados en: (i) un reactor continuo de homopolimerización en masa vía radicales libres (Alvarez y López, 1999; Alvarez, 2000; Alvarez et al., 1999, 2000), y (ii) un reactor continuo de copolimerización en solución via radicales libres, empleando mediciones de presión de vapor, densidad, temperatura, y volumen (López y Alvarez, 1996, 1997). Asimismo la metodologia es extendible para: (i) la estimación de parámetros del modelo, como se ha hecho en microemulsión (López-Serrano et. al., 1999a, b), y en polimerización por etapas (López, 2000), y (ii) el diseño de estimadores con mediciones discreto-retardadas, como se ha hecho en homopolimerización en solución (Hernández y Alvarez, 2000) y en polimerización por etapas (López et al., 2000).

Entre los temas de estimación aún por estudiar o en investigación actual están: (i) establecer el criterio de convergencia del estimador no local semiglobal (Alvarez et al., 1999, 2000), (ii) el diseño de estimadores basados en la combinación de mediciones discretas y continuas (Hernández, 2000), y (iii) empleo de estimadores en línea para la detección de fallas (Hernández et al., 2000)

Con respecto al esquema de control no lineal con retroalimentación de mediciones (basado en un estimador), éste ha sido empleado en el control de un reactor de homopolimerización en masa: (i) considerando una acción integral en el controlador (Alvarez, 1996), y (ii) considerando la acción integral en el estimador (Alvarez y López, 2000). Existen aún hay diversos aspectos por estudiar: (i) el criterio de convergencia robusto del controlador no lineal propuesto (Alvarez et al., 2000), (ii) el criterio de convergencia no local (o semiglobal); (iii) la justificación de convergencia del segundo esquema de control propuesto (donde las salidas informativas son diferentes a las salidas reguladas, y se incluye un doble integrador). 


\section{NOMENCLATURA}

A Area de transferencia de calor, $\mathrm{m}^{2}$

A() Matriz de desacoplamiento.

$a_{\mathrm{h}} \quad$ Constante de la función del coeficiente de transferencia de calor

$A(T) \quad$ Funcionalidad de la viscosidad

$A_{\mathrm{v}} \quad$ Constante de la función de viscosidad

$B \quad$ Funcionalidad de la viscosidad

$b_{\mathrm{h}} \quad$ Constante de la función del coeficiente de transferencia de calor

$B_{\mathrm{v}} \quad$ Constante de la función de viscosidad

$b_{\mathrm{ij}} \quad$ Constantes de la función del efecto gel

c Composición del monómero 1 en la mezcla polimérica, fracción molar

$C_{\mathrm{h}} \quad$ Constante de la función del coeficiente de transferencia de calor

$C_{\mathrm{p}} \quad$ Capacidad calorifica de la mezcla polimérica, $\mathrm{KJ} / \mathrm{Kg}{ }^{\circ} \mathrm{K}$

$C_{\mathrm{pi}} \quad$ Capacidad calorifica del $i$-ésimo monómero, $\mathrm{KJ} / \mathrm{Kg}{ }^{\circ} \mathrm{K}$

$C_{\mathrm{ps}} \quad$ Capacidad calorifica del $i$-ésimo solvente, $\mathrm{KJ} / \mathrm{Kg}{ }^{\circ} \mathrm{K}$

$C_{\mathrm{v}} \quad$ Constante de la función de viscosidad

$C_{\mathrm{x}} \quad$ Controlador con retroalimentación de estados

$C_{\mathrm{y}} \quad$ Controlador con retroalimentación de salidas (mediciones)

$d \quad$ Vector de entradas exógenas

$D_{i} \quad$ Diámetro del impulsor, $\mathrm{m}$

$D_{\mathrm{t}} \quad$ Diámetro del reactor, $\mathrm{m}$

$d_{\mathrm{h}} \quad$ Constante de la función del coeficiente de transferencia de calor.

$D_{\mathrm{n}, \mathrm{m}} \quad$ Concentración del polímero muerto con $\mathrm{n}$ unidades de $M_{1} \mathrm{y} \mathrm{m}$ unidades de $M_{2}, \mathrm{kmol} / \mathrm{m}^{3}$

$E_{\mathrm{i}} \quad$ Estimador correspondiente a la estructura $S_{\mathrm{i}}$

$\mathrm{E}(t) \quad$ Evolución de la planta

$E_{\mathrm{d}} \quad$ Energia de activación de disociación del iniciador, $\mathrm{KJ} / \mathrm{Kmol}$

$E_{\mathrm{pij}} \quad$ Energia de activación de propagación, $\mathrm{KJ} / \mathrm{Kmol}$

$E_{\mathrm{tij}} \quad$ Energia de activación de terminación, $\mathrm{KJ} / \mathrm{Kmol}$

$f \quad$ Factor de eficiencia del iniciador

$f_{\mathrm{i}} \quad$ Funcionalidades de la ecuación de estado

$g_{\mathrm{ij}} \quad$ Funcionalidades de la ecuación de estado

$g_{\text {tii }} \quad$ Factor del efecto gel del $i$-ésimo monómero

$\Delta H_{\mathrm{pij}} \quad$ Constante de velocidad de propagación, $\mathrm{KJ} / \mathrm{Kmol}$

$i \quad$ Concentración adimensional del iniciador.

I Concentración del iniciador, $\mathrm{kmol} / \mathrm{m}^{3}$

$I^{\mathrm{o}} \quad$ Concentración molar del iniciador en la alimentación, $\mathrm{kmol} / \mathrm{m}^{3}$ 
$k \quad$ Conductividad térmica, $\mathrm{KJ} / \mathrm{m} \min ^{\circ} \mathrm{K}$

$k_{\mathrm{d}} \quad$ Constante de velocidad de disociación del iniciador, $\min ^{-1}$

$k_{\mathrm{d}}{ }^{\circ} \quad$ Factor de frecuencia de disociación del iniciador, $\min ^{-1}$

$k_{\mathrm{ij}} \quad$ Constante de velocidad de formación de radicales primarios, $\mathrm{m}^{3} / \mathrm{Kmol} \mathrm{min}$

$k_{\mathrm{pij}} \quad$ Constante de velocidad de propagación, $\mathrm{m}^{3} / \mathrm{Kmol} \mathrm{min}$

$k_{\mathrm{pij}}{ }^{\mathrm{o}} \quad$ Factor de frecuencia de formación de radicales primarios, $\mathrm{m}^{3} / \mathrm{Kmol} \mathrm{min}$

$k_{\mathrm{tij}} \quad$ Constante de velocidad de terminación, $\mathrm{m}^{3} / \mathrm{Kmol} \mathrm{min}$

$k_{\mathrm{tij}}{ }^{\circ} \quad$ Factor de frecuencia de propagación, $\mathrm{m}^{3} / \mathrm{Kmol} \mathrm{min}$

$L \quad$ Longitud del reactor (altura), $m$

$M \quad$ Peso molecular promedio-peso, $\mathrm{Kg} / \mathrm{Kmol}$

$m_{\mathrm{i}} \quad$ Concentración adimensional del $i$-ésimo monómero.

$M_{\mathrm{i}} \quad$ Concentración del $i$-ésimo monómero, $\mathrm{kmol} / \mathrm{m}^{3}$

$M_{\mathrm{ie}} \quad$ Concentración en la alimentación del $i$-ésimo monómero, $\mathrm{kmol} / \mathrm{m}^{3}$

$M_{\mathrm{i}}^{\mathrm{o}} \quad$ Concentración molar del $i$-ésimo monómero puro, $\mathrm{kmol} / \mathrm{m}^{3}$

$m_{\mathrm{s}} \quad$ Fracción de sólidos en la mezcla polimérica, adimensional

$m_{i} \quad$ Peso molecular del $i$-ésimo monómero, $\mathrm{Kg} / \mathrm{Kmol}$

$m_{\mathrm{s}} \quad$ Peso molecular del solvente, $\mathrm{Kg} / \mathrm{Kmol}$

$N \quad$ Velocidad de agitación, RPM

$p \quad$ Conversión total en la mezcla polimérica, fracción molar

$P \quad$ Concentración total de los radicales activos que terminan con un radical $\mathrm{M}_{1}$, $\mathrm{kmol} / \mathrm{m}^{3}$

$p_{\mathrm{i}} \quad$ Concentración adimensional del $i$-ésimo monómero convertido a polimero.

$P_{\mathrm{i}} \quad$ Concentración del $i$-ésimo monómero convertido a polímero, $\mathrm{kmol} / \mathrm{m}^{3}$

$P_{\text {ie }} \quad$ Concentración en la alimentación del i-ésimo monómero convertido a polimero, $\mathrm{kmol} / \mathrm{m}^{3}$

$P_{\mathrm{i}}{ }^{\circ} \quad$ Concentración molar del $i$-ésimo polimero, $\mathrm{kmol} / \mathrm{m}^{3}$

$P_{\mathrm{n}, \mathrm{m}} \quad$ Concentración de la cadena polimérica activa con $\mathrm{n}$ unidades de $\mathrm{M}_{1}$ y $\mathrm{m}$ unidades de $M_{2}$ terminando con un radical activo $M_{1}, \mathrm{kmol} / \mathrm{m}^{3}$

$Q_{\mathrm{n}, \mathrm{m}} \quad$ Concentración de la cadena polimérica activa con $\mathrm{n}$ unidades de $\mathrm{M}_{1}$ y $\mathrm{m}$ unidades de $M_{2}$ terminando con un radical activo $M_{2}, \mathrm{kmol} / \mathrm{m}^{3}$

$Q \quad$ Concentración total de los radicales activos que terminan con un radical $\mathbf{M}_{2}$, $\mathrm{kmol} / \mathrm{m}^{3}$

$q \quad$ Flujo de descarga del reactor, $\mathrm{m}^{3} / \mathrm{min}$

$q_{\mathrm{i}} \quad$ Flujo de alimentación del $i$-ésimo monómero, $\mathrm{m}^{3} / \mathrm{min}$

$q_{\mathrm{s}} \quad$ Flujo de alimentación del $i$-ésimo solvente, $\mathrm{m}^{3} / \mathrm{min}$

$R \quad$ Concentración de los radicales primarios, $\mathrm{kmol} / \mathrm{m}^{3}$

$N_{\mathrm{Pr}} \quad$ Número adimensional de Prandalt.

$N_{\mathrm{Re}} \quad$ Número adimensional de Reynolds.

$R \quad$ Velocidad de producción de copolimero, $\mathrm{Kg} / \mathrm{h}$

$R_{\mathrm{e}} \quad$ Concentración en la alimentación de los radicales primarios, $\mathrm{kmol} / \mathrm{m}^{3}$ 


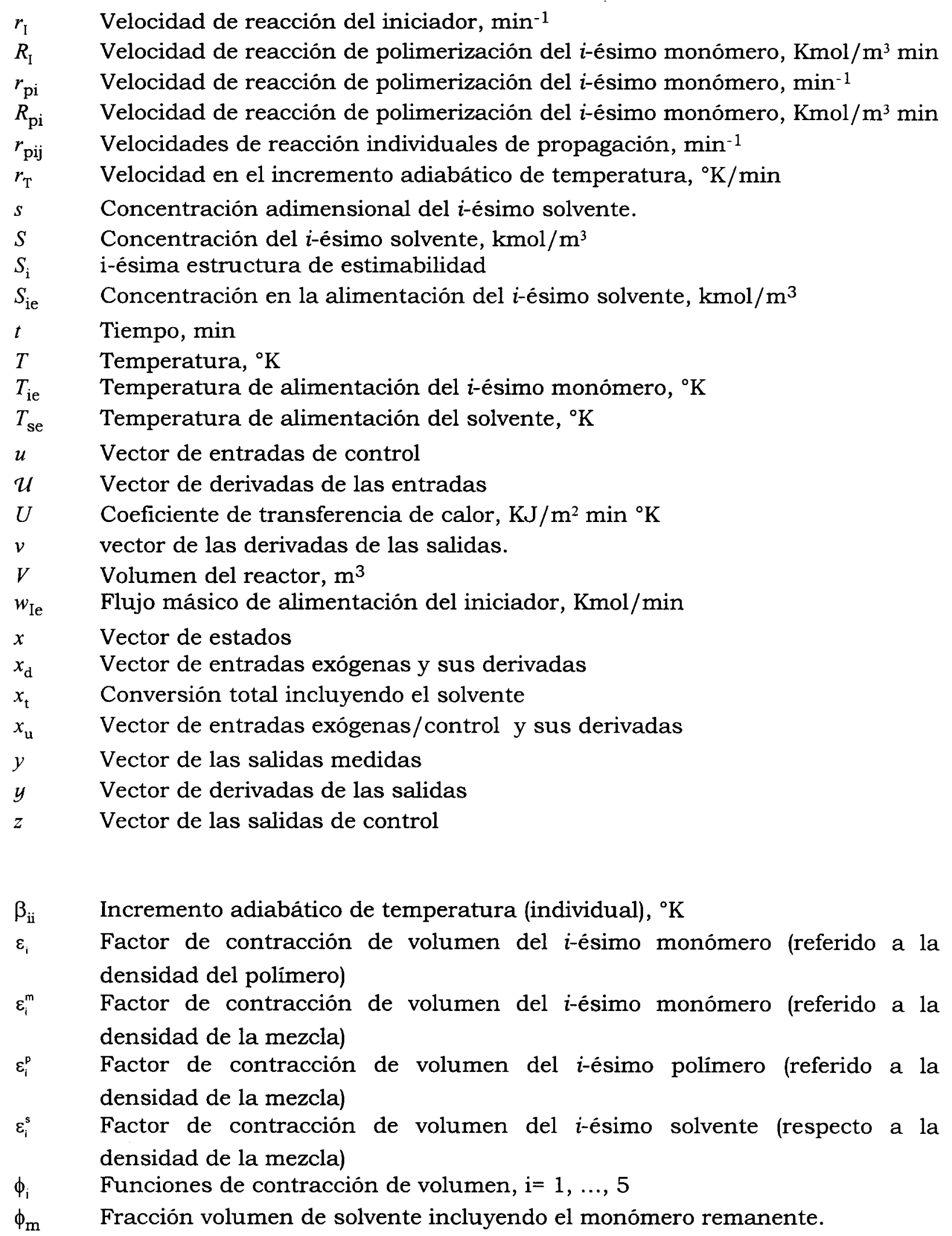


Velocidad de transferencia de calor entre el reactor y la chaqueta de enfriamiento, $\min ^{-1}$

Indice de refracción de la mezcla polimérica

Indice de refracción de la mezcla de monómeros y solvente

$\eta_{\mathrm{i}} \quad$ Indice de refracción del i-ésimo monómero

$\eta_{s} \quad$ Indice de refracción del solvente

$\kappa \quad$ Grado de estimabilidad de la planta

$\kappa_{c} \quad$ Grado relativo de la planta

$\kappa_{\mathrm{i}} \quad$ Indice de estimabilidad de la salida $\mathrm{y}_{\mathrm{i}}$

$\kappa_{i}^{c} \quad$ Grado relativo de la salida $\mathrm{y}_{\mathrm{i}}$

$\mu \quad$ Viscosidad de la mezcla polimérica, $\mathrm{Kg} / \mathrm{m} \mathrm{min}$

$\mu_{\mathrm{o}} \quad$ Viscosidad de referencia de la mezcla polimérica, $\mathrm{Kg} / \mathrm{m}$ s

$\mu_{0} \quad$ Momento cero de la distribución de longitud de cadena (CLD)

$\mu_{2} \quad$ Segundo momento de la distribución de longitud de cadena (CLD)

$\rho \quad$ Densidad de la mezcla polimérica, $\mathrm{Kg} / \mathrm{m}^{3}$

$\rho_{\mathrm{i}}^{\mathrm{m}} \quad$ Densidad del $i$-ésimo monómero puro, $\mathrm{Kg} / \mathrm{m}^{3}$

$\rho_{1}^{p} \quad$ Densidad del $i$-ésimo polímero, $\mathrm{Kg} / \mathrm{m}^{3}$

$\rho_{1}^{s} \quad$ Densidad del $i$-ésimo solvente, $\mathrm{Kg} / \mathrm{m}^{3}$

$\rho_{\mathrm{ie}} \quad$ Densidad del $i$-ésimo flujo de alimentación de monómero, $\mathrm{Kg} / \mathrm{m}^{3}$

$v_{i} \quad$ Incremento en el índice de refracción especifico del i-ésimo polímero 


\section{APÉNDICE A. Funcionalidades del modelo del reactor de copolimerización en solución}

\section{Concentraciones adimensionales}

$$
\begin{array}{ll}
m_{1}=\frac{M_{1}}{M_{1}^{o}}, & M_{1}^{o}=\frac{\rho_{1}^{m}}{m_{1}} \\
m_{2}=\frac{M_{2}}{M_{2}^{o}}, & M_{2}^{o}=\frac{\rho_{2}^{m}}{m_{2}} \\
p_{1}=\frac{P_{1}}{P_{1}^{o}}, & P_{1}^{o}=\frac{\rho_{1}^{p}}{m_{1}} \\
p_{2}=\frac{P_{2}}{P_{2}^{o}}, & P_{2}^{o}=\frac{\rho_{2}^{p}}{m_{2}} \\
s=\frac{S}{S^{o}}, & S^{o}=\frac{\rho_{s}}{m_{s}}
\end{array}
$$

\section{Cinética de copolimerización}

$$
\begin{aligned}
& r_{I}(i, T)=k_{d} i \\
& r_{1}\left(m_{1}, m_{2}, p_{1}, p_{2}, i, T\right)=m_{1}\left(k_{p 11} P+k_{p 21} Q\right) \\
& r_{2}\left(m_{1}, m_{2}, p_{1}, p_{2}, i, T\right)=m_{2}\left(k_{p 12} P+k_{p 22} Q\right) \\
& r_{p 11}\left(m_{1}, m_{2}, p_{1}, p_{2}, i, T\right)=k_{p 11} P m_{1} \\
& r_{p 21}\left(m_{1}, m_{2}, p_{1}, p_{2}, i, T\right)=k_{p 21} Q m_{1} \\
& r_{p 12}\left(m_{1}, m_{2}, p_{1}, p_{2}, i, T\right)=k_{p 12} P m_{2} \\
& r_{p 22}\left(m_{1}, m_{2}, p_{1}, p_{2}, i, T\right)=k_{p 22} Q m_{2} \\
& P\left(m_{1}, m_{2}, p_{1}, p_{2}, i, T\right)=\alpha Q \\
& Q\left(m_{1}, m_{2}, p_{1}, p_{2}, i, T\right)=\sqrt{\frac{2 f k_{d} I}{k_{t 11} \alpha^{2}+2 k_{t 12} \alpha+k_{t 22}}} \\
& \alpha\left(m_{1}, m_{2}, T\right)=\frac{k_{p 21} M_{1}}{k_{p 12} M_{2}}
\end{aligned}
$$




$$
\begin{aligned}
& k_{d}(T)=k_{d}^{o} \exp \left[-E_{d} /(R T)\right] \\
& k_{p i j}(T)=k_{p i j}^{o} \exp \left[-E_{p i j} /(R T)\right], \quad \mathrm{i}=1,2 \\
& k_{t i i}\left(m_{1}, m_{2}, p_{1}, p_{2}, T\right)=k_{t i j}^{o} \exp \left[-E_{t i i} /(R T)\right] g_{t i}, \quad \mathrm{i}=1,2 \\
& k_{t 12}\left(m_{1}, m_{2}, p_{1}, p_{2}, T\right)=\sqrt{k_{t 1} k_{t 22}} \\
& g_{t i}\left(m_{1}, m_{2}, p_{1}, p_{2}, T\right)=\exp \left[-\left(b_{i 1}+b_{i 2} T\right) x_{T}\right], \quad \mathrm{i}=1,2 \\
& x_{t}\left(m_{1}, m_{2}, p_{1}, p_{2}\right)=\frac{p_{1} P_{1}^{o}+p_{2} P_{2}^{o}}{m_{1} M_{1}^{o}+m_{2} M_{2}^{o}+p_{1} P_{1}^{o}+p_{2} P_{2}^{o}+\left(1-m_{1}-m_{2}-p_{1}-p_{2}\right) S^{o}}
\end{aligned}
$$

\section{Contracción de volumen}

$$
\begin{aligned}
& \varepsilon_{1}=1-\rho_{1}^{m} / \rho_{1}^{P} \\
& \varepsilon_{2}=1-\rho_{2}^{m} / \rho_{2}^{P} \\
& \varepsilon_{i}^{m}=1-\rho_{i}^{m} / \rho \\
& \varepsilon_{i}^{p}=1-\rho_{i}^{p} / \rho \\
& \varepsilon^{s}=1-\rho^{s} / \rho \\
& \phi_{1}=\left(1-\varepsilon_{1}^{m}\right)\left(1-\varepsilon_{1} m_{1 e}\right) /\left(1-\varepsilon_{1}\right)+\varepsilon_{1}^{m} m_{1 e}+\varepsilon_{1}^{p} p_{1 e} \\
& \phi_{2}=\left(1-\varepsilon_{2}^{m}\right)\left(1-\varepsilon_{2} m_{2 e}\right) /\left(1-\varepsilon_{2}\right)+\varepsilon_{2}^{m} m_{2 e}+\varepsilon_{2}^{p} p_{2 e} \\
& \phi_{3}=1+\varepsilon^{s}\left(s_{1 e}-1\right)
\end{aligned}
$$

\section{Viscosidad de la mezcla polimérica}

$$
\begin{aligned}
& \mu\left(p_{1}, p_{2}, T\right)=\mu_{o} \exp \left[-\frac{2.3 \phi_{m}}{A(T)+B \phi_{m}}\right]^{C_{v}} \\
& \mu_{o}(T)=A_{v}(T-273)^{-B_{v}} \\
& \phi_{m}\left(p_{1}, p_{2}\right)=1-p_{1}+p_{2} \\
& A(T)=A_{A}+B_{A}\left(1-\frac{T}{T_{g}}\right)+C_{A}\left(1-\frac{T}{T_{g}}\right)^{2}
\end{aligned}
$$




\section{Velocidad de transferencia de calor}

$$
\begin{aligned}
& r_{T}\left(m_{1}, m_{2}, p_{1}, p_{2}, i, T, V\right)=\sum \sum \beta_{i j} r_{p i j} \\
& \gamma\left(m_{1}, m_{2}, p_{1}, p_{2}, T, V\right)=U A /\left(\rho V C_{p}\right) \\
& \beta_{i j}\left(m_{1}, m_{2}, p_{1}, p_{2}\right)=\left(-\Delta H_{p i j}\right) M_{j}^{o} /\left(\rho C_{p}\right) \\
& U\left(m_{1}, m_{2}, p_{1}, p_{2}, T\right)=a_{h}\left(k / D_{i}\right)\left(N_{R e}\right)^{b_{h}}\left(N_{P r}\right)^{c_{h}}\left(\mu / \mu_{w}\right)^{d_{h}}, \\
& N_{R e}\left(m_{1}, m_{2}, p_{1}, p_{2}, T\right)=D_{i}^{2} N \rho / \mu, \quad N_{P r}\left(m_{1}, m_{2}, p_{1}, p_{2}, T\right)=C_{p} \mu / k \\
& q_{h e}\left(m_{1}, m_{2}, p_{1}, p_{2}, T, V\right)=\left(q_{1} \rho_{1} C_{p 1} T_{1 e}+q_{2} \rho_{2} C_{p 2} T_{2 e}+q_{s} \rho_{s} C_{p s} T_{s e}\right) /\left(\rho V C_{p}\right) \\
& q_{h}\left(m_{1}, m_{2}, p_{1}, p_{2}, T, V\right)=\left(q_{1} \rho_{1}+q_{2} \rho_{2}+q_{s} \rho_{s}\right) T /(\rho V)
\end{aligned}
$$

Momentos de la distribución de longitud de cadena (DLC)

$$
\begin{aligned}
& r_{\mu_{0}}=1 / 2 k_{t 11} \lambda_{01}{ }^{2}+k_{t 12} \lambda_{01} \lambda_{02}+1 / 2 k_{t 22} \lambda_{02}{ }^{2} \\
& r_{\mu_{1}}=k_{t 11} \lambda_{01} \lambda_{11}+k_{t 12}\left(\lambda_{01} \lambda_{12}+\lambda_{02} \lambda_{11}\right)+k_{t 22} \lambda_{02} \lambda_{12} \\
& r_{\mu_{2}}=k_{t 11}\left(\lambda_{11}{ }^{2}+\lambda_{01} \lambda_{21}\right)+k_{t 12}\left(\lambda_{22} \lambda_{01}+\lambda_{21} \lambda_{02}+2 \lambda_{11} \lambda_{12}\right)+k_{t 22}\left(\lambda_{12}{ }^{2}+\lambda_{02} \lambda_{22}\right) \\
& \lambda_{i 1}=\sum_{n=0}^{\infty} \sum_{m=0}^{\infty}\left(n M_{w 1}+m M_{w 2}\right)^{i} P_{n, m} \\
& \lambda_{i 2}=\sum_{n=0}^{\infty} \sum_{m=0}^{\infty}\left(n M_{w 1}+m M_{w 2}\right)^{i} Q_{n, m} \\
& \mu_{i}=\sum_{n=0}^{\infty} \sum_{m=0}^{\infty}\left(n M_{w 1}+m M_{w 2}\right)^{i} D_{n, m}
\end{aligned}
$$


Modelo del reactor en la forma: $\quad \dot{x}=f_{r}(x, d, b)+G_{r}(x, d, b) u$

$$
\begin{aligned}
& f_{r}(x, d, b)=\left[\begin{array}{c}
-r_{1}-q_{s} \phi_{3} m_{1} / V-\left(r_{1} \phi_{4}+r_{2} \phi_{5}\right) m_{1} \\
-r_{2}-q_{s} \phi_{3} m_{2} / V-\left(r_{1} \phi_{4}+r_{2} \phi_{5}\right) m_{2} \\
r_{1}\left(1-\varepsilon_{1}\right)-q_{s} \phi_{3} p_{1} / V-\left(r_{1} \phi_{4}+r_{2} \phi_{5}\right) p_{1} \\
r_{2}\left(1-\varepsilon_{2}\right)-q_{s} \phi_{3} p_{2} / V-\left(r_{1} \phi_{4}+r_{2} \phi_{5}\right) p_{2} \\
-r_{I}+\left(w_{l e} / I^{o}-q_{s} \phi_{3} i\right) / V-\left(r_{1} \phi_{4}+r_{2} \phi_{5}\right) i \\
r_{T}-\gamma T+q_{s} \rho_{s}\left(C_{p s} T_{s e} / C_{p}-T\right) /(\rho V) \\
q_{s} \phi_{3}+\left(r_{1} \phi_{4}+r_{2} \phi_{5}\right) V \\
r_{\mu_{0}}-q_{s} \phi_{3} \mu_{0} / V-\left(r_{1} \phi_{4}+r_{2} \phi_{5}\right) \mu_{0} \\
r_{\mu_{2}}-q_{s} \phi_{3} \mu_{2} / V-\left(r_{1} \phi_{4}+r_{2} \phi_{5}\right) \mu_{2}
\end{array}\right]:=\left[\begin{array}{c}
f_{r 1} \\
f_{r 2} \\
f_{r 3} \\
f_{r 4} \\
f_{r 5} \\
f_{r 6} \\
f_{r 7} \\
f_{r 8} \\
f_{r 9}
\end{array}\right] \\
& G_{r}(x, d, b)=\left[\begin{array}{cccc}
\left(m_{1 e}-\phi_{1} m_{1}\right) / V & -\phi_{2} m_{1} / V & 0 & 0 \\
-\phi_{1} m_{2} / V & \left(m_{2 e}-\phi_{2} m_{2}\right) / V & 0 & 0 \\
\left(p_{1 e}-\phi_{1} p_{1}\right) / V & -\phi_{2} p_{1} / V & 0 & 0 \\
-\phi_{1} p_{2} / V & \left(p_{2 e}-\phi_{2} p_{2}\right) / V & 0 & 0 \\
-\phi_{1} i / V & -\phi_{2} i / V & 0 & 0 \\
\rho_{1}\left(C_{p 1} T_{1 e} / C_{p}-T\right) /(\rho V) & \rho_{2}\left(C_{p 2} T_{2 e} / C_{p}-T\right) /(\rho V) & \gamma & 0 \\
\phi_{1} & \phi_{2} & 0 & 1 \\
\left(\mu_{0 e}-\phi_{1} \mu_{0}\right) / V & \left(\mu_{0 e}-\phi_{2} \mu_{0}\right) / V & 0 & 0 \\
\left(\mu_{2 e}-\phi_{1} \mu_{2}\right) / V & \left(\mu_{2 e}-\phi_{2} \mu_{2}\right) / V & 0 & 0
\end{array}\right] \\
& G_{r}(x, d, b):=\left[\begin{array}{c}
g_{r 1} \\
\vdots \\
g_{r 9}
\end{array}\right]=\left[\begin{array}{cccc}
g_{r 11} & g_{r 21} & g_{r 31} & g_{r 41} \\
\vdots & \vdots & \vdots & \vdots \\
g_{r 19} & g_{r 29} & g_{r 39} & g_{r 49}
\end{array}\right]
\end{aligned}
$$




\section{APÉNDICE B. Parámetros para el reactor de copolimerización de MMA-AV}

\section{Propiedades fisicoquímicas de los monómeros}

(Brandrup e Immergut, 1989)

$$
\begin{array}{llll}
M_{1}=100.11 \mathrm{Kmol} / \mathrm{Kg} & C_{\mathrm{p} 1}=1.80 \mathrm{KJ} / \mathrm{Kg}^{\circ} \mathrm{K} & \\
M_{2}=86.09 \mathrm{Kmol} / \mathrm{Kg} & C_{\mathrm{p} 2}=1.97 \mathrm{KJ} / \mathrm{Kg}^{\circ} \mathrm{K} & \\
S & =88.10 \mathrm{Kmol} / \mathrm{Kg} & C_{\mathrm{ps}}=1.91 \mathrm{KJ} / \mathrm{Kg}^{\circ} \mathrm{K} & \\
& & & \\
\rho_{1}{ }^{0} & =950.0 \mathrm{Kg} / \mathrm{m}^{3} & M_{1}{ }^{0}=9.48960 \mathrm{kmol} / \mathrm{m}^{3} & \eta_{1}=1.4130 \\
\rho_{2}{ }^{0} & =932.0 \mathrm{Kg} / \mathrm{m}^{3} & M_{2}{ }^{0}=10.82588 \mathrm{kmol} / \mathrm{m}^{3} & \eta_{2}=1.3959 \\
\rho_{1}{ }^{\mathrm{p}}=1170.0 \mathrm{Kg} / \mathrm{m}^{3} & P_{1}{ }^{0}=11.68714 \mathrm{kmol} / \mathrm{m}^{3} & \eta_{\mathrm{s}}=1.3700 \\
\rho_{2}{ }^{\mathrm{p}}=1190.0 \mathrm{Kg} / \mathrm{m}^{3} & P_{2}{ }^{\circ}=13.82274 \mathrm{kmol} / \mathrm{m}^{3} & v_{1}=0.000118 \mathrm{~m}^{3} / \mathrm{Kg} \\
\rho_{\mathrm{s}}{ }^{0} & =901.0 \mathrm{Kg} / \mathrm{m}^{3} & S^{0}=10.22701 \mathrm{kmol} / \mathrm{m}^{3} & v_{2}=0.000087 \mathrm{~m}^{3} / \mathrm{Kg}
\end{array}
$$

\section{Constantes cinéticas}

(Brandrup e Immergut, 1989)

$$
\begin{array}{rlrl}
k_{\mathrm{d}}{ }^{\circ}=5.95 \times 10^{13} \mathrm{~m}^{3} / \mathrm{Kmol} \mathrm{s} & E_{\mathrm{d}}=123846.4 \mathrm{KJ} / \mathrm{Kmol} \\
k_{\mathrm{p} 11}{ }^{0}=7.00 \times 10^{6} \mathrm{~m}^{3} / \mathrm{Kmol} \mathrm{s} & E_{\mathrm{p} 11}=26359.2 \mathrm{KJ} / \mathrm{Kmol} \\
k_{\mathrm{p} 22}{ }^{0}=1.30 \times 10^{8} \mathrm{~m}^{3} / \mathrm{Kmol} \mathrm{s} & E_{\mathrm{p} 22}=26359.2 \mathrm{KJ} / \mathrm{Kmol} \\
k_{\mathrm{t} 11}{ }^{\circ}=1.76 \times 10^{9} \mathrm{~m}^{3} / \mathrm{Kmol} \mathrm{s} & E_{\mathrm{t} 11}=11715.2 \mathrm{KJ} / \mathrm{Kmol} \\
k_{\mathrm{t} 22}{ }^{\circ}=2.80 \times 10^{10} \mathrm{~m}^{3} / \mathrm{Kmol} \mathrm{s} & E_{\mathrm{t} 22}=11715.2 \mathrm{KJ} / \mathrm{Kmol} \\
-\Delta H_{11}=56484.0 \mathrm{KJ} / \mathrm{Kmol} & & \\
-\Delta H_{22}=87864.0 \mathrm{KJ} / \mathrm{Kmol} & b_{11}=54.5 \\
-\Delta H_{12}=87864.0 \mathrm{KJ} / \mathrm{Kmol} & b_{12}=-0.13 \\
-\Delta H_{21}=56484.0 \mathrm{KJ} / \mathrm{Kmol} & b_{21}=32.2 \\
r_{1}=26.0 & b_{22}=-0.08 \\
f=0.60 &
\end{array}
$$


Constantes para la expresión de viscosidad

(Brandrup e Immergut, 1989)

$$
\begin{array}{ll}
B & =0.03 \\
A_{\mathrm{v}} & =4.14 \times 10^{25} \\
B_{\mathrm{v}} & =9.6763 \\
C_{\mathrm{v}} & =1.99 \\
A_{\mathrm{A}} & =0.1678 \\
B_{\mathrm{A}} & =0.0000 \\
C_{\mathrm{A}} & =-1.23577
\end{array}
$$

\section{Constantes para la transferencia de calor}

(Bondy y Lippa, 1983)

$$
\begin{aligned}
& a_{\mathrm{h}}=0.54 \text { si } \operatorname{Re}<400,0 \quad a_{\mathrm{h}}=0.74 \text { si } \operatorname{Re}>400 \\
& b_{\mathrm{h}}=2 / 3 \\
& c_{\mathrm{h}}=1 / 3 \\
& d_{\mathrm{h}}=0.14
\end{aligned}
$$

\section{Dimensiones del reactor}

Diämetro del reactor $\left(D_{\mathrm{T}}\right) \quad=1.3 \mathrm{~m}$

Diámetro del impulsor $\left(D_{\mathrm{i}}\right) \quad=0.7 \mathrm{~m}$

Velocidad de agitación $(N)=140 \mathrm{RPM}$

Volumen del reactor $(V) \quad=2.0 \mathrm{~m}^{3}$

Longitud recta del reactor $(\mathrm{L})=1.51 \mathrm{~m}$ 


\section{APÉNDICE C. Herramientas de sistemas dinámicos no lineales.}

La definiciones, teoremas, y lemas que se dan en este apéndice se han tomado de la siguiente literatura: Kwakernaak y Sivan (1972), Vidyasagar (1978), Stephanopoulos (1984), y Slotine (1991); y se incluyen para facilitar la lectura de la tesis.

\section{Derivada direccional}

La i-ésima derivada direccional recursiva $L_{\mathrm{f}}^{\mathrm{i}} \alpha$ del campo escalar variante en tiempo $\alpha(x, t)$ a lo largo del campo vectorial $f(x, t)$ esta dada por:

$$
\begin{array}{lll}
L_{f}^{i+1} \alpha=L_{f}\left(L_{f}^{i} \alpha\right), & i \geq 1 ; & L_{f}^{0} \alpha=\alpha \\
L_{f} \alpha=\alpha_{x} f+\alpha_{t}, & \alpha_{x}=\frac{\partial \alpha}{\partial x}, & \alpha_{t}=\frac{\partial \alpha}{\partial t}
\end{array}
$$

\section{Continuidad (Slotine, 1991)}

Intuitivamente, una función $f(x, t)$ es continua si la función se mantiene dentro de una vecindad cercana para pequeños intervalos de $x$. Su definición formal es como sigue.

Definición C.1 (Continuidad). La función $f(x, t)$ es continua en $x_{\mathrm{o}} \in X$ si, para todo $\varepsilon>0$, existe un $\delta\left(\varepsilon, x_{0}\right)$ tal que

$$
\left\|f(x, t)-f\left(x_{o}, t\right)\right\|<\varepsilon, \quad\left\|x-x_{o}\right\|<\delta .
$$

Se dice que $f$ es continua si es continua para todo $x \in X$. Finalmente, $f$ es uniformemente-continua si, para todo $\varepsilon>0$, existe un $\delta(\varepsilon)$ tal que

$$
\|f(x, t)-f(y, t)\|<\varepsilon, \quad\|x-y\|<\delta .
$$

La principal diferencia entre continuidad y continuidad-uniforme es que en éste último caso $\delta$ sólo depende $\varepsilon$ y no de $x$.

Teorema C.1 (Unicidad de soluciones). Si la función $f(x, t)$ es continua en $t$, y existe una constante $L$ estrictamente positiva tal que

$$
\left\|f\left(x_{2}, t\right)-f\left(x_{1}, t\right)\right\| \leq L\left\|x_{2}-x_{1}\right\|
$$


para todo $x_{1}$ y $x_{2}$ en una vecindad finita alrededor del origen, y para todo $t$ en el intervalo $\left(t_{0}, t_{0}+T\right)$ (con $T>0$ ); entonces $\dot{x}=f(x, t)$ tiene una solución única $x(t)$ para estados iniciales e intervalos de tiempo suficientemente pequeños.

La condición (C.4) es llamada condición de Lipschitz y $L$ es conocida como constante de Lipschitz. Si (C.4) se cumple, entonces se dice que $f$ es localmente Lipschitzcontinua en $x$ con respecto a $t$. Además, la satisfacción de una condición de Lipschitz no implica la continuidad (local) de $f$ en términos de $x$. Inversamente, si $f$ tiene un jacobiano $(\partial f / \partial x)$ localmente continuo y acotado con respecto a $x$, entonces $\mathrm{f}$ es localmente Lipschitz-continua. Cuando la condición de Lipschitz se satisface para cualquier $x_{1}$ y $x_{2}$ en el espacio de estados, entonces se dice que $f$ es globalmente Lipschitz-continua.

Definición C.2 (Continuidad Lipschitz). El mapa $\phi(x, u, b)$ es L(Lipschitz)-continuo a lo largo de la trayectoria $\mathrm{N}(t, b)$, si existe un conjunto de constantes positivas $\left\{D_{\mathrm{x}}, D_{\mathrm{u}}\right.$, $\left.D_{\mathrm{b}}\right\}$ tal que, en la vecindad de $\mathrm{N}(t, b), \phi$ esta acotada como sigue

$$
\left\|\phi\left(\hat{x}, \hat{x}_{u}, \beta\right)-\phi\left(x, x_{u}, b\right)\right\| \leq D_{x}\|\hat{x}-x\|+D_{u}\left\|\hat{x}_{u}-x_{u}\right\|+D_{b}\|\beta-b\| .
$$

\section{Invertibilidad}

Definición C.3 (Invertibilidad robusta). El mapa L-continuo $\phi(x, u, r)$ es Rx-invertible (robustamente invertible para $\mathrm{x}$ ) alrededor de $\mathrm{N}(t, b)$, si existe un mapa inverso Lcontinuo $\phi^{-1}$ tal que

$$
\phi^{-1}\{\phi[x(t), u(t), b], u(t), b\}=x(t), \quad t \geq 0 .
$$

\section{Estabilidad (Kwakernaak y Sivan, 1972)}

Sea $x_{0}(t)=\theta_{x}\left[t, t_{o}, x_{o}\right]$ la solución nominal del sistema no autónomo

$$
\dot{x}=f(x, t)
$$

con la condición inicial $\mathbf{x}\left(\mathbf{t}_{\mathrm{o}}\right)=\mathrm{x}_{\mathrm{o}}$; y sea $\bar{x}$ el vector de estados estacionarios o puntos de equilibrio definidos por

$$
f(\bar{x}, t) \equiv 0 \quad \forall t \geq 0
$$

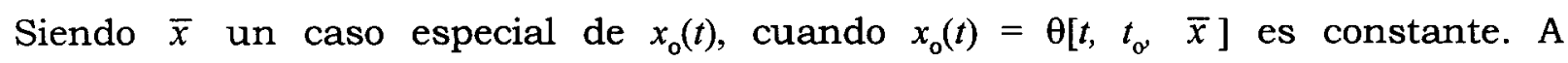
continuación se definen los conceptos de estabilidad/inestabilidad en el sentido de Lyapunov, estabilidad asintótica, y estabilidad exponencial. 
Definición C.4 (Estabilidad Lyapunov). La solución nominal $x_{\mathrm{o}}(t)$ es estable en el sentido de Lyapunov si, para cualquier $t_{\mathrm{o}}$ y cualquier $\varepsilon>0$, existe un $\delta(\varepsilon, t)>0$ tal que $\left\|x\left(t_{o}\right)-x_{o}\left(t_{o}\right)\right\| \leq \delta$ implica

$$
\left\|x(t)-x_{o}(t)\right\|<\varepsilon, \quad \forall t \geq 0
$$

Definición C.5 (Estabilidad asintótica). La solución nominal es A(asintóticamente)estable si

(a) es estable en el sentido de Lyapunov, y

(b) para todo $t_{\mathrm{o}}$ existe un $\rho\left(t_{\mathrm{o}}\right)>0$ tal que $\left\|x\left(t_{o}\right)-x_{o}\left(t_{o}\right)\right\|<\rho$ implica

$$
\left\|x(t)-x_{o}(t)\right\| \rightarrow 0, \quad \text { cuando } t \rightarrow \infty \text {. . }
$$

Definición C.6 (Estabilidad exponencial). El sistema no autónomo $\dot{x}=f(x, t)$ es E(exponencialmente)-estable si existen dos constantes positivas $\left(a_{\mathrm{x}}, \lambda_{\mathrm{x}}\right)$ tal que

$$
\|x(t)\| \leq a_{x} e^{-\lambda_{x}\left(t-t_{o}\right)}\left\|x\left(t_{o}\right)\right\|, \quad t \geq t_{o} \quad \bullet \text { (C.10) }
$$

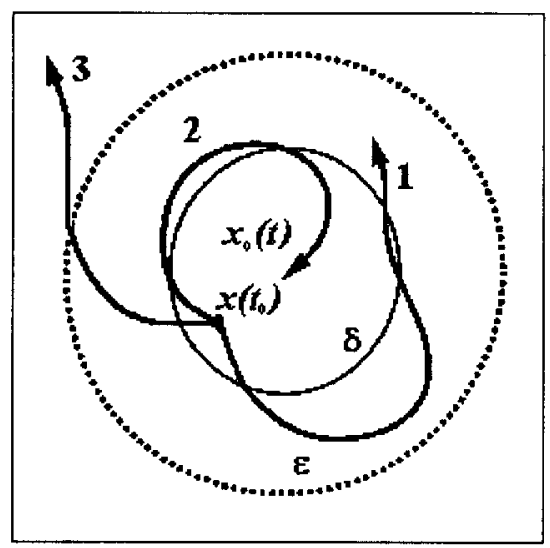

Figura C.1 Estabilidad de un punto:

(1) estable, (2) asintóticamente estable, (3) inestable 


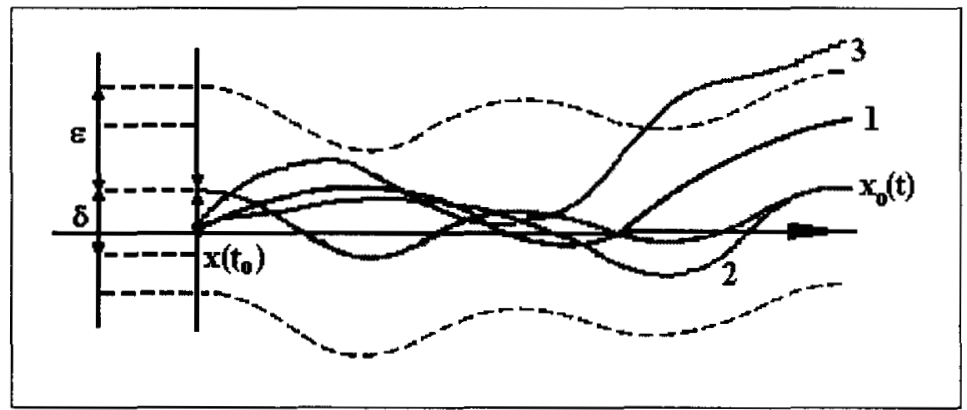

Figura C.2 Estabilidad de una trayectoria:

(1) estable, (2) asintóticamente estable, (3) inestable

\section{Teoremas conversos de Lyapunov (Slotine, 1991)}

Para el sistema no autónomo de la forma

$$
\dot{x}=f(x, t)
$$

siempre existen funciones de Lyapunov $V(x, t)$ si el sistema es estable, de acuerdo a los siguientes teoremas conversos.

Teorema C.2 (Estabilidad). Si el origen de (C.11) es estable, entonces existe una función $V(x, t)$ positiva definida con una derivada no positiva.

Teorema C.3 (Estabilidad asintótica). Si el punto de equilibrio en el origen es asintóticamente estable, entonces existe una función $V(x, t)$ positiva definida y decreciente con una derivada negativa definida.

Teorema C.4 (Estabilidad exponencial). Si la función $f(x, t)$ en (C.11) tiene primeras derivadas parciales continuas y acotadas con respecto a $x$ y $t$, para todo $x \in B_{\mathrm{r}}$ y para todo $t \geq 0$, entonces el punto de equilibrio en el origen es exponencialmente estable si, y solo si, existe una función $V(x, t)$ y cuatro constantes estrictamente positivas $\left(\alpha_{1}\right.$, $\alpha_{2}, \alpha_{3}, \alpha_{4}$ ) tal que

$$
\begin{gathered}
\alpha_{1}\|x\|^{2} \leq V(x, t) \leq \alpha_{2}\|x\|^{2} \\
\dot{V} \leq-\alpha_{3}\|x\|^{2} \\
\left\|\frac{\partial V}{\partial x}\right\| \leq \alpha_{4}\|x\|
\end{gathered}
$$


6. Lema de Bellman-Gronwall (Slotine, 1991; Vidyasagar, 1978)

En el análisis de estabilidad o convergencia de sistemas dinámicos, un problema frecuente es el establecimiento de limites de ciertas señales, por ejemplo, se puede manipular relaciones de variables dentro de una desigualdad integral de la forma:

$$
y(t) \leq \int_{0}^{t} a(\tau) y(\tau) d \tau+b(t)
$$

donde $y(t)$, la variable concerniente, aparece en ambos lados de la desigualdad. El problema consiste en obtener un limite explícito de la magnitud de $y$ a partir de la desigualdad anterior. Para este propósito se puede emplear el lema de BellmanGronwall.

Lema C.1 (Bellman-Gronwall). Sea una variable $y(t)$ que satisface (C.15), siendo $a(t)$ y $b(t)$ funciones reales conocidas. Entonces,

$$
y(t) \leq \int_{0} a(\tau) b(\tau) e^{\int_{\tau}^{t} a(r) d r} d \tau+b(t)
$$

Si $b(t)$ es diferenciable, entonces

$$
y(t) \leq b(0) e^{\int_{0}^{t} a(\tau) d \tau}+\int_{0} \dot{b}(\tau) e^{\int_{\tau}^{t} a(r) d r} d \tau
$$

En particular, si $b(t)$ es constante, simplemente se tiene

$$
y(t) \leq b(0) e^{\int_{0}^{t} a(\tau) d \tau}
$$

Prueba del Lema C.1. La prueba consiste en definir una nueva variable, para transformar la desigualdad integral (C.15) en una ecuación diferencial, la cual puede ser fácilmente resuelta.

Sea la nueva variable, $v(t)$, y su derivada:

$$
\begin{gathered}
\nu(t)=\int_{0} a(\tau) y(\tau) d \tau \\
\dot{v}=a(\tau) y(\tau)
\end{gathered}
$$

Sustituyendo (C.20) en la desigualdad (C.15) se obtiene:

$$
\begin{gathered}
\dot{v}=a(\tau) y(\tau) \leq a(t) \int_{0} a(\tau) y(\tau) d \tau+a(t) b(t) \\
\dot{v}=a(t) y(t) \leq a(t) v(t)+a(t) b(t) \\
y(t) \leq v(t)+b(t)
\end{gathered}
$$

Sea la función no positiva, $s(t)$ :

$$
s(t)=a(t) y(t)-a(t) v(t)-a(t) b(t)
$$


Entonces, usando las ecuaciones (C.20) y (C.22), v(t) satisface

$$
\dot{v}-a(t) v(t)=a(t) b(t)+s(t)
$$

Resolviendo ésta última, con la condición inicial $v(0)=0$, se obtiene

$$
v(t)=\int_{0}^{\int^{\int_{\tau} a(r) d r}}[a(\tau) b(\tau)+s(\tau)] d \tau
$$

Como s(.) es una función no positiva, entonces

$$
v(t) \leq \int_{0}^{t} e^{\int_{\tau}^{t} a(r) d r} a(\tau) b(\tau) d \tau
$$

Sustituyendo este último resultado (C.25) en la desigualdad (C.21), se llega a:

$$
y(t) \leq \int_{0}^{t} e^{\int_{\tau}^{t} a(r) d r} a(\tau) b(\tau) d \tau+b(t)
$$

la cual corresponde a la ecuación (C.16) del Lema de Bellman-Gronwall.

En el caso de que $b(t)$ sea diferenciable, la integral del lado derecho de la desigualdad de la ecuación (C.26) se obtiene empleando integración por partes:

$$
\int u d v=u v-\int v d u
$$

si $u=b(\cdot), d v=\int_{0} e^{\int_{\tau}^{t} a(r) d r} a(\tau) d \tau \Rightarrow d u=\dot{b}(\tau) d \tau, v=e^{\int_{\tau}^{t} a(r) d r}\left\{\begin{array}{r}r=t \\ h=0\end{array}\right.$, y se obtiene:

$$
\begin{aligned}
& \int_{0}^{t} e^{\int_{\tau}^{\prime} a(r) d r} a(\tau) b(\tau) d \tau=-b(t) e^{\int_{\tau}^{t} a(r) d r}{ }_{\tau=0}^{\tau=t}+\int_{0}^{c} \dot{b}(\tau) e^{\int_{\tau}^{\prime} a(r) d r} d \tau \\
& \int_{0} e^{\int_{\tau}^{\prime} a(r) d r} a(\tau) b(\tau) d \tau=-b(t) e^{\int^{\prime} f(t) d r}+b(0) e^{\int_{0}^{\prime} a(r) d r}+\int_{0}^{t} \dot{b}(\tau) e^{\int_{\tau}^{\prime} a(r) d r} d \tau
\end{aligned}
$$

Sustituyendo (C.27) en (C.26):

$$
\begin{gathered}
y(t) \leq-b(t)+b(0) e^{\int_{0}^{t} a(r) d r}+\int_{0}^{b} \dot{b}(\tau) e^{\int_{\tau}^{t} a(r) d r} d \tau+b(t) \\
y(t) \leq b(0) e^{\int_{0}^{t} a(r) d r}+\int_{0}^{t} \dot{b}(\tau) e^{\int_{\tau}^{t} a(r) d r} d \tau
\end{gathered}
$$

la cual corresponde a la ecuación (C.17) del Lema de Bellman-Gronwall. Si b(t) es constante, entonces $\dot{b}(t)=0$ y la ecuación (C.28) se reduce a:

$$
y(t) \leq b(0) e^{\int_{0}^{\prime} a(r) d r}
$$

que corresponde a la ecuación (C.18) del Lema. 


\section{APÉNDICE D. Vectores, matrices y mapas.}

\section{(Aparecen en los Capitulos 3, 4 y 5)}

\section{Vectores $y$ matrices}

db $M:=$ matriz $M$ diagonal por bloques

$\operatorname{diag} M:=$ matriz $M$ diagonal por bloques

$$
\begin{aligned}
& A_{u / o}=\Gamma_{u / o}-K_{u / o} \Delta_{u / o}, \quad A_{o}^{e}=\Gamma_{o}^{e}-K_{o}^{e} \Delta_{o}^{e} \\
& \Gamma_{u / o}=b d\left[\Gamma_{1}^{u / o}, \ldots, \Gamma_{p / m}^{u / o}\right], \quad \Gamma_{i}^{u / o}{ }_{\left(v_{i} \times v_{i}\right) /\left(\kappa_{i} \times \kappa_{i}\right)}=\left[\begin{array}{ccccc}
0 & 1 & 0 & \ldots & 0 \\
0 & \ldots & & 0 & 1 \\
0 & & \ldots & 0 & 1 \\
0 & & & \ldots & 0
\end{array}\right] \\
& \Pi_{u / o}=b d\left[\pi_{1}^{u / o}, \ldots, \pi_{p / m}^{u / o}\right], \quad \pi_{i}^{u / o}{ }_{\left(v_{i} \times 1\right) /\left(\kappa_{i} \times 1\right)}=\left[\begin{array}{llll}
0 & \ldots & 0 & 1
\end{array}\right]^{\prime} \\
& \Delta_{u / o}=b d\left[\delta_{1}^{u / o}, \ldots, \delta_{p / m}^{u / o}\right], \quad \delta_{i}^{u / o}{ }_{\left(1 \times v_{i}\right) /\left(1 \times \kappa_{i}\right)}=\left[\begin{array}{llll}
1 & 0 & \ldots & 0
\end{array}\right] \\
& \Gamma_{o}^{e}=b d\left[\Gamma_{1}^{e}, \ldots, \Gamma_{m}^{e}\right], \quad \Gamma_{i}^{e}=\Gamma_{i}^{o} \\
& \Pi_{o}^{e}=b d\left[\pi_{1}^{e}, \ldots, \pi_{m}^{e}\right], \quad \pi_{i}^{e}=\left[\begin{array}{ll}
\pi_{i}^{o} & 0
\end{array}\right] \\
& \Delta_{o}^{e}=b d\left[\delta_{1}^{e}, \ldots, \delta_{m}^{e}\right], \quad \delta_{i}^{e}=\left[\begin{array}{ll}
\delta_{i}^{o} & 0
\end{array}\right] \\
& K_{o}^{e}=b d\left[\left(s_{o} k_{11}^{o}, \ldots, s_{o}^{\kappa_{1}} k_{\kappa_{1}}^{o}, s_{o}^{x_{1}+1} k_{1}^{I}\right)^{\prime}, \ldots,\left(s_{o} k_{1 m}^{o}, \ldots, s_{o}^{\kappa_{m}} k_{k_{m} m}^{o}, s_{o}^{\kappa_{m}+1} k_{m}^{I}\right)^{\prime}\right] \\
& e_{I}=\left[e_{1}^{I}, \ldots, e_{\kappa_{1}}^{I} ; \ldots ; e_{\kappa_{m}-1}^{I}, \ldots, e_{\kappa}^{I}\right]^{\prime}, \quad e_{q}=\left[e_{1}^{q}, \ldots, e_{m}^{q}\right]^{\prime} \\
& A_{c}=\Gamma_{c}+\Pi_{c} K_{c} \\
& \Gamma_{c}=b d\left[\Gamma_{1}^{c}, \ldots, \Gamma_{m}^{c}\right], \quad \Gamma_{i\left(\kappa_{i}^{c} \times \kappa_{i}^{c}\right)}^{c}=\left[\begin{array}{ccccc}
0 & 1 & 0 & \ldots & 0 \\
0 & \ldots & & 0 & 1 \\
0 & & \ldots & 0 & 1 \\
0 & & & \ldots & 0
\end{array}\right] \\
& \Pi_{c}=b d\left[\pi_{1}^{c}, \ldots, \pi_{m}^{c}\right], \quad \pi_{i\left(\kappa_{i}^{c} \times 1\right)}^{c}=\left[\begin{array}{llll}
0 & \ldots & 0 & 1
\end{array}\right]^{\prime} \\
& \Delta_{c}=b d\left[\delta_{1}^{c}, \ldots, \delta_{m}^{c}\right], \quad \delta_{i\left(1 \times \kappa_{i}^{c}\right)}^{c}=\left[\begin{array}{llll}
1 & 0 & \ldots & 0
\end{array}\right]
\end{aligned}
$$




\section{Mapas no lineales}

$$
\begin{aligned}
& z=\left[z_{u}^{\prime}, z_{I}^{\prime}, z_{I I}^{\prime}\right]^{\prime} \\
& \varphi\left(z_{I}, z_{I I}, z_{u}, v, b\right)=\left\{\varphi\left(x, x_{u}, \dot{x}_{u}, b\right)\right\}_{\left\{\left(x^{\prime}, x_{u}^{\prime}\right)^{\prime}=\phi^{-1}[z, b], x_{u}=\Gamma_{u} z_{u}+\Pi_{u} v\right.} \\
& \theta\left[z_{I}, z_{I I}, z_{u}, v, b\right]=\left\{\left(\frac{\partial \phi_{I I}}{\partial x}\right) f+\left(\frac{\partial \phi_{I I}}{\partial u}\right) \dot{x}_{u}\right\}_{\left\{\left(x^{\prime}, x_{u}^{\prime}\right)^{\prime}=\phi^{-1}[z, b], \dot{x}_{u}=\Gamma_{u} z_{u}+\Pi_{u^{\prime}} v\right.} \\
& \omega\left[e_{I I}, t\right]=\left\{\theta\left[z_{u}, z_{l}, z_{I I}+e_{I I}, v, b\right]-\theta\left[z_{I}, z_{I I}, z_{u}, v, b\right]\right\}_{[\mathrm{z}(\mathrm{t}), v(\mathrm{t})]} \\
& q_{I}\left(e_{u}, e_{I}, e_{I l}, v, e_{b}, t\right)=\left\{\varphi\left(z_{I}+e_{I}, z_{I I}+e_{I I}, z_{u}+e_{u}, v, b+e_{b}\right)-\varphi\left(z_{I}, z_{I I}, z_{u}, v, b\right)\right\}_{[\mathrm{z}(\mathrm{t}), v(t)]} \\
& q_{I I}\left(e_{u}, e_{l}, e_{I I}, v, e_{b}, t\right)=\left\{\theta\left(z_{I}+e_{I}, z_{I I}+e_{I I}, z_{u}+e_{u}, v, b+e_{b}\right)-\theta\left(z_{I}, z_{I I}+e_{I I}, z_{u}, v, b\right)\right\}_{[\mathrm{z}(\mathrm{t}), v(\mathrm{t})]} \\
& q_{r}\left(e_{I}, e_{u}, v, t\right)=q_{I}\left(e_{u}, e_{I}, e_{I I}, v, e_{b}, t\right)-q_{I}\left(0,0, e_{I I}, 0, e_{b}, t\right) \\
& q_{l}\left(e_{I I}, e_{b}, t\right)=q_{I}\left(0,0, e_{I I}, 0, e_{b}, t\right) \\
& z_{c}=\left[z_{I}^{c^{\prime}}, z_{I I}^{c^{\prime}}\right]^{\prime} \\
& \theta_{c}\left[z_{I}, z_{I I}, K_{c}\left(z_{I}-\bar{z}_{I}\right), x_{d}, b\right]=\left\{\left(\frac{\partial \phi_{I I}}{\partial x}\right) f+\left(\frac{\partial \phi_{I I}}{\partial x_{d}}\right) \dot{x}_{d}\right\}_{\left\{x=\phi^{-1}[z, b], \dot{x}_{d}=\Gamma_{d l} z_{d}+\Pi_{d} v\right\}} \\
& \omega_{c}\left[e_{I I}^{c}, t\right]=\left\{\theta\left[z_{I}^{c}, z_{I I}^{c}, \bar{v}+K_{c} e_{I}^{c}, x_{d}, b\right]-\theta\left[z_{I}^{c}, z_{I I}^{c}+e_{I I}^{c}, \bar{v}+K_{c} e_{I}^{c}, x_{d}, b\right]\right\}_{\left[\mathrm{z}(\mathrm{t}), x_{d}(\mathrm{t})\right]} \\
& q_{I I}^{c}\left(e_{I}^{c}, e_{I I}^{c}, e_{d}, t\right)=\left\{\theta_{c}\left(z_{I}^{c}, z_{I I}^{c}+e_{I I}^{c}, \bar{v}+K_{c} e_{I}^{c}, x_{d}, b\right)-\theta_{c}\left(z_{I}^{c}+e_{I}^{c}, z_{I I}^{c}+e_{I I}^{c}, \bar{v}, \bar{x}_{d}, b\right)\right\}_{\left[\mathrm{z}(\mathrm{t}), x_{d}(\mathrm{t})\right]}
\end{aligned}
$$




\section{APÉNDICE E. Prueba de los Teoremas y Corolarios.}

\section{Prueba del Teorema 2 (Capitulo 3)}

Primero analicemos las dinámicas del error de estimación exo-observable, observable, y no observable (3.39) de manera independiente, y luego la interacción entre ellas.

\section{Acotamiento del error del exo-observador}

Tomemos la dinámica de $e_{\mathrm{u}}$ (3.39):

$$
\dot{e}_{u}=A_{u} e_{u}-\Pi_{u} \mathrm{v}(t), \quad\|\mathrm{v}(t)\| \leq \varepsilon_{v}
$$

e integremos

$$
e_{u}(t)=e^{A_{u}\left(t-t_{o}\right)} e_{u o}-\int_{t_{o}}^{t} e^{A_{u}(t-\tau)} \Pi_{u} v(t) d \tau
$$

donde $A_{\mathrm{u}}$ es estable y cumple con la desigualdad (3.46). Tomando normas en la ecuación (E.2), y sustituyendo la desigualdad de acota a $v(t)(\mathrm{E} .1)$ y a la exponencial de $A_{\mathrm{u}} t(3.47)$ se llega a:

$$
\left\|e_{u}(t)\right\| \leq a_{u}\left\|e_{u o}\right\| e^{-s_{u} \lambda_{u}\left(t-t_{o}\right)}+a_{u} \varepsilon_{v} \int_{t_{o}}^{t} e^{-s_{u} \lambda_{u}(t-\tau)} d \tau
$$

donde:

$$
\int_{t_{o}}^{t} e^{-s_{u} \lambda_{u}(t-\tau)} d \tau=\frac{1}{s_{u} \lambda_{u}}\left[1-e^{-s_{u} \lambda_{u}\left(t-t_{o}\right)}\right] \leq \frac{1}{l_{u}}, \quad l_{u}:=s_{u} \lambda_{u}
$$

Entonces, sustituyendo (E.4) en (E.3):

$$
\left\|e_{u}(t)\right\| \leq a_{u}\left\|e_{u o}\right\| e^{-l_{u}\left(t-t_{o}\right)}+\frac{a_{u}}{l_{u}} \varepsilon_{v}
$$

$\underline{\text { Acotamiento del error de la parte observable del estimador }}$

Tomemos la dinámica de $e_{\mathrm{I}}$ (3.39):

$$
\dot{e}_{I}=A_{o} e_{I}+\Pi_{o} q_{I}\left(e_{I}, e_{I I}, e_{u}, e_{b}, t\right)
$$

e integremos 


$$
e_{I}(t)=e^{A_{o}\left(t-t_{o}\right)} e_{I o}+\int_{t_{o}}^{t} e^{A_{o}(t-\tau)} \Pi_{o} q_{I}\left(e_{I}, e_{I I}, e_{u}, e_{b}, t\right) d \tau
$$

donde $A_{\mathrm{o}}$ es estable y cumple con la desigualdad (3.48). Tomando normas en la ecuación (E.7), y sustituyendo la desigualdad que acota a la exponencial de $A_{\mathrm{o}} t$ (3.48) se obtiene

$$
\left\|e_{I}(t)\right\| \leq a_{o}\left\|e_{I o}\right\| e^{-s_{o} \lambda_{o}\left(t-t_{o}\right)}+a_{o} \int_{t_{o}}^{t} e^{-s_{o} \lambda_{o}(t-\tau)}\left\|q_{I}\left(e_{I}, e_{I I}, e_{u}, e_{b}, \tau\right)\right\| d \tau
$$

y sustituyendo ahora la desigualdad que acota a la perturbación $q_{1}(3.42)$

$$
\begin{aligned}
\left\|e_{I}(t)\right\| \leq & a_{o}\left\|e_{I o}\right\| e^{-s_{o} \lambda_{o}\left(t-t_{o}\right)}+a_{o} M_{I} \int_{t_{o}}^{t} e^{-s_{o} \lambda_{o}(t-\tau)}\left\|e_{I}(\tau)\right\| d \tau+ \\
& a_{o} \int_{t_{o}}^{t} e^{-s_{o} \lambda_{o}(t-\tau)}\left[M_{I I}\left\|e_{I I}(\tau)\right\|+M_{u}\left\|e_{u}(\tau)\right\|+M_{b}\left\|e_{b}\right\|\right] d \tau
\end{aligned}
$$

donde $\left\|e_{b}\right\| \leq \varepsilon_{b}$ (3.38). Apliquemos el siguiente cambio de coordenadas

$$
\theta(t)=e^{s_{o} \lambda_{o} t}\left\|e_{I}(t)\right\|
$$

de tal forma que la desigualdad (E.9) obtenga la siguiente forma (cuya estructura corresponde a la desigualdad de Bellman-Gronwall, ver Apéndice $\mathrm{C}$ ):

$$
\theta(t) \leq a_{o} \theta\left(t_{o}\right)+a_{o} M_{I} \int_{t_{o}}^{t} \theta(\tau) d \tau+a_{o} \int_{t_{o}}^{t} e^{s_{o} \lambda_{o} \tau}\left[M_{I I}\left\|e_{I I}(\tau)\right\|+M_{u}\left\|e_{u}(\tau)\right\|+M_{b} \varepsilon_{b}\right] d \tau
$$

Definiendo las variables $\alpha$ y $\beta$ como

$$
\begin{aligned}
& \alpha \quad=a_{o} M_{I} \\
& \beta(t)=a_{o} \theta\left(t_{o}\right)+a_{o} \int_{t_{o}}^{t} e^{s_{o} \lambda_{o} \tau}\left[M_{I I}\left\|e_{I I}(\tau)\right\|+M_{u}\left\|e_{u}(\tau)\right\|+M_{b} \varepsilon_{b}\right] d \tau
\end{aligned}
$$

entonces (E.11) se vuelve

$$
\theta(t) \leq \alpha \int_{t_{o}}^{t} \theta(\tau) d \tau+\beta(t)
$$

Empleando la desigualdad de Bellman-Gronwall (lema en Apéndice C), se llega a:

$$
\theta(t) \leq \beta\left(t_{o}\right) e^{a_{o} M_{/}\left(t-t_{o}\right)}+\int_{t_{o}}^{t} \dot{\beta}(\tau) e^{a_{o} M_{l}(t-\tau)} d \tau
$$

donde

$$
\beta\left(t_{o}\right)=a_{o} \theta\left(t_{o}\right), \quad \dot{\beta}(\tau)=a_{o} e^{s_{o} \lambda_{o} \tau}\left[M_{I I}\left\|e_{I I}(\tau)\right\|+M_{u}\left\|e_{u}(\tau)\right\|+M_{b} \varepsilon_{b}\right]
$$

Sustituyendo $\beta$ y su derivada (E.15) en la desigualdad (E.14)

$$
\theta(t) \leq a_{o} e^{a_{o} M_{t}\left(t-t_{o}\right)} \theta\left(t_{o}\right)+a_{o} e^{a_{o} M_{t} t} \int_{t_{o}}^{t} a_{o} e^{\left(s_{o} \lambda_{o}-a_{o} M_{t}\right) \tau}\left[M_{I I}\left\|e_{I I}(\tau)\right\|+M_{u}\left\|e_{u}(\tau)\right\|+M_{b} \varepsilon_{b}\right] d \tau
$$

o bien 


$$
\begin{gathered}
\theta(t) \leq a_{o} e^{a_{o} M_{I}\left(t-t_{o}\right)} \theta\left(t_{o}\right)+a_{o} e^{a_{o} M_{I} t} \int_{t_{o}}^{t} e^{\left(s_{o} \lambda_{o}-a_{o} M_{I}\right) \tau}\left[M_{I I}\left\|e_{I I}(\tau)\right\|+M_{u}\left\|e_{u}(\tau)\right\|\right] d \tau+ \\
\frac{a_{o} M_{b}}{s_{o} \lambda_{o}-a_{o} M_{I}}\left[e^{s_{o} \lambda_{o} t}-e^{s_{o} \lambda_{o} \tau+a_{o} M_{I}\left(t-t_{o}\right)}\right] \varepsilon_{b}
\end{gathered}
$$

Regresando está última expresión a las variables originales (E.10) se obtiene:

$$
\begin{aligned}
\left\|e_{I}(t)\right\| \leq & a_{o}\left\|e_{I o}(t)\right\| e^{-\left(s_{o} \lambda_{o}-a_{o} M_{I}\right)\left(t-t_{o}\right)}+a_{o} \int_{t_{o}}^{t} e^{-\left(s_{o} \lambda_{o}-a_{o} M_{I}\right)(t-\tau)}\left[M_{I I}\left\|e_{I I}(\tau)\right\|+M_{u}\left\|e_{u}(\tau)\right\|\right] d \tau+ \\
& \frac{a_{o} M_{b}}{s_{o} \lambda_{o}-a_{o} M_{I}}\left[1-e^{-\left(s_{o} \lambda_{o} \tau-a_{o} M_{I}\right)\left(t-t_{o}\right)}\right] \varepsilon_{b}
\end{aligned}
$$

en donde el termino $\left[1-e^{-\left(s_{o} \lambda_{o} \tau-a_{o} M_{i}\right)\left(t-t_{o}\right)}\right] \leq 1$, y definiendo el factor de decaimiento $L_{\mathrm{l}} \mathrm{de}$ la parte observable como

$$
L_{I}:=s_{o} \lambda_{o}-a_{o} M_{I}
$$

entonces sustituyendo estas dos consideraciones en (E.17), se obtiene finalmente el acotamiento del error observable:

$$
\left\|e_{I}(t)\right\| \leq a_{o}\left\|e_{I o}(t)\right\| e^{-L_{I}\left(t-t_{o}\right)}+a_{o} \int_{t_{o}}^{t} e^{-L_{I}(t-\tau)}\left[M_{I I}\left\|e_{I I}(\tau)\right\|+M_{u}\left\|e_{u}(\tau)\right\|\right] d \tau+\frac{a_{o} M_{b}}{L_{I}} \varepsilon_{b}
$$

\section{Acotamiento del error de la parte no observable del estimador}

Tomemos la dinámica de $e_{\mathrm{II}}$ (3.39):

$$
\dot{e}_{I I}=\omega\left(e_{I I}, t\right)+q_{I I}\left(e_{I}, e_{I I}, e_{u}, e_{b}, t\right)
$$

Ya que este sistema sin perturbar (i.e. $q_{\mathrm{II}}=0$ )

$$
\dot{e}_{I I}^{*}=\omega\left(e_{I l}^{*}, t\right)
$$

es E-estable, y de acuerdo al Teorema Converso de Lyapunov (ver Teorema C.4 en Apéndice $\mathrm{C})$, entonces existe una función de Lyapunov $V\left(e_{l l}^{*}, t\right)$ con cuatro constantes positivas $\alpha_{i}$ tal que las siguientes desigualdades

$$
\begin{gathered}
\alpha_{1}\left\|e_{I I}^{*}(t)\right\|^{2} \leq V\left(e_{I I}^{*}, t\right) \leq \alpha_{2}\left\|e_{I I}^{*}(t)\right\|^{2} \\
\dot{V}=\left\|\frac{\partial V}{\partial e_{I I}^{*}} \omega\left(e_{I I}^{*}, t\right)+\frac{\partial V}{\partial t}\right\| \leq-\alpha_{3}\left\|e_{I I}^{*}(t)\right\|^{2} \\
\left\|\frac{\partial V}{\partial e_{I I}^{*}}\right\| \leq \alpha_{4}\left\|e_{I I}^{*}(t)\right\|
\end{gathered}
$$

se cumplen en una vecindad de la trayectoria del error no observable $e_{\mathrm{II}}(t)=0$ del sistema (E.21) o (3.40) con $q_{\mathrm{II}}=0$. 
Siguiendo el método directo de Lyapunov, tomemos a la función $V\left(e_{I I}^{*}, t\right)$ del sistema no perturbado como una función de Lyapunov candidato para el sistema perturbado. Entonces, su derivada a lo largo de la trayectoria $e_{\mathrm{II}}(t)$ del sistema perturbado (3.40) está dada por:

$$
\dot{V}=\frac{\partial V}{\partial e_{l I}} \dot{e}_{l I}+\frac{\partial V}{\partial t}
$$

sustituyendo la dinámica de $e_{\mathrm{II}}$ (E.20)

$$
\dot{V}=\frac{\partial V}{\partial e_{l l}} \omega\left(e_{I I}, t\right)+\frac{\partial V}{\partial t}+\frac{\partial V}{\partial e_{I I}} q_{I l}\left(e_{I}, e_{I I}, e_{u}, e_{b}, t\right)
$$

Tomando normas, y sustituyendo las desigualdades (E.23) y (E.24) del Teorema Converso en la ecuación (E.26), se obtiene la desigualdad

$$
\dot{V} \leq \alpha_{3}\left\|e_{I I}(t)\right\|^{2}+\alpha_{4}\left\|e_{I I}(t)\right\|\left\|q_{I I}\left(e_{I}, e_{I I}, e_{u}, e_{b}, t\right)\right\|
$$

De la desigualdad (E.22) tenemos que

$$
\left\|e_{I I}(t)\right\|^{2} \leq \frac{V}{\alpha_{1}}
$$

entonces (E.27) se vuelve

$$
\dot{V} \leq \frac{\alpha_{3}}{\alpha_{1}} V+\alpha_{4}\left\|e_{I I}(t)\right\|\left\|q_{I I}\left(e_{1}, e_{I I}, e_{u}, e_{b}, t\right)\right\|
$$

Si en vez de $V$, utilizamos el cambio de coordenadas $\vartheta$, tenemos que

$$
\vartheta=V^{\frac{1}{2}}, \quad \dot{\vartheta}=\dot{V} / 2 \vartheta
$$

entonces la ecuación (E.29) puede reescribirse como

$$
\dot{\vartheta} \leq-\frac{\alpha_{3}}{2 \alpha_{1}} \vartheta+\frac{\alpha_{4}}{2}\left[\frac{\left\|e_{I I}(t)\right\|^{2}}{V}\right]^{\frac{1}{2}}\left\|q_{I I}\left(e_{I}, e_{I I}, e_{u}, e_{b}, t\right)\right\|
$$

Sustituyendo nuevamente la desigualdad (E.28) en (E.31) tenemos que

$$
\dot{\vartheta} \leq-\frac{\alpha_{3}}{2 \alpha_{1}} \vartheta+\frac{\alpha_{4}}{2 \alpha_{1}^{\frac{1}{2}}}\left\|q_{I l}\left(e_{l}, e_{I I}, e_{u}, e_{b}, t\right)\right\|
$$

e integrando esta desigualdad lineal $\vartheta$, se tiene que

$$
\vartheta(t) \leq e^{-\frac{\alpha_{3}}{2 \sigma_{I}}\left(t-t_{o}\right)} \vartheta\left(t_{o}\right)+\frac{\alpha_{4}}{2 \alpha_{1}^{\frac{1}{2}}} \int_{t_{o}}^{t} e^{-\frac{\alpha_{3}}{2 q_{I}}(t-\tau)}\left\|q_{I I}\left(e_{I}, e_{I I}, e_{u}, e_{b}, t\right)\right\| d \tau
$$

Utilizando las desigualdades (E.22) del Teorema Converso de Lyapunov, el cambio de coordenadas $\vartheta$ (E.30) como función de $V$ y $e_{\mathrm{II}}$ está dado por:

$$
\begin{aligned}
& \vartheta(t)=V(t)^{\frac{1}{2}} \geq \alpha_{1}^{\frac{1}{2}}\left\|e_{I I}(t)\right\| \\
& \vartheta\left(t_{o}\right)=V\left(t_{o}\right)^{\frac{1}{2}} \leq \alpha_{2}^{\frac{1}{2}}\left\|e_{I I o}\right\|
\end{aligned}
$$


Sustituyendo este cambio de coordenadas (E.34), así como la norma de $q_{11}$ [definida en ecuación (3.43)] en la desigualdad (E.33) se llega a:

$$
\left\|e_{I I}(t)\right\| \leq\left(\frac{\alpha_{2}}{\alpha_{1}}\right)^{\frac{1}{2}}\left\|e_{I I o}\right\| e^{-\frac{\alpha_{3}}{2 I_{I}}\left(t-t_{o}\right)}+\frac{\alpha_{4}}{2 \alpha_{1}} \int_{t_{o}}^{t} e^{-\frac{\alpha_{3}}{\alpha_{I}}(t-\tau)}\left[N_{I}\left\|e_{I}(\tau)\right\|+N_{u}\left\|e_{u}(\tau)\right\|+N_{b}\left\|e_{b}\right\|\right] d \tau
$$

Si definimos:

$$
A_{I I}=\max \left[\left(\frac{\alpha_{2}}{\alpha_{1}}\right)^{\frac{1}{2}}, \frac{\alpha_{4}}{2 \alpha_{1}}\right], \quad L_{l l}=\frac{\alpha_{3}}{\alpha_{1}}
$$

Se obtiene finalmente el acotamiento del error no observable:

$$
\left\|e_{I I}(t)\right\| \leq A_{I I}\left\|e_{I I o}\right\| e^{-L_{I I}\left(t-t_{o}\right)}+A_{I I} \int_{t_{o}}^{t} e^{-L_{I I}}\left[N_{I}\left\|e_{I}(\tau)\right\|+N_{u}\left\|e_{u}(\tau)\right\|\right] d \tau+\frac{A_{I I} N_{b}}{L_{I I}} \varepsilon_{b}
$$

\section{$\underline{\text { Acotamiento del error de estimación }}$}

Resumiendo, el sistema dinámico del error de estimación (3.39) satisface los acotamientos (E.5), (E.19), y (E.37)

$$
\begin{aligned}
& \left\|e_{u}(t)\right\| \leq a_{u}\left\|e_{u o}\right\| e^{-L_{u}\left(t-t_{o}\right)}+\frac{a_{u}}{l_{u}} \varepsilon_{v} \\
& \left\|e_{I}(t)\right\| \leq a_{o}\left\|e_{I o}(t)\right\| e^{-L_{I}\left(t-t_{o}\right)}+a_{o} \int_{t_{o}}^{t} e^{-L_{I}(t-\tau)}\left[M_{I I}\left\|e_{I I}(\tau)\right\|+M_{u}\left\|e_{u}(\tau)\right\|\right] d \tau+\frac{a_{o} M_{b}}{L_{I}} \varepsilon_{b} \\
& \left\|e_{I I}(t)\right\| \leq A_{I I}\left\|e_{I I}\right\| e^{-L_{I I}\left(t-t_{o}\right)}+A_{I I} \int_{t_{o}}^{t} e^{-L_{I I}}\left[N_{I}\left\|e_{I}(\tau)\right\|+N_{u}\left\|e_{u}(\tau)\right\|\right] d \tau+\frac{A_{I I} N_{b}}{L_{I I}} \varepsilon_{b}
\end{aligned}
$$

que corresponden a un conjunto de desigualdades integrales. Ahora procederemos a reescribir este conjunto de desigualdades integrales en una forma diferencial equivalente, tomando en cuenta el Teorema de Separación (ver Apéndice C). Para tal efecto, tomemos la versión igualdad, donde los acotamientos de $\left(e_{\mathrm{u}}, e_{\mathrm{I}}, e_{\mathrm{II}}\right)$ (E.38) se reemplazan por su cota superior $\left(\sigma_{\mathrm{u}}, \sigma_{\mathrm{I}}, \sigma_{\mathrm{II}}\right)$ :

$$
\begin{aligned}
& \sigma_{u}(t)=a_{u}\left\|e_{u o}\right\| e^{-l_{u}\left(t-t_{o}\right)}+\frac{a_{u}}{l_{u}} \varepsilon_{v} \\
& \sigma_{I}(t)=a_{o}\left\|e_{l o}(t)\right\| e^{-L_{I}\left(t-t_{o}\right)}+a_{o} \int_{t_{o}}^{t} e^{-L_{I}(t-\tau)}\left[M_{I I}\left\|e_{I I}(\tau)\right\|+M_{u}\left\|e_{u}(\tau)\right\|\right] d \tau+\frac{a_{o} M_{b}}{L_{I}} \varepsilon_{b} \\
& \sigma_{I I}(t)=A_{I I}\left\|e_{I I o}\right\| e^{-L_{I I}\left(t-t_{o}\right)}+A_{I I} \int_{t_{o}}^{t} e^{-L_{I I}}\left[N_{I}\left\|e_{I}(\tau)\right\|+N_{u}\left\|e_{u}(\tau)\right\|\right] d \tau+\frac{A_{I I} N_{b}}{L_{I I}} \varepsilon_{b}
\end{aligned}
$$

y reescribamos este sistema (E.39) en forma diferencial 


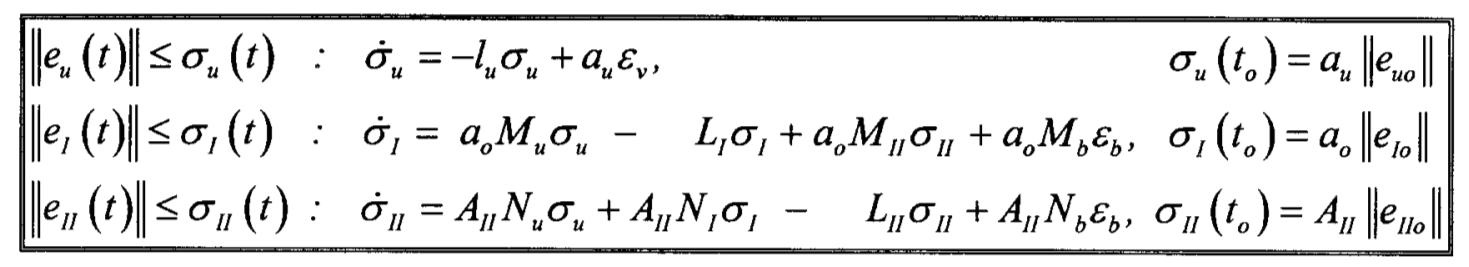

o bien, en notación vectorial

$$
\begin{aligned}
& \begin{array}{ll}
\dot{\sigma}_{u}=-l_{u} \sigma_{u}+a_{u} \varepsilon_{v}, & \sigma_{u}\left(t_{o}\right)=\sigma_{u o} \\
\dot{\sigma}_{a}=A \sigma_{a}+B_{u} \sigma_{u}+B_{b} \varepsilon_{b}, & \sigma_{a}\left(t_{o}\right)=\sigma_{a o}
\end{array} \\
& A=\left[\begin{array}{cc}
-L_{I I} & a_{o} M_{I I} \\
A_{I I} N_{I} & -L_{I}
\end{array}\right], \quad B_{u}=\left[\begin{array}{c}
a_{o} M_{u} \\
A_{I I} N_{u}
\end{array}\right], \quad B_{b}=\left[\begin{array}{l}
a_{o} M_{b} \\
A_{l I} N_{b}
\end{array}\right]
\end{aligned}
$$

Puesto que de la definición (3.38) $e_{u o}=\left(\hat{z}_{u o}-z_{u o}\right)$ y $\left[e_{I o}{ }^{\prime}, e_{I l o}\right]^{\prime}=\left[\left(\hat{z}_{I o}-z_{I o}\right)^{\prime}\right.$, $\left.\left(\hat{z}_{l o}-z_{l o}\right)^{\prime}\right]^{\prime} ;$ y del cambio de coordenadas (3.29) $z_{u}=x_{u} y\left[z_{l}{ }^{\prime}, z_{l I}{ }^{\prime}\right]^{\prime}=\phi\left(x, x_{u}, b\right)$ se tiene que:

$$
\begin{aligned}
& e_{u}=\hat{x}_{u}-x_{u}, \\
& {\left[e_{I o}, e_{I l o}{ }^{\prime}\right]^{\prime}=\phi\left(\hat{x}_{o}, \hat{x}_{u o}, \beta\right)-\phi\left(x_{o}, x_{u o}, b\right)}
\end{aligned}
$$

Tomando normas en (E.43), y sustituyendo el acotamiento de $\phi\left(x, x_{u}, b\right)(3.27)$, se tiene que

$$
\begin{aligned}
& \left\|e_{u o}\right\|=\left\|\hat{x}_{u o}-x_{u o}\right\|, \\
& {\left[\left\|e_{I o}\right\|^{\prime},\left\|e_{I I o}\right\|^{\prime}\right]^{\prime} \leq D_{x}\left\|\hat{x}_{o}-x_{o}\right\|+D_{u}\left\|\hat{x}_{u o}-x_{u o}\right\|+D_{b} \varepsilon_{b}}
\end{aligned}
$$

Por lo tanto las condiciones iniciales $\sigma_{u o}$ y $\sigma_{a o}$ definida por (E.40)-(E.41),

$$
\begin{aligned}
& \sigma_{u o}=a_{u}\left\|e_{u o}\right\|, \\
& \sigma_{a o}=\left[\begin{array}{l}
\sigma_{l o} \\
\sigma_{I l o}
\end{array}\right]:=\left[\begin{array}{c}
a_{o}\left\|e_{l o}\right\| \\
A_{I I}\left\|e_{I l o}\right\|
\end{array}\right]
\end{aligned}
$$

equivalen a [sustituyendo (E.44) en (E.45)]

$$
\begin{aligned}
& \sigma_{u o}=a_{u}\left\|\hat{x}_{u o}-x_{u o}\right\| \\
& \sigma_{a o} \leq a_{\sigma}\left[D_{x}\left\|\hat{x}_{o}-x_{o}\right\|+D_{u}\left\|\hat{x}_{u o}-x_{u o}\right\|+D_{b} \varepsilon_{b}\right], \quad a_{\sigma}=\max \left(a_{o}, A_{I I}\right)
\end{aligned}
$$




\section{Criterio de convergencia del error de estimación}

Para que el sistema (E.41) sea estable, y con ello las trayectorias $\left(x_{\mathrm{u}}, x, y\right)$ del estimador sean convergentes, se requiere que la matriz $A$ [definida en (E.42)] sea estable. De acuerdo al criterio de Hurwitz (Boyce y DiPrima, 1995) para que la matriz $A$ sea estable debe cumplirse que

$$
\begin{aligned}
& \operatorname{det}(A)>0 \\
\therefore & L_{I} L_{I I}>a_{0} A_{I I} M_{I I} N_{I}
\end{aligned}
$$

la cual es equivalente a [empleando (E.18)]

$$
s_{o} \lambda_{o}>a_{o} M_{I}+a_{o}\left(\frac{A_{I I}}{L_{I I}}\right) M_{I I} N_{I}
$$

En alguna vecindad alrededor del origen de la dinámica del error, esta expresión se cumple si en $e=0$ se satisface la siguiente desigualdad

$$
s_{o} \lambda_{o}>a_{o} m_{I}+a_{o} c_{x}^{\phi}\left(a_{x} / \lambda_{I I}\right) m_{I I} n_{I}, \quad \kappa<n
$$

donde $a_{I l},\left(a_{x}, \lambda_{I I}\right), \quad\left(m_{F}, m_{I P}, n_{I}\right)$ están definidas por (3.32), (3.34), y (3.45), respectivamente. Esta desigualdad (E.49) constituye la condición de convergencia (Teorema 3.1) para el estimador-P con $\kappa<n$ (esto es, cuando existe una dinámica no observable). En caso contrario, si $\kappa=n$ entonces no existe $e_{\text {II }}$ y la condición de convergencia se reduce a

$$
s_{o} \lambda_{o}>a_{o} m_{l}, \quad \kappa=n
$$




\section{Prueba del Corolario del Teorema 2 (Capitulo 3)}

\section{Caracterización de la convergencia (Alvarez, 2000)}

Empleando la definición del error de estimación (3.38) y las desigualdades en (E.40) que definen las cotas superiores del error de estimación, se obtiene que la trayectoria del error de estimación en las entradas está acotada en coordenadas originales como sigue:

$$
\begin{aligned}
& \left\|e_{u}(t)\right\| \leq \sigma_{u}(t) \\
\Rightarrow \quad & \left\|\hat{z}_{u}(t)-z_{u}(t)\right\| \leq \sigma_{u}(t) \\
\Rightarrow & \left\|\hat{x}_{u}(t)-x_{u}(t)\right\| \leq \sigma_{u}(t)
\end{aligned}
$$

De forma similar, empleando la definición del error de estimación (3.38) y las desigualdades en (E.40), se obtiene que las trayectorias de los errores de estimación observable y no observable están acotadas conjuntamente como sigue:

$$
\left.\begin{array}{c}
\left\|e_{I}(t)\right\| \leq \sigma_{I}(t) \\
\left\|e_{I I}(t)\right\| \leq \sigma_{I I}(t)
\end{array}\right\} \Rightarrow\left\|\phi\left(\hat{x}, \hat{x}_{u}, \beta\right)-\phi\left(x, x_{u}, b\right)\right\| \leq \sigma_{a}(t), \quad \sigma_{a}(t)=\left[\begin{array}{c}
\sigma_{I}(t) \\
\sigma_{I I}(t)
\end{array}\right]
$$

Por otra parte, el error de estimación en los estados $x$, está delimitado por [sustituir la definición de invertibilidad en $x$ (3.26) en la desigualdad (3.28)]:

$$
\|\hat{x}(t)-x(t)\| \leq F_{z}\left\|\phi\left(\hat{x}, \hat{x}_{u}, \beta\right)-\phi\left(x, x_{u}, b\right)\right\|+F_{u}\left\|\hat{x}_{u}-x_{u}\right\|+F_{b}\|\beta-b\|
$$

Incluyendo las normas $\left\|\phi\left(\hat{x}, \hat{x}_{u}, \beta\right)-\phi\left(x, x_{u}, b\right)\right\|$ (E.51) y $\|\hat{x}(t)-x(t)\|$ (E.52) en esta expresiôn (E.53), se obtiene el siguiente acotamiento de la trayectoria del error de estimación en los estados:

$$
\|\hat{x}-x\| \leq F_{z} \sigma_{a}(t)+F_{u} \sigma_{u}(t)+F_{b} \varepsilon_{b}
$$

Empleando la norma del error $\left\|e_{I}(t)\right\|$ (E.40) junto con la definición del error de estimación observable (3.38) se tiene que:

$$
\begin{aligned}
& \left\|e_{I}(t)\right\| \leq \sigma_{I}(t) \\
& \left\|\phi_{I}\left(\hat{x}, \hat{x}_{u}, \beta\right)-\phi_{I}\left(x, x_{u}, b\right)\right\| \leq \sigma_{I}(t)
\end{aligned}
$$

y sustituyendo la ecuación (3.7) que define el mapa observable $\phi_{1}$, se obtiene la trayectoria del error de estimación en las salidas:

$$
\|\hat{y}(t)-y(t)\| \leq \sigma_{I}(t)
$$


En resumen, las trayectorias del error de estimación en las entradas, estados, y salidas de la planta están acotadas por [(E.51), (E.54), (E.55)]:

$$
\begin{array}{|l}
\left\|\hat{x}_{u}(t)-x_{u}(t)\right\| \leq \sigma_{u}(t) \\
\|\hat{x}(t)-x(t)\| \leq F_{z} \sigma_{a}(t)+F_{u} \sigma_{u}(t)+F_{b} \varepsilon_{b} \\
\|\hat{y}(t)-y(t)\| \leq \sigma_{I}(t)
\end{array}
$$

donde $\sigma_{\mathrm{u}}(t)$ y $\sigma_{\mathrm{a}}(t)=\left[\sigma_{\mathrm{I}}(t)^{\prime}, \sigma_{\mathrm{II}}(t)^{\prime}\right]^{\prime}$ están definidos por la solución del sistema dinámico (E.41). Además, la estabilidad de la matriz $A$ (E.42) implica la existencia de un par de números positivos $\left(A_{\mathrm{a}}, L_{\mathrm{a}}\right)$ tal que la siguiente desigualdad se cumple:

$$
\left\|e^{A t}\right\| \leq A_{a} e^{-L_{a} t}, \quad\left(A_{a}, L_{a}\right)=\left(1, L_{l}\right) \text { si } \kappa=n
$$

Para obtener una dependencia explícita de las desigualdades (E.56), integremos sucesivamente las ecuaciones diferenciales (E.41) que definen $\sigma_{\mathrm{u}}(t), \sigma_{\mathrm{I}}(t)$, y $\sigma_{\mathrm{II}}(t)$. Primero integrando para $\sigma_{\mathrm{u}}(t)$, se llega a:

$$
\begin{aligned}
& \dot{\sigma}_{u}=-l_{u} \sigma_{u}+a_{u} \varepsilon_{v}, \quad \sigma_{u}\left(t_{o}\right)=\sigma_{u o} \\
& \sigma_{u}(t)=\sigma_{u o} e^{-l_{u}\left(t-t_{o}\right)}+a_{u} \varepsilon_{v} \int_{t_{o}}^{t} e^{-l_{u}(t-\tau)} d \tau
\end{aligned}
$$

donde [en forma similar a (E.4)]

$$
\int_{t_{o}}^{t} e^{-l_{u}(t-\tau)} d \tau \leq \frac{1}{l_{u}}
$$

Si definimos

$$
g_{u}=\frac{a_{u}}{l_{u}}
$$

entonces, sustituyendo (E.59) y (E.60) en (E.58) se llega a:

$$
\sigma_{u}(t)=\sigma_{u o} e^{-l_{u}\left(t-t_{o}\right)}+g_{u} \varepsilon_{v}
$$

Sustituyendo está ecuación (E.61) en (E.56), se obtiene finalmente la caracterización de la trayectoria del error de estimación en las entradas:

$$
\left\|\hat{x}_{u}(t)-x_{u}(t)\right\| \leq \sigma_{u o} e^{-l_{u}\left(i-t_{o}\right)}+b_{u}, \quad b_{u}:=g_{u} \varepsilon_{v}
$$

donde $\sigma_{\mathrm{uo}}$ está dada por (E.46).

Integrando ahora para $\sigma_{\mathrm{a}}(t)$ [EDO definida en (E.41)]:

$$
\dot{\sigma}_{a}=A \sigma_{a}(t)+B_{u} \sigma_{u}(t)+B_{b} \varepsilon_{b}, \quad \sigma_{a}\left(t_{o}\right)=\sigma_{a o}
$$




$$
\sigma_{a}(t)=e^{A\left(t-t_{o}\right)} \sigma_{a o}+\int_{t_{o}}^{t} e^{A(t-\tau)}\left[B_{u} \sigma_{u}(\tau)+B_{b} \varepsilon_{b}\right] d \tau
$$

Aplicando normas, y sustituyendo la norma para la matriz $A$ (E.57):

$$
\left\|\sigma_{a}(t)\right\| \leq A_{a} \sigma_{a a} e^{-L_{a}\left(t-t_{o}\right)}+A_{a} \int_{t_{o}}^{t} e^{-L_{a}(t-\tau)}\left[\left\|B_{u}\right\| \sigma_{u}(\tau)+\left\|B_{b}\right\| \varepsilon_{b}\right] d \tau
$$

sustituyendo $\sigma_{\mathrm{u}}(t)$ (E.61) en esta última:

$$
\left\|\sigma_{a}(t)\right\| \leq A_{a} \sigma_{a o} e^{-L_{a}\left(t-t_{o}\right)}+A_{a}\left\|B_{u}\right\| \sigma_{u o} \int_{t_{o}}^{t} e^{-L_{a} t+l_{u} t_{o}+\left(L_{a}-l_{u}\right) \tau} d \tau+A_{a}\left(\frac{a_{u}}{l_{u}}\left\|B_{u}\right\| \varepsilon_{v}+\left\|B_{b}\right\| \varepsilon_{b}\right) \int_{t_{o}}^{t} e^{-L_{a}(t-\tau)} d \tau(\mathrm{E} .65)
$$

donde

$$
\begin{gathered}
\int_{t_{o}}^{t} e^{-L_{a} t+l_{u} t_{o}+\left(L_{a}-l_{u}\right) \tau} d \tau \leq \frac{1}{l_{u}-L_{a}} e^{-L_{a}\left(t-t_{o}\right)}, \quad \text { con } l_{u}>L_{a} \\
\int_{t_{o}}^{t} e^{-L_{a}(t-\tau)} d \tau \leq \frac{1}{L_{a}}
\end{gathered}
$$

por lo tanto,

$$
\left\|\sigma_{a}(t)\right\| \leq\left(A_{a} \sigma_{a o}+\frac{A_{a}}{l_{u}-L_{a}}\left\|B_{u}\right\| \sigma_{u o}\right) e^{-L_{a}\left(t-t_{o}\right)}+\frac{A_{a}}{L_{a}}\left(\frac{a_{u}}{l_{u}}\left\|B_{u}\right\| \varepsilon_{v}+\left\|B_{b}\right\| \varepsilon_{b}\right)
$$

Si definimos:

$$
g_{a}=\frac{A_{a}}{L_{a}}, \quad g_{u a}=\frac{A_{a}}{l_{u}-L_{a}}
$$

entonces la ecuación (E.67) se reduce a:

$$
\left\|\sigma_{a}(t)\right\| \leq\left(A_{a} \sigma_{a o}+g_{u a}\left\|B_{u}\right\| \sigma_{u o}\right) e^{-L_{a}\left(t-t_{o}\right)}+g_{a}\left(g_{u}\left\|B_{u}\right\| \varepsilon_{v}+\left\|B_{b}\right\| \varepsilon_{b}\right)
$$

Sustituyendo $\sigma_{\mathrm{u}}(t)$ y $\sigma_{\mathrm{a}}(t)$ [ecuaciones (E.61) y (E.69), respectivamente] en $\|\hat{x}(t)-x(t)\|$ [ecuación (E.56)] se obtiene:

$$
\begin{gathered}
\|\hat{x}(t)-x(t)\| \leq F_{z} A_{a}\left\|\sigma_{a o}\right\| e^{-L_{a}\left(t-t_{o}\right)}+\left[g_{u a}\left\|B_{u}\right\| e^{-L_{a}\left(t-t_{o}\right)}+F_{u} e^{-l_{u}\left(t-t_{o}\right)}\right] \sigma_{u o}+ \\
g_{u}\left(F_{z} g_{a}\left\|B_{u}\right\|+F_{u}\right) \varepsilon_{v}+\left(F_{z} g_{a}\left\|B_{b}\right\|+F_{b}\right) \varepsilon_{b}
\end{gathered}
$$

como $l_{u}>L_{a}$, entonces $e^{-l_{u}\left(t-t_{o}\right)} \leq e^{-L_{a}\left(t-t_{o}\right)}$. Y redefiniendo variables:

$$
\begin{array}{lll}
L_{a}=L_{x}, & c_{\sigma}^{x}=F_{z} A_{a}, & c_{u}^{x}=F_{z} g_{u a}\left\|B_{u}\right\|+F_{u}, \\
b_{x}=b_{u}^{x} \varepsilon_{v}+b_{b}^{x} \varepsilon_{b}, & b_{u}^{x}=g_{u}\left(F_{z} g_{a}\left\|B_{u}\right\|+F_{u}\right), & b_{b}^{x}=F_{z} g_{a}\left\|B_{b}\right\|+F_{b}
\end{array}
$$

entonces se obtiene finalmente la caracterización de la trayectoria del error de estimación en los estados:

$$
\|\hat{x}(t)-x(t)\| \leq\left(c_{\sigma}^{x} \sigma_{a o}+c_{u}^{x} \sigma_{u o}\right) e^{-L_{x}\left(t-t_{o}\right)}+b_{x}
$$


donde $\sigma_{\mathrm{uo}}$ y $\sigma_{\mathrm{ao}}$ están dadas por (E.46).

Ahora, integrando por último para $\sigma_{\mathrm{I}}(t)$ [EDO definida en (E.40)]:

$$
\begin{gathered}
\dot{\sigma}_{I}=a_{o} M_{u} \sigma_{u}(t)-L_{I} \sigma_{I}(t)+a_{o} M_{I I} \sigma_{I I}(t)+a_{o} M_{b} \varepsilon_{b}, \quad \sigma_{I}\left(t_{o}\right)=a_{o}\left\|e_{I o}\right\| \\
\sigma_{I}(t)=e^{-L_{I}\left(t-t_{o}\right)} a_{o}\left\|e_{I o}\right\|+a_{o} \int_{t_{o}}^{t} e^{-L_{I}(t-\tau)}\left[M_{u} \sigma_{u}(\tau)+M_{I I} \sigma_{I I}(\tau)+M_{b} \varepsilon_{b}\right] d \tau
\end{gathered}
$$

Puesto que $\sigma_{l o}:=a_{o}\left\|e_{l o}\right\|$ y $\sigma_{I I}(t) \leq \sigma_{a}(t)$, y sustituyendo a su vez $\sigma_{\mathrm{u}}(t)$ y $\sigma_{\mathrm{a}}(t)[(\mathrm{E} .61) \mathrm{y}$ (E.69)] en (E.73):

$$
\begin{aligned}
\sigma_{I}(t)= & e^{-L_{I}\left(t-t_{o}\right)} \sigma_{I o}+a_{o} M_{u} \sigma_{u o} \int_{t_{o}}^{t} e^{-L_{I}(t-\tau)-l_{u}\left(\tau-t_{o}\right)} d \tau+a_{o} M_{I I}\left(A_{a} \sigma_{a o}+g_{u a}\left\|B_{u}\right\| \sigma_{u o}\right) \int_{t_{o}}^{t} e^{-L_{l}(t-\tau)-L_{a}\left(\tau-t_{o}\right)} d \tau \\
& +a_{o}\left[g_{u}\left(M_{u}+M_{I I} g_{a}\left\|B_{u}\right\|\right) \varepsilon_{v}+\left(M_{I I} g_{a}\left\|B_{b}\right\|+M_{b}\right) \varepsilon_{b}\right] \int_{t_{o}}^{t} e^{-L_{I}(t-\tau)} d \tau
\end{aligned}
$$

donde cada una de las integrales que aparecen en el lado derecho de la desigualdad están acotadas como sigue

$$
\begin{aligned}
& \int_{t_{o}}^{t} e^{-L_{I}(t-\tau)-l_{u}\left(\tau-t_{o}\right)} d \tau \leq \frac{1}{l_{u}-L_{I}} e^{-L_{l}\left(t-t_{o}\right)}, \\
& \int_{t_{o}}^{t} e^{-L_{l}(t-\tau)-L_{a}\left(\tau-t_{o}\right)} d \tau \leq \frac{1}{l_{u}-L_{I}} e^{-L_{l}\left(t-t_{o}\right)}, \quad\left(l_{u}>L_{a}\right) \\
& \int_{t_{o}}^{t} e^{-L_{l}(t-\tau)} d \tau \leq \frac{1}{L_{I}}
\end{aligned}
$$

Sustituyendo (E.75) en (E.74), y considerando que $\sigma_{l o} \leq \sigma_{a o}$ se obtiene la siguiente expresión:

$$
\begin{aligned}
\sigma_{I}(t)= & \left(1+\frac{A_{a} a_{o} M_{I I}}{l_{u}-L_{I}}\right) e^{-L_{I}\left(t-t_{o}\right)} \sigma_{a o}+\frac{a_{o}}{l_{u}-L_{I}}\left(M_{u}+M_{I I} g_{u a}\left\|B_{u}\right\|\right) e^{-L_{I}\left(t-t_{o}\right)} \sigma_{u o} \\
& +\frac{a_{o}}{L_{I}} g_{u}\left(M_{u}+M_{I I} g_{a}\left\|B_{u}\right\|\right) \varepsilon_{v}+\frac{a_{o}}{L_{I}}\left(M_{b}+M_{I I} g_{a}\left\|B_{b}\right\|\right) \varepsilon_{b}
\end{aligned}
$$

Si definimos

$$
\begin{array}{ll}
c_{\sigma}^{y}=1+\frac{A_{a} a_{o} M_{I I}}{l_{u}-L_{I}}, & c_{u}^{y}=\frac{a_{o}}{l_{u}-L_{I}}\left(M_{u}+M_{I I} g_{u a}\left\|B_{u}\right\|\right), \\
b_{y}=b_{v}^{y} \varepsilon_{v}+b_{b}^{y} \varepsilon_{b}, & b_{v}^{y}=\frac{a_{o}}{L_{I}} g_{u}\left(M_{u}+M_{I I} g_{a}\left\|B_{u}\right\|\right), \quad b_{b}^{y}=\frac{a_{o}}{L_{I}}\left(M_{b}+M_{I I} g_{a}\left\|B_{b}\right\|\right)
\end{array}
$$

Entonces (E.76) se reduce a

$$
\sigma_{I}(t)=\left(c_{\sigma}^{y} \sigma_{a o}+c_{u}^{y} \sigma_{u o}\right) e^{-L_{I}\left(t-t_{o}\right)}+b_{y}
$$


Sustituyendo $\sigma_{\mathrm{I}}(t)$ (E.78) en $\|\hat{y}(t)-y(t)\|$ (E.56), se obtiene finalmente la caracterización de la trayectoria del error de estimación en las salidas:

$$
\|\hat{y}(t)-y(t)\| \leq\left(c_{\sigma}^{y} \sigma_{a o}+c_{u}^{y} \sigma_{u o}\right) e^{-L_{l}\left(t-t_{o}\right)}+b_{y}
$$

Con esto se concluye la prueba del corolario del Teorema 2, ya que las expresiones (E.62), (E.72), y (E.79) corresponden a las desigualdades establecidas en dicho corolario.

QED. 


\section{Prueba del Teorema y del Corolario 3 (Capitulo 3)}

Comparando el conjunto de ecuaciones que determinan la dinámica del error de estimación para el estimador-P (3.39):

$$
\begin{aligned}
& \dot{e}_{u}=A_{u} e_{u}-\Pi_{u} \mathrm{v}(t), \\
& \dot{e}_{I}=A_{o} e_{I}+\Pi_{o} q_{I}\left(e_{I}, e_{I I}, e_{u}, \mathrm{v}, e_{b}, t\right), \\
& \dot{e}_{I I}=\omega\left(e_{I I}, t\right)+q_{I I}\left(e_{I}, e_{I I}, e_{u}, e_{b}, t\right)
\end{aligned}
$$

con las correspondientes para el estimador-PI (3.68)

$$
\begin{aligned}
& \dot{e}_{u}=A_{u} e_{u}-\Pi_{u} \mathrm{v}(t) \text {, } \\
& \mu=\Delta_{u} e_{u} \\
& \dot{e}_{e}=A_{o}^{e} e_{e}+\Pi_{o}^{e} q_{I}\left(e_{I}, e_{I I}, e_{u}, e_{b}, t\right), \quad e_{e}=\left(e_{I}{ }^{\prime}, e_{q}{ }^{\prime}\right)^{\prime}, \quad v=\Delta_{o} e_{I} \\
& \dot{e}_{I I}=\omega\left(e_{I I}, t\right)+q_{I I}\left(e_{I}, e_{I I}, e_{u}, e_{b}, t\right)
\end{aligned}
$$

se determina que son equivalentes, con diferencias en $\left(A_{o}, \Pi_{o}\right)$ y $\left(A_{o}^{e}, \Pi_{o}^{e}\right) ; \mathrm{y}$ recordando que dichas matrices estān acotadas como sigue:

$$
\left\|e^{A_{o}\left(s_{o}\right) t}\right\| \leq a_{o} e^{-s_{o} \lambda_{o} t}, \quad\left\|e^{A_{o}^{e}\left(s_{o}\right) t}\right\| \leq a_{o}^{e} e^{-s_{o} \lambda_{o}^{e} t}, \quad\left\|\Pi_{o}^{e}\right\|=\left\|\Pi_{o}\right\|=1
$$

Por lo tanto se concluye que la dinámica del error de estimación (E.80) del estimador-PI es RE-convergente, si la condición (E.49)-(E.50) del Teorema 1 se cumple cuando el par $\left(A_{o}, \lambda_{o}\right)$ se reemplaza por $\left(A_{o}^{e}, \lambda_{o}^{e}\right)$, la cual corresponde a la condición de convergencia (3.80) del Teorema 2.

A partir de este mismo argumento de comparación, se concluye que el estimador-PI converge-RE de acuerdo al conjunto de desigualdades establecidas en el Teorema 1, cuando el conjunto $\left\{a_{o}, \lambda_{o}, A_{a}, L_{x}\right\}$ se reemplaza por $\left\{a_{o}^{e}, \lambda_{o}^{e}, A_{a}^{e}, L_{x}^{e}\right\}$, donde:

$$
\begin{gathered}
A_{e}=\left[\begin{array}{cc}
-L_{I}^{e} & a_{o}^{e} M_{I I} \\
A_{I I} N_{I} & L_{I I}
\end{array}\right] \\
\left\|e^{A_{a}^{e} t}\right\| \leq a_{a}^{e} e^{-L_{a}^{e} t}, \quad L_{x}^{e}=L_{a}^{e}
\end{gathered}
$$




\section{APÉNDICE F. Técnicas de sintonización.}

\section{Ganancias de referencia del exo-observador}

Como se explicó en la sección 3.8.1, para la sintonización del exo-observador se toma como referencia la versión estocástica del exo-sistema (ec. 3.88):

$$
\begin{aligned}
& \dot{x}_{i}^{u}=\Gamma_{i}^{u} x_{i}^{u}+\Pi_{i}^{u} v_{i}(t) \\
& u_{i}=\delta_{i}^{u} x_{i}^{u}+w_{i}(t), \quad 1 \leq i \leq p
\end{aligned}
$$

para el cual el filtro óptimo (Kwakernaak y Sivan, 1972) está dado por ( $\Sigma_{\mathrm{i}}$ es la matriz de covarianza de dimensión $\left.v_{\mathrm{i}} \mathrm{X} \mathrm{v}_{\mathrm{i}}\right)$ :

$$
\begin{array}{ll}
\dot{\hat{x}}_{i}^{u}=\Gamma_{i}^{u} \hat{x}_{i}^{u}+\Pi_{i}^{u} v_{i}(t)+K_{i}^{u}\left(u_{i}-\delta_{i}^{u} \hat{x}_{i}^{u}\right), & K_{i}^{u}=r_{i}^{-1} \Sigma_{i} \delta_{i}^{u \prime} \\
\dot{\Sigma}=\Sigma_{i} \Gamma_{i}^{u}+\Gamma_{i}^{u} \Sigma_{i}+q_{i} \Pi_{i}^{u} \Pi_{i}^{u \prime}-r_{i}^{-1} \Sigma_{i} \delta_{i}^{u \prime} \delta_{i}^{u} \Sigma_{i}, & 1 \leq i \leq p
\end{array}
$$

Cuando la ecuación de Riccati es resuelta en estado estacionario(i.e. $\dot{\Sigma}=0$ ), se obtiene un filtro un filtro óptimo. A partir de este procedimiento es posible calcular: (a) las ganancias de referencia $K_{i}^{u}(1)$ óptimas, (b) la dinámica (característica) del error de salida en función de las intensidades $q_{\mathrm{i}} \mathrm{y} r_{\mathrm{i}}$, y (c) los polos o valores propios caracteristicos $\left(\lambda_{\mathrm{i}}\right)$. Enseguida se presenta el desarrollo para $v_{\mathrm{i}}=1,2, \mathrm{y}$ posteriormente se resumen los resultados para $v_{i}=1, \ldots$ (en general) en la Tabla F.1.

Caso $v_{i}=1$. Para este caso, la planta y su filtro óptimo están dados por $\left(\Gamma_{i}^{u}=[0]\right.$, $\left.\Pi_{i}^{u}=[1], \delta_{i}^{u}=[1]\right):$

$$
\begin{gathered}
\dot{x}_{1}^{u}=v_{1}(t), \quad u_{1}=x_{1}^{u}+w_{1}(t) \\
\dot{\hat{x}}_{i}^{u}=v_{i}(t)+k_{1 i}^{u}\left(u_{i}-\hat{x}_{i}^{u}\right), \quad k_{1 i}^{u}=\sigma_{1} / r_{i}
\end{gathered}
$$

donde la matriz de covarianza es determinada por $\Sigma=[\sigma], \dot{\sigma}=0$ :

$$
q_{i}-\sigma^{2} / r_{i}=0
$$


Despejando $\sigma_{1}$ de (F.5), y sustituyendo en $k_{11}^{u}$ [definida en (F.4)]:

$$
\sigma=r_{i}\left(q_{i} / r_{i}\right)^{\frac{1}{2}} \quad \Rightarrow \quad k_{11}^{u}=\left(q_{i} / r_{i}\right)^{\frac{1}{2}}
$$

Definiendo el error de estimación como la esperanza matemática $(E)$ de la diferencia entre el valor estimador y el valor real de la medición:

$$
\bar{e}=E\left[\hat{x}_{1}^{u}-x_{1}^{u}\right]
$$

La dinámica del error de salida se obtiene restando (F.3) de (F.4):

$$
\dot{\bar{e}}_{i}=-\left(q_{i} / r_{i}\right)^{\frac{1}{2}} \overline{e_{i}}
$$

cuyo valor propio (polo) es:

$$
\lambda_{1}=-\left(q_{i} / r_{i}\right)^{\frac{1}{2}}
$$

Graficando este valor propio en el plano complejo (ver Figura F.1), puede observarse está localizado a una distancia del origen de $d=\left(q_{i} / r_{i}\right)^{\frac{1}{2}}$ y con un ángulo $\alpha=0$ rad.

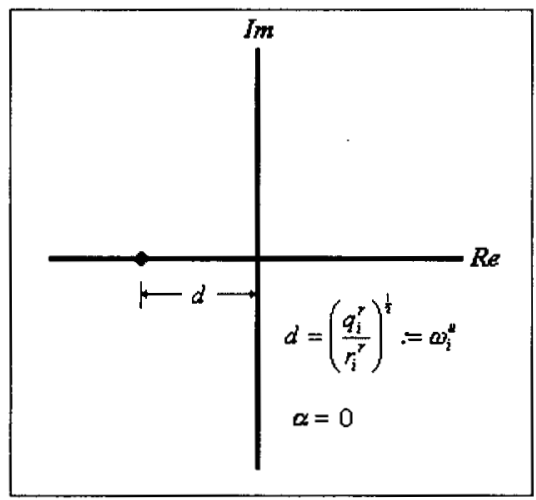

Figura F.1 Localización de polos para el exo-observador, con $v_{\mathrm{i}}=1$ 
Caso $v_{i}=2$. Para este caso, la planta y su filtro óptimo están dados por

$$
\begin{array}{cc}
\dot{x}_{i}^{u}=\Gamma_{i}^{u} x_{i}^{u}+\Pi_{i}^{u} v_{i}(t), & u_{i}=\delta_{i}^{u} x_{i}^{u}+w_{i}(t) \\
\dot{\hat{x}}_{i}^{u}=\Gamma_{i}^{u} \hat{x}_{i}^{u}+\Pi_{i}^{u} v_{i}(t)+K_{i}^{u}\left(u_{i}-\delta_{i}^{u} \hat{x}_{i}^{u}\right), & K_{i}^{u}=r_{i}^{-1} \Sigma_{i} \delta_{i}^{u}, \\
\Gamma_{i}^{u}=\left[\begin{array}{ll}
0 & 1 \\
0 & 0
\end{array}\right], \quad \Pi_{i}^{u}=\left[\begin{array}{l}
0 \\
1
\end{array}\right], \quad \delta_{i}^{u}=\left[\begin{array}{ll}
1 & 0
\end{array}\right], \quad K_{i}^{u}=\left[\begin{array}{c}
k_{1 i}^{u} \\
k_{2 i}^{u}
\end{array}\right], \quad \Sigma_{i}=\left[\begin{array}{ll}
\sigma_{11} & \sigma_{12} \\
\sigma_{21} & \sigma_{22}
\end{array}\right]
\end{array}
$$

donde la matriz de covarianza es determinada por $\dot{\Sigma}=0$ :

$$
\Sigma_{i} \Gamma_{i}^{u}+\Gamma_{i}^{u} \Sigma_{i}+q_{i} \Pi_{i}^{u} \Pi_{i}^{u}{ }^{\prime}-r_{i}^{-1} \Sigma_{i} \delta_{i}^{u}{ }^{\prime} \delta_{i}^{u} \Sigma_{i}=0 \Rightarrow\left\{\begin{array}{l}
\dot{\sigma}_{11}=2 \sigma_{12}-\sigma_{11}^{2} / r_{i} \\
\dot{\sigma}_{12}=\dot{\sigma}_{21}=\sigma_{22}-\sigma_{11} \sigma_{22} / r_{i} \\
\dot{\sigma}_{22}=q_{i}-\sigma_{12}^{2} / r_{i}
\end{array}\right.
$$

Despejando las ganancias óptimas de (F.13), y sustituyendo en $K_{i}^{u}$ [definida en (F.11)]:

$$
\left\{\begin{array} { l } 
{ \sigma _ { 1 1 } = \sqrt { 2 } r _ { i } ( q _ { i } / r _ { i } ) ^ { \frac { 1 } { 4 } } } \\
{ \sigma _ { 1 2 } = \sigma _ { 2 1 } = r _ { i } ( q _ { i } / r _ { i } ) ^ { \frac { 1 } { 2 } } } \\
{ \sigma _ { 2 2 } = \sqrt { 2 } r _ { i } ( q _ { i } / r _ { i } ) ^ { \frac { 3 } { 4 } } }
\end{array} \Rightarrow \quad \left\{\begin{array}{l}
k_{1 i}^{u}=\sqrt{2}\left(q_{i} / r_{i}\right)^{\frac{1}{4}} \\
k_{2 i}^{u}=\left(q_{i} / r_{i}\right)^{\frac{1}{2}}
\end{array}\right.\right.
$$

La dinámica del error de salida se obtiene restando (F.10) de (F.11):

$$
\ddot{\overline{e_{i}}}+\sqrt{2}\left(q_{i} / r_{i}\right)^{\frac{1}{4}} \dot{\bar{e}}_{i}+\left(q_{i} / r_{i}\right)^{\frac{1}{2}} \overline{e_{i}}=0
$$

cuyos valores propios son:

$$
\lambda_{1,2}=-(1 / \sqrt{2})\left(q_{1} / r_{1}\right)^{\frac{1}{4}} \pm(1 / \sqrt{2})\left(q_{1} / r_{1}\right)^{\frac{1}{4}} j
$$

La localización de estos valores propios en el plano complejo (ver Figura F.2) son a una distancia del origen de $d=\left(q_{1} / r_{1}\right)^{\frac{1}{4}}$ y con un ángulo $\alpha= \pm \pi / 4 \mathrm{rad}$. 


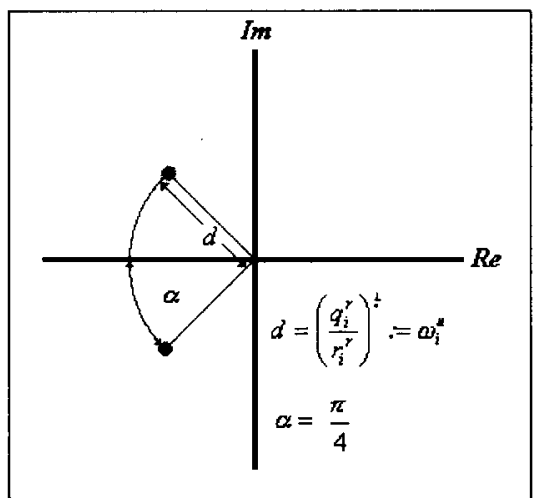

Figura F.2 Localización de polos para el exo-observador, con $v_{\mathrm{i}}=2$

Caso $v_{i}$ en general. Realizando el procedimiento descrito anteriormente para $v_{j}=1$, $2, \ldots$ se obtienen los resultados tabulados en tabla de abajo. En el último renglón se han incluido expresiones generales para cualquier caso de $v_{\mathrm{i}}$. Cabe señalar que la forma sistemática de la localización de los polos (ver Figura 3.3, y su explicación correspondiente en la sección 3.7.2 del Capitulo 3), permite generar fórmulas recursivas para obtener distancias y ángulos para la asignación de polos, y a partir de éstos determinar las coordenadas de los polos $\left(\lambda_{\mathrm{i}}\right)$, y finalmente con éstos se puede obtener el polinomio característico. Este polinomio característico con $s^{k}=\bar{e}^{(k)}$ (i.e. $s^{0}=\bar{e}, s^{1}=\dot{\bar{e}}, s^{2}=\ddot{\bar{e}}, \ldots$ ) corresponde a la dinámica del error de salida, y cuyos coeficientes corresponden a las ganancias del filtro óptimo, las cuales están en función de $\left(q_{i} / r_{i}\right): K_{i}^{u}\left(q_{i} / r_{i}\right)=\left[k_{1 i}^{u}, \ldots, k_{v_{i}}^{u}\right]$.

Fijando el cociente de referencia $\left(q_{i}^{r} / r_{i}^{r}\right)$ (ruido del ruido del modelo/ruido de medición), y definiendo:

$$
\omega_{i}^{u}=\left(q_{i}^{r} / r_{i}^{r}\right)^{\frac{1}{2 v_{i}}}, \quad s_{u}=\left(q_{i} / r_{i}\right)^{\frac{1}{2 v_{i}} / \omega_{i}^{u}}
$$

tal que la ganancia $K_{i}^{u}\left(s_{u}\right)$ corresponde al i-ésimo vector de la matriz parametrizada $K_{u}\left(s_{u}\right)$ :

$$
K_{u}\left(s_{u}\right)=d b\left[\left(s_{u} k_{11}^{u}, \ldots, s_{u}^{v_{1}} k_{v_{1} 1}^{u}\right)^{\prime}, \ldots,\left(s_{u} k_{1 p}^{u}, \ldots, s_{u}^{v_{p}} k_{v_{p} p}^{u}\right)^{\prime}\right], \quad s_{u}>0
$$

obteniéndose que las ganancias de referencias $K_{i}^{u}(1)=\left[k_{1 i}^{u}, \ldots, k_{v_{i}}^{u}\right]$ están determinadas por $\omega_{i}^{u}$, y dadas en la Tabla 3.1 (Capitulo 3). 
Tabla F.1 Resumen de ganancias óptimas y localización de polos para el exo-observador

\begin{tabular}{|c|c|c|c|c|}
\hline \multirow{2}{*}{$\begin{array}{c}\text { orden } \\
v_{i}\end{array}$} & \multirow{2}{*}{$\begin{array}{c}\text { Ganancias } \\
K_{i}^{u}=\left[k_{i}^{u}, \ldots, k_{w i}^{u}\right]\end{array}$} & \multicolumn{3}{|c|}{ Polos } \\
\hline & & $\lambda_{i}\left(i=1, \ldots, v_{i}\right)$ & $d$ (distancia) & $\alpha(\mathrm{rad})$ \\
\hline 1 & $k_{11}^{u}=\left(q_{1} / r_{1}\right)^{\frac{1}{2}}$ & $\lambda_{1}=-\left(q_{i} / r_{i}\right)^{\frac{1}{2}}$ & $\left(q_{i} / r_{i}\right)^{\frac{1}{2}}$ & 0 \\
\hline \multirow{2}{*}{2} & $k_{1 i}^{u}=1.412\left(q_{i} / r_{i}\right)^{\frac{1}{4}}$ & $\lambda_{1}=(-0.707+0.707 j)\left(q_{1} / r_{1}\right)^{\frac{1}{4}}$ & \multirow{2}{*}{$\left(q_{i} / r_{i}\right)^{\frac{1}{4}}$} & $\pi / 4$ \\
\hline & $k_{2 i}^{u}=\left(q_{i} / r_{i}\right)^{\frac{1}{2}}$ & $\lambda_{2}=(-0.707-0.707 j)\left(q_{1} / r_{1}\right)^{\frac{1}{4}}$ & & $-\pi / 4$ \\
\hline \multirow{3}{*}{3} & $k_{1 i}^{u}=2\left(q_{i} / r_{i}\right)^{\frac{1}{6}}$ & $\lambda_{1}=-\left(q_{i} / r_{i}\right)^{\frac{1}{6}}$ & \multirow{3}{*}{$\left(q_{i} / r_{i}\right)^{\frac{1}{6}}$} & 0 \\
\hline & $k_{2 i}^{u}=2\left(q_{i} / r_{i}\right)^{\frac{1}{3}}$ & $\lambda_{2}=(-0.5+0.866 j)\left(q_{1} / r_{1}\right)^{\frac{1}{6}}$ & & $\pi / 3$ \\
\hline & $k_{3 i}^{u}=\left(q_{i} / r_{i}\right)^{\frac{1}{2}}$ & $\lambda_{3}=(-0.5-0.866 j)\left(q_{1} / r_{1}\right)^{\frac{1}{6}}$ & & $-\pi / 3$ \\
\hline \multirow{4}{*}{4} & $k_{1 i}^{u}=2.613\left(q_{i} / r_{i}\right)^{\frac{1}{3}}$ & $\lambda_{1}=(-0.924+0.383 j)\left(q_{1} / r_{1}\right)^{\frac{1}{s}}$ & \multirow{4}{*}{$\left(q_{i} / r_{t}\right)^{\frac{1}{8}}$} & $\pi / 8$ \\
\hline & $k_{2 i}^{u}=3.414\left(q_{i} / r_{i}\right)^{\frac{1}{4}}$ & $\lambda_{2}=(-0.924-0.383 j)\left(q_{1} / r_{1}\right)^{\frac{1}{8}}$ & & $-\pi / 8$ \\
\hline & $k_{3 i}^{u}=2.613\left(q_{i} / r_{i}\right)^{\frac{3}{8}}$ & $\lambda_{3}=(-0.383+0.924 j)\left(q_{1} / r_{1}\right)^{\frac{1}{8}}$ & & $3 \pi / 8$ \\
\hline & $k_{4 i}^{u}=\left(q_{i} / r_{i}\right)^{\frac{1}{2}}$ & $\lambda_{4}=(-0.383-0.924 j)\left(q_{1} / r_{1}\right)^{\frac{1}{8}}$ & & $-3 \pi / 8$ \\
\hline \multirow{2}{*}{$\begin{array}{c}v_{\mathrm{i}} \\
\text { (general) }\end{array}$} & $\begin{array}{l}k_{1,}^{u}, \ldots, k_{v i}^{u} \text { son los } \\
\text { coeficientes del } \\
\text { polinomio: }\end{array}$ & \multirow{2}{*}{$\begin{array}{l}\lambda_{l}=-d \cos (\alpha) \pm d \operatorname{sen}(\alpha) j \\
l=0,1, \ldots, v_{i}\end{array}$} & \multirow[t]{2}{*}{$\left(q_{i} / r_{i}\right)^{\frac{1}{2 \eta_{i}}}$} & $\begin{array}{l}\text { si } v_{i}: \text { impar } \Rightarrow \\
\alpha= \pm l \pi / v_{i} \\
l=0,1, \ldots,\left(v_{i}-1\right) / 2\end{array}$ \\
\hline & $\begin{array}{l}\prod_{l=1}^{v !}\left(s-\lambda_{l}\right)=0 \Rightarrow \\
s^{v_{l}}+k_{1, i}^{u} s^{v_{i-1}-1}+\ldots+k_{v_{i}}^{u}=0\end{array}$ & & & $\begin{array}{l}\text { si } \quad v_{i}: \text { par } \Rightarrow \\
\pm\left(l+\frac{1}{2}\right) \pi / v_{i} \\
l=0,1, \ldots, v_{i} / 2-1\end{array}$ \\
\hline
\end{tabular}




\section{Configuración de Butterworth}

En el siguiente teorema (Kwakernaak y Sivan, 1972) se establecen las propiedades asintóticas de un filtro/observador óptimo (invariante en tiempo) en estado estacionario. Observándose que la configuración que se establece para estos sistemas, llamada configuración de Butterworth, corresponde exactamente a la localización de polos obtenida en la sección anterior.

Teorema 1 (Configuración de Butterworth). Considere el sistema n-dimensional invariante en tiempo

$$
\begin{aligned}
& \dot{x}(t)=A x(t)+B u(t)+g \omega_{3}(t), \\
& \eta(t)=c x(t)+\omega_{2}(t)
\end{aligned}
$$

donde $\omega_{3}$ es un ruido blanco escalar con intensidad $V_{3}, \omega_{2}$ es un ruido blanco escalar no correlacionado con $\omega_{3}$ y con intensidad $V_{2}, g$ es un vector columna, y $c$ es un vector renglón. Suponer que $\{A, g\}$ es estabilizable y $\{A, c\}$ detectable. Sea $H(s)$ la función de transferencia escalar

$$
H(s)=c(s I-A)^{-1} g=\frac{\psi(s)}{\phi(s)}=\frac{\alpha \prod_{i=1}^{p}\left(s-v_{i}\right)}{\prod_{i=1}^{n}\left(s-\pi_{i}\right)}
$$

donde $\phi(s)$ es el polinomio caracteristico de el sistema, y $\pi_{\mathrm{i}}, \mathrm{i}=1,2, \ldots, \mathrm{n}$, son sus valores característicos. Entonces los valores característicos del observador óptimo en estado estacionario son los ceros (localizados en la mitad izquierda del plano) del polinomio

$$
(-1)^{n} \phi(s) \phi(-s)\left[1+\frac{V_{3}}{V_{2}} H(-s) H(s)\right]
$$

Como resultado, las siguientes aseveraciones se cumplen:

(a) Cuando $V_{2} / V_{3} \rightarrow 0, p$ de los $n$ polos del observador óptimo en estado estacionario tienden a los números $\hat{v}_{i}, \mathrm{i}=1,2, \ldots, p$, donde

$$
\hat{v}_{i}=\left\{\begin{array}{lll}
v_{i} & \text { si } & \operatorname{Re}\left(v_{i}\right) \leq 0 \\
-v_{i} & \text { si } & \operatorname{Re}\left(v_{i}\right)>0
\end{array}\right.
$$

(b) Cuando $V_{2} / V_{3} \rightarrow 0$, los $n$ - $p$ polos del observador óptimo tienden asintóticamente a líneas rectas, las cuales se interceptan en el origen y hacen ángulos con el eje real negativo de 


$$
\begin{aligned}
& \pm l \frac{\pi}{n-p}, \quad l=0,1, \ldots, \frac{n-p-1}{2} \quad \text { si } n-p \text { es impar } \\
& \pm \frac{\left(l+\frac{1}{2}\right) \pi}{n-p}, \quad l=0,1, \ldots, \frac{n-p}{2}-1 \quad \text { si } n-p \text { es par }
\end{aligned}
$$

Estos polos se encuentran (equidistantes) a una distancia desde el origen de

$$
\omega_{o}=\left(\alpha^{2} \frac{V_{3}}{V_{2}}\right)^{\frac{1}{2(n-p)}}
$$

(c) Cuando $V_{2} / V_{3} \rightarrow \infty, \operatorname{los} n$ polos del observador tienden a los números $\hat{\pi}_{i}, \mathrm{i}=1,2$, $\ldots, n$, donde

$$
\hat{\pi}_{i}=\left\{\begin{array}{lll}
\pi_{i} & \text { si } & \operatorname{Re}\left(\pi_{i}\right) \leq 0 \\
-\pi_{i} & \text { si } & \operatorname{Re}\left(\pi_{i}\right)>0
\end{array} .\right.
$$

Los polos equidistantes descritos en (b) de este último teorema, corresponden a la configuración de Butterworth. Por otra parte, de acuerdo a este teorema cuando $V_{2} / V_{3} \rightarrow O$ (incisos a y b), el polinomio característico $\phi(s)$ [en (F.20)] puede reescribirse como

$$
\phi(s) \simeq \prod_{i=1}^{p}\left(s-\hat{v}_{i}\right) \prod_{i=1}^{n-p}\left(s-\eta_{i} \omega_{o}\right)
$$

donde los polos $\hat{v}_{i}(\mathrm{i}=1,2, \ldots, p)$ están definidos por $(\mathrm{F} .22), \operatorname{los}$ polos $\eta_{\mathrm{i}}(\mathrm{i}=1,2, \ldots, n$ - $p$ ) constituyen una configuración de Butterworth de orden $n-p$ y radio $1, \mathrm{y} \omega_{0}$ está dado por (F.24). Sustituyendo (F.26) en (F.20), y rearreglando se obtiene la siguiente aproximación

$$
H(s) \simeq \frac{1}{\prod_{i=1}^{n-p}\left(-\frac{s}{\eta_{i} \omega_{o}}+1\right)} \prod_{i=1}^{p}\left(\frac{-\frac{s}{v_{i}}+1}{-\frac{s}{\hat{v}_{i}}+1}\right)
$$

Esta última puede reescribirse como

$$
H(s)=\frac{1}{\chi_{n-p}\left(\frac{s}{\omega_{o}}\right)} \prod_{i=1}^{p}\left(\frac{-\frac{s}{v_{i}}+1}{-\frac{s}{\hat{v}_{i}}+1}\right)
$$

donde $\chi_{n-p}(s)$ es un polinomio de Butterworth de orden $n$ - $p$, que está definido por 


$$
\chi_{n-p}(s)=\prod_{i=1}^{n-p}\left(-\frac{s}{\eta_{i}}+1\right)
$$

Algunos de los polinomios de Butterwoth de bajo orden se listan en la Tabla F.2. La expresión (F.28) muestra que, si la función de transferencia tiene ceros $v_{i}$ estrictamente en el lado izquierdo del plano complejo, entonces cuando $V_{2} / V_{3} \rightarrow 0$, la función de transferencia (F.28) se aproxima a

$$
H(s) \simeq \frac{1}{\chi_{n-p}\left(\frac{s}{\omega_{o}}\right)}
$$

la cual es llamada Función de Transferencia de Butterworth de orden $n-p$.

Tabla F.2 Polinomios de Butterworth (Kwakernaak y Sivan, 1972)

\begin{tabular}{|c|c|}
\hline Orden & Polinomio caracteristico \\
\hline 1 & $s+1$ \\
\hline 2 & $s^{2}+1.414 s+1$ \\
\hline 3 & $s^{3}+2 s^{2}+2 s+1$ \\
\hline 4 & $s^{4}+2.613 s^{3}+3.414 s^{2}+2.613 s+1$ \\
\hline 5 & $s^{5}+3.236 s^{4}+5.236 s^{3}+5.236 s^{2}+3.236 s+1$ \\
\hline
\end{tabular}




\section{Criterio de error ITAE}

(Stephanopoulos , 1984; Ogata, 1980)

Los criterios de error se fundamentan en integrales de alguna función o función pesada del error de salida del sistema en si. Los criterios más usados son:

1. ISE (Integral of the Square Error), donde

$$
I S E=\int_{0}^{\infty} e^{2}(t) d t
$$

2. IAE (Integral of the Absolute value of the Error), donde

$$
I A E=\int_{0}^{\infty}|e(t)| d t
$$

3. ITAE (Integral of the Time-weighted Absolute of the Error), donde

$$
I T A E=\int_{0}^{\infty} t|e(t)| d t
$$

Donde $e(t)=x(t)-\hat{x}(t)$ es el error de desviación entre la salida deseada y real $(\hat{x}(t)$ y $x(t)$, respectivamente). El criterio de error debe seleccionarse de acuerdo a las características y requerimientos del sistema, y luego ajustar los parámetros (a sus valores óptimos) de tal forma que minimice el ISE, IAE, o ITAE de la respuesta del sistema. Algunas caracteristicas son las siguientes: (a) para eliminar errores grandes, el criterio ISE es mejor que el IAE, ya que los errores están al cuadrado y por lo tanto contribuyen más al valor integral; (b) para eliminar errores pequeños, el criterio IAE es mejor que ISE, ya que al elevar al cuadrado números pequeños $(<1)$ el valor se hace aún más pequeño; y (c) para eliminar errores que persisten por tiempos largos, el criterio ITAE es el más adecuado ya que la presencia de tiempos grandes amplifica el efecto de errores (incluso pequeños) en el valor integral.

De acuerdo a lo anterior, el criterio ITAE es el más adecuado para el caso de la sintonización del exo-observador (i.e. un filtro), cuyo objetivo principal es la eliminación del ruido (persistente en tiempo) en las entradas medidas. Este criterio ha sido aplicado al siguiente sistema de $n$-ésimo orden: 


$$
\frac{C(s)}{R(s)}=\frac{a_{n}}{s^{n}+a_{1} s^{n-1}+\cdots+a_{n-1} s+a_{n}}
$$

y se han determinado los coeficientes óptimos (ver Tabla F.3) que minimizan el error integral (6.33).

Tabla F.3 Función de transferencia basada en el criterio ITAE (Ogata, 1980)

\begin{tabular}{|c|c|}
\hline orden & $R(s)=s^{n}+a_{1} s^{n-1}+\cdots+a_{n+1} s+a_{n}, \quad a_{n}=c_{n}^{n}$ \\
\hline 1 & $s+\omega_{n}$ \\
\hline 2 & $\mathrm{~s}^{2}+1.4 \omega_{n} \mathrm{~s}+\omega_{n}^{2}$ \\
\hline 3 & $\mathrm{~s}^{3}+1.75 \omega_{n} \mathrm{~s}^{2}+2.15 \omega_{n}^{2} \mathrm{~s}+\omega_{n}^{3}$ \\
\hline 4 & $\mathrm{~s}^{4}+2.1 \omega_{n} \mathrm{~s}^{3}+3.4 \omega_{n}^{2} \mathrm{~s}^{2}+2.7 \omega_{n}^{3} \mathrm{~s}+\omega_{n}^{4}$ \\
\hline 5 & $\mathrm{~s}^{5}+2.8 \omega_{n} \mathrm{~s}^{4}+5.0 \omega_{n}^{2} \mathrm{~s}^{3}+5.5 \omega_{n}^{3} \mathrm{~s}^{2}+3.4 \omega_{n}^{4} \mathrm{~s}+\omega_{n}^{5}$ \\
\hline 6 & $\begin{aligned} \mathrm{s}^{6}+3.25 \omega_{n} \mathrm{~s}^{5}+6.60 \omega_{n}^{2} \mathrm{~s}^{4}+ & 8.60 \omega_{n}^{3} \mathrm{~s}^{3}+7.45 \omega_{n}^{4} \mathrm{~s}^{2}+3.95 \omega_{n}^{5} \mathrm{~s} \\
+ & \omega_{n}^{6}\end{aligned}$ \\
\hline
\end{tabular}




\section{Ganancias de referencia del estimador}

Como se explicó en la sección 3.8.2, para la sintonización del estimador se toma como referencia la dinámica LPNA del i-ésimo error de salida [ $v_{i}$ en ec. 3.46 o ec. 3.74 , reescrita de la forma:

$$
v_{i}^{\left(n_{i}\right)}+k_{1}^{i} v_{i}^{\left(n_{i}-1\right)}+\ldots+k_{n_{i}}^{i} v_{i}=0, \quad \begin{cases}n_{i}=\kappa_{i} & \text { estimador - P } \\ n_{i}=\kappa_{i}+1 & \text { estimador - PI }\end{cases}
$$

cuyo polinomio caracteristico

$$
\left(\gamma_{i}\right)^{n_{i}}+k_{1}^{i}\left(\gamma_{i}\right)^{n_{i}-1}+\ldots+k_{n_{i}}^{i}=\prod_{l=1}^{n_{i}}\left(\gamma_{i}-\gamma_{l}^{i}\right)=0
$$

tiene $n_{\mathrm{i}}$ polos de referencia caracteristicos $\left(\gamma_{1}^{\mathrm{i}}, \ldots, \gamma_{\mathrm{n}_{\mathrm{i}}}^{\mathrm{i}}\right)$, los cuales se determinan la matriz de ganancias $K_{i}^{o}(1)=\left[k_{1}^{i}, \ldots, k_{n_{i}}^{i}\right]$. La asignación de polos que aquí se propone se hace con base al análisis de la respuesta transitoria para sistemas lineales de primer y segundo orden (Stephanopoulos, 1984).

Un sistema lineal de primer orden de la forma

$$
a_{1} \dot{y}+a_{o} y=b f(t)
$$

donde $f(t)$ es una función entrada (variante en tiempo), puede reescribirse en la forma estándar (si $a_{o} \neq 0$ )

$$
\tau \dot{y}+y=K_{p} f(t)
$$

donde $\tau=a_{1} / a_{o}$ y $K_{p}=b / a_{o}$ son la constante de tiempo y la ganancia estática del sistema, respectivamente. El polinomio caracteristico de este sistema (F.38) determina la ubicación del único polo existente (F.39) y definido por:

$$
\begin{aligned}
& \tau \gamma+1=0 \\
& \gamma_{1}=-1 / \tau
\end{aligned}
$$

Si $f(t)$ es un cambio escalón, $f(t)=A$, la respuesta transitoria está dada por

$$
y(t)=A K_{p}\left(1-e^{-\frac{t}{t}}\right)
$$

La respuesta exponencial se muestra en la Figura F.3. Aqui puede verse que : (a) la velocidad de convergencia al estado estacionario dependen de la ubicación del único polo real (F.39), esto es, entre menor sea $\tau$, mayor es $\gamma_{1}$, y por tanto mayor velocidad de convergencia; (b) en un tiempo equivalente a una constante de tiempo $\tau$, la curva de respuesta alcanza aproximadamente el $63.2 \%$ de su valor en estado estacionario; y (c) la respuesta queda dentro del $2 \%$ de su valor final en un tiempo $t>4 \tau$. 


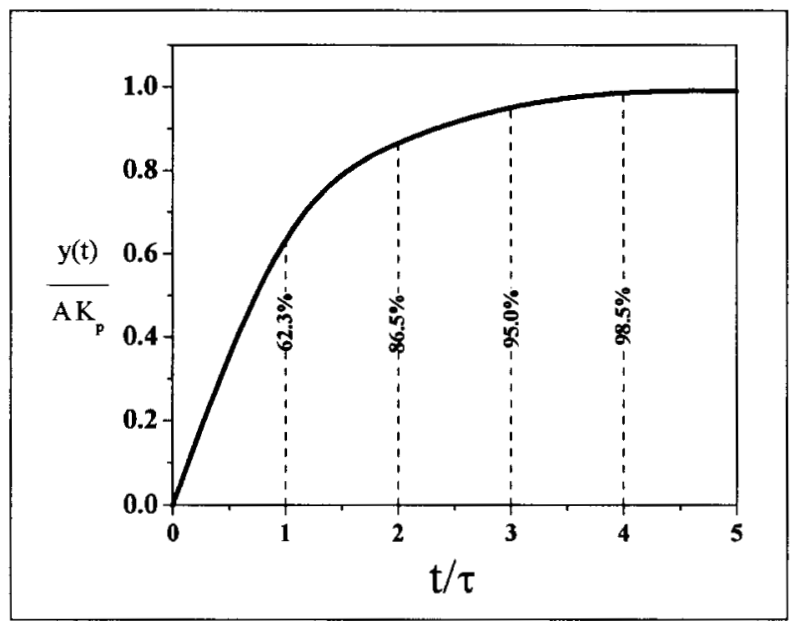

Figura F.3 Respuesta transitoria al escalón, $f(t)=A$, en sistemas de primer orden

Un sistema lineal de segundo orden de la forma

$$
a_{2} \ddot{y}+a_{1} \dot{y}+a_{o} y=b f(t)
$$

puede reescribirse en la forma eständar ( $\mathrm{si} a_{0} \neq 0$ )

$$
\tau^{2} \ddot{y}+2 \xi \tau \dot{y}+y=K_{p} f(t)
$$

donde $\tau=\left(a_{2} / a_{o}\right)^{\frac{1}{2}}, \xi=a_{1} /\left(2 \tau a_{o}\right)$, y $K_{p}=b / a_{o}$ son el periodo natural de oscilación, el factor de amortiguamiento, la ganancia estática del sistema, respectivamente. El polinomio caracteristico de este sistema

$$
\tau^{2} \gamma^{2}+2 \xi \tau \gamma+1=\left(\gamma-\gamma_{1}\right)\left(\gamma-\gamma_{2}\right)=0
$$

determina la ubicación de sus dos polos:

$$
\gamma_{1}, \gamma_{2}=-\frac{1}{\tau}\left(\xi \pm \sqrt{\xi^{2}-1}\right)
$$

Por lo que el comportamiento de la respuesta transitoria $y(t)$ dependerá de la ubicación de los polos (F.44) en el plano complejo, y la localización de estos a su vez queda en función del valor de $\xi$. Distinguiéndose tres casos de comportamiento para sistemas de segundo orden (ver Figura F.4):

1. Si $\xi>1$ entonces los polos son reales y distintos. La respuesta obtenida es sobreamortiguada: no oscilatoria pero lenta.

2. Si $\xi=1$ entonces los polos son reales e iguales. La respuesta obtenida es criticamente amortiguada: no oscilatoria $y$ más rápida que el caso sobreamortiguado. 
3. Si $\xi<1$ entonces los polos son complejos conjugados. La respuesta obtenida es subamortiguada: al inicio es más rápida que los casos 1 y 2 (principalmente con valores de $\xi$ cercanos a 1), y en general es oscilatoria (más pronunciado con valores pequeños de $\xi$ ).

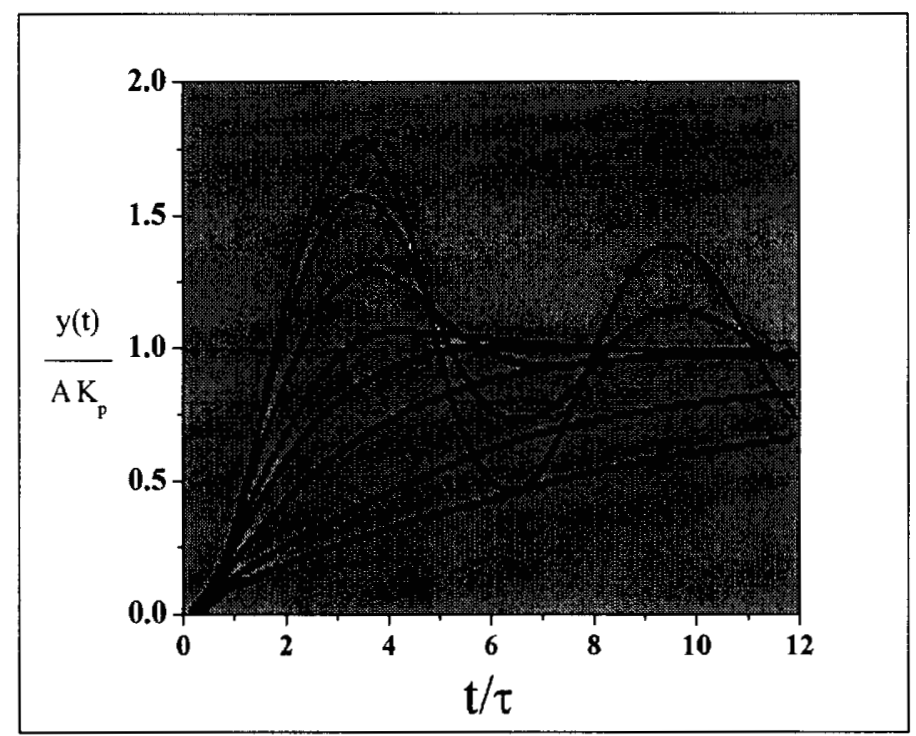

Figura F.4 Respuesta transitoria al escalón, $f(t)=A$, en sistemas de segundo orden

Como puede verse, el hecho de que los polos $\left(\gamma_{1}, \gamma_{2}\right)$ queden en el semiplano izquierdo del lado complejo no garantiza caracteristicas satisfactorias de respuesta transitoria. Si los polos complejos conjugados quedan cercanos al eje imaginario $(\xi \ll 1)$, la respuesta puede presentar excesivas oscilaciones o puede ser muy lenta. Por lo tanto para garantizar características de respuesta rápida y bien amortiguada, es necesario que los polos del sistema queden en una zona específica del plano complejo. En la literatura (Ogata, 1980) se ha reportado $\xi=0.4$ como valor critico (minimo recomendable). 


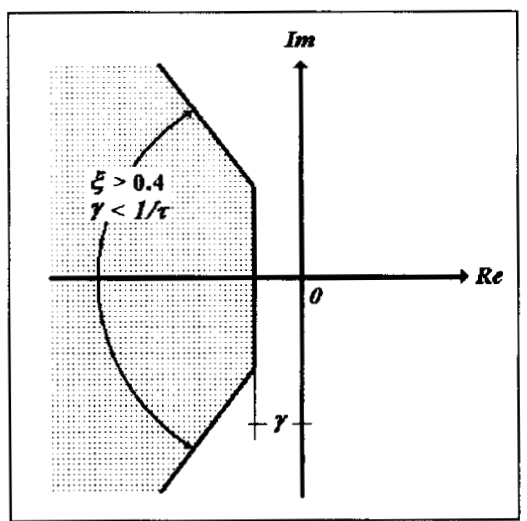

Figura F.5 Región recomendada para la localización de polos (Ogata, 1980)

Tomando en cuentas las consideraciones para sistemas lineales de primer y segundo orden, a continuación se presenta el desarrollo para la asignación de polos para la dinámica LNPA del error de salida (F.34), para indices $n_{\mathrm{i}}=1,2,3$; y luego se resumen los resultados para $n_{\mathrm{i}}=1,2, \ldots$ (en general) en la Tabla F.4.

Caso $n_{i}=1$. Para este caso la dinámica de referencia del error de salida y su polinomio característico correspondientes son

$$
\begin{gathered}
\dot{v}_{i}+k_{1}^{i} v_{i}=0 \\
\gamma_{i}+k_{1}^{i}=\gamma_{i}-\gamma_{1}^{i}=0
\end{gathered}
$$

De (F.46) se obtiene que la ganancia de referencia está dada por

$$
k_{1}^{i}=-\gamma_{1}^{i}
$$

Como se expuso anteriormente, un sistema de primer orden tiene un polo (F.39) definido por (ver Figura F.6):

$$
\gamma_{1}^{i}=-\omega_{i}^{o}, \quad \omega_{i}^{o}:=1 / \tau_{i}
$$

Sustituyendo (F.48) en (F.47) se obtiene la ganancia de referencia en términos de la frecuencia caracteristica $\omega_{i}^{o}$ :

$$
k_{1}^{i}=\omega_{i}^{o}
$$

Caso $n_{i}=2$. La dinámica de referencia del error de salida y su polinomio caracteristico correspondientes son

$$
\begin{gathered}
\ddot{v}_{i}+k_{1}^{i} \dot{v}_{i}+k_{2}^{i} v_{i}=0 \\
\left(\gamma_{i}\right)^{2}+k_{1}^{i} \gamma_{i}+k_{2}^{i}=\left(\gamma_{i}-\gamma_{1}^{i}\right)\left(\gamma_{i}-\gamma_{2}^{i}\right)=0
\end{gathered}
$$

De (F.51) se obtiene que las ganancias de referencia están dadas por 


$$
\begin{aligned}
& k_{1}^{i}=-\left(\gamma_{1}^{i}+\gamma_{2}^{i}\right) \\
& k_{2}^{i}=\gamma_{1}^{i} \gamma_{2}^{i}
\end{aligned}
$$

Para un sistema de segundo orden subamortiguado, sus polos (F.44) están definidos por (ver Figura F.6):

$$
\gamma_{1}^{i}, \gamma_{2}^{i}=-\omega_{i}^{o}\left(\xi_{i} \pm \sqrt{1-\xi_{i}^{2}} j\right), \quad \omega_{i}^{o}:=1 / \tau_{i}, \quad 0.71 \leq \xi \leq 1
$$

La localización de los polos en el plano complejo se muestra en la 0 , donde se ha empleado $\xi_{\mathrm{i}}=0.71$ para obtener un número de condición (i.e., la razón entre el módulo del polo más rápido entre el módulo del polo más lento) igual a uno, con la finalidad de amortiguar/atenuar el efecto de la perturbación $q_{\mathrm{I}}$ en la dinámica del error observable [ec. 3.39 o 3.68 en Capítulo 3]. En general se recomienda seleccionar un factor de amortiguamiento suficientemente grande: $\xi_{\mathrm{i}} \geq 0.71$.

Sustituyendo (F.53) en (F.52) se obtienen las ganancias de referencia en términos de la frecuencia característica $\omega_{i}^{o}$ y del factor de amortiguamiento $\xi_{\mathrm{i}}$ :

$$
\begin{aligned}
& k_{1}^{i}=2 \xi \omega_{i}^{o} \\
& k_{2}^{i}=\left(\omega_{i}^{o}\right)^{2}
\end{aligned}
$$

Caso $n_{i}=3$. La dinámica de referencia del error de salida y su polinomio caracteristico correspondientes son

$$
\begin{gathered}
\dddot{v}_{i}+k_{1}^{i} \ddot{v}_{i}+k_{2}^{i} \dot{v}_{i}+k_{3}^{i} v_{i}=0 \\
\left(\gamma_{i}\right)^{3}+k_{1}^{i}\left(\gamma_{i}\right)^{2}+k_{2}^{i} \gamma_{i}+k_{3}^{i}=\left(\gamma_{i}-\gamma_{1}^{i}\right)\left(\gamma_{i}-\gamma_{2}^{i}\right)\left(\gamma_{i}-\gamma_{3}^{i}\right)=0
\end{gathered}
$$

De (F.56) se obtiene que las ganancias de referencia están dadas por

$$
\begin{aligned}
& k_{1}^{i}=-\left(\gamma_{1}^{i}+\gamma_{2}^{i}+\gamma_{3}^{i}\right) \\
& k_{2}^{i}=\gamma_{1}^{i} \gamma_{2}^{i}+\gamma_{2}^{i} \gamma_{3}^{i}+\gamma_{1}^{i} \gamma_{3}^{i} \\
& k_{3}^{i}=-\gamma_{1}^{i} \gamma_{2}^{i} \gamma_{3}^{i}
\end{aligned}
$$

Para un sistema de tercer orden tomamos la combinación de uno de segundo orden subamortiguado con uno de primer orden, por lo tanto sus polos (F.39)+(F.44) están definidos por (ver Figura F.6):

$$
\left.\begin{array}{l}
\gamma_{1}^{i}, \gamma_{2}^{i}=-\omega_{i}^{o}\left(\xi_{i} \pm \sqrt{1-\xi_{i}^{2}} j\right) \\
\gamma_{3}^{i}=-\omega_{i}^{o}
\end{array}\right\} \quad \omega_{i}^{o}:=1 / \tau_{i}, \quad 0.71 \leq \xi \leq 1
$$

Sustituyendo (F.58) en (F.57) se obtienen las ganancias de referencia en términos de la frecuencia caracteristica $\omega_{i}^{o}$ y del factor de amortiguamiento $\xi_{i}$ : 


$$
\begin{aligned}
& k_{1}^{i}=\left(2 \xi_{i}+1\right) \omega_{i}^{o} \\
& k_{2}^{i}=\left(2 \xi_{i}+1\right)\left(\omega_{i}^{o}\right)^{2} \\
& k_{3}^{i}=\left(\omega_{i}^{o}\right)^{3}
\end{aligned}
$$
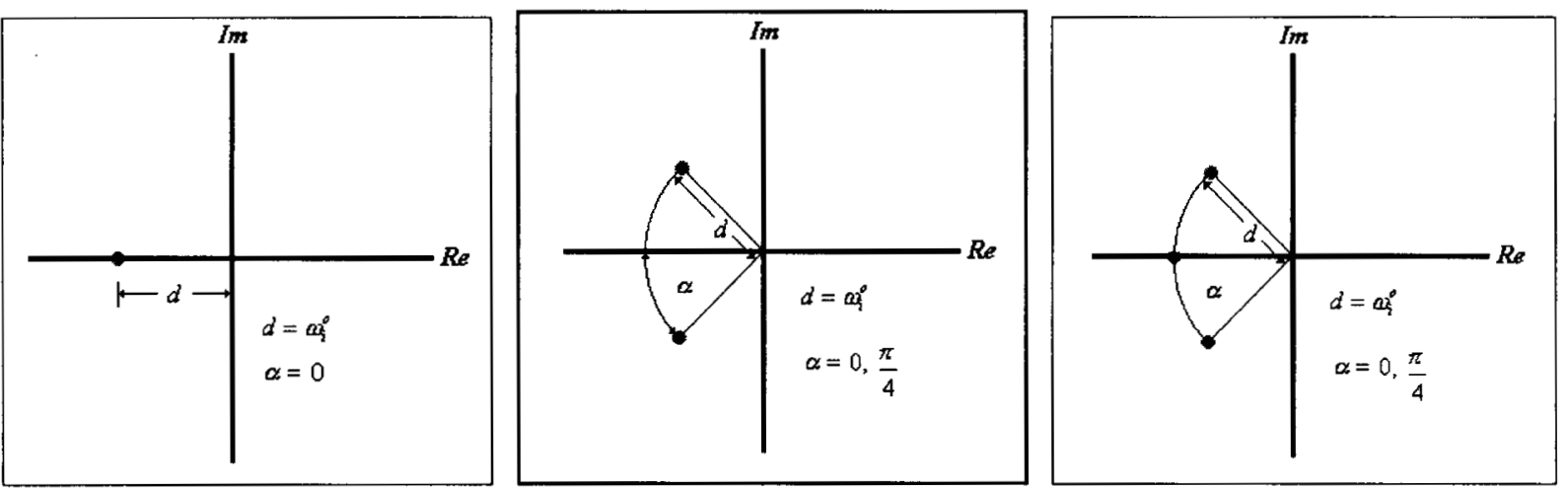

Figura F.6 Localización de polos para el estimador, con $n_{\mathrm{i}}=1,2,3, \mathrm{y} \xi_{\mathrm{i}}=0.71$.

Caso $n_{i}$ en general. Repitiendo el anterior procedimiento se obtienen las ganancias de referencia listadas en la Tabla F.4 (ver también Tabla 3.2 y Figura 3.4). 
Tabla F.4 Resumen de ganancias óptimas y localización de polos para el estimador

\begin{tabular}{|c|c|c|c|c|}
\hline 1 & $k_{\mathrm{I}}^{i}=\omega_{i}^{o}$ & $\gamma_{1}^{i}=-\omega_{i}^{o}$ & $\omega_{i}^{o}$ & 0 \\
\hline \multirow{2}{*}{2} & $k_{1}^{i}=2 \xi \omega_{i}^{o}$ & $\gamma_{1}^{\prime}=-\omega_{i}^{o}\left(\xi_{i}+\sqrt{1-\xi_{i}^{2}} j\right)$ & \multirow[t]{2}{*}{$\omega_{i}^{o}$} & $\operatorname{arctg}\left(-\sqrt{1-\xi_{i}^{2}} / \xi\right)$ \\
\hline & $k_{2}^{i}=\left(\omega_{i}^{o}\right)^{2}$ & $\gamma_{2}^{i}=-\omega_{i}^{o}\left(\xi_{i}-\sqrt{1-\xi_{i}^{2}} j\right)$ & & $\operatorname{arctg}\left(\sqrt{1-\xi_{1}^{2}} / \xi\right)$ \\
\hline \multirow{3}{*}{3} & $k_{1}^{i}=(2 \xi+1) \omega_{i}^{o}$ & $\gamma_{1}^{i}=-\omega_{i}^{o}\left(\xi_{i}+\sqrt{1-\xi_{i}^{2}} j\right)$ & \multirow{3}{*}{$\omega_{i}^{o}$} & 0 \\
\hline & $k_{2}^{i}=(2 \xi+1)\left(\omega_{i}^{o}\right)^{2}$ & $\gamma_{2}^{i}=-\omega_{i}^{o}\left(\xi_{i}-\sqrt{1-\xi_{i}^{2}} j\right)$ & & $\operatorname{arctg}\left(-\sqrt{1-\xi_{i}^{2}} / \xi\right)$ \\
\hline & $k_{3}^{i}=\left(\omega_{i}^{o}\right)^{3}$ & $\gamma_{3}^{i}=-\omega_{i}^{o}$ & & $\operatorname{arctg}\left(\sqrt{1-\xi_{i}^{2}} / \xi\right)$ \\
\hline \multirow{4}{*}{4} & $k_{1}^{i}=4 \xi_{i} \omega_{i}^{o}$ & $\gamma_{1}^{i}=-\omega_{i}^{o}\left(\xi_{i}+\sqrt{1-\xi_{i}^{2}} j\right)$ & \multirow{4}{*}{$\omega_{i}^{o}$} & $\operatorname{arctg}\left(-\sqrt{1-\xi_{i}^{2}} / \xi\right)$ \\
\hline & $k_{2}^{i}=\left(4 \xi_{i}^{2}+1\right)\left(\omega_{i}^{o}\right)^{2}$ & $\gamma_{2}^{i}=-\omega_{i}^{o}\left(\xi_{i}-\sqrt{1-\xi_{i}^{2}} j\right)$ & & $\operatorname{arctg}\left(\sqrt{1-\xi_{i}^{2}} / \xi\right)$ \\
\hline & $k_{3}^{i}=4 \xi_{i}\left(\omega_{i}^{o}\right)^{3}$ & $\gamma_{3}^{i}=-\omega_{i}^{o}\left(\xi_{i}+\sqrt{1-\xi_{i}^{2}} j\right)$ & & $\operatorname{arctg}\left(-\sqrt{1-\xi_{1}^{2}} / \xi\right)$ \\
\hline & $k_{4}^{i}=\left(\omega_{i}^{o}\right)^{4}$ & $\gamma_{4}^{i}=-\omega_{i}^{o}\left(\xi_{i}-\sqrt{1-\xi_{i}^{2}} j\right)$ & & $\operatorname{arctg}\left(\sqrt{1-\xi_{i}^{2}} / \xi\right)$ \\
\hline \multirow[t]{2}{*}{$\begin{array}{c}n_{\mathrm{i}} \\
\text { (general) }\end{array}$} & $\begin{array}{l}{\left[k_{1}^{i}, \ldots, k_{n_{i}}^{i}\right] \text { son los }} \\
\text { coeficientes del } \\
\text { polinomio: }\end{array}$ & $\begin{array}{l}\text { si } \quad n_{i}: \text { impar } \Rightarrow \\
\gamma_{i, l+1}^{i}=-\omega_{i}^{u}\left(\xi_{i} \pm \sqrt{1-\xi_{i}^{2}} j\right) \\
l=1,3, \ldots, n_{i}-2 ; \quad \gamma_{n_{i}}^{i}=-\omega_{i}^{o}\end{array}$ & \multirow{2}{*}{$\omega_{i}^{o}$} & $\begin{array}{l}\text { si } \quad n_{i}: \text { impar } \Rightarrow \\
\alpha_{i, l+1}=\operatorname{arctg}\left( \pm \sqrt{1-\xi_{i}^{2}} / \xi\right), \\
l=1,3, \ldots, n_{i}-2 ; \quad \alpha_{n_{i}}=0\end{array}$ \\
\hline & $\begin{array}{l}\prod_{i=1}^{n_{t}}\left(\gamma-\gamma_{l}^{i}\right)=0 \Rightarrow \\
\gamma^{n_{i}}+k_{1 i}^{o} \gamma^{n_{i}-1}+\ldots+k_{n_{i}}^{o}=0\end{array}$ & $\begin{array}{l}\text { si } \quad n_{i}: p a r \Rightarrow \\
\gamma_{i,+1}^{\prime}=-\omega_{i}^{o}\left(\xi_{i} \pm \sqrt{1-\xi_{i}^{2}} j\right) \\
l=1,3, \ldots, n_{i}-1\end{array}$ & & $\begin{array}{l}\text { si } \quad n_{i}: \operatorname{par} \Rightarrow \\
\alpha_{l, n_{+1}}=\operatorname{arctg}\left( \pm \sqrt{1-\xi_{i}^{2}} / \xi\right) \\
l=1,3, \ldots, n_{i}-1\end{array}$ \\
\hline
\end{tabular}




\section{BIBLIOGRAFÍA}

Adebekun, D.K., y Schork, F.J., "Continuos solution polymerization reactor control. 1. Nonlinear reference control of methyl methacrylate polymerization", Ind. Eng. Chem. Res., 28, 1308 (1989a).

Adebekun, D.K., y Schork, F.J., "Continuos solution polymerization reactor control. 1. Nonlinear reference control of methyl methacrylate polymerization", Ind. Eng. Chem. Res., 28, 1308 (1989b).

Alvarez, J., “Output-Feedback Control of Nonlinear Plants”, AIChE J., 42(9), 2540 (1996).

Alvarez, J., "Nonlinear State Estimation with Robust Convergence", J. Proc. Cont., 10, 59 (2000).

Alvarez, J., Hernández, E., y Sánchez, R., "Nonlinear Control of a Continuos Stirred Tank Reactor”, Chem. Eng. Sci., 44 (5), 1147 (1990).

Alvarez, J., y López, T., "Robust dynamic state estimation of nonlinear plants", AIChE J., 45 (1), 107 (1999).

Alvarez, J., López, T., y Hernández, E., "Robust estimation of free-radical homopolymer reactors”, Proc. IFAC Conf. (Beijing, China), 7, 121 (1999).

Alvarez, J., López, T., y Hernández, E., "Robust estimation of free-radical homopolymer reactors”, J. Process Control, 10 (5), 389 (2000).

Alvarez, J., López, T., y Hernández, E., "Robust geometric nonlinear control of process systems”, Proc. IFAC - ADCHEM Symp. (Italia, Pisa), 1, 395 (2000).

Alvarez J., López T., y Hernández, E., "Measurement-driven nonlinear geometric control via estimation-based recovery of state-feedback dynamics", en preparación para enviarse a J. Process Control (2000).

Alvarez, J., Romagnoli, J.A., y Stephanoopoulos, G., "Variable measurement structures for the control of a tubular reactor", Chem. Eng. Sci, 36 (10), 1695 (1981).

Alvarez, J., Suárez, R., y Sánchez, A., "Semiglobal Nonlinear Control based on Complete Input-Output Linearization $\mathrm{y}$ its Application to the Start-up of a Continuous Polymerization reactor”, Chem. Eng. Sci., 49 (21), 3621 (1994).

Arzamendi, G., Leiza, J.R., y Asua, J.M., "Semicontinuous emulsion copolymerization of methy methacylate and ethyl acrylate”, J. Appl. Polym. Sci., 29, 1549 (1991).

Balakotaiah, V., y Luss, D., "Input multiplicity in lumped parameter systems", Chem. Eng. Commun., 39, 309 (1985). 
Bar, D., y Pinto, J.C., "Refractive index of solutions containing poly (vinyl acetate) and poly (methyl methacrylate)”, J. Appl. Polym. Sci., 42, 2795 (1991).

Barrati, R., Alvarez, J., y Morbidelli, M., "Design y experimental Verification of a Nonlinear Catalytic Reactor Estimator", Chem. Eng. Sci., 48, 2573 (1993).

Bequette, B.W., "Nonlinear control of chemical processes: A review", Ind. Eng. Chem. Res., 30, 1391 (1991).

Bestle, D., y Zeitz, M., “Canonical form observer design for nonlinear time-variable systems”, Int. J. Control, 38 (2), 419 (1983).

Byrnes, C., Isidori, A., y Willes, J.C., "Passivity, feedback equivalence, and the global stabilization of minimum phase systems", IEEE Trans. Automatic Control, 36 (11), 1228 (1991).

Boyce, W.E., y DiPrima, R.C., Elementary differential equations and boundary value problems, John Wiley, New York (1991).

Bondy, F., y Lippa, S., "Heat transfer in agitated vessels", Chem. Eng., 4, 62 (1983).

Brandrup, J., e Immergut, E.H., Polymer Handbook, Wiley-Interscience, New York (1989).

Canegallo, S., Storti, G., Morbidelli, M., y Carra, S., "Densimetry for on-line conversion monitoring in emulsion homo- and copolymerization", J. Appl. Polym. Sci., 47, 961 (1993).

Ciccarella, G., Dalla Mora, M., y Germani, A., “A Luenberger-like observer for nonlinear systems”, Int. J. Control, 57 (3), 537 (1993).

Congalidis, J.P, Richards, J.R., y Ray, W.H., “Feedfoward and Feedback Control of Solution Copolymerization Reactor”, AlChE J., 35 (6), 891 (1989).

Congalidis, J.P., y Richards, J.R., "Process control of polymerization reactors: An industrial perspective”, Polym. Reaction Eng., 6(2), 71 (1998).

Crowley, T.J., y Choi, K.Y., "In-line dielectric monitoring of monomer conversion in a batch polymerization reactor", J. Appl. Polym. Sci., 55, 1361 (1995).

Chien, D.C.H., y Penlidis, A., "On-line sensors for polymerization reactors", JMS-Rev. Macromol. Chem. Phys., C30 (1), 1 (1990).

Dautidis, P., y Kravaris, C., "Dynamic output feedback control of minimum-phase nonlinear processes”, Chem. Eng. Sci., 49 (4), 433 (1994).

D'azzo, J.J., y Houpis, C.H., Linear Control System Analysis and Design: Conventional and Modern, McGraw-Hill, New York (1995).

Deza, F., Busvelle, E., Gauthier, J.P., y Rakotopara, D., "High Gain Estimation for Nonlinear Systems", Systems and Control Letters, 18, 295 (1992a). 
Deza, F., Busvelle, E., y Gauthier, J.P., "Exponentially Converging Observers for Distillation Columns and Internal Stability of The Dynamic Output Feedback", Chem. Eng. Sci., 15 (20), 3935 (1992b).

Deza, F., Bossanne, D. , Busvelle, E., Gauthier, J.P., y Rakotopara, D., "Exponential Observers for Nonlinear Systems", IEEE Trans. Automatic Control, 38 (3), 482 (1993).

Dimitratos, J., Georgakis, C., El-Aasser, M., y Klein, A., "Dynamical modeling and state estimation for an emulsion copolymerization reactor", Chem. Eng. Sci., 13, 21 (1989).

Dimitratos, J., Georgakis, C., El-Aasser, M., y Klein, A., "An Experimental Study of Adaptive Kalman Filtering in Emulsion Copolymerization", Chem. Eng. Sci., 46, 3203 (1991).

Dochain D., Perrier, M., y Ydstie, B.E., "Asymptotic Observers for Stirred Tank Reactors", Chem. Eng. Sci., 47, 4167 (1992).

Doyle, F.J., "Nonlinear inferential control for process applications", J. Proc. Cont., 8, 339 (1998).

Elicabe, C.E., y Meira, G.R., "Estimation y control in polymerization reactors", Polymer. Eng. Sci., 28(3), 121 (1988).

Ellis, M.F., Taylor, T.W., Gonzalez, V., y Jensen, K.F., "Estimation of the Molecular Weight Distribution in Batch Polymerization", AIChE J., 34, 1341 (1988).

Ellis, M.F., Taylor, T.W., y Jensen, K.F., "On-line Molecular Weight Distribution Estimation y Control in Batch Polymerization", AIChE J., 40, 445 (1994).

Flory, P.D., Principles of Polymer Chemistry, Cornell UP, New York (1953).

Gauthier, J.P., Hammouri, H., y Othman, S., "A simple observer for nonlinear systems applications to bioreactors", IEEE Trans. Automatic Control, 36 (6), 875 (1992).

Gelb, A., Applied Optimal Estimation, MIT press, Cambridge (1974).

González, R., López, T., y Alvarez, J., "Modelado y operación de reactores de polimerización alquidálicos", Memorias del Congreso Nacional AMIDIQ (2000).

Guckenheimer, J., y Holmes P., Nonlinear oscillations, dynamical systems, and bifurcations of vector fields, Springer-Verlag, New York, (1983).

Hamer, J.W., Akramov, T.A., y Ray, W.H., "The Dynamic Behavior of Continuos Polymerization Reactors - II. Nonisothermal Solution Homopolymerization y Copolymerization in a CSTR", Chem. Eng. Sci. 36, 1897 (1981).

Henderson, L.S., "Stability analysis of polimerization in continuos stirred-tank reactors", Chem. Eng. Prog., 3, 42 (1987).

Henson, M.A., y Seaborg, D.E., “An Internal Model Control Strategy for Nonlinear Systems”, Am. Inst. Chem. Engngs. J., 37(7), 1065 (1991). 
Hernández, H., "Estimación en reactores de homopolimerización con mediciones discreto-retardadas", Tesis de Doctorado en desarrollo, Universidad Autónoma Metropolitana - Iztapalapa, México (2000)

Hernández, H., Hernández E., y Alvarez, J., "Detección de fallas en un reactor de polimerización", Memorias del Congreso Nacional AMIDIQ (2000).

Hirschorn, R.M., "Invertibility of multivariable nonlinear control systems", IEEE Trans. Automatic Control, AC-24 (6), 855 (1979).

Hoogendoorn, K., y Shaw, R., "Control of polymerization processes", Proc. IFAC PRP 4 Automation (Ghent, Belgium), 623 (1980).

Hyun, J.C., Graessley, W.W., y Bankoff, S.G., "Continuous Polymerization of Vinyl Acetate: I. Kinetic Modeling; II. On-Line Estimation of Process Drift", Chem. Eng. Sci., 31, 945 (1976).

Isidori, A., Nonlinear control systems, Springer-Verlag, New York (1995).

Jazwinski, A.H., Stochastic Processes y Filtering Theory, Academic Press, New York (1970).

Jo, J.H., y Bankoff, S.G., "Digital Monitoring y Estimation of Polymerization Reactors", AIChE J., 22, 361-369 (1976).

Johnson, C.D., "Optimization of a certain quality of complete controllability y observability for linear dynamical systems", Trans. ASME, 91, 228 (1969).

Khalil, H.K., Nonlynear Systems, Macmillan Press, New York (1992).

Kalman, R.E., Ho, Y.C., y Narendra, K.F., "Controllability of linear dynamical systems", Contrib. Differential Equations, 1, 189 (1962).

Kantor, J., "A Finite Dimensional Nonlinear Observer for an Exothermic Stirred-Tank Reactor", Chem. Eng. Sci., 44(7), 1503 (1989).

Kontogeorgis, G.M., y Fredeslund, A., "Equations of State and Activity Coefficient Models for Vapor - Liquid Equilibria of Polymer Solutions”, AIChE J., 40, 1711 (1994).

Kou, S.R., Elliot, D.L., y Tarn, T.D., “Observability of Nonlinear Systems”, Information and Control, 22, 89 (1973).

Kozub, D.J., y MacGregor, J.F., "Feedback control of polymer quality in semibatch copolymerizaton reactors", Chem. Eng. Sci., 47 (4), 929 (1992a).

Kozub, D.J., y MacGregor, J.F., "State Estimation for Semi-Batch Polymerization Reactors", Chem. Eng. Sci., 47 (5), 1047 (1992b).

Kravaris, C., y Chung, C., "Nonlinear State-Feedback Synthesis by Global Input/Output Linearization”, AIChE J., 33, 73 (1987).

Kravaris, C., y Soroush, M., "Synthesis of multivariable nonlinear controllers by input/output linearization”, AIChE J., 36 (2), 249 (1990). 
Krener, A., e Isidori, A., "Linearization by Output Injection and Nonlinear Observers", Systems and Control Letters, 3, 47 (1983).

Krener, A., y Respondek, W., "Nonlinear Observers with Linearizable Error Dynamics", SIAM J. Control and Optimization, 23 (2), 197 (1985).

Kumar, S., y Seinfeld, J.H., "Optimal location of measurements in tubular reactors", Chem. Eng. Sci., 33, 1507 (1978).

Kwakernaak, H., y Sivan, R., Linear Optimal Control Systems, John Wiley \& Sons Inc., New York (1972).

Lau, H., Alvarez, J., y Jesen, K.F., "Synthesis of control structures by singular value analysis: Dynamic measures of sensitivity y interaction", AIChE J., 31(3), 427 (1985).

Leiza, J.R., Arzamendi, G., y Asua, J.M., "Copolymer composition control in emulsion polymerization using technical grade monomers", Polymer Int., 30, 455 (1993).

Limqueco, L., Kanto, J.C., y Harvey, S., "Nonlinear adaptive observation of an exothermic stirred-tank reactor”, Chem. Eng. Sci., 46, 797 (1991).

López, T., "Control no lineal con retroalimentación de mediciones en un reactor de copolimerización en solución", Tesis de maestría, Universidad Autónoma Metropolitana - Iztapalapa, México (1995).

López, T., "Problemas de Estimación No Lineal en Reactores Industriales de Polimerización", Primer Taller Nacional de Observación y Estimación de Sistemas No-lineales y sus Aplicaciones (UNAM, México), en prensa (2000).

López T., y Alvarez, J., "Inferencia de estados en un reactor continuo de copolimerización”, Avances en Ing. Quim., 6 (1), 78 (1996).

López T., y Alvarez, J. , "Una metodologia para estimación de estados no lineal en un reactor de copolimerización”, Avances en Ing. Quim., 7 (1), 98 (1997).

López T., y Alvarez, J., "Control de un reactor de copolimerización mediante mediciones en linea”, Memorias del Congreso nacional AMIDIQ, 1, 463 (1998).

López T., y Alvarez, J., "Nonlinear measurement-feedback control of a solution copolymer reactor", Proc. IASTED International Conference - Control and Applications (Hawaii, USA), 1, 194 (1998).

López T., y Alvarez, J. , "Robust nonlinear estimation with the estimability structure as design of freedom", sometido a consideración AIChE J. (2000).

López T., Alvarez, J., y Padilla, S., "Control de la composición en un reactor de copolimerización mediante retroalimentación de mediciones”, Avances en Ing. Quim., 5 (3), 285 (1995). 
López T., Alvarez, J., y Padilla, S., "State estimation of a continuos solution copolymerization reactor", Proc. IFAC - ADCHEM Symp. (Banff, Canada), 1, 213 (1997).

López T., Hernández, H., y Alvarez, J., "Robust nonlinear estimation of alkyd reactors with discrete-delayed measurements", Proc. IFAC - ADCHEM Symp. (Italia, Pisa), 1, 359 (2000).

López-Serrano, F., Fernyez, F., Puig, J.E., y Alvarez, J., “Determination of parameters in 0-1 emulsion polymerization", Proc. Symp. Polymer Disperse Media, Wiley, in press (1999a).

López-Serrano, F., Puig, J.E., y Alvarez, J., "Estimation of parameters in Emulsion and Microemulsion Systems”, Macromol, sometido a consideración (1999b).

Lorimer, J.W., "Refractive index increments of polymers in solution: 1. General theory", Polymer, 13, 46 (1972a).

Lorimer, J.W., "Refractive index increments of polymers in solution: 3. Dependence on concentration", Polymer, 13, 274 (1972b).

Lorimer, J.W., y Jones, D.E.G., "Refractive index increments of polymers in solution: 2. Refractive index increments and light-scattering in polydisperse systems of low molecular weight", Polymer, 13, 52 (1972).

Luenberger, D.G., "Observing the state of a linear system", IEEE Trans. Mil. Electron., MIL-8, 74 (1964).

Luenberger, D.G., "An introduction to observers", IEEE Trans. Automatic Control, AC16 (6), 596 (1971).

Macgregor, J.F., y Ridwell, P.W., "Modeling and control of continuous industrial polymerization reactors”, Computer applications to chemical Eng., ACS Symp. Ser., Squires \& Reklaitis (Eds.), 124 , 2511 (1980).

Middleton, D., An introduction to statistical communication theory, McGraw Hill, New York (1960).

Moore, B.C., "Principal component analysis in linear systems: controllability, observability, y model reduction", IEEE Trans. Automatic Control, AC-26(1), 17 (1981).

Muller, P.C., y Weber, H.I., "Analysis of certain qualities of controllability y observability for linear dynamical systems”, Automatica, 72, 237 (1972).

Mutha, R.K., Cluett, W.R., y Penlidis, A., "A new multirate-measurement-based estimator: emulsion copolymerization batch reactor case study", Ind. Eng. Chem. Res., 36, 1036 (1997a).

Mutha, R.K., Cluett, W.R., y Penlidis, A., "On-line nonlinear model-based estimation and control of a polymer reactor", AIChE J., 43 (11), 3042 (1997b). 
Nijmeijer H., y van der Schaft, A., Nonlinear dynamical control systems, SpringerVerlag, New York (1990)

Ogata, K., Ingeniería de control moderna, Prentice Hall, México-Englewood Cliffs (1980).

Padilla S., Alvarez, J., y López, T., "A nonlinear approach to the problem of composition control in a copolymerization reactor", Proc. American Control Conference (Washington, USA), 5, 3178 (1995).

Padilla, S., Alvarez, J., Alvarez, J.J., y Suarez, R., "Nonlinear analysis of the control problem in a continuous copolymerization reactor," Proc. American Control Conference (1991).

Padilla, S., y Alvarez, J., "Control of Continuous Copolymerization Reactors," AIChE $J ., 43(2), 448$ (1997).

Phani, B.S, y Bequette, W., "Nonlinear Model-Predictive Control: Closed-Loop Stability Analysis", AIChE J., 42(12), 3388 (1996).

Pinto, J.C., y Ray, W.H., "The Dynamic Behavior of Continuos Solution Polymerization Reactors- VII. Experimental Study of Copolymerization Reactor", Chem. Eng. Sci., 50, 715 (1995a).

Pinto, J.C., y Ray, W.H., "The Dynamic Behavior of Continuos Solution Polymerization Reactors- VIII. A Full Bifurcation Analysis of the Lab-scale Copolymerization Reactor", Chem. Eng. Sci., 50, 1041 (1995b).

Ponnuswamy, S., y Shah, S.L., "On-Line Monitoring of Polymer Quality in a Batch Polymerization Reactor”, J. Appl. Polym. Sci., 32, 3239 (1986).

Ponnuswamy, S., Shah, S.L., y Kiparissides, C.A., "Computer optimal control of batch polymerization reactors”, Ind. Eng. Chem. Res., 26, 2229 (1987).

Ray, W.H., "Molecular Weight Distributions in Copolymer Systems. I. Living Copolymers", Macromolecules, 4 (2), 162 (1971).

Ray, W.H., "On the mathematical modeling of Polymerization Reactors", J. Macromol. Sci.-Revs. Macrolmol. Chem., C8 (1), 1 (1972).

Ray, W.H., “Polymerization Reactor Control”, IEEE Control systems, 6 (4), 3 (1986).

Ray, W.H., Douglas, T.L., y Godsalve E.W., "Molecular Weight Distributions in Copolymer Systems. II. Free Radical Copolymerization”, Macromolecules, 4 (2), 166 (1971).

Reid, R.C., Praunitz, J.M., y Poling, B.E., The properties of gases and liquids, McGraw-Hill, New York (1987).

Romagnoli, J.A., Alvarez, J., y Stephanoopoulos, G., "Variable measurement structures for process control”, Int. J. Control, 33(2), 269 (1981). 
Schmidt, A.D., Clinch, A.B., y Ray, W.H., "The dynamic behavior of continuos polymerization reactors-III. An experimental study of multiple steady states in solution polymerization", Chem. Eng. Sci., 39 (3), 419 (1984).

Schuler, H., "Estimation of states in a Polymerization Reactor", Proc. IFAC PRP 4 Automation (Ghent, Belgium), 1, 369 (1980).

Schuler, H., y Papadopoulou, S., "Real-Time Estimation of the Chain Length Distribution in a Polymerization Reactor-II. Comparison of Estimated and Measured Distribution Functions", Chem. Eng. Sci., 41 (10), 2681 (1986).

Schuler, H., y Sushen, Z., "Real-Time Estimation of the Chain Length Distribution in a Polymerization Reactor", Chem. Eng. Sci., 40, 1891 (1985)

Schork, F.J., "Sensors and controls for emulsion polymerization reactors", Advances in Emulsion Polym. and Latex Technol., 1, (1991)

Shannon, C.E., "Mathematical theory of communication", Belt System Technical J., 27, 379,623 (1948).

Sharma, D.K., y Soane, D.S., "High-conversion diffusion-controlled copolymerization kinetics", Macromol., 21(3), 700 (1988).

Silverman, L.M., y Meadows, H.E., "Controllability and observability in time-variable linear systems”, J. SIAM Control, 5 (1), 64 (1967).

Slotine, J.J., y Li, W., Applied Nonlinear Control, Prentice-Hall, Englewood Cliffs, New Jersey (1991).

Slotine, J.J.E., Hedrick, J.K., y Misawa, E.A., "On Sliding Observers for Nonlinear Systems", J. of Dyn. Systems, Meas. and Contr., 109, 245 (1987).

Soroush, M., y Kravaris, C., "Nonlinear Control of a Batch Polymerization Reactor: An Experimental Study", AIChE J., 38 (9), 1429 (1992).

Soroush, M., y Kravaris, C., "Multivariable Nonlinear Control of a Continuous Polymerization Reactor: An Experimental Study", AIChE J., 39(12), 1920 (1993).

Soroush, M., y Kravaris, C., "Nonlinear Control of a Polymerization CSTR with Singular Characteristic Matrix", AIChE J., 40 (6), 980 (1994).

Soroush, M., "Nonlinear State-Observer Design with Application to Reactors", Chem. Engng. Sci., 52(5), 387 (1997).

Stephanopoulos, G., Chemical Process Control, Prentice Hall, Englewood Cliffs, New Jersey (1984).

Sussmann, H.J., y Kokotovic, P.V., "The peaking phenomenon y the global stabilization of nonlinear systems", IEEE Trans. Automatic Control, 36(4), 4241 (1991).

Tatirajau, S., y Soroush, M., "Nonlinear State Estimation in a Polymerization Reactor", Ind. Eng. Chem. Res., 36, 2679 (1997). 
Teymour, F., y Ray, W.H., "The dynamic behavior of continuos polymerization reactors-IV. Dynamic stability and bifurcation analysis of an experimental reactor", Chem. Eng. Sci., 44 (9), 1967 (1989).

Teymour, F., y Ray, W.H., "The dynamic behavior of continuos polymerization reactors-V. Experimental investigation of limit-cycle behavior for vinyl acetate polymerization", Chem. Eng. Sci., 47 (15/16), 4121 (1992).

Teymour, F., y Ray, W.H., "The dynamic behavior of continuos polymerization reactors-VI. Complex dynamics in full-scale reactors", Chem. Eng. Sci., 47 (15/16), 4133 (1992).

Tsinias, J., "Observer Design for Nonlinear Systems", Systems and Control Letters, 13, 135 (1989).

Tsinias, J., "Further Results on the Observer Design Problem", Systems and Control Letters, 20, 411 (1990).

Valluri, S., y Soroush, M., "Nonlinear state estimation in the presence of multiple steady states", Ind. Chem. Eng. Res., 35, 2645 (1996).

Van Dooting, M., Rakotrapa, V.D., Gauthier, J.P., y Hobbes, P., "Nonlinear Deterministic Observer for State Estimation: Application to a Continuous FreeRadical Polymerization Reactor", Comput. Chem. Eng., 16, 777 (1992).

Van Doremaele, G.H.J., "Model Prediction, Experimental Determination, and Control of Emulsion Copolymer Microstructure", PhD Thesis, Eindhoven Technische Universiteit, Eindhoven, The Netherlys (1990).

Vidyasagar, M., Nonlinear Systems Analysis, Prentice-Hall, New York (1978).

Walcott, B.L., Corless, M.J., y Zak, S.H., "Comparative Study of Nonlinear StateObservation Techniques," Int. J. Control, 45 (6), 2109 (1987).

Waldraff, W., Dochain, D., Bourrel, S., y Magnus, A., "on the use of observability measures for sensor location in tubular reactors", J. Proc. Contr., 8 (5-6), 497 (1998).

Wang, G.B., Peng, S.S., y Haung, H.P., "A Sliding Observer for Nonlinear Process Control”, Chem. Eng. Sci., 52(5), 787 (1997).

Wonham, W.M., Linear Multivariable Control. A Geometric Approach, Springer-Verlag, New York (1985).

Xia, X., y Gao, W., "Nonlinear Observer Design by Observer Error Linearization", SIAM J. on Contr. and Optim., 27, 199 (1989). 